\title{
MRI of Heterogeneous Hydrogenation Reactions Using Parahydrogen Polarization
}

\author{
by \\ Scott Russell Burt \\ Department of Chemistry \\ University of California, Berkeley \\ and \\ Materials Sciences Division \\ Lawrence Berkeley National Laboratory \\ Berkeley, CA 94720
}

Committee in charge:

Professor Alexander Pines, Chair

Professor David E. Wemmer

Professor John Clarke

Fall 2008 


\section{MRI of Heterogeneous Hydrogenation Reactions Using Parahydrogen Polarization}

Copyright Fall 2008

by

Scott Russell Burt

This document was prepared as an account of work sponsored by the United States Government. While this document is believed to contain correct information, neither the United States Government nor any agency thereof, nor The Regents of the University of California, nor any of their employees, makes any warranty, express or implied, or assumes any legal responsibility for the accuracy, completeness, or usefulness of any information, apparatus, product, or process disclosed, or represents that its use would not infringe privately owned rights. Reference herein to any specific commercial product, process, or service by its trade name, trademark, manufacturer, or otherwise, does not necessarily constitute or imply its endorsement, recommendation, or favoring by the United States Government or any agency thereof, or The Regents of the University of California. The views and opinions of authors expressed herein do not necessarily state or reflect those of the United States Government or any agency thereof or The Regents of the University of California. 


\begin{abstract} by

Scott Russell Burt

Doctor of Philosophy in Chemistry

University of California at Berkeley

Professor Alexander Pines, Chair
\end{abstract}

MRI of Heterogeneous Hydrogenation Reactions Using Parahydrogen Polarization

The power of magnetic resonance imaging (MRI) is its ability to image the internal structure of optically opaque samples and provide detailed maps of a variety of important parameters, such as density, diffusion, velocity and temperature. However, one of the fundamental limitations of this technique is its inherent low sensitivity. For example, the low signal to noise ratio (SNR) is particularly problematic for imaging gases in porous materials due to the low density of the gas and the large volume occluded by the porous material. This is unfortunate, as many industrially relevant chemical reactions take place at gas-surface interfaces in porous media, such as packed catalyst beds. Because of this severe SNR problem, many techniques have been developed to directly increase the signal strength. These techniques work by manipulating the nuclear spin populations to produce polarized (i.e. non-equilibrium) states with resulting signal strengths that are orders of magnitude larger than those available at thermal equilibrium. 
This dissertation is concerned with an extension of a polarization technique based on the properties of parahydrogen. Specifically, I report on the novel use of heterogeneous catalysis to produce parahydrogen induced polarization and applications of this new technique to gas phase MRI and the characterization of micro-reactors. First, I provide an overview of nuclear magnetic resonance (NMR) and how parahydrogen is used to improve the SNR of the NMR signal. I then present experimental results demonstrating that it is possible to use heterogeneous catalysis to produce parahydrogen-induced polarization. These results are extended to imaging void spaces using a parahydrogen polarized gas. In the second half of this dissertation, I demonstrate the use of parahydrogen-polarized gasphase MRI for characterizing catalytic microreactors. Specifically, I show how the improved SNR allows one to map parameters important for characterizing the heat and mass transport in a heterogeneous catalyst bed. This is followed by appendices containing detailed information regarding the design and use of my experimental setup.

Professor Alexander Pines Dissertation Committee Chair 


\section{To Judy Seydel}

My love for chemistry began in your class;

thank you for starting me on this path. 


\section{Acknowledgements}

First and foremost, I must express a debt of gratitude to my wife, Stephanie. Her patience and support were key to my success. I would particularly like to acknowledge her excellent editorial skills and many critiques that have benefitted not only this document, but many of my other presentations. I must also thank my children, Sarah and Peter, whose curiosity and energy have been a reminder of the excitement found in exploration and learning.

My thanks and gratitude go out to my collaborators and co-workers, without whom this work could not have been done. My thanks to Igor Koptyug and Song-I Han, for initiating this project and creating the fruitful collaboration between the International Tomography Center in Novosibirsk and Alex's lab. Thanks to Christian Hilty for helping me build the first parahydrogen setup and getting me started in the lab. A special thanks to Kirill Kovtunov; his expertise with catalysts and his many creative ideas were invaluable to the success of this work. I am particularly indebted to Sabieh Anwar, whose excitement and dedication provided the support and motivation to get me through a difficult and frustrating period of my research. Many, many thanks to Louis Bouchard. I consider myself extremely lucky to have had Louis as a mentor. From his summer lectures on NMR to our discussions of average Hamiltonian theory to the crash course in MRI during our all-night experiments last summer, Louis has shaped my vision of what NMR is and how to approach interesting problems. My thanks also to David Trease and Thomas Theis, for all their help, discussions, questions and listening ears. And of course, my thanks to Alex. His unconditional and enthusiastic support of his students is truly amazing. The congenial 
atmosphere he cultivates in his lab and his unrestrained excitement and optimism is what brought me to his group. I consider myself lucky to be numbered among the pinenuts and want to thank all the other pinenuts, past and present, who made my time in Alex's lab so enjoyable.

I owe a debt of gratitude to the many professors, teachers and mentors I have had over the years. They have encouraged my interest in learning and problem solving, and have shaped the path my life has followed. I consider myself extremely lucky to have been surrounded by such great examples. In particular, I would like to thank Randy Shirts, for getting me started in research and introducing me to the beauty of statistical mechanics. That I even contemplated a graduate degree is thanks to his support and encouragement. My thanks to Tom Slezak for the summer I had at Lawrence Livermore National Lab. My time in his lab taught me a great deal about myself and how to approach unfamiliar problems. Of the many great teachers and professors I have had, I would like to specifically thank Hitoshi Murayama, Steve Conolly, Matt Asplund, Judy Seydel, Ron Hampton and Mark Hatch; I have many fond memories from their classrooms and the 'aha' moments I experienced therein.

Finally, I want to thank my family. Their support, encouragement and prayers have given me strength. In particular, my love of learning and the life shaping experiences I had in school were largely the result of my parents' high expectations and my constant efforts to imitate my older sister, Lisa. I can vividly remember Lisa explaining to me how fascinating she found Kramer's rule or the rules for naming simple organic compounds. Her excitement in learning new things and her desire to share them with me sparked my 
interest in learning; a flame that has not been extinguished. Furthermore, all of my siblings have devoted an enormous amount of time and effort to mastering skills in art, music and sports. While I have never been particularly good at such activities, my siblings' examples of dedication in mastering these skills and achieving difficult goals has driven me to succeed in my own hobbies and interests. I am further indebted to my parents and grandparents for raising me in an environment that values the personal search for knowledge and truth. Growing up with the perspective that it is my duty to discover for myself what is true, be it spiritual or physical truths, has made all the difference.

The glory of God is intelligence, or, in other words, light and truth.

-Doctrine \& Covenants, 93:36

This research was performed under an appointment to the Department of Homeland Security (DHS) Scholarship and Fellowship Program, administered by the Oak Ridge Institute for Science and Education (ORISE) through an interagency agreement between the U.S. Department of Energy (DOE) and DHS. ORISE is managed by Oak Ridge Associated Universities (ORAU) under DOE contract number DE-AC05-06OR23100. All opinions expressed in this paper are the author's and do not necessarily reflect the policies and views of DHS, DOE, or ORAU/ORISE. This work was supported by the Director, Office of Science, Office of Basic Energy Sciences, Materials Sciences and Engineering Division, of the U.S. Department of Energy (DOE) under contract no. DE-AC02-05CH11231 and the CRDF (grant RU-C1-2581-NO-04). 


\section{Contents}

List of Figures viii

List of Tables $\quad$ x

Symbols and Abbreviations $\quad$ xi

1 Parahydrogen and Sensitivity Enhancement 1

1.1 Introduction to Nuclear Magnetic Resonance . . . . . . . . . . . . . . . 1

1.1.1 Applications of NMR . . . . . . . . . . . . . . 2

1.1.2 Basic Principles. . . . . . . . . . . . . . . 5

1.1.3 Sensitivity and Nuclear Spin Polarization . . . . . . . . . . 11

1.2 Ortho- and Parahydrogen . . . . . . . . . . . . . . . 17

1.2.1 Consequences of Symmetry _. . . . . . . . . . . . . . . 17

1.2.2 Manipulating the Fraction of Parahydrogen . . . . . . . . . . . . . 20

1.3 Parahydrogen-induced Polarization . . . . . . . . . . . . . . . 23

1.3.1 Creating Observable Net Magnetization . . . . . . . . . . . . . 24

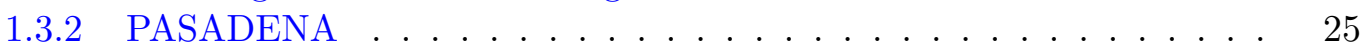

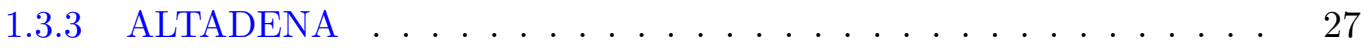

1.3.4 Sensitivity Enhancement . . . . . . . . . . . . . . . 30

1.4 Applications . . . . . . . . . . . . . . . . . . 32

2 Parahydrogen Polarization using Heterogeneous Hydrogenation 34 Koptyug, I. V.; Kovtunov, K. V.; Burt, S. R.; Anwar, M. S.; Hilty, C.; Han, S.-I.; Pines, A.; Sagdeev, R. Z.; Parahydrogen-Induced Polarization in Heterogeneous Hydrogenation Reactions. J. Am. Chem. Soc., 129, (2007) 5580-5586.

2.1 Introduction . . . . . . . . . . . . . . . . . . . 34

2.2 Experimental Methods . . . . . . . . . . . . . . . . . . 37

2.2.1 Supported Catalysts . . . . . . . . . . . . . . 37

2.2.2 Parahydrogen Production . . . . . . . . . . . . . . . . . . . . 38

2.2 .3 Experimental Setup . . . . . . . . . . . . . . . . . . . 38

2.2.4 Heterogeneous Hydrogenation in Solution . . . . . . . . . . . . . 40

2.2.5 Heterogeneous Hydrogenation in Gas Phase . . . . . . . . . . . . . . 40 
2.3 Results. . . . . . . . . . . . . . . . . . . . . . 41

2.3.1 Heterogeneous Hydrogenation in Solution . . . . . . . . . . . . 41

2.3.2 Heterogeneous Hydrogenation in Gas Phase . . . . . . . . . . . . . . 42

2.4 Discussion . . . . . . . . . . . . . . . . . . 46

3 Gas-Phase MRI using Heterogeneous Parahydrogen Polarization $\quad 51$ Bouchard, L.-S.; Kovtunov, K. V.; Burt, S. R; Anwar, M. S.; Koptyug, I. V.; Sagdeev, R. Z.; Pines, A.; Parahydrogen-Enhanced Hyperpolarized Gas-Phase Magnetic Resonance Imaging. Angew. Chem. Int. Ed., 46, (2007) 4064-4068.

3.1 Magnetic Resonance Imaging . . . . . . . . . . . . . . . . . . . . . 51

3.1 .1 Basic Principles . . . . . . . . . . . . . . . . . 51

3.1 .2 MRI of Gases . . . . . . . . . . . . . . . . . . . . 55

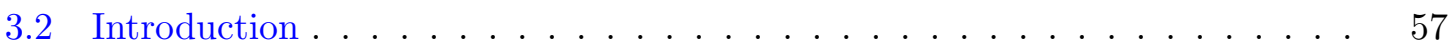

3.3 Experimental Methods . . . . . . . . . . . . . . . . . . . 60

3.4 Results . . . . . . . . . . . . . . . . . . . . . 62

3.5 Discussion . . . . . . . . . . . . . . . . . . 64

4 Micro-reactor Characterization using Heterogeneous PHIP 69

Bouchard, L.-S.; Burt, S. R.; Anwar, M. S.; Kovtunov, K. V.; Koptyug, I. V.; Pines, A.; NMR Imaging of Catalytic Hydrogenation in Microreactors with the Use of para-Hydrogen. Science, 319, (2008) 442-445.

4.1 Introduction . . . . . . . . . . . . . . . . . . 69

4.1.1 Packed Bed Catalytic Micro-reactors . . . . . . . . . . . . . . 69

4.1.2 Methods to Characterize Micro-reactors . . . . . . . . . . . . . . 71

4.1.3 Imaging Parahydrogen Induced Polarization . . . . . . . . . . . . 72

4.2 Results. . . . . . . . . . . . . . . . . . . 75

4.2.1 Imaging Active Regions in the Catalyst Bed . . . . . . . . . . . . 75

4.2.2 Controlled Transport of Polarization within the Catalyst Bed . . . . 75

4.3 Future Applications . . . . . . . . . . . . . . . . . . . . 79

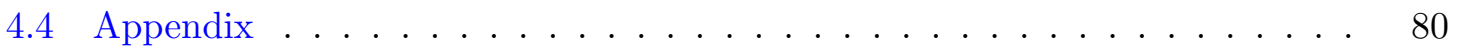

4.4 .1 Equipment . . . . . . . . . . . . . . . . . 80

4.4 .2 Parahydrogen Production . . . . . . . . . . . . . . . . . . 80

4.4.3 Supported Catalyst and Reactor . . . . . . . . . . . . . . 81

4.4 .4 Imaging Pulse Sequence . . . . . . . . . . . . . . . . . . . . 81

4.4.5 Parahydrogen Induced Polarization and Singlet Lifetime . . . . . . . . 83

4.4 .6 Pulsed Field Gradients . . . . . . . . . . . . . . . . . . . . . 84

4.4 .7 Enhancement Factor . . . . . . . . . . . . . . . . 86

5 Mapping Temperature Distribution in a Catalyst Bed $\quad 88$

Burt, S. R.; Bouchard, L.-S.; Theis, T.; Pines, A. Manuscript in Preparation, (2008).

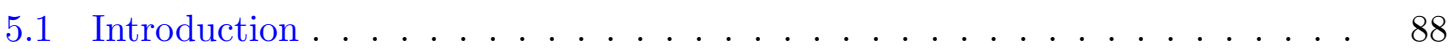


5.1.1 Characterizing Packed Bed Catalytic Micro-reactors . . . . . . . . . 89

5.1 .2 Temperature-Dependent NMR Parameters . . . . . . . . . . . . 90

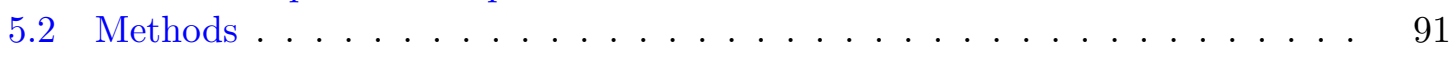

5.3 Results and Discussion . . . . . . . . . . . . . . . 96

6 Microscopic Pressure Maps in a Catalyst Bed 101

Bouchard, L.-S.; Burt, S. R.; Pines, A. Manuscript in Preparation, (2008).

6.1 Introduction . . . . . . . . . . . . . . . . . . . 101

6.2 Methods . . . . . . . . . . . . . . . . . . . . . . . . . . . . . . . . . . . 102

6.2 .1 Navier-Stokes Equation . . . . . . . . . . . . . . . . . . . 102

6.2.2 Transformation to the Poisson Equation . . . . . . . . . . . . . 103

6.2 .3 Numerical Techniques . . . . . . . . . . . . . . . . . . . 105

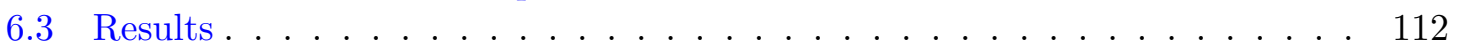

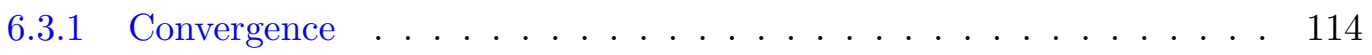

6.3 .2 Initial Conditions . . . . . . . . . . . . . . . . . . 115

6.4 Discussion . . . . . . . . . . . . . . . . . . . . 117

$\begin{array}{ll}\text { A Experimental Setup } & 119\end{array}$

A.1 Parahydrogen Production . . . . . . . . . . . . . . . . . 119

A.1.1 Liquid $\mathrm{N}_{2}$ Converter . . . . . . . . . . . . . . 120

A.1.2 Compressed He Converter . . . . . . . . . . . . . . . . . 123

A.1.3 Testing the Conversion of Ortho- to Parahydrogen . . . . . . . . . . 138

A.2 Gas Flow Setup . . . . . . . . . . . . . . . . . . . 141

A.2.1 Gas Manifold . . . . . . . . . . . . . . . . . . . 142

A.2.2 Flow Control . . . . . . . . . . . . . . . . . . . . . . 144

A.2.3 Safety Controls . . . . . . . . . . . . . . . . . 148

$\begin{array}{ll}\text { B Source Code } & 151\end{array}$

B.1 Pulse Programs . . . . . . . . . . . . . . . . . . . . . . . 151

B.1.1 Calibrating $p-\mathrm{H}_{2}$ Fraction . . . . . . . . . . . . . 151

B.1.2 Bubbling Setup . . . . . . . . . . . . . . . . 153

B.1.3 $p-\mathrm{H}_{2}$ Polarized Void Space Imaging . . . . . . . . . . . . . . 156

B.1.4 Motion Compensated Pulse Sequences for Micro-reactor Imaging . . 158

B.2 Selected Matlab Code . . . . . . . . . . . . . . . . . . . . . 164

B.2.1 Loading Spectrometer Data . . . . . . . . . . . . . . . . . . 164

B.2.2 Model Propane Spectrum for Temperature Mapping . . . . . . . . . 166

B.2.3 Iterative Approach to Poisson Equation . . . . . . . . . . . . . 168

$\begin{array}{ll}\text { Bibliography } & 174\end{array}$ 


\section{List of Figures}

1.1 The Zeeman Effect . . . . . . . . . . . . . . . . . . 7

1.2 Angular Momentum and Precession . . . . . . . . . . . . . 8

1.3 Transverse and Longitudinal Relaxation . . . . . . . . . . . . . . . . . . . . 9

1.4 Nuclear Spin Polarization vs. $B_{0}$ or $T \ldots \ldots \ldots \ldots$

$1.5 \mathrm{H}_{2}$ Rotational Energy Levels . . . . . . . . . . . . . . . . . . 21

1.6 Nuclear Spin States, Direct Product and Symmetric Basis . . . . . . . . . 22

$1.7 \% p-\mathrm{H}_{2}$ vs. Temperature . . . . . . . . . . . . . . . 23

1.8 Molecular Addition of $\mathrm{H}_{2}$ Across a Double Bond . . . . . . . . . . . . . 25

1.9 PASADENA: Sudden Approximation and the Expected Spectrum . . . . . 26

1.10 ALTADENA: Adiabatic Transport and the Expected Spectrum . . . . . . . 29

1.11 Sensitivity Enhancement vs. Fraction of $p-\mathrm{H}_{2} \ldots \ldots \ldots \ldots$

2.1 Schematic of the Experimental Setup . . . . . . . . . . . . . . . . 39

2.2 PASADENA Spectra of Styrene in Solution using 2 Different Heterogeneous Catalysts . . . . . . . . . . . . . . . . . . 43

2.3 ALTADENA Spectrum of Styrene in Solution using a Heterogeneous Catalyst 44

$2.4{ }^{31} \mathrm{P}$ Spectrum of Supernatant after Reaction . . . . . . . . . . . . . . . . . . . 45

2.5 PASADENA Spectrum of Propane Gas . . . . . . . . . . . . . 47

$3.1 \mathrm{k}$-Space Trajectories and Image Reconstruction . . . . . . . . . . . . . . . . 54

3.2 Schematic of Reactions . . . . . . . . . . . . . . . . . 58

3.3 Schematic of the Experimental Setup . . . . . . . . . . . . . . 61

3.4 Thermal and Polarized Spectra of Propylene and Propane . . . . . . . . . 65

3.5 ALTADENA Polarized Images of a Void Space . . . . . . . . . . . . 66

4.1 Schematic of the Micro-reactors . . . . . . . . . . . . . . . . 74

4.2 MR Images of a Heterogeneous Micro-reactor . . . . . . . . . . . . . 76

4.3 Controlled Transport of the Polarized Product _ . . . . . . . . . . . 77

4.4 Imaging Heterogeneous Distributions of Catalyst . . . . . . . . . . . . 79

4.5 Flow-Encoded MRI Pulse Sequence . . . . . . . . . . . . . . . . . 82

$4.6{ }^{1} \mathrm{H}$ Spectra in the Micro-reactor . . . . . . . . . . . . . . . . 87 
5.1 Experimental and Simulated Propane Spectra . . . . . . . . . . . . . . 94

5.2 Image Thresholding . . . . . . . . . . . . . . . . . . . . . . 95

5.3 line width vs. Temperature Calibration . . . . . . . . . . . . . . . 97

5.4 Spatial Dependence of Spectrum Parameters . . . . . . . . . . . . . . . . . . 98

5.5 Temperature Variation within the Catalyst Bed . . . . . . . . . . . . . 99

6.1 Schematic of the Catalyst Bed . . . . . . . . . . . . . . . . 104

6.2 Schematic of $\nabla^{2}$ using Numerical Derivatives . . . . . . . . . . . . 106

6.3 Thresholding the Velocity Phase Map . . . . . . . . . . . . . . . . . 107

6.4 Boundary Effect of Numerical Derivatives . . . . . . . . . . . . . . . . 108

6.5 Microscopic Pressure Map . . . . . . . . . . . . . . . . . . . . . . . 113

6.6 Micro-reactor Flow Setup . . . . . . . . . . . . . . . . . . . . 114

6.7 Rates of Convergence . . . . . . . . . . . . . . . . . . . 115

6.8 Stability with Respect to Initial Conditions . . . . . . . . . . . . . . 116

A.1 Modified Liquid $\mathrm{N}_{2}$ Cryostat for $p-\mathrm{H}_{2}$ Production . . . . . . . . . . . . 121

A.2 Pressure Dependence of $p-\mathrm{H}_{2}$ Fraction . . . . . . . . . . . . . . 122

A.3 Compressed He Cryostat for $p-\mathrm{H}_{2}$ Production . . . . . . . . . . . . . . 124

A.4 Conversion Cell 1: Baffle Insert Schematic . . . . . . . . . . . . . . . 126

A.5 Conversion Cell 1: Sheath Schematic . . . . . . . . . . . . . . . . 127

A.6 Conversion Cell 1: Adapter Plate Schematic . . . . . . . . . . . . . . 128

A.7 Conversion Cell 1: Overall Schematic . . . . . . . . . . . . . . . . 129

A.8 Conversion Cell 1: Machining the Insert, Lathe . . . . . . . . . . . . 130

A.9 Conversion Cell 1: Machining the Insert, Mill . . . . . . . . . . . . 131

A.10 Conversion Cell 1: Machining the Sheath . . . . . . . . . . . . . . 132

A.11 Conversion Cell 1: Machining the Adapter . . . . . . . . . . . . . . . . 133

A.12 Conversion Cell 1: Completed Baffle Design . . . . . . . . . . . . . . . 134

A.13 Conversion Cell 2: Loop Design . . . . . . . . . . . . . . . . . 135

A.14 Conversion Cell 3: Heat Exchange Coil . . . . . . . . . . . . . . . . . 137

A.15 Conversion Cell $1 \& 2$ Mounted on the Cold Finger . . . . . . . . . . . . . . 137

A.16 $o-\mathrm{H}_{2}$ Spectra on Alumina . . . . . . . . . . . . . . . . . . 139

A.17 Calibrating the $p-\mathrm{H}_{2}$ Fraction . . . . . . . . . . . . . . . . 140

A.18 Schematic of the Initial Flow Design . . . . . . . . . . . . . . . . . . . 142

A.19 Schematic of Second Flow Design . . . . . . . . . . . . . . . . 143

A.20 Schematic of Final Flow Design . . . . . . . . . . . . . . . . . 145

A.21 $p-\mathrm{H}_{2}$ Flow Setup . . . . . . . . . . . . . . . 146

A.22 Control Panel and Monitoring Devices . . . . . . . . . . . . . . . . 148

A.23 Circuit Diagram for Flow Control . . . . . . . . . . . . . . . . . 149

B.1 Spin Echo Acquisition of $o-\mathrm{H}_{2}$ Signal on alumina . . . . . . . . . . 151

B.2 TTL Controlled Bubbling Sequence . . . . . . . . . . . . . . . . . 154

B.3 Void Space Imaging using ALTADENA Polarization . . . . . . . . . . . . . 156

B.4 PASADENA Imaging in a Reactor Bed . . . . . . . . . . . . . . 158 


\section{List of Tables}

1.1 Hamiltonians for Liquid State NMR . . . . . . . . . . . . . . . . . 11

1.2 Symmetry Coupling of Rotational and Nuclear Spin States . . . . . . . . . 19

A.1 Calibration of Liquid $\mathrm{N}_{2}$ Conversion Cell . . . . . . . . . . . . . . . . 141 


\section{Symbols and Abbreviations}

\section{Variables}

$\vec{B} \quad$ magnetic field vector

$B_{0} \quad$ amplitude of the static magnetic field

$B_{1} \quad$ amplitude of the transverse magnetic field, $B_{1} \mathrm{e}^{-i \omega_{r f} t}$

$\vec{B}_{\text {eff }}$ effective magnetic field vector in the rotating frame, $\vec{B}_{\text {eff }}=\left(B_{0}-\frac{\omega_{r f}}{\gamma}\right) \hat{z}+\vec{B}_{1}$

$\mathcal{H} \quad$ Hamiltonian or total energy operator of a system

I spin operators

$J_{i j} \quad$ isotropic $J$ coupling

$M_{0} \quad$ net magnetization

$t \quad$ evolution time in the direct dimension of $2 \mathrm{D}$ experiment

$T_{1} \quad$ spin-lattice relaxation rate constant

$T_{2} \quad$ spin-spin relaxation rate constant

$\tau \quad$ evolution time in the indirect dimension of $2 \mathrm{D}$ experiment

$\omega \quad$ Larmor precession frequency, $\omega=\gamma|B|$, in $\mathrm{rad} / \mathrm{sec}$

$\omega_{\text {rf }} \quad R F$ (Radio Frequency) field strength in $\mathrm{rad} / \mathrm{sec}$ 


\title{
Physical Constants
}

\author{
$h \quad$ Planck's constant (quantum of action), $6.626176 \times 10^{-34} \mathrm{Js}$ \\ $\hbar \quad \hbar=h / 2 \pi$ \\ $k \quad$ Boltzmann constant, $1.380662 \times 10^{-23} \mathrm{JK}^{-1}$ \\ $R \quad$ Molar gas constant, $8.314510 \mathrm{~J}(\mathrm{~mol} \mathrm{~K})^{-1}$ \\ $\gamma \quad$ gyromagnetic ratio
}

\section{Mathematical Operations}

$\mathcal{F}\{\} \quad$ Fourier Transform, $\mathcal{F}\{g(\vec{x})\}=\frac{1}{\sqrt{2 \pi}} \int \mathrm{e}^{-l \vec{k} \cdot \vec{x}} g(\vec{x}) \mathrm{d} \vec{x}$

$\otimes \quad$ Convolution, $(f \otimes g)(t)=\int f(\tau) g(\tau-t) \mathrm{d} \tau$ 


\section{Chapter 1}

\section{Parahydrogen and Sensitivity}

\section{Enhancement}

\subsection{Introduction to Nuclear Magnetic Resonance}

A comprehensive introduction to the experimental and theoretical techniques employed by the various fields of nuclear magnetic resonance (NMR) would fill the pages of several books. Thus, the following introduction is intended only as a brief review to introduce the scope of NMR techniques (sec. 1.1.1), the basic concepts and terminology used in NMR (sec. 1.1.2), and the problem of low sensitivity in NMR (sec. 1.1.3). The reader is encouraged to use the references provided to further explore the details of these fascinating techniques. 


\subsubsection{Applications of NMR}

Nuclear magnetic resonance is a technique that probes the local molecular environment via application and detection of radio-frequency $(\mathrm{RF})$ signals. It has been over 60 years since the first observations of NMR by Bloch and Purcell $[30,128]$ and the phenomenon of magnetic resonance has laid the foundation for a wide variety of sophisticated techniques that find important and routine use in a diverse number of fields.

For example, NMR plays a pivotal role in chemistry [51] where it is used for chemical identification [126], structure determination [48,63,119], and characterization of molecular dynamics $[101,119]$. NMR has also become an essential tool to the medical community in the guise of magnetic resonance imaging (MRI) [121]. Within the field of chemical engineering, NMR provides methods to measure and visualize flow distributions, diffusion and other forms of molecular dynamics $[43,89,142]$, as well as providing techniques for studying catalysis and reaction kinetics [142]. Solid state NMR [101,138] and NMR

microscopy $[32,43]$ provide important tools for characterizing molecular structure and dynamics of crystalline, polycrystalline, porous, and soft materials. Even fields such as oil exploration [146] and food science [22] employ tools based on the principles of NMR.

There are a number of advantages that make NMR-based techniques appealing: the interior of optically opaque media can be probed non-invasively and without the use of ionizing radiation, sensitivity to a large variety of molecular parameters, and the large degree of control over the quantum evolution of the system. Because the manipulation and detection of signals occurs via magnetic induction, optical opacity is no hindrance. This allows the interior of opaque or delicate samples to be characterized without physically al- 
tering or damaging the sample $[43,121]$. This is, perhaps, most evident in medical imaging, where one can avoid exposing the patient to ionizing radiation or invasive surgery by using MRI to image the interior of the body. Non-invasive characterization is also important when the dynamics or processes of interest would be altered by the presence of external probes, such as transducers or tracers. For example, NMR allows the measurement of self-diffusion and flow profiles without the addition of tracer particles [43]. In microscopic volumes, flow and other parameters can be measured where the presence of a probe would disturb the system due to its large size relative to the size of the micro-channel [34]. With the growing importance of micro-fluidic lab-on-a-chip technology, MRI is emerging as a powerful tool because of this ability to monitor chemical reactions, kinetics and flow on small length scales [142]. Industrial applications, such as quality or process control, also benefit from non-invasive imaging where money is saved by not damaging or consuming a product during characterization $[22,142]$. The spectroscopic appeal of NMR lies in its sensitivity to molecular structure, environment, and dynamics [48]. Similarly, medical and materials imaging employ NMR's sensitivity to these parameters to provide image contrast for visualizing specific parameters of interest, such as temperature, velocity, strain or diffusion [32]. Finally, the great variety of NMR techniques possible is due largely to the concept of selective averaging that allows the experimentalist to have a large degree of control over the information content provided by the NMR signal [101,138].

Despite the advantages and utility of these techniques, their routine use and broader application in the field is fundamentally limited by the inherent poor sensitivity of NMR [51] (sec. 1.1.3). Typically, a tradeoff must be made between time, resolution, 
and sensitivity. In situations where sensitivity or resolution can be sacrificed or where long scan times are not problematic, NMR techniques have become mainstays. However, there are many applications where the sensitivity is already poor and either high resolution or a reasonable scan time is a must. An example of the first case is micro-imaging, in which case high resolution is a requirement. The standard approach would require inordinate amounts of signal averaging. However, signal averaging time grows quadratically and quickly becomes infeasible. Medical imaging is a good example of the second case, where scan times must be short for a variety of reasons [121]. Again, the standard approach requires the sacrifice of resolution, which is often unacceptable, e.g. with diagnostic imaging.

Scenarios such as these have led to a continued interest in polarization enhancement techniques. The aim of these techniques is to increase the sensitivity of NMR and open up new applications of NMR that were previously impossible or infeasible due to sensitivity limitations. My research has focused on extending the technique of parahydrogeninduced polarization to heterogeneous hydrogenation reactions (ch. 2). Specifically, I have emphasized the application of this new technique to gas-phase imaging (ch. 3) and the characterization of micro-reactors (ch. 4-6). Although my research has focused on a rather narrow application of this new technique, I expect that the proof of principle experiments presented herein will lead to broader applications, such as the study of non-hydrogenation reactions, micro-imaging, biological imaging and other fields of magnetic resonance. 


\subsubsection{Basic Principles}

\section{Nuclear Magnetism}

The foundation of NMR rests on the concept of nuclear spin [138], i.e. the intrinsic angular momentum that each nucleus has independent of any physical motion, such as translation, vibration, rotation, etc. The total spin angular momentum of a nucleus is determined by its nuclear structure and has a value of $\hbar \sqrt{I(I+1)}$, where $I$ is restricted to half-integer values

$$
I=0, \frac{1}{2}, 1, \frac{3}{2}, \ldots
$$

The spin angular momentum provides a degree of freedom, $m_{I}=I, I-1, \ldots,-I+1,-I$, called the azimuthal quantum number, that is $2 I+1$-fold degenerate in the absence of magnetic and electric fields.

The power of magnetic resonance lies in the fact that the nucleus has a magnetic moment co-linear with the angular momentum and proportional to $m_{I}$,

$$
\mu=\hbar \gamma m_{I}
$$

where $\gamma$ is called the gyromagnetic ratio and is unique for each isotope. It is the interaction of this nuclear magnetic dipole with surrounding magnetic fields that lifts the degeneracy of the energy levels and provides a probe of the local nuclear environment. This sensitivity to the local magnetic field, $\vec{B}_{l o c}$, is what makes NMR so powerful in characterizing molecular structure and dynamics [101].

This introductory material will only address spin-1/2 nuclei, as my research has 
focused almost exclusively ${ }^{1}$ on the NMR and MRI of ${ }^{1} \mathrm{H}$, which is has a spin- $1 / 2$ nucleus.

\section{Zeeman Effect}

The Zeeman effect causes a splitting of the angular momentum energy levels due to the interaction of the nuclear magnetic moment with an applied magnetic field $[79,100,138]$,

$$
E=-\vec{\mu} \cdot \vec{B}=-\hbar \gamma m_{I} B_{0}= \pm \frac{1}{2} \hbar \gamma B_{0}
$$

where the axis of quantization is taken to be along $\vec{B}$ and, by convention, the static field is taken to be directed along the $z$-axis with magnitude $B_{0}$. Note that the low energy state for a spin- $1 / 2$ nucleus is given by $m_{I}=+\frac{1}{2}$ (denoted $|\alpha\rangle$ or $\left.|\uparrow\rangle\right)$, whereas the high energy state is given by $m_{I}=-\frac{1}{2}$ (denoted $|\beta\rangle$ or $\left.|\downarrow\rangle\right)$. The resulting energy difference, or Zeeman splitting, is

$$
\Delta E=E_{-1 / 2}-E_{+1 / 2}=\hbar \gamma B_{0}
$$

This energy splitting is important for two reasons. First, the Zeeman splitting leads to the formation of net magnetization, $M_{0}$, in the sample, and it is this macroscopic $M_{0}$ that is detected as the NMR signal. Second, the Zeeman interaction drives the equations of motion that allow for both the excitation and detection of the NMR signal. This is due to Schrödinger's relation that

$$
\hbar \frac{\mathrm{d} \psi}{\mathrm{d} t}=-\imath \mathcal{H} \psi
$$

\footnotetext{
${ }^{1}$ Several experiments were attempted or started which employed ${ }^{19} \mathrm{~F}$ or ${ }^{31} \mathrm{P}$, but due to catalyst and equipment failures (respectively), the experiments were not completed. In any event, these nuclei are both spin-1/2, so the only difference from ${ }^{1} \mathrm{H}$ NMR is the resonance frequency and the sensitivity.
} 


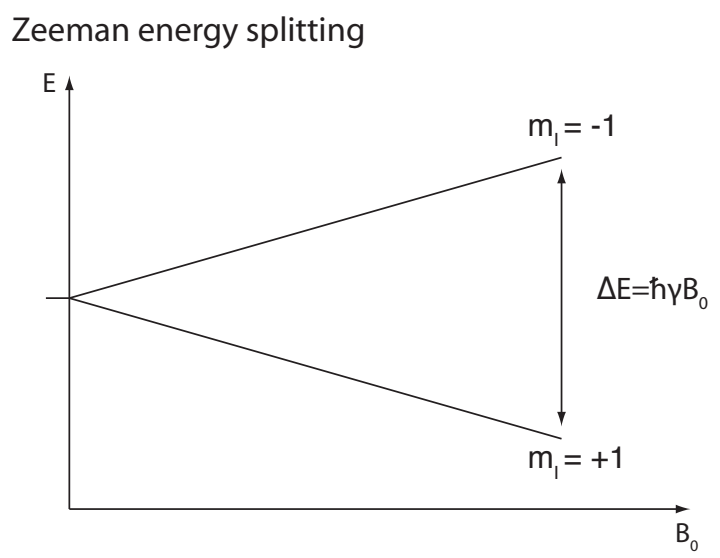

Figure 1.1: The energy levels associated with the $m_{I}$ become non-degenerate in a magnetic field. The energy splitting for a spin- $1 / 2$ nucleus is $\Delta E=\hbar \gamma B_{0}$

which is simply a statement that the time evolution of the quantum state, $\psi$, is governed by the energy interactions of the system, i.e. the energy operator, or Hamiltonian, $\mathcal{H}$ $[48,101,138]$.

\section{Equations of Motion and the NMR Signal}

The equations of motion for an ensemble of spins can be derived using classical concepts of gyromagnetism $[121,138]$ or directly from the evolution of the quantum states $[48,138]$. For an ensemble of isolated, non-interacting spins- $1 / 2$, the resulting motion is described by the Bloch equation,

$$
\frac{\mathrm{d} \vec{M}}{\mathrm{~d} t}=\vec{M} \times \gamma \overrightarrow{B(t)}-\frac{M_{x} \hat{x}+M_{y} \hat{y}}{T_{2}}-\frac{\left(M_{z}-M_{0}\right) \hat{z}}{T_{1}}
$$

The first term describes the precession around $\vec{B}$, the second terms describes the decay of the transverse magnetization and the last term describes the recovery of the magnetization along $\hat{z}$

Several important concepts are embodied in the Bloch equation. First, the pre- 
cessional motion of $M_{0}$ is in the plane perpendicular to $\vec{B}$ and has a frequency $\omega_{r f}=\gamma|B|$, called the Larmor frequency. This precessional motion is particularly important, as it is this rotating magnetization that is detected in $\mathrm{NMR}^{2}$ via Faraday's law of induction [121]. Furthermore, this precessional behavior is what requires oscillating magnetic fields for excitation and control of the nuclear spins [138]. Second, the relaxation terms describe the return of the system to equilibrium after excitation. By convention, the largest and static component of the magnetic field is taken to be the $\hat{z}$, or longitudinal, direction and the oscillating components of $M_{0}$ are in the $\hat{x}, \hat{y}$, or transverse, plane. Thus, the transverse magnetization decays with a rate, $T_{2}^{-1}$, while the longitudinal magnetization recovers to its thermal equilibrium value with a rate, $T_{1}^{-1}[54,89]$.

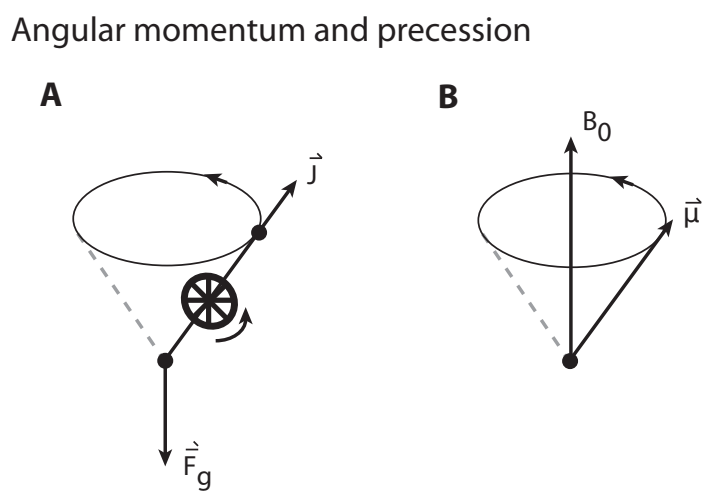

Figure 1.2: (A) A gyroscope precesses in a gravitational field because of the angular momentum provided by the rotating flywheel and the angular momentum provided by the torque resulting from the force of gravity. Conservation of angular momentum results in precessional motion in the plane perpendicular to the direction of the gravitational force. (B) A magnetic moment precesses in a magnetic field because of the intrinsic spin angular momentum and the torque applied by the interaction of the nuclear magnetic moment with the applied magnetic field. As in the case of the gyroscope, the precessional motion is perpendicular to magnetic field.

\footnotetext{
${ }^{2}$ Much of the NMR literature describes the physics of excitation and detection in terms of broadcasting/receiving radio frequency waves, but the signal detection actually occurs via magnetic induction. A fascinating discussion of the quantum origins of this signal is given by David Hoult [81-83].
} 


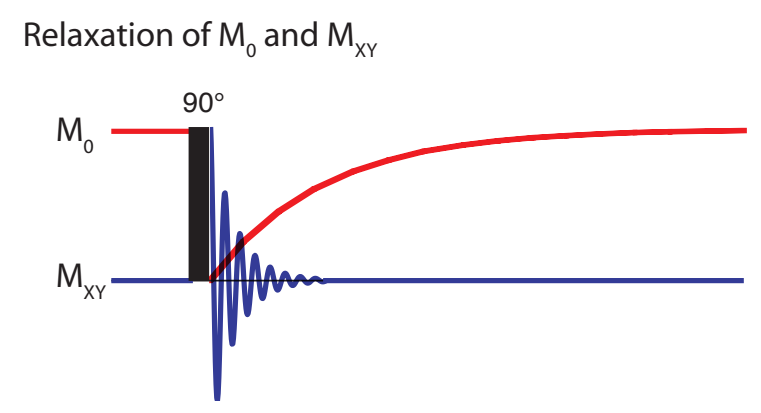

Figure 1.3: Following a $90^{\circ}$ pulse, the longitudinal magnetization, $M_{0}$ (red), is converted into transverse magnetization, $M_{X Y}$ (blue). $M_{X Y}$ decays exponentially to 0 with a rate $T_{2}^{-1}$. $M_{0}$ regrows along $\hat{z}$ with a rate $T_{1}^{-1}$.

\section{Other Interactions and Considerations}

It is important to note that other interactions exist in addition to the Zeeman interaction. These additional interactions perturb the Zeeman energy levels and, thus, influence the equation of motion. Furthermore, the macroscopic sample is heterogeneous on the molecular scale. A combination of quantum and statistical mechanics is needed to describe the evolution of the bulk NMR signal under more complicated interactions and sophisticated pulse sequences. This is accomplished via the density matrix formalism [31]. Specifically, the product operator formalism $[48,101]$ is very convenient for describing the behavior of multi-spin systems even when experiencing complicated interactions and pulse sequences.

The reader is referred to the literature for the details of density matrix theory and the application of product operators to NMR $[31,48,80,101]$. The concepts that will be used in this dissertation are,

- A spin operator is formed by the outer product of a ket and a bra, e.g. $\mathbf{I}_{\mathbf{z}}=\left|I_{z}\right\rangle\left\langle I_{z}\right|$ 
- The Hamiltonian operator is formed by replacing all the quantum operators in $\mathcal{H}$ with the corresponding spin operators.

- The state of the system is given by the density matrix, $\rho=|\psi\rangle\langle\psi|$

- The thermal equilibrium state is given by

$$
\rho_{\text {init }}=\frac{\exp (-\mathcal{H} / k T)}{\operatorname{Tr}(\exp (-\mathcal{H} / k T))}
$$

- The equation of motion is given by the Liouville-von Neumann eqn.

$$
\frac{\mathrm{d} \rho(t)}{\mathrm{d} t}=\imath[\rho(t),(H)]
$$

- Frame transformations are used to simplify the equations of motion, resulting in

$$
\mathcal{H}_{e f f}=\mathbf{U} \mathcal{H} \mathbf{U}^{-1}-\imath \mathbf{U} \frac{\mathrm{d}\left(\mathbf{U}^{-1}\right)}{\mathrm{d} t}
$$

Table 1.1 gives examples of the most important Hamiltonians for liquid state NMR.

The Zeeman interaction, be it with the static field or the RF field, is an interaction with an external magnetic field. The remaining nuclear spin interactions are due to the local magnetic fields arising from the surrounding nuclei and electrons. These interactions result in a considerable increase of spectral complexity and, thus, a wealth of knowledge about the nuclear environment $[1,101]$. Of these interactions, chemical shift, $\delta$, and $J$-coupling are of primary importance in liquid state NMR. 


$$
\begin{aligned}
\mathcal{H}_{Z} & =\sum_{i=1}^{N} \omega_{i} \mathbf{I}_{i z} \\
\mathcal{H}_{J} & =\sum_{i \neq j} \pi J_{i j} 2 \mathbf{I}_{i z} \mathbf{I}_{j z} \\
\mathcal{H}_{J, \mathrm{LF}} & =\sum_{i \neq j} \pi J_{i j}\left(2 \mathbf{I}_{i x} \mathbf{I}_{j x}+2 \mathbf{I}_{i y} \mathbf{I}_{j y}+\mathbf{I}_{i z} \mathbf{I}_{j z}\right) \\
\mathcal{H}_{r f} & =\gamma B_{1} \sum_{i=1}^{N}\left(\cos \phi \mathbf{I}_{i x}+\sin \phi \mathbf{I}_{i y}\right)
\end{aligned}
$$

Table 1.1: Useful Hamiltonians for Liquid State NMR. $\mathcal{H}_{Z}$ is the Zeeman Hamiltonian, $\mathcal{H}_{J}$ is the truncated J-coupling Hamiltonian (i.e. high field), $\mathcal{H}_{J \text {,LF }}$ is the untruncated Jcoupling Hamiltonian (i.e. low field), and $\mathcal{H}_{r f}$ is the Hamiltonian for a hard pulse with phase $\phi$.

\subsubsection{Sensitivity and Nuclear Spin Polarization}

\section{Signal to Noise Ratio}

As mentioned in sec. 1.1.1, the advantages of NMR are offset by its inherent low sensitivity. This problem of low sensitivity has received much scrutiny over the past 60 years and has been the driving force behind developments in techniques such as moving from continuous-wave to Fourier Transform (FT) NMR, the move from permanent magnets to ever more powerful superconducting magnets, and the recent push for cryogenically cooled receiver coils and pre-amplifiers to name just the more prominent examples [51].

In order to find a clever way around this sensitivity problem, it is essential to understand what factors influence the signal to noise ratio (SNR). The SNR for NMR is 
typically given as $[48,121]$

$$
\mathrm{SNR} \propto \frac{N \gamma^{5 / 2} B_{0}^{3 / 2}}{T^{3 / 2}} \sqrt{\frac{N_{s} T_{\text {read }}}{T_{R}}} \delta_{\mathrm{Hz}}
$$

where $N$ is the number of spins with gyromagnetic ratio $\gamma$, at thermal equilibrium in a magnetic field $B_{0}$, with the sample and RF coil held at temperature $T . N_{s}$ is the number of signal averaging scans, $T_{\text {read }}$ is the acquisition time, $T_{R}$ is the repetition time and $\delta_{H z}$ is the spectral resolution. Equation 1.7 explains

- the tradeoff between SNR and resolution, $\delta_{\mathrm{Hz}}$, i.e., $\mathrm{SNR} \propto \delta_{\mathrm{Hz}}$,

- that signal averaging is time consuming, i.e., $\mathrm{SNR} \propto N_{s}^{1 / 2}$,

- the push for larger field strengths, i.e., $\mathrm{SNR} \propto B_{0}^{3 / 2}$,

- the predominance of ${ }^{1} \mathrm{H}$ NMR, i.e., it has one of the largest $\gamma$ 's and $100 \%$ natural abundance provides a large $N$,

- the infrequent use of gaseous samples, e.g., comparing the densities of acetylene gas $\left(\mathrm{C}_{2} \mathrm{H}_{2}\right)$ and liquid water $\left(\mathrm{H}_{2} \mathrm{O}\right)$ at room temperature, $N_{a} / N_{w}=0.0011$; a SNR drop of 3 orders of magnitude!

Note that the temperature, $T$, is not typically used to optimize the SNR, as it is restricted to a relatively narrow range based on the sample being studied.

\section{Separating the Polarization and Detection Steps}

Equation 1.7 contains several built-in assumptions. Specifically, it assumes that the sample is thermally polarized, that the polarization and detection occur at the same 
field strength and temperature, that the sample and the receiver coil are at the same temperature, that the noise is coil dominated and not sample dominated, ${ }^{3}$ and that no polarization was transferred between spins with different $\gamma$. These assumptions seem quite valid and, indeed, this is the case for most simple NMR experiments. In order to more strategically optimize the SNR, the dependence of the SNR on these assumptions must be determined. The magnitude of the signal equation [121],

$$
|S(t)| \propto \frac{B_{1}}{1 \mathrm{~A}} M_{0} \omega_{d}
$$

shows that the signal strength depends on three factors: the sensitivity of the receiver coil, $B_{1} / 1 \mathrm{~A}$; the net magnetization, $M_{0}$; and the frequency at which the detection occurs, $\omega_{d}$ (due to Faraday's law of induction). Furthermore, the noise amplitude in a circuit tuned to resonate at the detection frequency is $\propto \sqrt{T_{c} \gamma_{d} B_{0}}$. This yields

$$
\mathrm{SNR} \propto \overbrace{\left(\frac{N \gamma_{p}^{2} B_{p}}{T}\right)}^{\mathrm{SNR}_{\mathrm{p}}} \overbrace{\sqrt{\frac{\gamma_{d} B_{0}}{T_{c}}}}^{\mathrm{SNR}_{\mathrm{d}}}
$$

Using eq. 1.9, the polarization and detection steps $\left(\mathrm{SNR}_{\mathrm{p}}\right.$ and $\mathrm{SNR}_{\mathrm{d}}$ respectively) can be optimized independently. This has led to a variety of clever methods for improving the sensitivity, as discussed below.

For improving the sensitivity of the detection step, the most common technique is referred to as inverse detection [51] and exploits the large $\gamma$ of ${ }^{1} \mathrm{H}$ for detecting the evolution of lower $\gamma$ (and lower natural abundance) species such as ${ }^{13} \mathrm{C}$. The more recent development of cryo-probes exploits the $T_{c}$ dependence and reduces the noise amplitude by cooling down

\footnotetext{
${ }^{3}$ Coil noise dominance is the typical situation in NMR and materials imaging, whereas body noise dominance is the typical case for medical MRI. This is due to the RF noise created by the thermal motion of ions dissolved in body fluids.
} 
the receiver coil to cryogenic temperatures (as well as the first stage preamplifiers). Another approach to improving the $\mathrm{SNR}_{d}$ is to eliminate inductive detection altogether. Alternatives to inductive detection are superconducting quantum interference devices (SQUIDs) $[99,145]$ and laser detection via atomic magnetometry [156]. Both techniques are being actively pursued here at UC Berkeley. These techniques have completely different noise behavior than that given in eq. 1.9, but typically only provide better SNR than inductive detection at lower magnetic field strengths.

Optimization of the polarization step is most commonly performed by transferring the relatively large thermal polarization of ${ }^{1} \mathrm{H}$ to nuclei with lower $\gamma$ and/or low natural abundance via $\mathrm{INEPT}^{4}$ or $\mathrm{CP}^{5}[51,125]$. It is also possible to use a pre-polarizing field, $B_{p}$, to achieve a thermal polarization larger than that supplied by $B_{0}$, but this is typically only done in situations where $B_{0}$ is very small and an electromagnet can be used for $B_{p}$ [150]. As with the detection step, $\mathrm{SNR}_{p}$ can be completely replaced by a different expression if some method other than the Zeeman effect is used to polarize the nuclear spins. These approaches are referred to as hyperpolarization techniques.

\section{Net Magnetization, Spin Polarization and HyperPolarization}

A definition of $M_{0}$ is necessary for understanding hyperpolarization:

$$
M_{0}=\hbar \gamma \sum_{m=-I}^{I} m_{I} N_{m}=N \hbar \gamma \sum_{m=-I}^{I} m_{I} P_{m}=N \hbar \gamma\left\langle m_{I}\right\rangle=N \hbar \gamma I p
$$

where $m_{I}$ is the azimuthal quantum number, $N_{m}$ is the number of nuclei in the ensemble in the $m_{I}$ state, $P_{m}$ is the probability of a nucleus being in the $m_{I}$ state and $p$ is the nuclear

\footnotetext{
${ }^{4}$ Insensitive $N$ uclei Enhancement by Polarization Transfer

${ }^{5}$ Cross Polarization
} 
spin polarization, defined so that $0 \leq p \leq 1$. The magnitude of $M_{0}$ is governed by the relative population distribution of the Zeeman energy levels, $\left\langle m_{I}\right\rangle$. The magnitude of $M_{0}$ has a maximum value when all of the nuclei are in the $m_{I}=+I$ or $-I$ state and is 0 if all the Zeeman levels are equally populated. This behavior of $M_{0}$ is summarized in the concept of nuclear spin polarization, $p=\left|\left\langle m_{I}\right\rangle\right| / I$ so that maximum signal is obtained for a polarization of $100 \%$, no signal is observed for a polarization of $0 \%$, etc.

At thermal equilibrium, the spin polarization is calculated using the Boltzmann distribution to solve for,

$$
\left\langle m_{I}\right\rangle=\frac{\sum_{m=-I}^{I} m_{I} P_{m}}{\sum_{m=-I}^{I} P_{m}}=\frac{\sum_{m=-I}^{I} m_{I} \exp \left(-E_{m} / k T\right)}{\sum_{m=-I}^{I} \exp \left(-E_{m} / k T\right)}
$$

Using eq. 1.3 for $E_{m}$, the characteristic temperature is given by the energy difference of two adjacent Zeeman levels,

$$
\theta_{Z}=\frac{\Delta E_{m}}{k}=\frac{\hbar \gamma B_{0}}{k}
$$

$\theta_{Z}=14 \mathrm{mK}$ for ${ }^{1} \mathrm{H}$ at $7.0 \mathrm{~T}$ and the polarization is given by

$$
\begin{aligned}
p=\frac{\left\langle m_{I}\right\rangle}{I} & =\frac{\exp \left(\frac{\theta_{Z}}{2 T}\right)-\exp \left(\frac{\theta_{Z}}{2 T}\right)}{\exp \left(\frac{\theta_{Z}}{2 T}\right)+\exp \left(\frac{\theta_{Z}}{2 T}\right)}=\frac{\sinh \left(\frac{\theta_{Z}}{2 T}\right)}{\cosh \left(\frac{\theta_{Z}}{2 T}\right)} \\
& =\tanh \left(\frac{\theta_{Z}}{2 T}\right) \approx \frac{\theta_{Z}}{2 T}=\frac{\hbar \gamma B_{0}}{2 k T}
\end{aligned}
$$

which is the value used in $M_{0}$ in eq. 1.9,

$$
M_{0}=\frac{N \hbar \gamma}{2} p=\frac{N \hbar^{2} \gamma^{2} B_{0}}{4 k T}
$$

The linear approximation of $p$ is accurate for all reasonable magnetic field strengths and temperatures. ${ }^{6}$

\footnotetext{
${ }^{6} B_{0}$ must be larger than $10^{6} \mathrm{~T}$ or the temperature less than $1 \mathrm{~K}$ before significant deviations from linearity are observed.
} 


\section{Nuclear spin polarization}

A

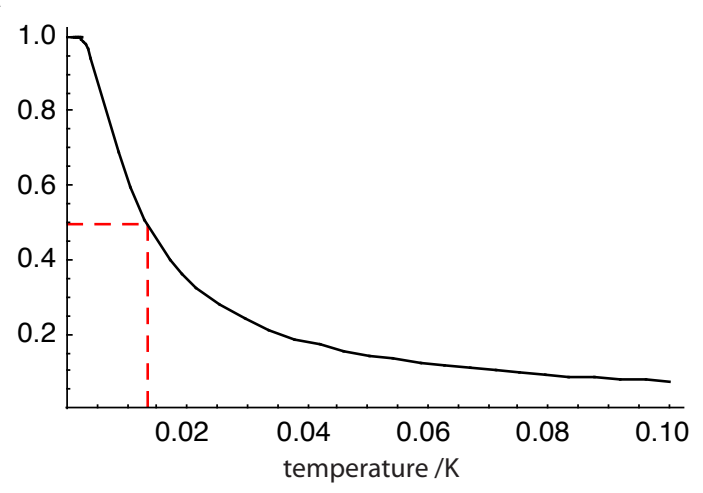

B

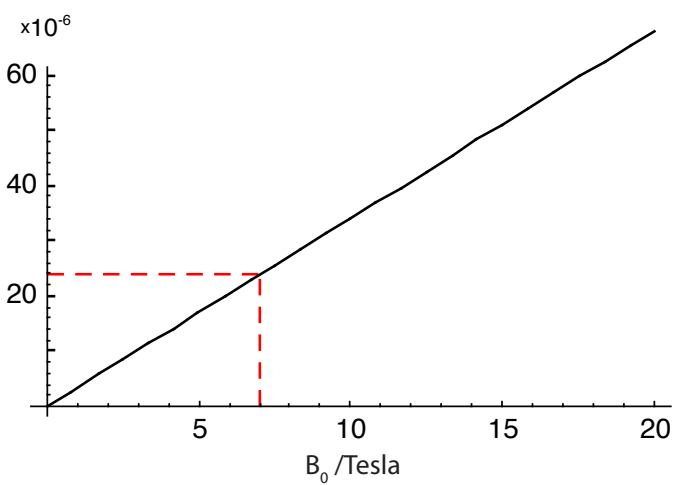

Figure 1.4: Behavior of the thermal equilibrium nuclear spin polarization for ${ }^{1} \mathrm{H}$. (A) Temperature dependence of the polarization. $\theta_{Z}=14 \mathrm{mK}$ at $B_{0}=7.0 \mathrm{~T}$, requiring temperatures less than a fraction of a $\mathrm{K}$ to obtain polarizations above $1 \% . \theta_{Z}=14 \mathrm{mK}$. (B) Field dependence of the polarization. Magnetic field strengths on the order of $10^{4}$ are required to obtain polarizations above $1 \%$. Our experiments were performed at $7.05 \mathrm{~T}$.

The key point in the discussion above is that the polarization at thermal equilibrium is very small, $p=2.3 \times 10^{-5}$ for ${ }^{1} \mathrm{H}$ at $300 \mathrm{~K}$ and $7.0 \mathrm{~T}$. This is due to the fact that the Zeeman energy splitting is much smaller than the thermal energy for $T \gtrsim 1 \mathrm{~K}$. As shown in fig. 1.4, $\theta_{Z}$ is a good measure of the temperature at which the equilibrium polarization $\sim 1$

The goal of hyperpolarization techniques is to manipulate the populations of the Zeeman levels and generate polarizations larger than those available at readily accessible temperatures. The key to achieving hyperpolarization is to exploit some interaction that couples the nuclear spin angular momentum to some other degree of freedom that is easier to control. For example, hyperpolarization of noble gases is obtained by optically pumping a specific electronic transition of an alkali vapor. Under the right conditions [41,71], the relaxation of this excited state couples to a nearby nuclear spin and causes a spin flip. Over 
time, this builds up a net population difference leading to a large nuclear spin polarization. Other examples of hyperpolarization techniques include dynamic nuclear polarization (DNP), which exploits the very large Zeeman splitting of electrons and uses dipolar couplings to transfer this polarization to nuclei in the vicinity of the radical [1]; chemical induced dynamic nuclear polarization (CIDNP), which uses the generation of radical and the hyperfine coupling to generate polarization [65]; and parahydrogen-induced polarization (PHIP), which uses the symmetry properties of $\mathrm{H}_{2}$ to generate large polarizations after appropriate hydrogenation of a substrate [37,127].

Extending the application of parahydrogen-induced polarization is the focus of my research. In the following sections, I describe some basic details of ortho- and parahydrogen, how they are used to create a hyperpolarized state and, finally, a brief review of the applications of PHIP.

\subsection{Ortho- and Parahydrogen}

\subsubsection{Consequences of Symmetry}

In addition to the interactions mentioned above (sec. 1.1.2), the behavior of nuclei are also dictated by one of two statistical laws, based on their spin quantum number $[79,100]$. Nuclei with whole integer spin, $I=0,1,2, \ldots$, are called bosons and must obey BoseEinstein statistics, whereas nuclei with half integer spin, $I=\frac{1}{2}, \frac{3}{2}, \frac{5}{2}, \ldots$ are called fermions and are required to obey Fermi-Dirac statistics. ${ }^{1} \mathrm{H}$ nuclei, being spin- $1 / 2$, must conform to Fermi-Dirac statistics, which requires that the total wavefunction be antisymmetric under 
exchange of two fermions,

$$
\Psi_{\text {tot }}(A, B)=-\Psi_{\text {tot }}(B, A)
$$

The consequences of this symmetry requirement can be seen by applying the BornOppenheimer approximation [132] and expanding the total wavefunction into a product of wavefunctions for each degree of freedom. This approximation is excellent in the case of $\mathrm{H}_{2}$,

$$
\Psi_{t o t}=\psi_{t} \psi_{e} \psi_{v} \psi_{r} \psi_{n s}
$$

The translational, (ground state) electronic and vibrational wavefunctions are all symmetric with respect to exchanging the $\mathrm{H}_{2}$ nuclei $[79,100]$, while the rotational wavefunction contains states that are either symmetric or antisymmetric under this exchange. Specifically, the rotational states with $j=0,2,4, \ldots$ are symmetric, while the $j=1,3,5, \ldots$ states are antisymmetric under exchange of the nuclei. This leaves the nuclear spin wavefunction, which is required to be either symmetric or antisymmetric in order to satisfy the symmetry requirements of $\Psi_{t o t}$.

Given that $\psi_{n s}$ must be symmetric or antisymmetric under exchange of the nuclei, the simple product basis is not appropriate,

$$
\begin{aligned}
& |\alpha \alpha\rangle=|\alpha \alpha\rangle \quad|\alpha \beta\rangle \neq \pm|\beta \alpha\rangle \\
& |\beta \beta\rangle=|\beta \beta\rangle \quad|\beta \alpha\rangle \neq \pm|\alpha \beta\rangle
\end{aligned}
$$




\begin{tabular}{c|ccc|cll}
$\Psi_{\text {tot }}$ & $\mathrm{j}$ & $\psi_{r}$ & $m_{I_{\text {tot }}}$ & $\psi_{n s}$ & & \\
\hline $\mathrm{a}$ & even & $\mathrm{s}$ & 0 & $\mathrm{a}$ & $\left|S_{0}\right\rangle$ & $p-\mathrm{H}_{2}$ \\
$\mathrm{a}$ & odd & $\mathrm{a}$ & 1 & $\mathrm{~s}$ & $\left|T_{+1}\right\rangle$ & $o-\mathrm{H}_{2}$ \\
$\mathrm{a}$ & odd & $\mathrm{a}$ & 0 & $\mathrm{~s}$ & $\left|T_{0}\right\rangle$ & $o-\mathrm{H}_{2}$ \\
$\mathrm{a}$ & odd & $\mathrm{a}$ & -1 & $\mathrm{~s}$ & $\left|T_{-1}\right\rangle$ & $o-\mathrm{H}_{2}$ \\
\hline
\end{tabular}

Table 1.2: The nuclei of $\mathrm{H}_{2}$ are fermions and the resulting symmetry requirements couple the rotational and nuclear spins wavefunctions together.

However, appropriate linear combinations of these states provide a suitable basis set,

$$
\begin{aligned}
& \left|S_{0}\right\rangle_{\alpha, \beta}=\frac{1}{\sqrt{2}}(|\alpha \beta\rangle-|\beta \alpha\rangle)=-\left|S_{0}\right\rangle_{\beta, \alpha} \\
& \left|T_{+1}\right\rangle_{\alpha, \beta}=|\alpha \alpha\rangle \quad=\left|T_{+1}\right\rangle_{\beta, \alpha} \\
& \left|T_{0}\right\rangle_{\alpha, \beta}=\frac{1}{\sqrt{2}}(|\alpha \beta\rangle+|\beta \alpha\rangle)=\left|T_{0}\right\rangle_{\beta, \alpha} \\
& \left|T_{-1}\right\rangle_{\alpha, \beta}=|\beta \beta\rangle \quad=\left|T_{-1}\right\rangle_{\beta, \alpha}
\end{aligned}
$$

The antisymmetric state is a singlet state with total angular momentum, $I_{t o t}=0$ and is given the name parahydrogen $\left(p-\mathrm{H}_{2}\right)$. The three symmetric states form a triplet state, with $I_{t o t}=1$, and are called orthohydrogen $\left(o-\mathrm{H}_{2}\right)$. From an NMR perspective, the need for a symmetric/antisymmetric basis can also be argued on the principle of strong coupling [101], i.e. the nuclei in $\mathrm{H}_{2}$ are magnetically equivalent, so $\Delta \omega=0$, satisfying $\Delta \omega \ll 2 \pi|\mathbf{J}|$, and the resulting eigenstates of the strong coupling Hamiltonian are the same as the symmetric/antisymmetric basis.

Table 1.2 summarizes these results. This is a fascinating result — when the molecule occupies a symmetric rotational state, $\psi_{n s}$ is required to be antisymmetric, while an antisymmetric rotational state is forced to occupy a symmetric $\psi_{n s}$. The rotational and nuclear spin degrees of freedom are coupled together purely by symmetry requirements, 
not by any physical force. In practical terms, this is appealing because this symmetrybased coupling allows us to control the nuclear spin state by manipulating the manifold of rotational states, precisely what is required for a hyperpolarization technique.

\subsubsection{Manipulating the Fraction of Parahydrogen}

The idea of using the rotational states of $\mathrm{H}_{2}$ to control the populations of the nuclear spin states is appealing for several reasons. First, the rotational states are easier to manipulate via changes in temperature due to the larger energy spacings. This is clear from the larger characteristic temperature ${ }^{7}$,

$$
\begin{aligned}
E_{R}(j) & =\frac{\hbar^{2}}{2 I} j(j+1) \\
\theta_{R} & =\frac{\hbar^{2}}{2 I k} \approx 85.3 \mathrm{~K}
\end{aligned}
$$

which is almost four orders of magnitude larger that $\theta_{Z}$. This is depicted in figs. 1.5 and 1.6, where it is also evident that the ground rotational state is $p-\mathrm{H}_{2}$. This is the second reason this strategy is so useful, i.e. by cooling $\mathrm{H}_{2}$ gas to a $T \approx \theta_{R}$ we expect the fraction of $\mathrm{H}_{2}$ molecules in the $p-\mathrm{H}_{2}$ nuclear spin state to approach unity. Because $p-\mathrm{H}_{2}$ is a pure nuclear spin state, the result is a very high degree of polarization.

As in sec. 1.1.3, the exact percentage of $\mathrm{H}_{2}$ molecules in the $p-\mathrm{H}_{2}$ state at a given temperature is calculated using the partition function and eq. 1.21:

$$
f=\frac{\sum_{\text {even } j}(2 j+1) \exp \left(-\frac{\theta_{R}}{T} j(j+1)\right)}{\sum_{\text {even } j}(2 j+1) \exp \left(-\frac{\theta_{R}}{T} j(j+1)\right)+3 \sum_{\text {odd } j}(2 j+1) \exp \left(-\frac{\theta_{R}}{T} j(j+1)\right)}
$$

\footnotetext{
7 The formula given here actually evaluates to $\approx 87.5 K$, whereas calculating $\theta_{R}$ from measured rotational constants give $\theta_{R}=\frac{h c B}{k} \approx 85.3 \mathrm{~K}$, the discrepancy is due to our assumption that each degree of freedom is completely independent, in reality there is coupling between the rotational and vibrational states.
} 


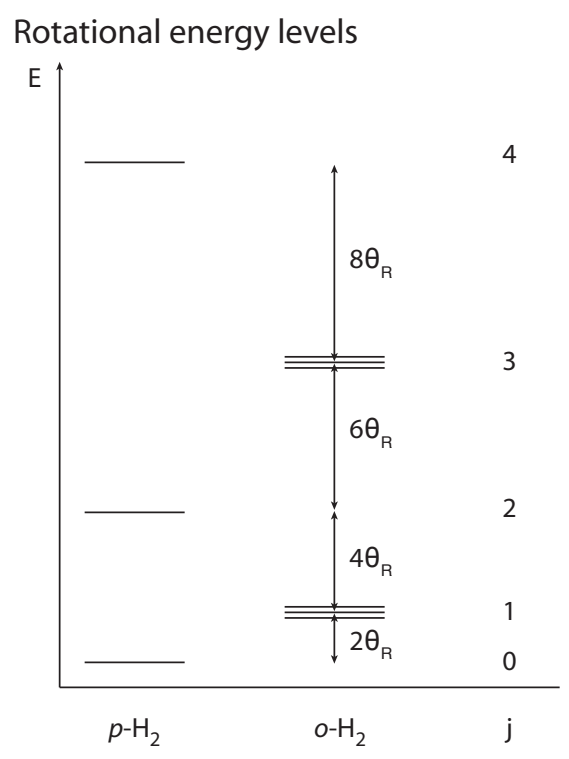

Figure 1.5: The rotational energy levels are separated by $2 j \theta_{R}$ and the odd rotational levels have a 3 -fold degeneracy due to the three $m_{I}$ states of $o-\mathrm{H}_{2}$. In the limit of $T \ll \theta_{R}$ only $j=0$ will be populated, resulting in $100 \% p-\mathrm{H}_{2}$.

where the degeneracy of the $o-\mathrm{H}_{2}$ states must be included when summing over the odd rotational levels. The result is shown in fig. 1.7.

Note that $f>98 \%$ for $T<25 \mathrm{~K}, f \approx 50 \%$ when $T=77 \mathrm{~K}$, and $f=25 \%$ for $T>$ 225K. 77 and $25 \mathrm{~K}$ are much easier to obtain than the $\mathrm{mK}$ temperatures required to produce significant polarization via the Zeeman splitting (fig. 1.6). Specifically, temperatures $\geq 77 \mathrm{~K}$ are easily reached, as this is the boiling point of liquid $\mathrm{N}_{2}$, and temperatures near $25 \mathrm{~K}$ are relatively easy to reach using standard compressed He cryostats. A discussion of the equipment that $\mathrm{I}$ have built to $\mathrm{cool} \mathrm{H}_{2}$ gas to $15 \mathrm{~K}<T<77 \mathrm{~K}$ is found in sec. A.1.

The discussion above has focused solely on the thermodynamics of the rotational and nuclear spin states. For this technique to be practical, the kinetics of the $o-\mathrm{H}_{2}$ to $p-\mathrm{H}_{2}$ conversion must also be favorable. However, it turns out that the transition from 
A 2 spin, AX system

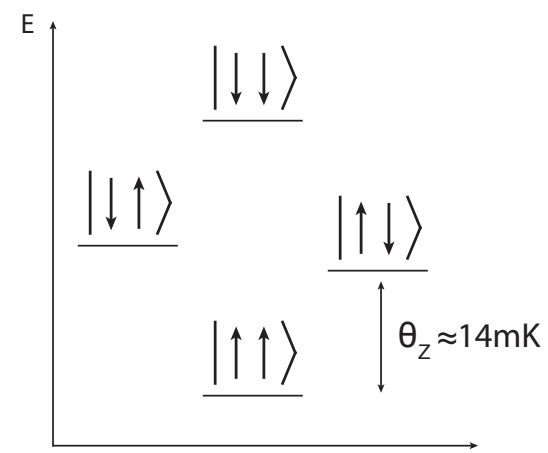

B 2 spin, $A_{2}$ system

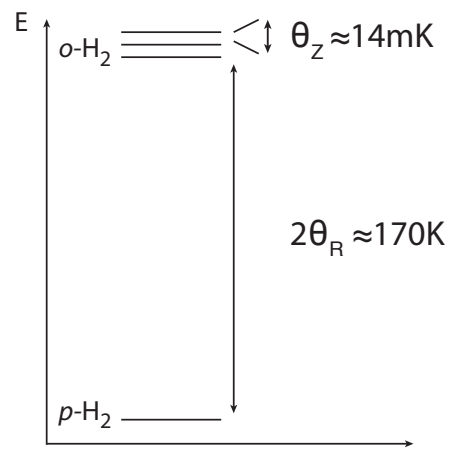

Figure 1.6: (A) The eigenstates of the Zeeman interaction are the simple product states, with energy spacings on the order of $14 \mathrm{mK}$ (at 7.0T). (B) The eigenstates corresponding to $p-\mathrm{H}_{2}$ and $o-\mathrm{H}_{2}$ are the symmetric and antisymmetric states, separated by $2 \theta_{R} \approx 170 \mathrm{~K}$ with the $o-\mathrm{H}_{2}$ states further split by the Zeeman interaction.

$o-\mathrm{H}_{2}$ to $p-\mathrm{H}_{2}$ is forbidden by angular momentum selection rules $[79,100]$, resulting in an exceedingly slow conversion. The hyperfine coupling of the $\mathrm{H}_{2}$ nuclear spins to the electrons allows this transition to occur during a collision, but the coupling is very weak and this form of conversion occurs on a timescale of months [100]. Luckily, a variety of ortho/para catalysts exist that lead to fast equilibration of the nuclear spin states to their equilibrium populations $[56,147]$. Section A.1 discusses in detail the conversion cells that I designed and built for creating a constant flow of $p-\mathrm{H}_{2}$ equilibrated to temperatures $\leq 77 \mathrm{~K}$. 
$\% p-\mathrm{H}_{2}$ vs. temperature

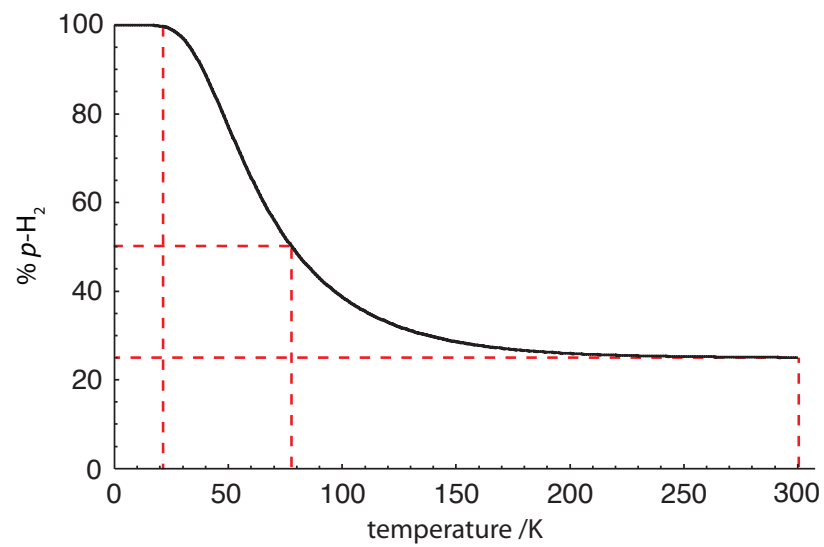

Figure 1.7: The fraction of $p-\mathrm{H}_{2}$ at thermal equilibrium vs. temperature. The temperatures used in this work are room temperature, $77 \mathrm{~K}$, and $25 \mathrm{~K}$. The resulting fractions of $p-\mathrm{H}_{2}$ are approximately $98 \%, 50 \%$, and $25 \%$, respectively.

\subsection{Parahydrogen-induced Polarization}

In order to discuss $o-\mathrm{H}_{2}$ and $p-\mathrm{H}_{2}$ and the details leading to polarization enhancement, it is convenient to use the product operator notation [10]:

$$
\begin{gathered}
\mathbf{T}_{ \pm 1}=\left|T_{ \pm 1}\right\rangle\left\langle T_{ \pm 1}\right|=\frac{\mathbf{1}_{4}}{4}+\frac{1}{2}\left( \pm \mathbf{I}_{z} \pm \mathbf{S}_{z}+2 \mathbf{I}_{z} \mathbf{S}_{z}\right) \\
\mathbf{T}_{0}=\left|T_{0}\right\rangle\left\langle T_{0}\right|=\frac{\mathbf{1}_{4}}{4}+\left(\mathbf{I}_{x} \mathbf{S}_{x}+\mathbf{I}_{y} \mathbf{S}_{y}-\mathbf{I}_{z} \mathbf{S}_{z}\right) \\
\mathbf{S}_{0}=\left|S_{0}\right\rangle\left\langle S_{0}\right|=\frac{\mathbf{1}_{4}}{4}-\left(\mathbf{I}_{x} \mathbf{S}_{x}+\mathbf{I}_{y} \mathbf{S}_{y}+\mathbf{I}_{z} \mathbf{S}_{z}\right)
\end{gathered}
$$

Using these equations, it is easily shown that a mixture of $o-\mathrm{H}_{2}$ and $p-\mathrm{H}_{2}$ produces an initial state of

$$
\rho_{\text {mix }}=\frac{\mathbf{1}_{4}}{4}+\left(\frac{1-4 f}{3}\right)\left(\mathbf{I}_{x} \mathbf{S}_{x}+\mathbf{I}_{y} \mathbf{S}_{y}+\mathbf{I}_{z} \mathbf{S}_{z}\right)=\frac{\mathbf{1}_{4}}{4}+\mathbf{I} \cdot \mathbf{S}
$$

where $f$ is the mol $\%$ of $p-\mathrm{H}_{2}$ and $(1-f)$ is the mole $\%$ of $o-\mathrm{H}_{2}$ in the gas mixture. It should be noted that if the gas mixture has been stored in a high magnetic field for a 
time longer than $T_{1}$, the Zeeman states of $o-\mathrm{H}_{2}$ will be populated and $\rho_{\text {mix }}$ will have an

additional term, $\epsilon\left(\frac{1-f}{3}\right)\left(\mathbf{I}_{z}+\mathbf{S}_{z}\right)$, where $\epsilon \propto p \approx 10^{-5}$ is a very small perturbation on the same order as thermal polarization.

\subsubsection{Creating Observable Net Magnetization}

Sections 1.2 and A.1 demonstrate that it is relatively easy to generate a highly polarized nuclear spin state in the form of $p-\mathrm{H}_{2}$. However, $p-\mathrm{H}_{2}$ has total spin angular momentum of 0 and, like a spin- 0 nucleus (e.g. ${ }^{12} \mathrm{C}$ ), it is NMR inactive. An alternative explanation uses the product operator expression for $p-\mathrm{H}_{2}$ given in eq. 1.24. Using the commutator relationships for product operators [48], it is straightforward to show that

$$
\left[\rho_{\text {mix }}, \mathbf{I}_{i}+\mathbf{S}_{i}\right]=0
$$

for $i=x, y, z$. This means that a mixture of $\mathrm{H}_{2}$ gas, regardless of its polarization, commutes with both the excitation Hamiltonian, $\mathcal{H}_{r f}$, and the liquid state Hamiltonian, $\mathcal{H}_{Z}+\mathcal{H}_{J}$ $[10,11,28,140]$. In other words, this state is completely immune to any pulse sequence or evolution time applied to it.

In order to utilize this highly polarized state, it must be made observable. Note that the relationship in eq. 1.26 does not hold when $\omega_{I} \neq \omega_{S}$,

$$
\left[\rho_{\text {mix }}, \mathbf{I}_{i}-\mathbf{S}_{i}\right]=\imath\left(2 \mathbf{I}_{j} \mathbf{S}_{k}-2 \mathbf{I}_{k} \mathbf{S}_{j}\right)
$$

Creating a chemical shift difference allows $\rho_{\text {mix }}$ to evolve under both $B_{0}$ and $B_{1}$. Specifically, rotation by $B_{1}$ (i.e. $i=\hat{x}$ or $i=\hat{y}$ ) creates observable, antiphase terms (e.g. $\mathbf{I}_{y} \mathbf{S}_{z}, \mathbf{I}_{z} \mathbf{S}_{y}$, etc.) $[36,118]$. One way to make the two nuclear spins inequivelent is to alter the electronic 


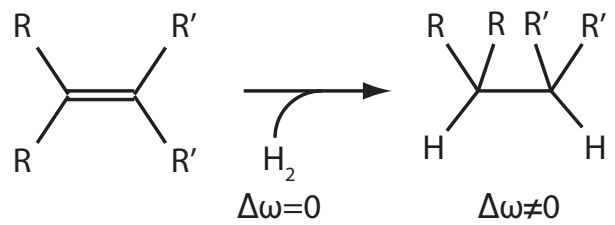

Figure 1.8: To observe the highly polarized $p-\mathrm{H}_{2}$ state, it must be transferred to magnetically inequivelent positions on a molecule via molecular addition. This is easily accomplished with many homogeneous catalysts, but traditional (dispersed metal) heterogeneous catalysts break the molecular hydrogen into atomic hydrogen, destroying the correlation and the resulting polarization.

structure at those sites; i.e., attach the $\mathrm{H}_{2}$ to a molecule. Molecular addition across a double bond is the most common way to achieve this result. The key requirements are that the hydrogenation occurs via molecular addition so that the two nuclear spins remain correlated; the hydrogenation occurs quickly, to prevent the correlated state relaxing during the intermediate steps of the reaction; and the reaction places the H's in magnetically inequivalent positions. The two most common implementations of this approach are termed PASADENA and ALTADENA and are described below. The more generic term used for all forms of polarization derived from mixtures of $o-\mathrm{H}_{2}$ and $p-\mathrm{H}_{2}$ is $p$ arahydrogen $i$ nduced polarization (PHIP).

\subsubsection{PASADENA}

PASADENA stands for parahydrogen and synthesis allow dramatic enhancement of nuclear alignment. This effect was initially observed and misinterpreted as CIDNP [134]. It was later predicted [37] and then experimentally verified [38,62].

If the hydrogenation step is carried out inside the bore of the magnet, the situation is well approximated by an instantaneous change of the nuclear spin Hamiltonian from the 
PASADENA

A

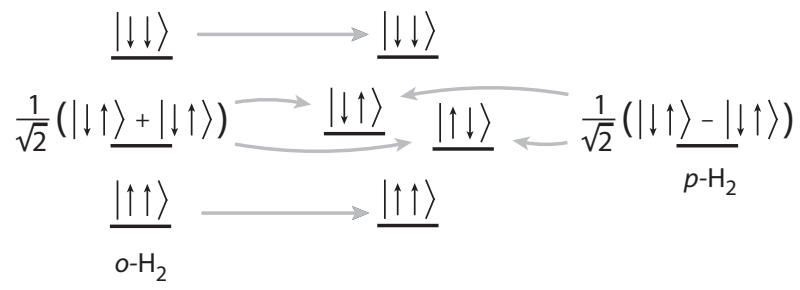

B
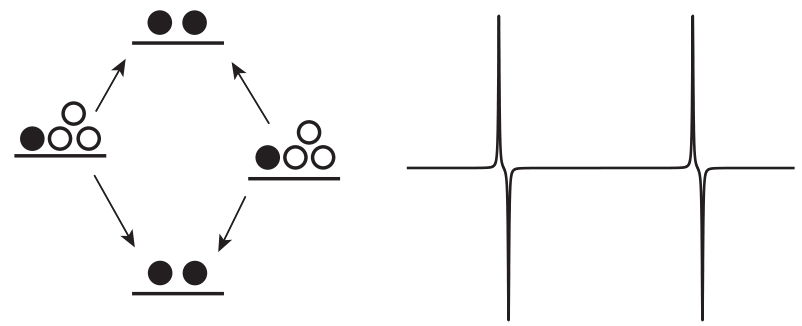

Figure 1.9: The PASADENA experiment. Hydrogenation occurs inside the bore of the magnet. (A) The sudden change in the Hamiltonian projects the singlet state onto the high field eigenstates. (B) The expected spectrum consists of anti-phase doublets.

strongly coupled $\mathrm{A}_{2}$ to the weakly coupled AX Hamiltonian. Mathematically speaking, the sudden approximation is used to obtain the resulting states by projecting $\rho_{0}$ onto the eigenstates of $\mathrm{AX}$, as shown in fig. 1.9. $\mathrm{H}_{2}$ gas containing a net excess of $p-\mathrm{H}_{2}$ relative to $n-\mathrm{H}_{2}$ will result in excess population of the central, $m_{I}=0$, energy levels. Because of this, the resulting spectrum is composed of distinctive anti-phase doublets.

While the energy level diagrams predict the correct qualitative aspects of the received signal, a product operator calculation is necessary for obtaining the dependence of the resulting spectrum on the flip angle, $\theta$, of the RF pulse, as well as the J-coupling topology [36,118]. Another important result that is most easily explained via use of the product operator notation is the difference between incoherent and coherent PASADENA $[10]$. 
When a molecule is hydrogenated in a magnetic field, the resulting state, eq 1.25 begins to evolve. Specifically,

$$
\begin{aligned}
& \mathbf{I}_{x} \mathbf{S}_{x} \rightarrow\left(\cos \left(\omega_{I} t\right) \mathbf{I}_{x}+\sin \left(\omega_{I} t\right) \mathbf{I}_{y}\right)\left(\cos \left(\omega_{S} t\right) \mathbf{S}_{x}+\sin \left(\omega_{S} t\right) \mathbf{S}_{y}\right) \\
& \mathbf{I}_{y} \mathbf{S}_{y} \rightarrow\left(\cos \left(\omega_{I} t\right) \mathbf{I}_{y}+\sin \left(\omega_{I} t\right) \mathbf{I}_{x}\right)\left(\cos \left(\omega_{S} t\right) \mathbf{S}_{y}+\sin \left(\omega_{S} t\right) \mathbf{S}_{x}\right)
\end{aligned}
$$

Under typical conditions, many molecules are formed at random initial times. This lack of phase coherence between the molecules leads to destructive interference and the cancellation

of the terms in eq. 1.28 , leaving $\rho_{\text {mix }}=\frac{\mathbf{1}_{4}}{4}-\mathbf{I}_{z} \mathbf{S}_{z}$. Traditionally, this is the situation referred to as PASADENA.

A variation of PASADENA that retains the phase coherence of all the hydrogenated molecules is called coherent PASADENA. This can be accomplished by precise control of the reaction, for example by initiating the reaction with a short laser pulse [10]. Alternatively, an isotropic mixing sequence can be applied during the time period of reaction [34]. This has the net effect of changing the average Hamiltonian such that $\mathcal{H} \propto \mathbf{I} \cdot \mathbf{S}$, which commutes with $\rho_{\text {mix }}$ and prevents any evolution. In this way, an ensemble of polarized molecules can be built up and then released at the same instant to evolve together in phase.

\subsubsection{ALTADENA}

An alternative to performing the hydrogenation within the bore of the magnet is to hydrogenate the substrate in the Earth's magnetic field, or the fringe field of the main magnet, and then transport the sample into the NMR probe within the magnet bore. This is ALTADENA, adiabatic longitudinal transport and dissociation engenders nuclear 
alignment [127].

Because of the very small magnetic field, the H's are still strongly coupled after hydrogenation, causing the H's to remain in the singlet state. If the sample is then transported to the main field by dropping the sample tube down the magnet bore, or flowing the product into the magnet bore, the result is a continuous ramping up of the magnetic field. Mathematically, this is well described by adiabatic transport [115], i.e., the Hamiltonian is smoothly transformed from $\mathcal{H}_{\mathrm{LF}}$ to $\mathcal{H}_{\mathrm{HF}}$ and the overall quantum state must conserve its respective projections onto the instantaneous eigenstates of the transforming Hamiltonian. One can show $[10,127]$ that as the field is adiabatically increased, $\left|S_{0}\right\rangle$ and $\left|T_{0}\right\rangle$ transform into $|\alpha \beta\rangle$ and $|\beta \alpha\rangle$ (or vice versa, depending on the sign of $\left.\left(\omega_{I}-\omega_{S}\right) / 2 \pi J\right)$. This implies that the spin populations in the state $\left|S_{0}\right\rangle\left(\left|T_{0}\right\rangle\right)$ will be carried over into state $|\alpha \beta\rangle(|\beta \alpha\rangle)$. This is depicted in fig. 1.10 and results in a spectrum composed of in-phase absorptive and emissive peaks.

Product operators are needed to calculate the dependence of the spectrum on parameters, such as flip angle, but the characteristic combination of absorptive and emissive peaks is a hallmark of ALTADENA $[9,36]$. From a product operator perspective, $\rho_{\text {mix }}$ is initially an eigenstate of the low field Hamiltonian. Due to the adiabatic transport to high field, the resulting Hamiltonian is $\mathcal{H}=\frac{\mathbf{1}_{4}}{4}+\frac{1}{2}\left( \pm \mathbf{I}_{z} \mp \mathbf{S}_{z}-2 \mathbf{I}_{z} \mathbf{S}_{z}\right)$.

For the adiabatic condition to be strictly true, the Hamiltonian must change at a rate that is significantly slower than the timescale of the fastest motion generated by the Hamiltonian. The characteristic time scale for NMR is the Larmor precession, $T=\omega_{0}^{-1}=$ 


\section{ALTADENA}

A

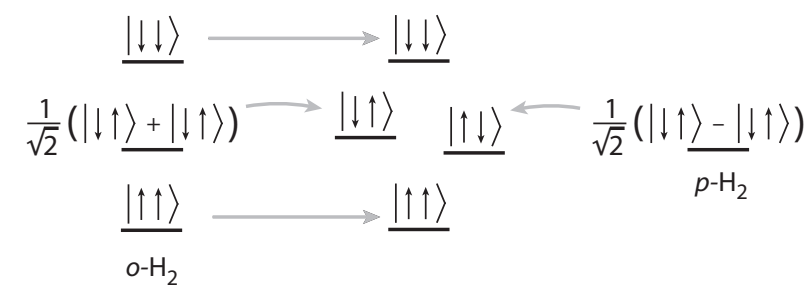

B

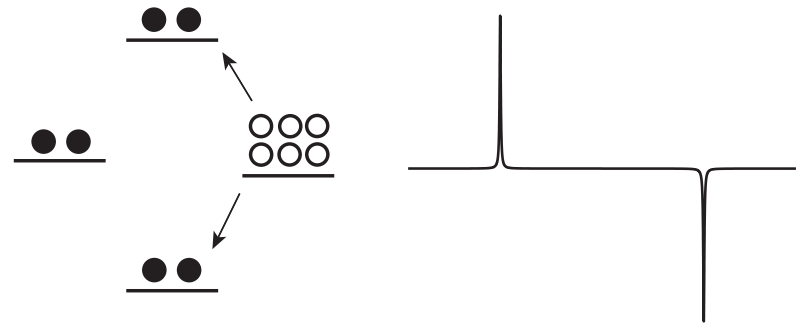

Figure 1.10: The ALTADENA experiment. The $p-\mathrm{H}_{2}$ derived H's remain in a singlet state after hydrogenation. (A) Adiabatic transport results in every eigenstate mapping to a single high field eigenstate. (B) The expected spectrum consists of in-phase absorptive and emissive peaks.

$\left(\gamma B_{0}\right)^{-1}$, and the adiabatic condition requires that

$$
\begin{aligned}
\left|\frac{\mathrm{d} T}{\mathrm{~d} t}\right| & \ll 1 \\
\frac{1}{\omega_{0} B_{0}} \frac{\mathrm{d} B_{0}}{\mathrm{~d} t} & \ll 1 \\
\frac{\vec{G} \cdot \vec{v}}{B_{0} \omega_{0}} & \ll 1 \\
|\vec{v}| & \ll \frac{\omega_{0} B_{0}}{|\vec{G}|}
\end{aligned}
$$

where $\vec{v}$ is the velocity of the gas travelling through the bore of the magnet with a magnetic field gradient $\vec{G}$. A $300 \mathrm{MHz}$ magnet will drop approximately 7 T over less than a meter in the distance between the sweet spot and the outer edge of the dewar. This gives us an estimate of the magnitude of gradient, $|G|<O\left(100 \mathrm{Tm}^{-1}\right)$, and requires that $|\vec{v}| \ll 20 \times 10^{6} \mathrm{~m} / \mathrm{s}$. 
If we separate the time scales by two orders of magnitude to ensure that the adiabatic condition is met, i.e. $\frac{\mathrm{d} T}{\mathrm{~d} t}<0.01$, the gas would need to travel faster than the speed of sound to violate adiabaticity.

\subsubsection{Sensitivity Enhancement}

After hydrogenation with a mixture of $o-\mathrm{H}_{2}$ and $p-\mathrm{H}_{2}$, the nuclear spin system

has a polarization $p=\alpha\left(\frac{1-4 f}{3}\right)$, where the exact proportionality, $\alpha$, depends on the details of the excitation (selective vs. broadband excitation, flip angle, etc.). The SNR benefit of hyperpolarization techniques is typically cast in terms of the signal enhancement, $E$, which is simply the ratio of the hyperpolarized signal strength to the corresponding thermal signal. Because $M_{0} \propto p=\frac{\hbar \gamma B_{0}}{2 T}$ at thermal equilibrium, the enhancement is simply the ratio of the polarization terms:

$$
E=\frac{\alpha \frac{1-4 f}{3}}{\frac{\hbar \gamma B_{0}}{2 T}}=\frac{2 T \alpha(1-4 f)}{3 \hbar \gamma B_{0}} \propto \frac{1-4 f}{B_{0}}
$$

The fact that the enhancement is evaluated with respect to the thermal equilibrium signal strength must be stressed. The polarization derived from the $\mathrm{H}_{2}$ gas is independent of field, but the reference signal is $\propto B_{0}[10,11,28]$. For example, signal enhancement relative to a 10T field can be about $3 \times 10^{4}$, while it will be much bigger relative to the Earth's field, $\approx 6 \times 10^{9}$. Care must be taken when discussing signal enhancement to ensure that potential differences in the reference states are taken into account.

Figure 1.11 shows the dependence of $p-\mathrm{H}_{2}$ induced polarization on the fraction of $p-\mathrm{H}_{2}$. Note that enhancing the fraction of $o-\mathrm{H}_{2}$ also creates a polarized state [17]. Furthermore, the bulk of the polarization enhancement occurs within just a few $\%$ enhance- 
Sensitivity enhancement vs. $\% p-\mathrm{H}_{2}$

A

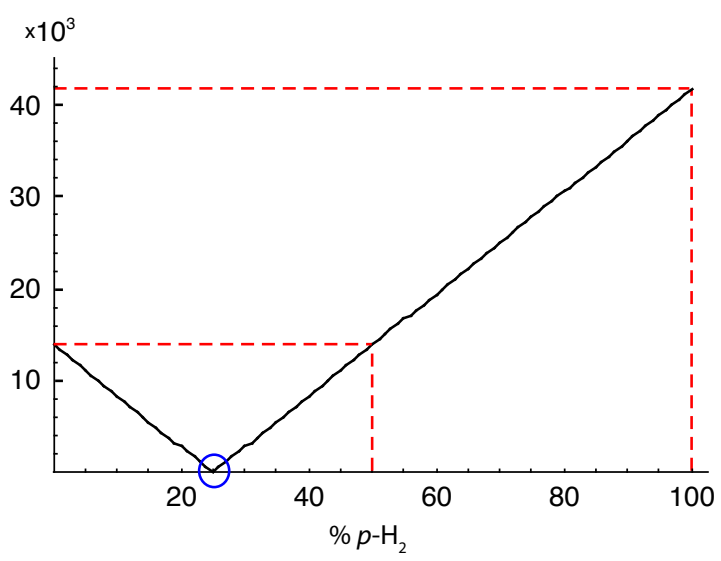

B $\quad \times 10^{3}$

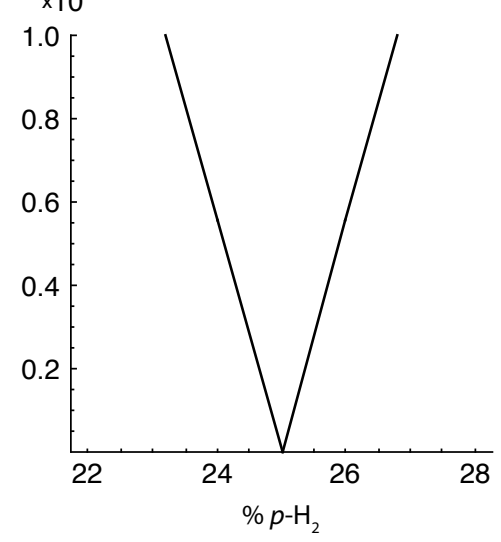

Figure 1.11: (A) Theoretical signal enhancement relative to thermal polarization at 300K and $7.0 \mathrm{~T}$ as a function of $p-\mathrm{H}_{2}$ fraction. (B) Detail of enhancement near the thermal equilibrium value of $25 \% p-\mathrm{H}_{2}$. Note that changing the $p-\mathrm{H}_{2}$ fraction only a few percent from $n-\mathrm{H}_{2}$ results in an enhancement of three orders of magnitude, whereas increasing the fraction of $p-\mathrm{H}_{2}$ from $50 \%$ to $100 \%$ only provides a factor of 3 enhancement of sensitivity.

ment of the $p-\mathrm{H}_{2}$ fraction. If the deviation of $f$ from $25 \%$ is given as $0 \leq n \leq 3 / 4$ then the resulting polarization is $\sim \frac{4}{3} n$, to be compared with a thermal polarization of $\sim 2 \times 10^{-5}$. Increasing (or decreasing) the fraction of $p-\mathrm{H}_{2}$ only a few percent (i.e. $n \approx 10^{-2}$ ) results in three orders of magnitude enhancement of the signal. As mentioned above, the simplest way to increase the fraction of $p-\mathrm{H}_{2}$ is to use liquid $\mathrm{N}_{2}$, which results in $\approx 50 \%$ and a resulting enhancement of $\approx 3 \times 10^{4}$ relative to $300 \mathrm{~K}$ and $7.0 \mathrm{~T}$, whereas $100 \% p-\mathrm{H}_{2}$ only increases this fraction to $\approx 5 \times 10^{4}$. This is why most applications of parahydrogen-induced polarization use the much simpler approach of liquid $\mathrm{N}_{2}$ instead of the more complicated equipment needed for lower temperatures. 


\subsection{Applications}

The use of $p-\mathrm{H}_{2}$ induced polarization has grown dramatically over the past two decades. This development of $p-\mathrm{H}_{2}$ polarization from an interesting novelty to a relatively routine technique is documented in a number of excellent reviews $[26,36,44,56,61,96,118]$. A full review of the literature is not attempted here, but I would like to point out the most active areas of research and the more recent articles not covered by these reviews.

The most common use of $p-\mathrm{H}_{2}$ polarization is in the study of homogeneous catalysis $[4,14,26,27,29,58,64,66,85,96,103,104,120,161]$. The large signal enhancement allows one to observe intermediate species and their rearrangements and to monitor reaction kinetics. The fastest growing application of $p-\mathrm{H}_{2}$ polarization is in the medical imaging community. Specifically, a variety of applications ranging from MRI contrast agents $[7,23,59,67-70,86,87]$ to metabolomic studies $[155,158]$ are performed by transferring the large ${ }^{1} \mathrm{H}$ polarization to longer lived heteronuclei like ${ }^{13} \mathrm{C}$. There has also been interest in using $p-\mathrm{H}_{2}$ to generate entangled quantum states of very high purity for studies in quantum information processing $[10,11,28]$. This active interest in $p-\mathrm{H}_{2}$ polarization has also prompted a number of theoretical studies investigating the mechanism of ortho and para interconversion in a variety of chemically relevant conditions $[8,13,110,151]$.

It is important to note that all of these applications employ homogeneous catalysis to generate the PHIP signal. In the context of in vivo imaging, this poses a problem, as the catalyst must be removed prior to injecting the contrast agent into the patient. It was this problem that let to the fruitful collaboration between Alex Pines' lab and the International Tomography Center with the idea that a cleverly designed heterogeneous catalyst would still 
produce strong polarization as long as the pair-wise mechanism was preserved. Not only were we successful in demonstrating this, but our system turn out to be ideal for producing a stream of polarized gas. This, in turn, has proceeded towards packed-bed micro-reactor imaging. The results presented here are proof of principle and demonstrate only a few examples of the many possible applications of heterogeneous catalysis in the field oh $p-\mathrm{H}_{2}$ induced polarization. It is my hope that others will find interesting applications of these initial ideas and further develop them into routine tools. 


\section{Chapter 2}

\section{Parahydrogen Polarization using}

\section{Heterogeneous Hydrogenation}

The results presented in this chapter have been published previously [91] and are presented here with the permission of the authors.

\section{$2.1 \quad$ Introduction}

The problem of low equilibrium polarization in NMR and MRI has instigated the use of hyperpolarized spin systems (sec. 1.1.3). In particular, it has been demonstrated recently that $p-\mathrm{H}_{2}$ induced polarization can be used to produce liquid solutions with highly polarized ${ }^{13} \mathrm{C}$ nuclei for high quality, single-shot, sub-second angiography of blood vessels of a guinea pig or a rat $[23,70,86]$. However, the fact that the homogeneous catalyst is dissolved in the fluid along with polarized products can be a major obstacle for a much wider practical utilization of the hyperpolarization phenomenon. Catalyst recovery is important in many 
applications where the toxicity and biocompatibility of the catalyst are of prime concern or whenever the catalyst is expensive. This proves difficult in the case of $p-\mathrm{H}_{2}$ induced polarization because the lifetime of nuclear spin polarization in the substrate molecule is on the order of several seconds. Bhattacharya et al. have stated, in their angiographic studies [23], that it is possible to rapidly remove charged homogeneous catalysts from a polarized solution, yet this approach cannot be applied to all kinds of catalysts. Another viable approach is to use a heterogenized catalyst instead. Such a catalyst can be supported on a porous surface, on a nanocrystal, a microbead, or held between frits. One can then envisage a reaction scheme in which the reactants make sufficient contact with the catalyst and react to form the polarized product, which is then physically transported to the NMR detection (or imaging) coil, while the catalyst remains fixed in position and is easily recoverable, still active for the next batch of reactants.

To the best of our knowledge, there are no previous reports of observing PASADENA or ALTADENA in a heterogeneous hydrogenation reaction. One study uses the PASADENA effect to probe a solid $\mathrm{ZnO}$ surface [47], but no hydrogenation of a substrate is performed, and the study investigates adsorption dynamics. Another study was published that used colloidal catalyst particles [60], where it was conjectured that heterogeneous catalysts are not expected to give rise to the PASADENA effect. Indeed, industrial hydrogenation and related manufacturing processes often use heterogeneous catalysts comprising highly dispersed $\mathrm{Pt}$ or $\mathrm{Pd}$ metal particles supported on an appropriate porous material such as alumina. Hydrogenation on such catalysts is carried out at elevated temperatures and pressures and involves dissociative chemisorption of hydrogen molecules onto the metal 
particles, migration of hydrogen atoms over the metal particle surface, and spillover onto the support. Under such circumstances, the chances that the quantum correlation of the nuclear spins of the initial $p-\mathrm{H}_{2}$ molecule will be retained and that the two hydrogen atoms of the molecule will end up in the same product molecule are indeed very small [36]. It appeared, therefore, that observation of PASADENA was limited to homogeneous catalysis only.

One of the growing trends in modern catalysis is an attempt to combine advantages of homogeneous catalytic processes with those of heterogeneous catalysis. This can be achieved, for example, by immobilizing a (modified) homogeneous catalyst on an appropriate porous support $[24,52]$. To date, numerous immobilization strategies have been developed, based on hydrogen bonding, ion pairing, chemical tethering to organic or inorganic supports, etc. Many of these approaches have also been applied to hydrogenation catalysts $[16,25,84,113,114,137]$, yielding stable, efficient, and selective catalysts for heterogeneous hydrogenation under mild conditions. The immobilization should, ideally, preserve the chemical characteristics of the catalyst, implying that the reaction mechanism remains unchanged, but a direct proof of this is difficult to furnish. In this paper, we observe, for the first time, PASADENA and ALTADENA spin polarization patterns in hydrogenated product molecules using several heterogenized catalysts, demonstrating that immobilized homogeneous catalysts retain their mechanism of pair-wise molecular addition, preserve the original spin correlation between the protons of the $p-\mathrm{H}_{2}$ molecule, and are therefore suitable for producing polarized fluids without the concomitant problems of dissolved homogeneous catalysts. 


\section{$2.2 \quad$ Experimental Methods}

\subsubsection{Supported Catalysts}

In the present study, we used three kinds of catalysts: Wilkinson's catalyst (1) supported on styrene-divinylbenzene copolymer (hereafter referred to as $1 /$ polymer); 1 supported on modified silica gel, $\mathrm{RhCl}\left(\mathrm{PPh}_{3}\right)_{2} \mathrm{PPh}_{2}\left(\mathrm{CH}_{2}\right)_{2}-\mathrm{SiO}_{2}\left(\mathbf{1} / \mathrm{SiO}_{2}\right)$; and a supported tridentate complex $\mathrm{Rh}(\mathrm{cod})($ sulfos $)-\mathrm{SiO}_{2}\left(\mathbf{2} / \mathrm{SiO}_{2} ; \operatorname{cod}=\right.$ cycloocta-1,5-diene; sulfos $\left.=-\mathrm{O}_{3} \mathrm{~S}\left(\mathrm{C}_{6} \mathrm{H}_{4}\right) \mathrm{CH}_{2} \mathrm{C}\left(\mathrm{CH}_{2} \mathrm{PPh}_{2}\right)_{3}\right)$. The $\mathbf{1} /$ polymer catalyst was obtained from STREM Chemicals Inc. (catalog number: 45-0670, 5g, maroon beads, 20-60 mesh, 20\% crosslinked). The $1 / \mathrm{SiO}_{2}$ was synthesized according to the procedure described elsewhere [136], using commercial Wilkinson's catalyst (Sigma-Aldrich, product \# 199982, 1g) and 2-diphenylphosphinoethyl-functionalized silica gel (Sigma-Aldrich, product \# 538019, 5g, extent of labeling: $0.7 \mathrm{mmol} / \mathrm{g}$ loading, $200-400 \mathrm{mesh})$ as the starting materials. The $\mathrm{RhCl}\left(\mathrm{PPh}_{3}\right)_{3}$ catalyst is known to undergo rapid ligand exchange [39], and therefore the addition of an approximately stoichiometric amount of 2-diphenylphosphinoethyl-functionalized silica gel to a toluene solution of Wilkinson's catalyst leads to formation of the required catalyst [136]. To ensure full replacement of ligands, the mixture was stirred overnight at room temperature. The supported catalyst was then removed from the solution by filtering, washed three times with toluene, and dried under vacuum at RT. To prevent oxidation of the catalyst, all manipulations were carried out under nitrogen atmosphere [84]. The catalyst $\mathbf{2} / \mathrm{SiO}_{2}$ (0.54wt \% Rh) was obtained from P. Barbaro and colleagues [15]. 


\subsubsection{Parahydrogen Production}

Approximately $5 \mathrm{~g}$ of $\mathrm{FeO}(\mathrm{OH})$ (Sigma-Aldrich, 30-50 mesh, used as received) was placed in the straight section of a heat exchanger, which was then immersed in a liquid nitrogen dewar (fig. 2.1A). Ultrahigh purity $\mathrm{H}_{2}$ was first passed through the coiled section of the exchanger and, only thereafter, came in contact with the catalyst. This produces a constant flow of approximately $50 \% p-\mathrm{H}_{2}$. We verify this using porous alumina $\left(-\mathrm{Al}_{2} \mathrm{O}_{3}\right)$ that had been dried for $1 \mathrm{~h}$ at $250^{\circ} \mathrm{C}$ under vacuum. Either normal or para-enriched $\mathrm{H}_{2}$ gas was freely flowing through the cell filled with alumina beads while NMR spectra were acquired. A 2-pulse spin echo sequence with $1 \mathrm{~ms}$ echo time was used to diminish the contribution of the residual water to the detected NMR signal. The background signal was measured after purging alumina with nitrogen gas and was later subtracted from the signal intensity measured in the presence of $\mathrm{H}_{2}$. The decrease in signal relative to the normal $\mathrm{H}_{2}$ gives a quantitative measure of the conversion to $p-\mathrm{H}_{2}$. We observed that we could achieve the desired 1:1 para/ortho ratio at flow rates up to 150sccm. See sec. A.1 for further details.

\subsubsection{Experimental Setup}

Our experiments were performed on a $300 \mathrm{MHz}$ Bruker Avance DRX spectrometer equipped with a $10 \mathrm{~mm}$ BBO (broad-band observe) probe. A variable-temperature unit was used to keep the samples at 65 or $80^{\circ} \mathrm{C}$ to increase the rate of the reactions. All spectra were acquired using $45^{\circ} \mathrm{RF}$ pulses to obtain maximum signal [37] from the PASADENA experiments. Our flow setup comprises five fused silica capillaries $(345 \mu \mathrm{m}$ OD, Polymicro 
Flow setup

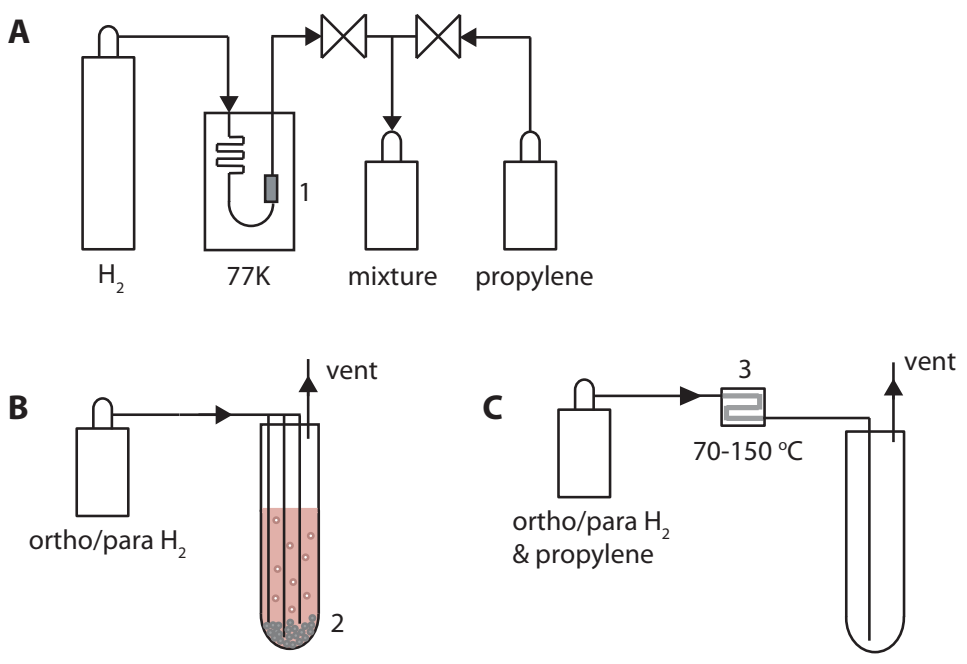

Figure 2.1: (A) Production of para-enriched $\mathrm{H}_{2}$ and preparation of a mixture of gaseous reactants. $n-\mathrm{H}_{2}$ is passed through an ortho/para conversion catalyst $(\mathrm{FeO}(\mathrm{OH})$, labeled as 1) held at $77 \mathrm{~K}$, producing a stream of $50 \% p-\mathrm{H}_{2}$. For gas-phase experiments, para-enriched $\mathrm{H}_{2}$ is stored in a small cylinder (up to 100psi), to which propylene gas is then added. (B) Liquid-phase hydrogenation experiment. The solution is bubbled with $p-\mathrm{H}_{2}$ through an arrangement of $345 \mu \mathrm{m}$ OD capillaries. The bottom of the $10 \mathrm{~mm}$ NMR tube contains one of the supported catalysts (labeled 2), and the solution contains approximately $0.1 \mathrm{M}$ styrene in deuterated benzene. (C) Gas-phase hydrogenation experiment. The mixture of $p-\mathrm{H}_{2}$ and propylene gas flows through a small reactor (labeled 3) consisting of copper tubing packed with a supported catalyst and held at $70-150{ }^{\circ} \mathrm{C}$. The resulting mixture of propane gas with the unreacted propylene and $\mathrm{H}_{2}$ flows into the NMR tube residing in the probe of the NMR spectrometer.

Inc.) that extend to the bottom of the $10 \mathrm{~mm}$ NMR tube to provide the inflow of gas and a $1 / 16$ " tube at the top to vent the gas to a hood (fig. 2.1B). These are attached to a Teflon insert that seals the tube to prevent oxygen from entering and deactivating the catalyst. A pinch valve is located just above the magnet to close both the input and the output lines to allow very quick initiation and termination of the gas flow. Both the pinch valve and the selection of gas are controlled from the spectrometer via TTL lines. Section A.2 contains a complete description of the gas flow setup and its control. 


\subsubsection{Heterogeneous Hydrogenation in Solution}

The samples comprising $4 \mathrm{~mL}$ of $\mathrm{C}_{6} \mathrm{D}_{6}$ and $50 \mu \mathrm{L}$ of styrene were deoxygenated for several minutes by bubbling $\mathrm{N}_{2}$ through the solution before adding the supported catalyst. The $\mathrm{N}_{2}$ flow was left on while assembling the flow setup to purge any oxygen in the NMR tube or other tubing. The prepared sample was placed inside the NMR magnet, and an initial delay of $15 \mathrm{~min}$ was used to allow the reaction mixture to equilibrate at $65^{\circ} \mathrm{C}$. The reaction was initiated by bubbling para-enriched $\mathrm{H}_{2}$ through the solution for some duration of time (ranging from $5 \mathrm{~s}$ to $5 \mathrm{~min}$ ) at a flow rate of $100 \mathrm{sccm}$. After the pinch valve was closed, a delay of $5 \mathrm{~s}$ was used to allow the supported catalyst particles to settle out of the RF-sensitive region before acquisition, protecting against susceptibility-induced broadening in the PASADENA spectra. ALTADENA spectra were obtained by bubbling para-enriched $\mathrm{H}_{2}$ through the sample while holding it outside the magnet, and then quickly placing it in the magnet bore. The $\mathrm{H}_{2}$ used in these experiments came directly from the conversion setup described above, thus being nominally $50 \% p-\mathrm{H}_{2}$.

\subsubsection{Heterogeneous Hydrogenation in Gas Phase}

Each sample was prepared by purging the NMR tube with $\mathrm{N}_{2}$ before adding the supported catalyst and leaving the flow of $\mathrm{N}_{2}$ on while assembling the flow setup. For the PASADENA experiments, the sample was placed inside the NMR magnet, and an initial delay of $10 \mathrm{~min}$ was used for the sample to reach $80^{\circ} \mathrm{C}$. The gaseous reactant mixture was prepared by purging an aluminum cylinder (by alternately filling it with ultrahigh purity $\mathrm{N}_{2}$ and evacuating with a pump) and then filling it with 50psi $\mathrm{H}_{2}$ from the conversion setup 
already described. This cylinder was then filled with an additional 50psi of propylene, yielding a mixture of $50 \%$ propylene, $25 \% p-\mathrm{H}_{2}$, and $25 \% o-\mathrm{H}_{2}$ (fig. 2.1A). PASADENA experiments were performed by completely opening the needle valve regulating the gas flow, and driving the gas at full pressure (20-100psig) to maximize stirring of the catalyst in the NMR tube. The spectrum was acquired while the gas was still flowing through the NMR tube. For the gas-phase ALTADENA experiment, we made a small S-shaped reaction cell from a short (6") length of $1 / 8$ " copper tubing (fig. 2.1C). About $1 \mathrm{~g}$ of the catalyst was packed between plugs of glass wool. The cell was held at $70-150^{\circ} \mathrm{C}$ while the mixture of propylene and $\mathrm{H}_{2}$ flowed through the cell and then into the bore of the magnet to the NMR tube. Typical flow rates ranged between 50 and $225 \mathrm{sccm}$.

\section{$2.3 \quad$ Results}

\subsubsection{Heterogeneous Hydrogenation in Solution}

Both $1 / \mathrm{SiO}_{2}$ and 1 /polymer supported catalysts hydrogenate styrene when $\mathrm{H}_{2}$ is bubbled through the sample tube containing the solution of styrene and the catalyst. More importantly, both catalysts produce clear PASADENA signals as para-enriched $\mathrm{H}_{2}$ is bubbled through the sample at high field. The catalysts also produce ALTADENA signals when para-enriched $\mathrm{H}_{2}$ is bubbled outside the magnet. We also observed that the polymer-supported Wilkinson's catalyst beads appear to be very sensitive to conditions allowing efficient diffusion of the reactants and products into and out of the beads. Long activation times, sometimes greater than $1 \mathrm{~h}$ at elevated temperatures, were needed before the catalyst became active, presumably allowing the beads to swell and expose their catalytic 
centers [72]. Furthermore, having high flow rates of gas that produced good stirring of the beads and long bubbling times (more than 1min) to ensure saturation of the solution with $\mathrm{H}_{2}$ were critical to consistently observe polarization with this catalyst. The $1 / \mathrm{SiO}_{2}$ catalyst, on the other hand, becomes active as soon as the temperature is raised above RT and produces large polarization even for bubbling times as short as a few seconds. The results from the high-field para-enriched hydrogenation (PASADENA) employing these catalysts are shown in fig. $2.2 \mathrm{~A}$ and $\mathrm{B}$, and the ALTADENA spectrum for the $1 / \mathrm{SiO}_{2}$ catalyst is shown in fig. 2.3.

To exclude the possibility that polarization is produced in a homogeneous hydrogenation reaction due to metal complex leaching off the support, the following test was performed. After hydrogenation, the solution was separated from the supported catalyst, and para-enriched $\mathrm{H}_{2}$ was bubbled through the supernatant liquids. The resulting spectra are shown in fig. $2.2 \mathrm{C}$ and $\mathrm{D}$ and demonstrate that the contribution of the homogeneous process is negligible. Additionally, a ${ }^{31} \mathrm{P}$ spectrum detected for this solution is shown in fig. 2.4 (see discussion below).

\subsubsection{Heterogeneous Hydrogenation in Gas Phase}

As a further demonstration that the supported catalysts produce PASADENA signals heterogeneously and not via a homogeneous reaction mechanism, we used $\mathbf{1} / \mathrm{SiO}_{2}$ and $\mathbf{2} / \mathrm{SiO}_{2}$ to para-hydrogenate propylene gas. In the gas phase, there is no possibility for catalyst leaching, and any observation of polarization must stem from a heterogeneous catalytic reaction. Both catalysts were very active in the gas phase and produced exceedingly 

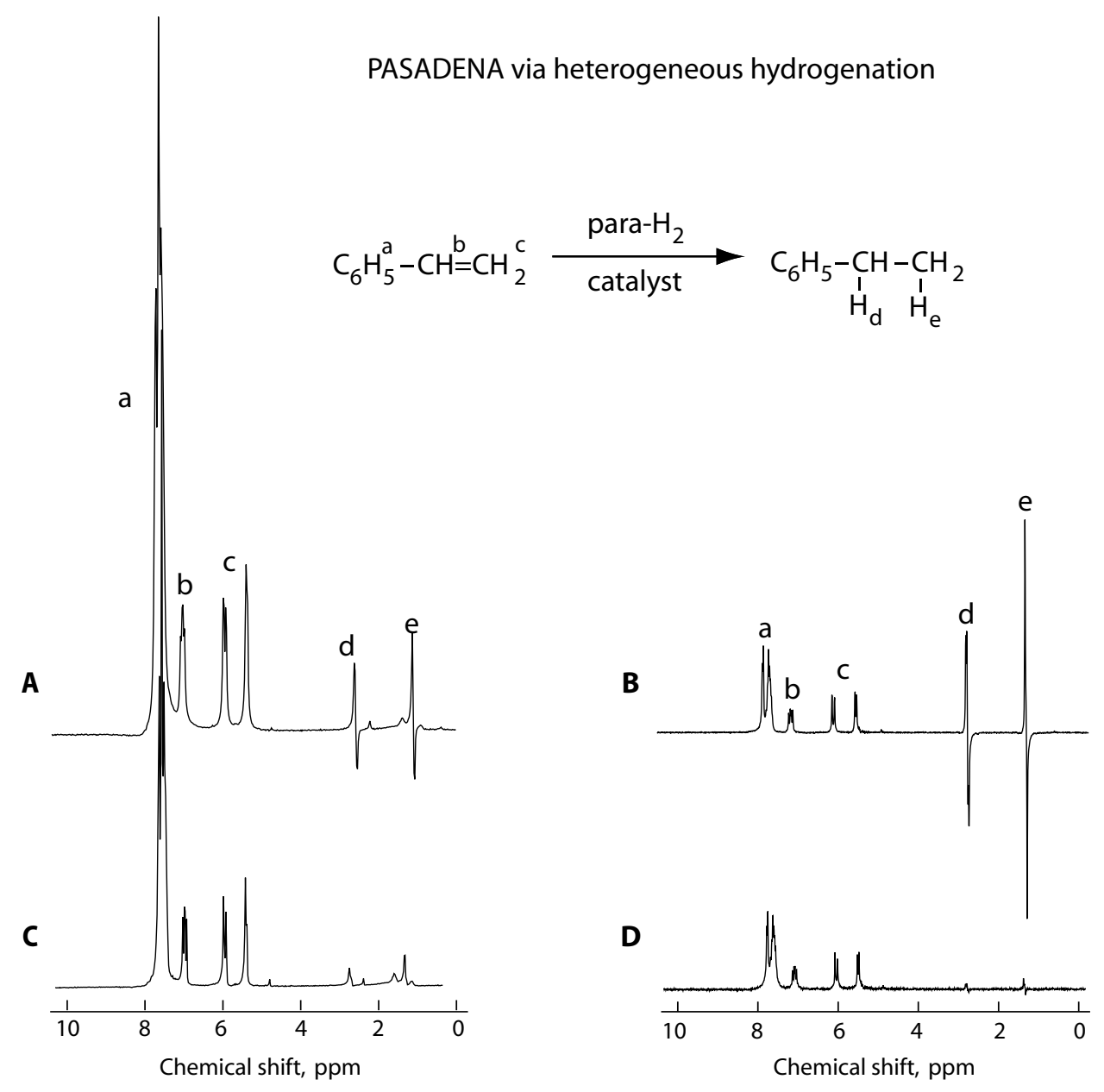

Figure 2.2: Proton NMR spectra for in situ (PASADENA) para-hydrogenation of styrene in $\mathrm{C}_{6} \mathrm{D}_{6}$ at $65^{\circ} \mathrm{C}$. The spectra in $(\mathrm{A})$ and $(\mathrm{C})$ are from $1 / \mathrm{SiO}_{2}$, and those in (B) and (D) are from 1/polymer. Antiphase peaks from the polarized protons in the ethylbenzene product labeled $d$ and $e$ appear at $\delta=2.6$ and $1.2 \mathrm{ppm}$. Spectrum (A) was acquired after para-hydrogenation for $8 \mathrm{~s}$ inside the magnet. Spectrum (B) was acquired after parahydrogenation for $15 \mathrm{~s}$. Spectra (C) and (D) were acquired after bubbling $p-\mathrm{H}_{2}$ gas through the supernatant solutions after removing the catalysts; $(\mathrm{C})$ was acquired after 15min of bubbling, no PASADENA or product formation is observed. The spectrum in (D), acquired after $15 \mathrm{~s}$ of bubbling, shows a very small anti-phase pattern. The spectra $[(\mathrm{A})$ and $(\mathrm{C})][(\mathrm{B})$ and $(\mathrm{D})]$ are plotted to the same vertical scale. 
ALTADENA via heterogeneous hydrogenation

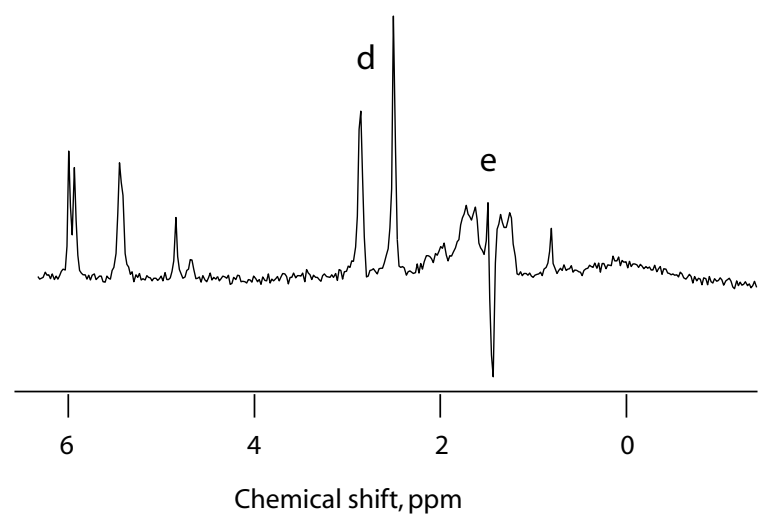

Figure 2.3: Proton NMR spectra from ex situ (ALTADENA) para-hydrogenation of styrene in $\mathrm{C}_{6} \mathrm{D}_{6}$ in the presence of the $\mathbf{1} / \mathrm{SiO}_{2}$ catalyst. The ALTADENA peaks appear as emission and absorption signals at $\delta=1.2$ and $2.6 \mathrm{ppm}$, respectively. The spectrum was acquired with a $45^{\circ}$ flip angle pulse after bubbling $p-\mathrm{H}_{2}$ for $10 \mathrm{~s}$ and subsequent transfer to the high field magnet for detection. 


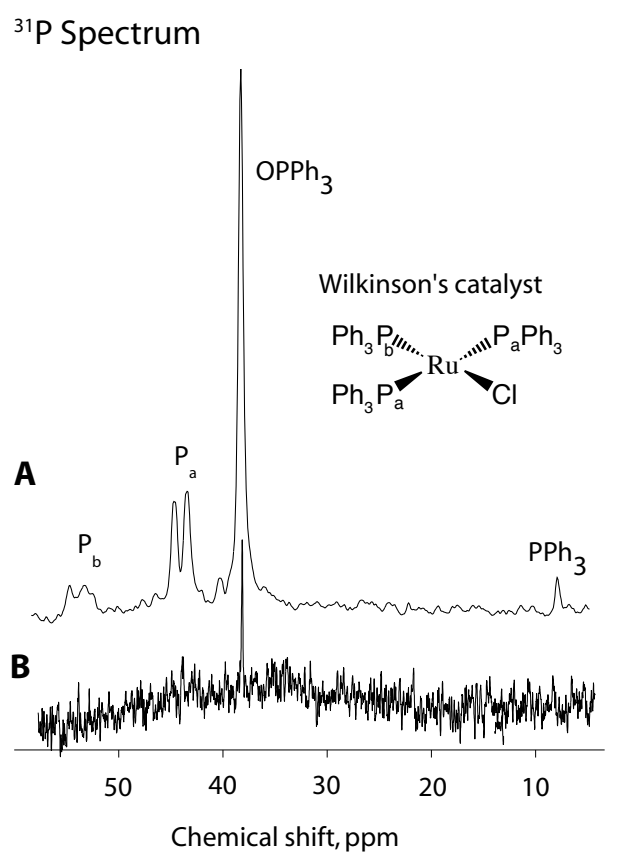

Figure 2.4: ${ }^{31} \mathrm{P}$ NMR spectrum of $(\mathrm{A})$ homogeneous Wilkinson's catalyst dissolved in $\mathrm{C}_{6} \mathrm{D}_{6}$ and (B) supernatant solution recovered after para-hydrogenation of styrene with $\mathbf{1}$ /polymer. The sharp peak around $\delta=38 \mathrm{ppm}$ is assigned to $\mathrm{OPPh}_{3}$. In (A), the multiplets at shifts $\delta=44$ and 54 are from the metal-bound phosphorus nuclei; in (B), neither of these peaks are observed, whereas the broad hump in spectrum (B) indicates the likely presence of very fine heterogeneous polymer particles in solution accounting for the small PASADENA signal in fig. 2.2D. The two spectra were detected using identical experimental parameters except the number of accumulations, which was 512 for spectrum (A) and 4096 for (B). 
strong antiphase multiplets and fast product formation, the results of which are shown in fig. 2.4.

Because of the increased catalytic activity observed with these complexes, we found it important to acquire background spectra with the catalyst removed from the NMR tube before and after each experiment, to ensure the absence of catalytic contaminants from prior hydrogenations as even small amounts can produce enhanced polarizations. We also observed large signal enhancements from ALTADENA experiments with both the $1 / \mathrm{SiO}_{2}$ and the $2 / \mathrm{SiO}_{2}$ catalysts, the spectra being shown in fig. 2.5.

\subsection{Discussion}

Our results constitute the first direct confirmation of the mechanism of the hydrogenation reaction utilizing immobilized metal complexes. The clear PASADENA and ALTADENA spectral patterns confirm the preservation of the longitudinal spin order between the protons derived from the same $p-\mathrm{H}_{2}$ molecule, proving that the addition is, indeed, pair-wise. Moreover, there can be no a priori certainty that an immobilized complex will produce polarization even if the chemical mechanism of the reaction remains essentially the same as in homogeneous solution. Indeed, the transfer of both hydrogen atoms of an $\mathrm{H}_{2}$ molecule to the same product molecule is a necessary condition for the observation of PASADENA or ALTADENA, but is not a sufficient one. In the transient dihydride complex formed upon interaction of $\mathrm{H}_{2}$ with the catalyst, the equivalence of the two $\mathrm{H}$ atoms is lost, and the initial coherence of nuclear spins starts to decay due to spin relaxation processes [42]. Therefore, significant polarization in the product can be expected only if the lifetime of the 
PASADENA via heterogeneous hydrogenation

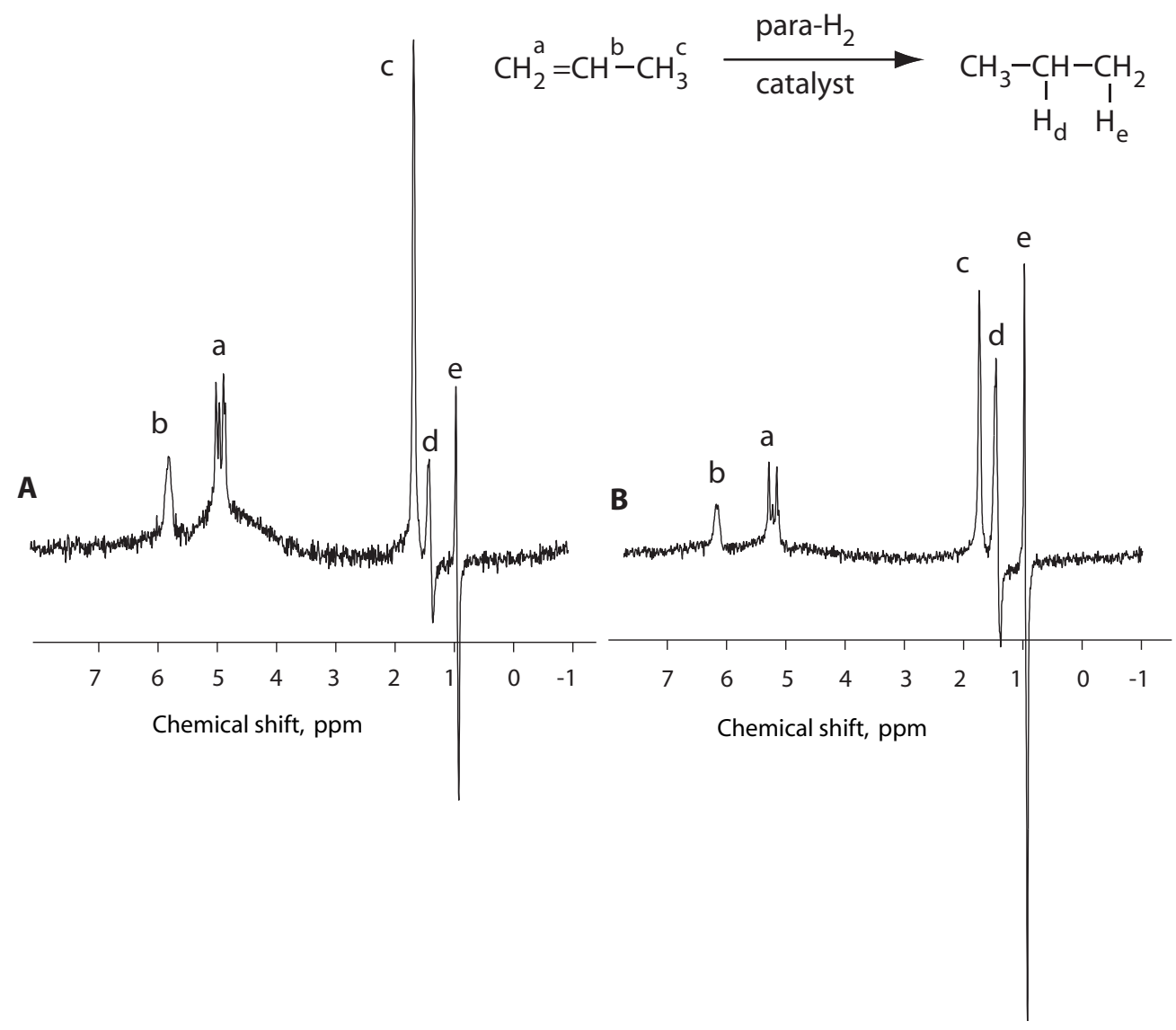

Figure 2.5: Proton NMR spectrum from the in situ hydrogenation of propylene with $50 \%$ $p-\mathrm{H}_{2}$. Spectrum (A) shows the result obtained using the $1 / \mathrm{SiO}_{2}$ catalyst, and (B) is from the $2 / \mathrm{SiO}_{2}$ catalyst. The sample temperature was $80^{\circ} \mathrm{C}$. The peaks from the polarized spins in the propane product appear at $\delta=1.4$ and $0.96 \mathrm{ppm}$ and are labeled as $d$ and $e$ in the spectra. The remaining peaks are from the unreacted propane, and the hump between $\delta=4$ and $5 \mathrm{ppm}$ is from $o-\mathrm{H}_{2}$. Due to the absence of a lock reference, the chemical shift scale is internally calibrated with respect to the propylene $\mathrm{CH}_{3}$ peak. 
intermediate dihydride complex is not much longer than its spin-lattice relaxation time. Because the mobility of the dihydride complex is expected to change substantially upon immobilization, this could lead to a significant enhancement of nuclear relaxation processes and to a complete loss of spin coherence. Furthermore, subtle modifications of the ligands surrounding transition metal complexes can have a pronounced effect on the reaction efficiency and, in particular, the lifetime of the dihydride complex. A more stable dihydride intermediate may still yield hydrogenation product, but could be detrimental for the observation of $p-\mathrm{H}_{2}$-induced polarization. The results presented above demonstrate that there are systems where this does not happen.

With reactions catalyzed by supported complexes, there is always a question of whether the formation of the product and/or polarization is indeed due to heterogeneous catalysis and not catalyzed by rhodium complexes that leach off the surface and are present in solution. To establish a control for this potential problem, we separated the solutions from the catalyst after the hydrogenation experiment and bubbled para-enriched $\mathrm{H}_{2}$ through the supernatant liquids. The resulting spectra shown in fig. $2.2 \mathrm{C}$ and $\mathrm{d}$ demonstrate that the solution from the polymer-supported Wilkinson's catalyst beads gave a very small polarization (with an absolute integrated intensity that is approximately $3 \%$ of the PASADENA signal with the catalyst). Such a dramatic decrease of polarization efficiency rules out the possibility of seeing polarization due solely to catalyst leaching into solution and acting homogeneously. Furthermore, a ${ }^{31} \mathrm{P}$ spectrum of this solution, shown in fig. 2.4, contains no peaks from free Wilkinson's catalyst, but only a sharp peak from the oxidized form of the $\mathrm{PPh}_{3}$ ligand, which dissociates from the $\mathrm{Rh}$ precatalyst [75]. Additionally, a very broad 
peak is also evident, which is consistent with small polymer particles dispersed through the solution. Both the distribution of polymer chain lengths (and thus ${ }^{31} \mathrm{P}$ content) as well as the rigid polymer that the ${ }^{31} \mathrm{P}$ nuclei are attached to would contribute to a very broad ${ }^{31} \mathrm{P}$ peak. Most likely, the small polarization observed in the decanted solution is due to such small polymer particles, some of which have the bound catalyst and give rise to the small PASADENA signal patterns in fig. 2.2D. When testing $1 / \mathrm{SiO}_{2}$, we avoided the potential problem of very fine particles dispersed in solution by filtering the solution through a Hirsch funnel $\left(4-5.5 \mu \mathrm{m}\right.$ pore size). We then bubbled $p-\mathrm{H}_{2}$ through this solution for 15min and observed no detectable PASADENA signal or product buildup, as demonstrated with the spectrum shown in fig. $2.2 \mathrm{C}$. The solution was recombined with the catalyst, and PASADENA signal was again observed (data not shown), demonstrating that the reaction is being catalyzed solely by the heterogenized catalyst. While such tests cannot rule out the presence of traces of the metal catalyst in solution, they do prove that spin polarization is almost exclusively produced by the immobilized catalyst.

Numerous NMR and MRI applications, in particular those carried out in low and ultralow magnetic field environments [111,157], could immensely benefit from the ability to produce polarized media (both liquid and gas phase), free of dissolved catalyst. ${ }^{1}$ For low field measurements, the thermal polarization is exceedingly small, and it is, therefore, very attractive to utilize $p-\mathrm{H}_{2}$-induced polarization for increased sensitivity. Our gasphase ALTADENA experiments demonstrate that it is also possible to hyperpolarize a

\footnotetext{
${ }^{1}$ We note that the procedures outlined above are not suitable for an immediate use in in vivo applications. Such applications would require the use of different solvents and substrates, and a careful measurement of the residual quantities of the metal in solutions after the reaction. While our tests indicate that supernatant solutions are inactive in hydrogenating the substrates and in producing polarization, they cannot exclude the presence of residual amounts of metal in its inactive form.
} 
continuously flowing stream of a fluid by passing it through a catalytic reactor packed with an active heterogenized catalyst. This mechanism can become a promising candidate for replacing the costly ${ }^{3} \mathrm{He}$ or ${ }^{129} \mathrm{Xe}$ gases that are currently used for void-space MRI. Previously demonstrated applications include hyperpolarized lung imaging $[109,124,131]$, or measuring profiles of fluid flow in porous media [73] or micro-channels [76,78]. Heterogenized catalysts can also contribute to the rapidly advancing area of studying catalyzed reactions with NMR micro-fluidic technology $[122,154]$. For example, a porous silicon layer supporting the catalyst and etched in the form of a micro-channel can simulate a catalytic microreactor [105], allowing the possibility of investigating multiphase reactions in the silicon chip. It should also be mentioned that heterogeneously catalyzed para-hydrogenations can, just as their homogeneous counterparts, lead to enhanced signals on other nuclei. For example, the ALTADENA and field cycling methods can efficiently transfer polarization to ${ }^{13} \mathrm{C}$ nuclei, which are especially attractive for their slow longitudinal relaxation rates. It is straightforward to implement these methods using immobilized catalysts and, therefore, to achieve long-lived, polarized nuclear spin states, opening up possibilities for enhanced signal-to-noise imaging and spectroscopy $[9,23,70,86]$.

Last, we would like to stress that conditions of hydrogenation and sample transfer used in this work were not optimized, and yet hydrogenation at a reasonable temperature and atmospheric pressure has yielded pronounced nuclear spin polarization. This gives us confidence that this first observation of $p-\mathrm{H}_{2}$-induced polarization in a heterogeneous reaction can be developed into a useful practical tool for sensitivity-enhanced NMR and MRI. 


\section{Chapter 3}

\section{Gas-Phase MRI using}

\section{Heterogeneous Parahydrogen}

\section{Polarization}

The results in this chapter were published previously [35], and are presented here with the permission of the authors. Also, a brief introduction to the principles of MRI is included for completeness.

\subsection{Magnetic Resonance Imaging}

\subsubsection{Basic Principles}

Section 1.1.2 introduced the Bloch equation for calculating the time evolution of the net magnetization. In the case of a simple excitation followed by free precession in a 
homogeneous field, the resulting signal is

$$
s(t) \propto \int \mathrm{d} V M_{0} \mathrm{e}^{-\imath \omega t} \mathrm{e}^{-t / T_{2}}
$$

The Fourier transform of this signal gives a single, sharp resonance with a line width $\approx \frac{1}{T_{2}}$. The fact that the nuclei are sensitive to the local magnetic field suggests that a spatially inhomogeneous field should provide a means of extracting spatially resolved information from the NMR signal. A detailed introduction to NMR imaging, or MRI, can be found in many texts ranging from medical to materials imaging [32,43,89,121]. As an introduction for the following chapters, it is sufficient to state the key concepts without substantial elaboration.

In addition to the static magnetic field, additional magnetic fields that vary linearly with position (over the desired region) are added. These so-called gradient fields are designed to be turned on and off quite rapidly, resulting in the term pulsed field gradients (pfg),

$$
B(\vec{x}, t)=B_{0}+G_{x}(t) x+G_{y}(t) y+G_{z}(t) z=B_{0}+\vec{G}(t) \cdot \vec{x}
$$

The phase acquired by a given nuclear spin after a time, $t$, is

$$
\begin{aligned}
\phi(\vec{x}, t) & =\gamma \int_{0}^{t} B(\vec{x}, \tau) \mathrm{d} \tau \\
& =\int_{0}^{t}\left(B_{0}+\vec{G}(\tau) \cdot \vec{x}\right) \mathrm{d} \tau \\
& =\omega t+\vec{k}(t) \cdot \vec{x}
\end{aligned}
$$

where the k-vector is defined as

$$
\vec{k}(t)=\gamma \int_{0}^{t} \vec{G}(\tau) \mathrm{d} \tau
$$


The resulting signal from the entire sample is

$$
s(t) \propto \int \mathrm{d} V M_{0}(\vec{x}) \mathrm{e}^{-\imath \phi(\vec{x}, t)}=\int \mathrm{d} \vec{x} M_{0}(\vec{x}) \mathrm{e}^{-\imath \omega t} \mathrm{e}^{-\imath \vec{k}(t) \cdot \vec{x}} \propto \mathcal{F}\left\{M_{0}(\vec{x})\right\}=\tilde{M}_{0}(\vec{k}(t))
$$

The important result here is that the signal equation is proportional to the Fourier transform of the object in the RF coil. Furthermore, the position in k-space being sampled (i.e. the kspace trajectory) is controlled by manipulating the amplitudes of the gradients in time. The basic approach in MRI is to record a series of k-space trajectories. Once a sufficient amount of k-space data has been gathered, it is assembled and Fourier transformed to reconstruct the original image as depicted in fig. 3.1.

A number of complications arise that make things less straightforward than the simple picture presented above. In particular, short relaxation times, resonance offsets due to chemical shift or susceptibility induced field gradients as well as motion of the nuclear spins can all cause significant artifacts in the reconstructed image. The severity and form of the artifacts depends on the specific k-space trajectory.

There are many approaches to dealing with such complications [43,121]. One simple approach to removing many artifacts in the acquired image is to use single point imaging (SPI). The primary benefit of SPI techniques is the constant time encoding period which separates the evolution time from the k-space trajectory. This causes most sources of artifacts to become simple attenuation factors,

$$
\exp \left(-t_{p} / T_{2}^{*}\right) \exp \left(-\imath \gamma \Delta B(\vec{x}) t_{p}\right) \ldots=c,|c|<1
$$

which can often be partially compensated for with appropriate refocusing echoes. Typical SPI trajectories turn on the pfg during an echo to move to a particular position in k-space 
$\mathrm{k}-$ Space traversal and image reconstruction

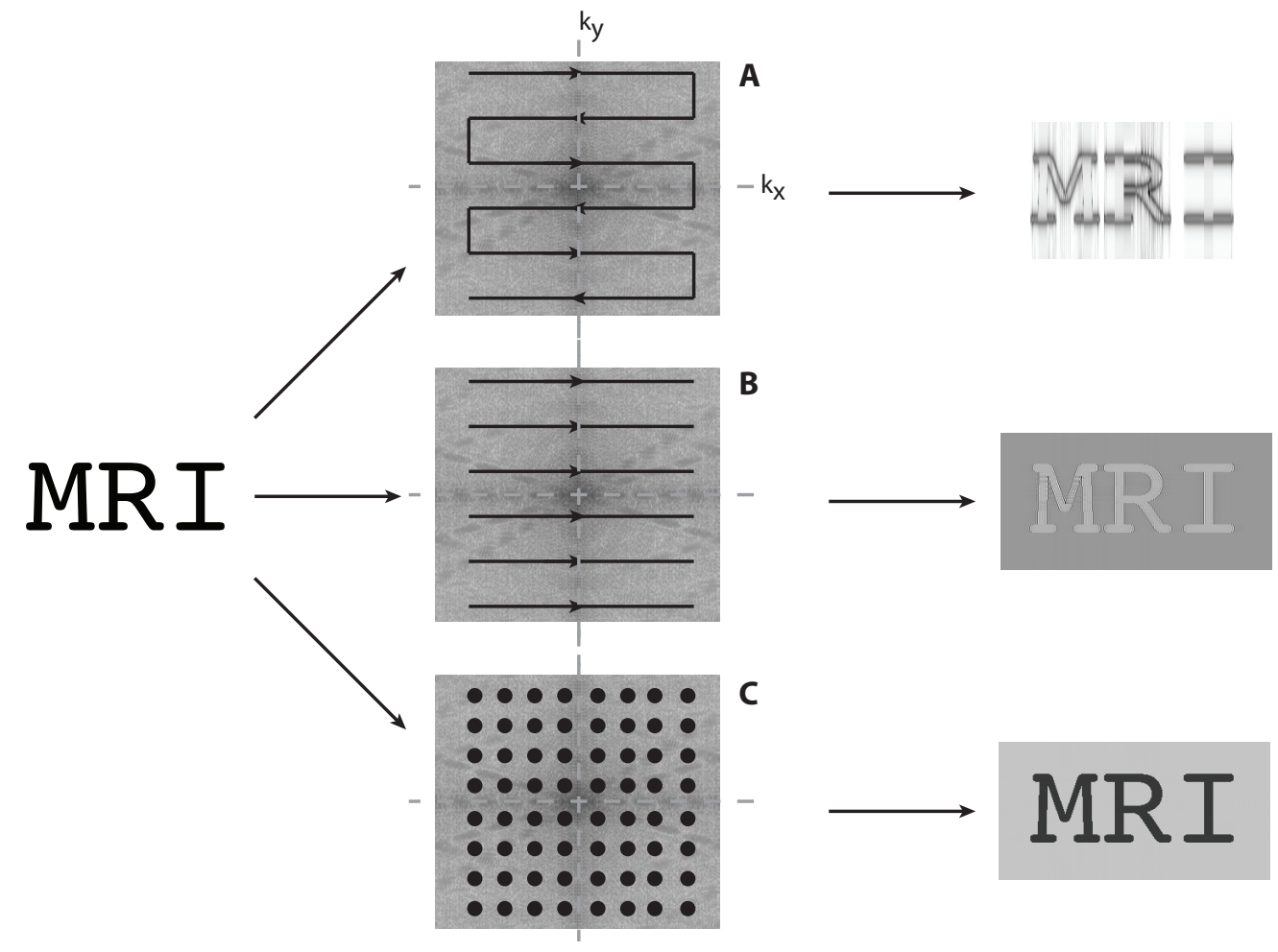

Figure 3.1: Examples of k-space trajectories during MRI acquistion. After sufficient kspace data is acquired, it is Fourier transformed to reconstruct the image. (A) Single shot imaging, acquiring a plane of k-space in one acquisition. (B) Line scan imaging, acquiring lines of k-space in several acquisitions. (C) Single point imaging, acquiring only a single point of k-space with each acquisition. The reconstructed images contain artifacts due to off resonance effects. The exact form of such artifacts depends on the trajectory through $\mathrm{k}$-space. SPI reduces offset effects to a constant phase and attenuation. 
(the phase-encode time, $t_{p}$ ) and then turns off the gradients during the actual signal readout. This has the additional benefit of providing spectroscopic information during the read out interval, known as chemical $s$ hift $i$ maging (CSI). The disadvantage of SPI is the time needed to sample enough of $\mathrm{k}$-space given that it takes an entire scan per point.

The constant encoding time of SPI is not enough to compensate for motion during the encoding interval. Diffusion and bulk flow all lead to phase errors and, thus, artifacts in the image reconstruction [43]. This can actually be advantageous in situations where spatially resolved diffusion or velocity measurements are desired. Section 4.4.6 describes how to use specific pfg profiles to remove phase artifacts due to velocity and acceleration, as well as utilizing those phases to acquire spatially resolved velocity measurements via MRI microscopy.

\subsubsection{MRI of Gases}

A number of scenarios lend themselves to gas-phase MRI studies. Medical imaging of the lungs or sinus cavities; visualizing gas flow through porous materials, such as rock, foams or catalyst beds; and monitoring the spatial profile of reactions involving gases, to name a few. Unfortunately, imaging a gas using thermal polarization is a difficult task. As always, the fundamental problem is low sensitivity. The only difference from the analysis presented in sec. 1.1.3 is the spatial resolution and number of phase-encoding steps, $N_{p}$,

$$
\mathrm{SNR} \propto \rho(\Delta x \Delta y \Delta z) \sqrt{N_{p} N_{s} T_{\text {read }}}
$$

The density of a gas is typically three orders of magnitude smaller than that of a liquid. This requires sacrificing an order of magnitude in resolution in all three dimensions or 
increasing the scan time by a factor of $1,000,000$ to regain the same sensitivity. In cases like micro-imaging where high resolution is the primary goal, the outlook is grim.

These limitations have led to the use of hyperpolarized gases $[40,71,88,109,124,131]$ such as ${ }^{129} \mathrm{Xe},{ }^{3} \mathrm{He}$ and, more recently, ${ }^{83} \mathrm{Kr}$. Other approaches have used fast-relaxing inert fluorinated gases $[95,130]$ to improve SNR per unit time via fast signal averaging or higher gas pressures to directly increase SNR [102]. Drawbacks to these techniques include the fact that hyperpolarized noble gases and polarization instruments are generally expensive; only certain gases have strong enough spin-rotation interaction to have significantly shortened $T_{1}$; and the SNR only scales, at most, linearly with density, which limits the SNR improvement that can reasonably be obtained via increased pressure.

Another method for producing hyperpolarized fluids is by using $p-\mathrm{H}_{2},[36-38,118]$. Section 1.2 discusses how $\mathrm{H}_{2}$ mixtures enriched with one of the spin isomers provide large non-equilibrium nuclear spin order. Section 1.3 explains how this enhanced spin order can be converted into an enhanced NMR signal if the enriched ortho-/para- $\mathrm{H}_{2}$ mixture participates in a hydrogenation reaction that breaks the symmetry of the original $\mathrm{H}_{2}$ molecule. If the hydrogenation reaction preserves the spin correlation between the protons, this correlation can be converted into a strong observable nuclear spin polarization.

The method is not limited to protons only, as polarization transfer mechanisms $[9,26,86]$ can be employed to polarize other nuclei as well, such as a slowly relaxing ${ }^{13} \mathrm{C}$. Contrast agents based on $p-\mathrm{H}_{2}$ polarization have indeed been demonstrated $[23,70,86]$ and provide high-quality, angiographic ${ }^{13} \mathrm{C}$ MR images of the blood vessels in animal models. However, these experiments were conducted in the liquid phase and are not applicable 
for polarizing of gases. We have recently reported [91] the creation and observation of $p-\mathrm{H}_{2}$ polarization in heterogeneous hydrogenation reactions. Of particular interest is the demonstration that it is possible to create $p-\mathrm{H}_{2}$ polarized gases via heterogeneous catalysis.

\subsection{Introduction}

This work represents the first demonstration of signal enhancement in gas-phase imaging employing a supported catalyst. We demonstrate gas-phase proton imaging of the hyperpolarized product resulting from para-hydrogenation of a substrate molecule, in our case, propylene $\left(\mathrm{CH}_{3} \mathrm{CH}=\mathrm{CH}_{2}\right)$. The reaction is catalyzed by heterogenized Wilkinson's catalyst supported on modified silica gel $\left(\left[\mathrm{RhCl}-\left(\mathrm{PPh}_{3}\right)_{2} \mathrm{PPh}_{2}\left(\mathrm{CH}_{2}\right)_{2}\right]-\mathrm{SiO}_{2}\right)$ and results in the formation of propane $\left(\mathrm{CH}_{3} \mathrm{CH}_{2} \mathrm{CH}_{3}\right)$. The $p-\mathrm{H}_{2}$-derived protons in propane (labeled as $\mathrm{H}_{A}$ and $\mathrm{H}_{B}$ in fig. 3.2) preserve their nuclear spin singlet state [10,36] at low magnetic fields. The reactant-product mixture is then adiabatically transferred to the high field of the NMR magnet, leading to preferential population of the $|\alpha \beta\rangle$ (or the $|\beta \alpha\rangle$ ) state, an experimental scheme referred to as ALTADENA [127]. The resulting NMR signal is greatly enhanced compared to what is achievable with thermal population of spin states.

The enhancement is evaluated with respect to the thermal equilibrium state as discussed in sec. 1.3.4. Therefore, even though the $p-\mathrm{H}_{2}$-derived polarization is fieldindependent $[10,11,28]$, the enhancement depends on the field strength of the reference field for the thermal state (assuming constant T). For example, for a 10T field the enhancement can be about $3 \times 10^{4}$, while a standard $1.5 \mathrm{~T}$ medical scanner would exhibit an enhancement of $2 \times 10^{5}$. 
Chemical reactions

A

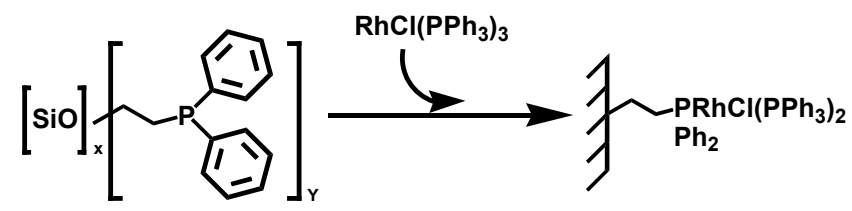

B

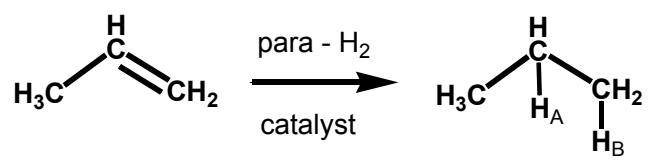

Figure 3.2: (A) Schematic of the preparation of the supported catalyst used in the study. (B) para-hydrogenation of propylene to form propane gas (the protons from $p-\mathrm{H}_{2}$ in propane are labeled $\mathrm{H}_{A}$ and $\mathrm{H}_{B}$ ).

Hydrogenation takes place in the catalytic cell that is placed in the Earth's magnetic field $(0.5 \mathrm{G})$, where the thermal equilibrium spin density matrix is a mixture of the singlet state $\left(\mathbf{S}_{0}\right)$ and equal amounts of each of the three triplets $\left(\mathbf{T}_{0}, \mathbf{T}_{1}\right.$, and $\left.\mathbf{T}_{1}\right)$; these states are in fact the eigenstates of the Earth's field nuclear spin rotating frame Hamiltonian,

$$
\mathcal{H}_{\mathrm{LF}}=2 \pi J\left(\mathbf{I}_{x} \mathbf{S}_{x}+\mathbf{I}_{y} \mathbf{S}_{y}+\mathbf{I}_{z} \mathbf{S}_{z}\right)
$$

where $\mathrm{J}$ is the scalar coupling constant between the protons and I and $\mathrm{S}$ represent the angular momentum spin operators for the two nuclear spins. In the rotating frame, the Zeeman terms are small in the Earth's field and are therefore neglected. This low-field Hamiltonian corresponds to an $\mathrm{A}_{2}$ spin system.

The singlet and triplet eigenstates are defined in sec. 1.3 and denoted $\mathbf{S}_{0}, \mathbf{T}_{0}, \mathbf{T}_{ \pm 1}$. The singlet state is called $p-\mathrm{H}_{2}$ while the triplet states are collectively called $o-\mathrm{H}_{2}$. If the molar fraction of $p-\mathrm{H}_{2}$ in the hydrogen mixture is $f$, the density matrix after hydrogenation will comprise a fraction $f$ of the spin singlet and $1-f$ of a balanced mixture of the three 
spin triplets. This is expressed in product operator terms as

$$
\rho_{\text {low }}=\mathbf{1} / 4+\frac{1-4 f}{3}\left(\mathbf{I}_{x} \mathbf{S}_{x}+\mathbf{I}_{y} \mathbf{S}_{y}+\mathbf{I}_{z} \mathbf{S}_{z}\right)
$$

Clearly, this is a non-equilibrium state. Compared to the thermal equilibrium state

$$
\rho_{e q}=\mathbf{1} / 4+\epsilon\left(\mathbf{I}_{z}+\mathbf{S}_{z}\right)
$$

where $\epsilon \approx h \gamma / k T$, implying enhancement factors of up to about $1 / \epsilon$ (see discussion in sec. 1.3.4.

As propane is adiabatically transferred to the high field of the magnet, the Hamiltonian gradually transforms to its high field form

$$
\mathcal{H}_{\text {high }}=\omega_{I} \mathbf{I}_{z}+\omega_{S} \mathbf{S}_{z}+2 \pi J_{I S} \mathbf{I}_{z} \mathbf{S}_{z}
$$

with $\omega_{I}$ and $\omega_{S}$ corresponding to the chemical shifts of the protons $\mathrm{H}_{A}$ and $\mathrm{H}_{B}$. This highfield Hamiltonian is accurate under the condition that $\left|\omega_{I}-\omega_{S}\right| \gg\left|J_{I S}\right|$ and corresponds to an AX spin system. The eigenstates of $\mathcal{H}_{\text {high }}$ are $|\alpha \alpha\rangle,|\alpha \beta\rangle,|\beta \alpha\rangle$ and $|\beta \beta\rangle$.

According to the quantum adiabatic theorem [115], the overall quantum state must conserve its respective projections onto the instantaneous eigenstates of the transforming Hamiltonian. One can show $[10,127]$ that as the field is adiabatically increased, $\left|S_{0}\right\rangle$ and $\left|T_{0}\right\rangle$ transform into $|\alpha \beta\rangle$ and $|\beta \alpha\rangle$ (or vice versa, depending on the sign of $\left.\left(\omega_{I}\right)-\omega_{S} / J\right)$. This implies that the spin populations in the state $\left|S_{0}\right\rangle\left(\left|T_{0}\right\rangle\right)$ will be carried over into state $|\alpha \beta\rangle(|\beta \alpha\rangle)$. Consequently, in a high field, the spins of $\mathrm{H}_{A}$ and $\mathrm{H}_{B}$ in propane will be in 
the state described by

$$
\begin{aligned}
\rho_{\text {high }} & =f|\alpha \beta\rangle\langle\alpha \beta|+\frac{1-f}{3}(|\beta \alpha\rangle\langle\beta \alpha|+| \alpha \alpha\rangle\langle\alpha \alpha|+| \beta \beta\rangle\langle\beta \beta|) \\
& =\frac{\mathbf{1}}{4}-\frac{(1-4 f)}{6}\left(\mathbf{I}_{z}+\mathbf{S}_{z}+2 \mathbf{I}_{z} \mathbf{S}_{z}\right)
\end{aligned}
$$

(For convenience we assume that $\left|S_{0}\right\rangle$ maps into $|\alpha \beta\rangle$ and that $\left|T_{0}\right\rangle$ maps into $|\beta \alpha\rangle$.) However, the polarization in $\rho_{\text {high }}$ is still non-observable, therefore the state must be rotated with a RF pulse with a certain flip angle, $\theta$. From the rotated state, one can deduce the expected multiplet pattern [10]. In our case, the expected spectrum is proportional to

$$
\sin \theta[(1-\cos \theta),(1+\cos \theta),(-1-\cos \theta),(-1+\cos \theta)]
$$

where each term represents the relative intensity of the individual lines in the multiplets, with the leftmost two representing the first $(I)$ and the rightmost representing the second spin $(S)$. For example, with a $\theta=\pi / 4$ nutation pulse, the expected spectral pattern is proportional to $[0.3,1.7,1.7,0.3]$. This prediction is in agreement with the experimental observation.

\subsection{Experimental Methods}

For our experiments, a hydrogen mixture enriched in the para spin state $(50 \%$ $p-\mathrm{H}_{2}$ ) was produced by passing pure hydrogen gas through a tubular cell that was packed with $\mathrm{FeO}(\mathrm{OH})$ and immersed in a Dewar flask containing liquid $\mathrm{N}_{2}$. The ortho/para ratio was verified by measuring the NMR signal intensities of $\mathrm{H}_{2}$ in a cell packed with porous alumina $\left(\gamma-\mathrm{Al}_{2} \mathrm{O}_{3}\right.$; the method is detailed elsewhere [91]). An aluminum cylinder was filled with the para-enriched $\mathrm{H}_{2}$ to $80 \mathrm{psi}$, and propylene was then added to a total pressure of 
Flow setup

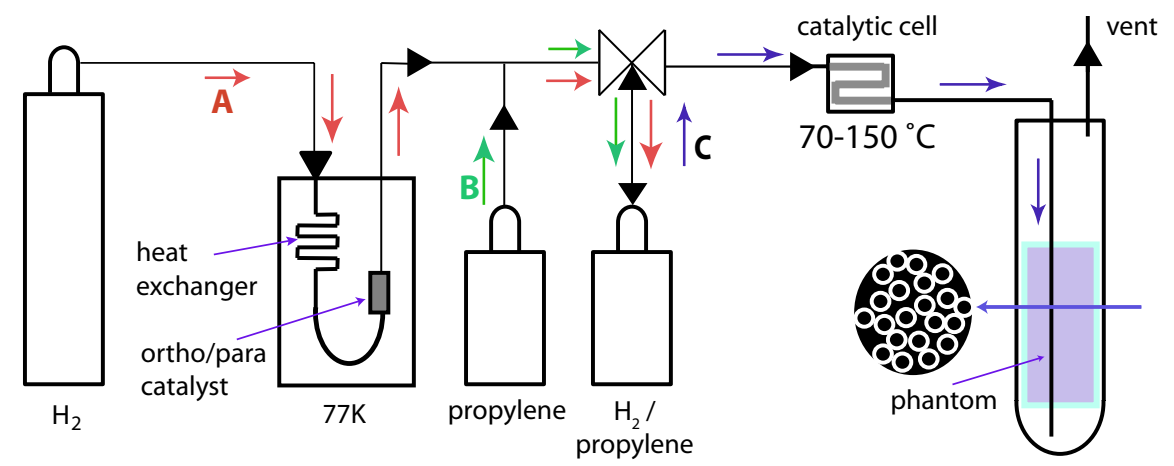

Figure 3.3: Flow diagram for the gas-phase experiment. The red arrows (A) represent the flow of the $\mathrm{H}_{2}$ gas, the green arrows (B) represent the flow of propylene, and the blue arrows (C) depict the flow of the $\mathrm{H}_{2}$ and propylene mixture. Normal $\mathrm{H}_{2}(25 \%$ para) flows through an o/p catalyst, $\mathrm{FeO}(\mathrm{OH})$, held at $77 \mathrm{~K}$ to produce a stream of $50 \% p-\mathrm{H}_{2}$, which is then stored in the small cylinder, to which propylene is added. This experiment used a sample of $40 \%$ ortho- $\mathrm{H}_{2}, 40 \% p-\mathrm{H}_{2}$, and $20 \%$ propylene with a total pressure of 100 psi. A mixture of propylene and para-enriched $\mathrm{H}_{2}$ flows into a catalytic cell containing catalyst supported on silica gel and held at $70-150^{\circ} \mathrm{C}$. The product, propane, as well as unreacted propylene and $\mathrm{H}_{2}$ flow into the magnet, resulting in ALTADENA polarization of the propane. The gas flows through a 1/16" capillary to the bottom of the NMR tube and up through a phantom, either a cross shape or a random packing of $1 / 16$ " capillaries. A cross section of the latter is shown.

100psi (fig. 3.3). Consequently, the volumetric composition in the reactant mixture was $20 \%$ propylene, $40 \% p-\mathrm{H}_{2}$, and $40 \% o-\mathrm{H}_{2}$.

The supported catalyst was prepared $[39,91,136]$ by the addition of an approximately stoichiometric amount of 2-diphenylphosphinoethyl-functionalized silica gel to a solution of Wilkinson's catalyst in toluene (fig. 3.2). The solution was stirred overnight, and the catalyst was filtered, washed with toluene, and dried under vacuum at room temperature. All the chemicals used in the synthesis were obtained from Sigma-Aldrich and used as received. All manipulations with the catalyst were performed under nitrogen gas atmosphere. 
A sample of the catalyst $(\approx 0.1 \mathrm{~g})$ was packed between plugs of glass wool inside a 6 " long section of copper tubing $(d=1 / 8$ "), bent into an S-shaped catalytic reactor. The reactor (polarizer) was held at a temperature of approximately $150^{\circ} \mathrm{C}$. The gas mixture flowed through the catalytic cell and then through Teflon tubing $(d=1 / 8$ "), which finally transitioned into a Teflon capillary $(d=1 / 16 ")$ ending at the bottom of a $10 \mathrm{~mm}$ NMR tube. The tube was placed inside the high field of a vertical bore, $300 \mathrm{MHz}$ NMR magnet (Varian Inc.) equipped with imaging gradient coils. The phantoms were placed near the bottom of the NMR tube and held in the sensitive region of the detector.

\subsection{Results}

The ${ }^{1} \mathrm{H}$ NMR spectra are shown in fig. 3.4. Figure $3.4 \mathrm{~B}$ shows the spectrum of propylene acquired under flowing conditions and before any reaction was performed. The reaction was then carried out by heating the catalytic cell. The emissive and absorptive ALTADENA peaks and the smaller peaks from the unreacted propylene appear in the resulting spectrum (fig. 3.4C). This latter spectrum was also acquired under flow conditions. The propylene peaks correspond to partially relaxed thermal polarization and are much smaller compared to the ALTADENA peaks and serve as an internal intensity reference. In another experiment, the catalytic cell was maintained at high temperature, the gaseous mixture of reactants and product was transferred to the NMR tube, and the flow was stopped. Figure 3.4A shows the resulting spectrum acquired two minutes after stopping the flow. Owing to the long residence time in the NMR tube, complete thermal equilibration can be assumed; the spectrum illustrates the maximum amount of obtainable thermal polarization. 
Furthermore, by comparing the signal intensity per proton between propane (product) and propylene (reactant), we estimate the reaction yield to be about $5 \%$. The spectra acquired in the flowing and stopped-flow conditions are different, reflecting the different pressures and the extent of thermal equilibration achieved during the transit and residence times in the magnet.

Images of gas flowing in the void space surrounding the phantom were acquired using CSI [43]. The pulse sequence consisted of a $45^{\circ} \mathrm{RF}$ nutation pulse, tipping $\rho_{\text {high }}$ into the observation plane $[63,140]$. The detectable terms were then spatially encoded by a $200 \mu$ s phase-encoding gradient pulse. The free induction decay was subsequently acquired in the absence of any gradient. This sequence was repeated for $21 \times 21$ steps of the $x$ and $y$ phase-encoding gradients. The maximum phase-encoding gradient pulse amplitude was $70 \mathrm{G} / \mathrm{cm}$. The frequency components of interest were then selected for the generation of the image. The field of view was $12 \mathrm{~mm} \times 12 \mathrm{~mm}$, which resulted in an in-plane spatial resolution of $0.57 \mathrm{~mm} \times 0.57 \mathrm{~mm}$. The thickness of the image plane (along the $z$ direction) was $10 \mathrm{~mm}$.

The signal from one of the ALTADENA peaks was integrated to generate the ALTADENA image shown in fig. 3.5C and E. The signal-to-noise ratio, as measured directly from the magnitude image (average signal in the gas space divided by the standard deviation of noise located at the edges of the field of view), was 150 in the channels of the phantom with the weaker signal and 200 in the channel exhibiting the strongest signal. In fig. 3.5C, no signal is seen in the $1 / 16$ inch capillary because of the higher flow velocity in it. The strongest signal is seen in the channel opposite to the capillary because a higher fraction of 
the incident gas is deflected by the round bottom of the glass NMR tube into the opposite channel. The total scan time was $8 \mathrm{~min}$, owing to the long recycle delay of $1 \mathrm{~s}$, but could be optimized.

The geometry can be compared to the conventional MRI image, where the void space surrounding the phantom was filled with water (fig. 3.5A). Also shown in fig. 3.5B and D is the thermally polarized signal obtained by integrating the $\mathrm{CH}_{2}$ peaks of the unreacted propylene (also marked in fig. 3.4B). The resulting image exhibits almost no signal except for a slight zero-frequency artifact. This means that compared to the ALTADENA peaks, the thermal polarization is exceedingly low. As a second phantom, we used a random packing of capillaries mimicking a porous medium. The images reconstructed from the unpolarized propylene and ALTADENA-polarized propane peaks are shown in fig. 3.5D and E.

\subsection{Discussion}

The exact amount of polarization enhancement compared to other proton resonances is difficult to obtain directly from these images because of the poor signal in fig. 3.5B and D. The ALTADENA signal yields image signal-to-noise ratios of 150-200. From the data of fig. 3.4A, we estimate the reaction yield to be $5 \%$. The enhancement factor is obtained from the reaction yield and knowledge of the amount of thermal repolarization that is found by comparing the total signal per proton between the stopped-flow and flowing spectra in fig. 3.4A and B. We estimate that during flowing conditions, the propylene peaks are polarized to only about $50 \%$ of their thermal equilibrium value. From the data in fig. $3.4 \mathrm{C}$, the ratio of peak areas for ALTADENA-enhanced propane protons to thermally polarized 


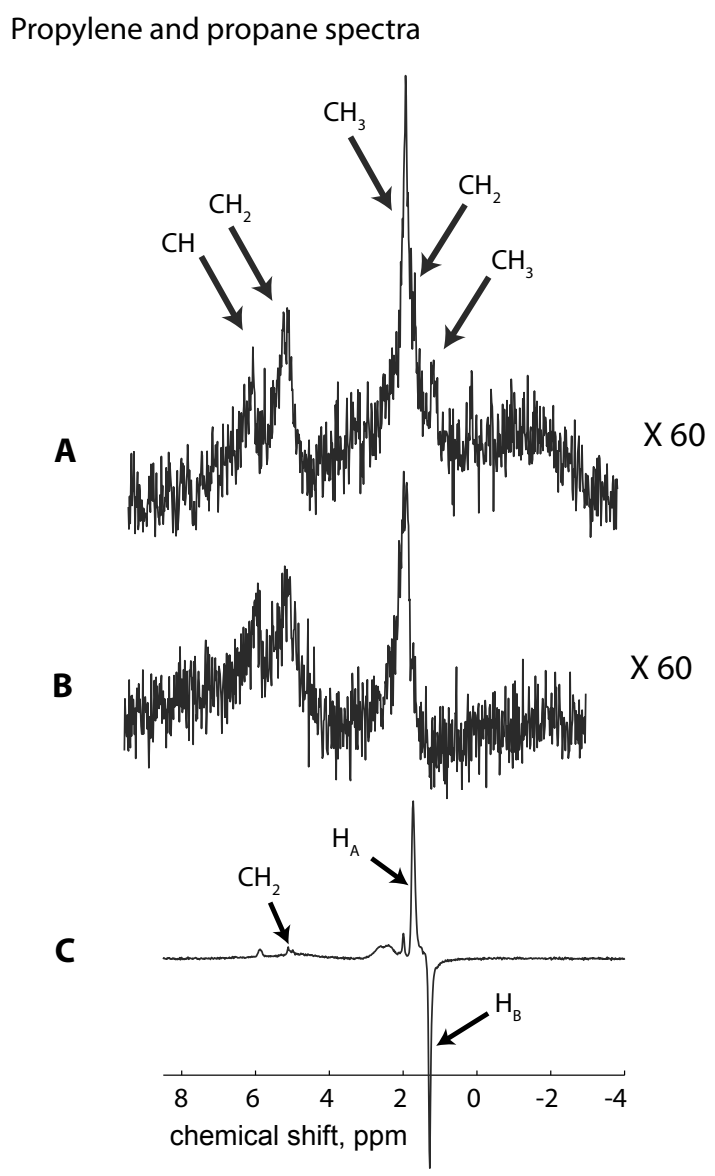

Figure 3.4: ${ }^{1} \mathrm{H}$ NMR spectra of (A) fully relaxed and thermally polarized mixture of propylene and propane acquired $2 \mathrm{~min}$ after stopping the flow, (B) propylene under flowing conditions, and (C) a mixture of propylene and the polarized propane gas under flowing conditions and with heating of the catalyst. The peaks from the $p-\mathrm{H}_{2}$-derived protons and the $\mathrm{CH}_{2}$ peak in the unreacted propylene are marked in spectrum (C). Spectra (A) and (B) have been magnified 60 times. 
Flow In A Regular Phantom

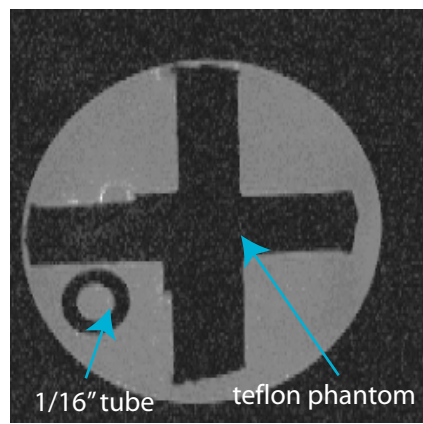

A Proton Water Image

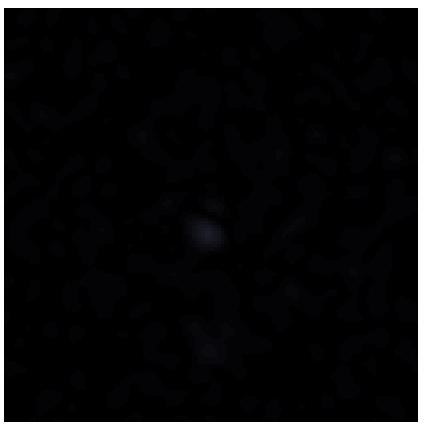

B Propene Gas-Phase Image

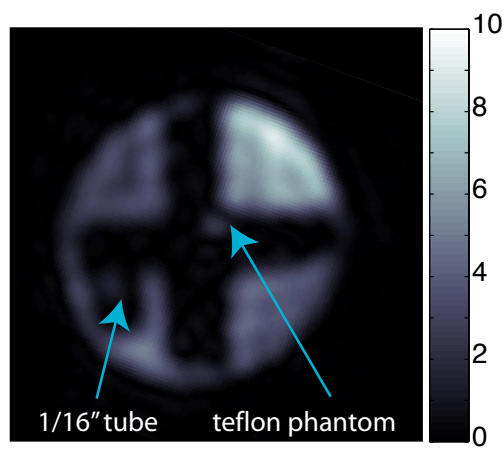

C ALTADENA Image
Flow In A Porous Medium
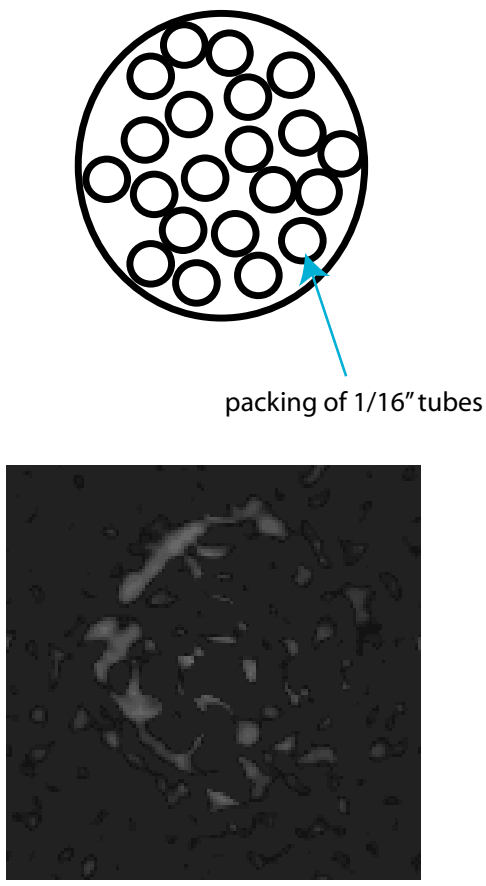

D Propene Gas-Phase Image

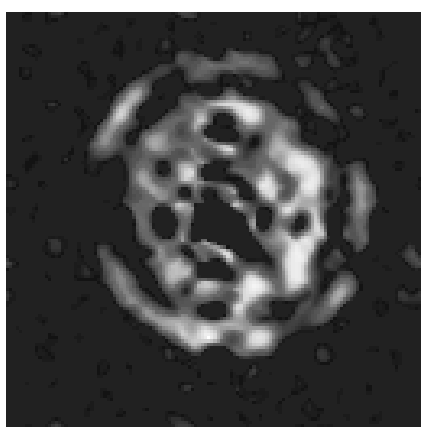

E ALTADENA Image

Figure 3.5: (A) High-resolution water proton image for a thin cross-sectional slice through a cross-shaped Teflon phantom. The circle depicts the Teflon tube $(d=1 / 16$ ") which delivers the gas. (B) Propylene gas phase image based on the signal intensity from the $\mathrm{CH}_{2}$ peak. (C) ALTADENA image from the propane $\mathrm{CH}_{3}$ peak. Parts $(\mathrm{B})$ and $(\mathrm{C})$ were reconstructed from the same experiment and are plotted with respect to the same color scale. Images (D) and (E) correspond to a phantom consisting of a random packing of capillaries to demonstrate gas-phase MRI through a porous medium. 
propylene protons is about $30: 1$. The enhancement factor is therefore $30 \times 20 \times 1 / 2=300$, an enhancement of two orders of magnitude relative to the thermally polarized gas.

It is also important to note that these experiments were not optimized. The imaging experiments, for example, could use more efficient pulse sequences, which include shorter recycle times [43] and frequency-selective pulses [65] that excite only the ALTADENA resonances followed by frequency-encoded readouts or even single-shot readouts. Further optimizations to the polarizer could be made by maximizing the surface-to-volume ratio of the supported catalyst bed or by adjusting the flow velocity, temperature, and pressure of the reaction. We note that the enhancement factor of 300 is considerably less than the theoretical ALTADENA enhancement of $\approx 16,000$ expected from $50 \%$ polarized $p-\mathrm{H}_{2}$. This is due to a combination of the short $T_{1}$ relaxation of propane protons, the dipolar relaxation in the intermediate dihydride, and relaxation during contact of the polarized product with the porous catalyst support.

A broader range of applications of this heterogeneous reaction would be possible if the gas were condensed into a liquid by cooling following the hydrogenation. Because the singlet state of $p-\mathrm{H}_{2}$-derived product can be preserved for very long periods of time in the Earth's field [45], [46] the product can be stored prior to its usage. At the time of use, the hydrogenated substrate can be transported to the high magnetic field (ALTADENA effect) or suddenly de-phased by a non-adiabatic field to create the incoherent $\mathbf{I}_{z} \mathbf{S}_{z}$ state for subsequent use in low magnetic field environments. It is also possible to transfer polarization to ${ }^{13} \mathrm{C}$ nuclei $[9,26,86]$ for lifetime enhancement and then transfer it back to ${ }^{1} \mathrm{H}$ nuclei for detection. Applications to medical imaging [108], such as lung imaging, are also possible 
if issues of gas toxicity can be addressed. A further threefold enhancement in the signal intensity is also achievable by using $100 \% p-\mathrm{H}_{2}$.

In summary, we have illustrated the application of $p-\mathrm{H}_{2}$-induced polarization with a supported catalyst to magnetic resonance imaging in the gas phase. Situations in which polarization enhancements can be envisaged include micro-fluidic flow, imaging of lungs and other porous materials, as well as MRI in low magnetic fields. 


\section{Chapter 4}

\section{Micro-reactor Characterization}

\section{using Heterogeneous PHIP}

The results in this chapter were published previously [34], and are presented here with the kind permission of the authors. Furthermore, the published results represent only a fraction of the experimental data collected over the summer of 2007. Chapters 5 and 6 present additional results from these same experiments.

\subsection{Introduction}

\subsubsection{Packed Bed Catalytic Micro-reactors}

Catalysis is a fundamental component to many industrial processes and, consequently, the optimization of catalytic reactions and reactors attracts considerable technological effort and financial commitment. An important aspect of this optimization is to 
correlate the spatial distribution of the reactive conversion inside the reactor with the morphology and packing of the catalyst. Here, we describe a spectroscopic method for this purpose based on magnetic resonance imaging [98] that uses hyperpolarized spins derived from $p-\mathrm{H}_{2}[37,118]$. Specifically, we achieve high-resolution, spatially resolved profiles of heterogeneous hydrogenation reactions taking place at a solid-gas interface inside a microreactor. We demonstrate strongly enhanced nuclear magnetic resonance signal intensities in the gas phase as well as precise control over the spatiotemporal dynamics of the polarization. The enhanced sensitivity is particularly important for tracking gases and products in small volumes (e.g., in micro-fluidic devices $[55,153]$ or the limited void space of a tightly packed catalyst bed). Moreover, the controlled delivery of $p-\mathrm{H}_{2}$-induced nuclear spin polarization acts as a spin label that can transport polarization to remote regions in the reactor. This work has implications for studying kinetics and mechanisms of multistep heterogeneously catalyzed reactions and fluid-flow transport, as well as heat and mass transfer. Such characterization should facilitate improved reactor and catalyst design.

Methods to optimize micro-reactors would be welcome in the context of microfluidic (lab-on-a chip) technology. In recent years, the compelling advantages of micro-fluidic technology $[55,153]$ in biopharmaceutical applications, chemical analysis [21], organic synthesis $[77,152]$ and industrial catalysis have been recognized and demonstrated $[5,90]$. These include smaller volumes, substantial economic savings, precise control of reactant delivery, improved fluid transport and heat dispersion, control of reaction rates, enhanced safety of highly exothermic or explosive processes, as well as the possibility of faster reactions, improved yield and chemoselectivity. Recent developments in micro-fluidic technology also 
provide powerful means for performing complex reactions, such as multistep transformations [5] or multiphase reactions [90]. Furthermore, the use of catalysts immobilized on solid supports in flow-mode micro-reactors has recently been shown to result in highly efficient reactions $[33,90]$.

\subsubsection{Methods to Characterize Micro-reactors}

One important characterization for the optimization of catalyst bed reactors is the flow map. Knowledge of the velocity distribution of the reaction product enables study of transport phenomena within a micro-reactor $[74,97,160]$ and optimization of the catalyst packing and reactor geometry. High flow uniformity in a catalytic converter is crucial for avoiding non-uniform deactivation of the catalyst caused by both chemical and thermal aging.With the increasing sophistication of mathematical modeling, such systems are routinely characterized by computational fluid dynamics. However, the relevance of such models is in doubt until they are validated against real measurements [144]. This is particularly difficult in packed-bed micro-reactors where the methods available are limited to tracers and bulk (average) properties of temperature, conversion, and velocity. Despite the need for validation, the spatio-temporal distribution of reactants and products in heterogeneous systems has been seldom visited by chemical engineering researchers in recent years, owing to the substantial difficulties with performing quantitative measurements in situ [144].

Various techniques have been used to study the motion of fluids in catalytic processes. For example, capacitance [116], single-photon emission-computed tomography [19], and positron emission tomography [123] are used to monitor gas-liquid distributions in 
multiphase reactors. But these methods measure average flow properties and have limited spatial resolution. The use of MRI to characterize micro-reactors is advantageous because the technique is noninvasive, can probe optically opaque media, and is appealing for catalysis because of the variety of molecular parameters that can be mapped with considerable chemical and spatial selectivity [43]. Specifically, flow maps and local density profiles can be generated, molecular mobility can be tracked, and chemical reaction mechanisms can be probed with spin-labeled nuclei [12]. Previous applications of MRI to heterogeneous catalysis included studies of hydrogenation processes without $p-\mathrm{H}_{2}[92,133]$, catalyst morphologies and synthesis techniques [93,107], and fluid flow through the catalyst bed [106], as well as monitoring of esterification reactions $[97,160]$ in situ. All of these applications were based on the NMR signal detection of the liquid phase and thus offer a sensitivity that is three orders of magnitude larger, as compared with gases, resulting from the difference in density. The sensitivity enhancement offered by $p-\mathrm{H}_{2}$-induced polarization (PHIP) is essential for the application of MRI to heterogeneous chemical reactions in the gas phase.

\subsubsection{Imaging Parahydrogen Induced Polarization}

As discussed in sec. $1.3, p-\mathrm{H}_{2}[36,37,118]$ is characterized by a singlet nuclear spin wave function and is relatively easy to prepare in quantum-state ensembles of very high purity. The total nuclear spin angular momentum of this state is zero, resulting in no observable NMR signal. However, if the protons participate in pair-wise hydrogenation in which they become magnetically inequivalent, strong observable magnetization can be

produced $[36,37,118]$ with a signal enhancement factor typically on the order of $10^{4}$. For 
instance, if $p-\mathrm{H}_{2}$ is used in the hydrogenation of propylene into propane [35,91], the $p-\mathrm{H}_{2-}$ derived protons will be in the singlet state immediately after the transformation of the substrate molecule into the product molecule. Typically, the molecular additions occur at randomly distributed times, and the result is an incoherent but highly polarized nuclear spin state. Application of suitable RF pulses results in a hyperpolarized NMR signal. However, if an isotropic mixing sequence is applied during the course of the reaction, not only is a coherent singlet state preserved, but the lifetime of this state is also increased [45]. As we demonstrate below, this effect allows one to create a relatively long-lived, coherent packet of polarized product molecules, which augments the versatility of this technique beyond hydrogenation reactions.

To demonstrate the effectiveness of heterogeneously catalyzed PHIP in microreactors, we use two model catalytic reactors (fig. 4.1). In both cases, propylene and $p-\mathrm{H}_{2}$ gases are flowed through the catalyst bed and react to form propane. The first reactor (reactor 1) contains a tightly packed bed of the silica gel-immobilized Wilkinson's catalyst located between two layers of glass beads. This reactor is used to produce a highly polarized product (propane) and illustrates the ability to resolve flow maps, active regions in the catalyst bed and controlled transport of polarization out of the catalyst bed. The second reactor (reactor 2) comprises powdered Wilkinson's catalyst loosely packed with some small $(\sim 2 \mathrm{~mm})$ air gaps. This second reactor further demonstrates the control of a polarized outstream and applications to a more heterogeneous packing.

The PHIP signal in these experiments is larger than the corresponding thermal signal by a factor of 300 . This enhancement is crucial in light of the coil-sample arrangement. 


\section{Reactor 1}

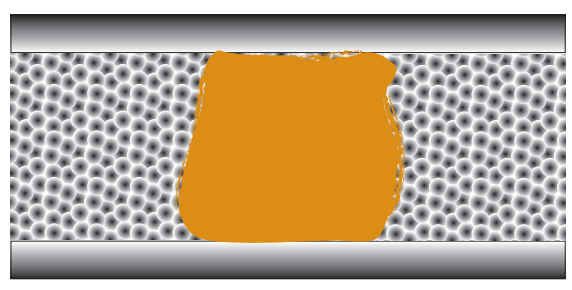

\section{Reactor 2}

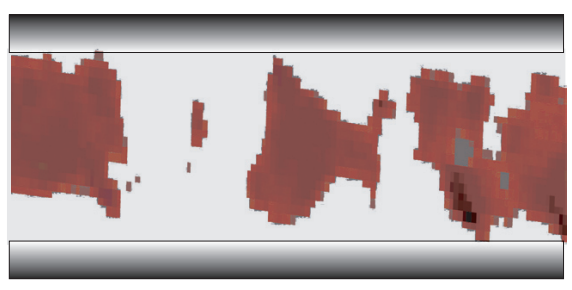

Figure 4.1: Schematic of the micro-reactors. The two model catalytic reactors consist of catalyst layers sandwiched between layers of glass beads for stability. Reactor 1 contains a thin layer of silica gel-supported Wilkinson's catalyst (orange). Reactor 2 contains powdered Wilkinson's catalyst (red) arrayed in variously sized clumps to demonstrate a more heterogeneous scenario.

The ratio of the RF coil volume occupied by the micro-channel to the RF coil volume is less than 0.01 , a situation that is unfavorable in terms of detection sensitivity. Furthermore, the presence of catalyst powder occludes the space inside the micro-channel. This sensitivity loss, combined with the low density of the gas, leads to a million-fold loss in sensitivity relative to standard liquid-state NMR. Our experimental results show that PHIP can, to a large extent, circumvent the problem of low sensitivities in gas-phase micro-reactors. 


\subsection{Results}

\subsubsection{Imaging Active Regions in the Catalyst Bed}

An image of the first reactor is shown in fig. 4.2. The spatial distribution of reactant (propylene) is barely resolved (fig. 4.2A), whereas the polarized product (propane) shows a strongly enhanced signal (fig. 4.2B). We also compare a spectrum from the reactor (fig. 4.6A) and localized spectra from upstream of (fig. 4.6B) and in (fig. 4.6C) the catalyst bed. The use of a hyperpolarized substance was also necessary for producing the high resolution gas-phase flow map shown in fig. 4.2C. This example reveals heterogeneous flow patterns in the catalyst bed, which are consistent with a non-uniform packing of the catalyst, that are not apparent in fig. 4.2B. Flow imaging at the same resolution and sensitivity would not be possible with the exceedingly weak thermal signals.

\subsubsection{Controlled Transport of Polarization within the Catalyst Bed}

As illustrated in fig. 4.3A, the residence time inside the tightly packed region leads to nearly complete magnetic relaxation, and no polarized product is observed beyond the catalyst bed. Although this outcome is ideal for imaging the active regions of the catalyst bed, the polarized spins cannot be used for subsequent reactions. Isotropic mixing sequences have been shown to prolong the lifetime of the nuclear spin-singlet state [45]. Thus, the use of an isotropic mixing sequence [135] applied for a sufficiently long duration $T_{m}$ allows the polarized product to escape the catalyst bed (the optimal $T_{m}$ will depend on the average flow velocity $\mathrm{v}$ ). This period is followed by a delay $T_{d}$, allowing the singlet state to travel a distance $x=\nu \times T_{d}$, during which the singlet state evolves into a state observable by 


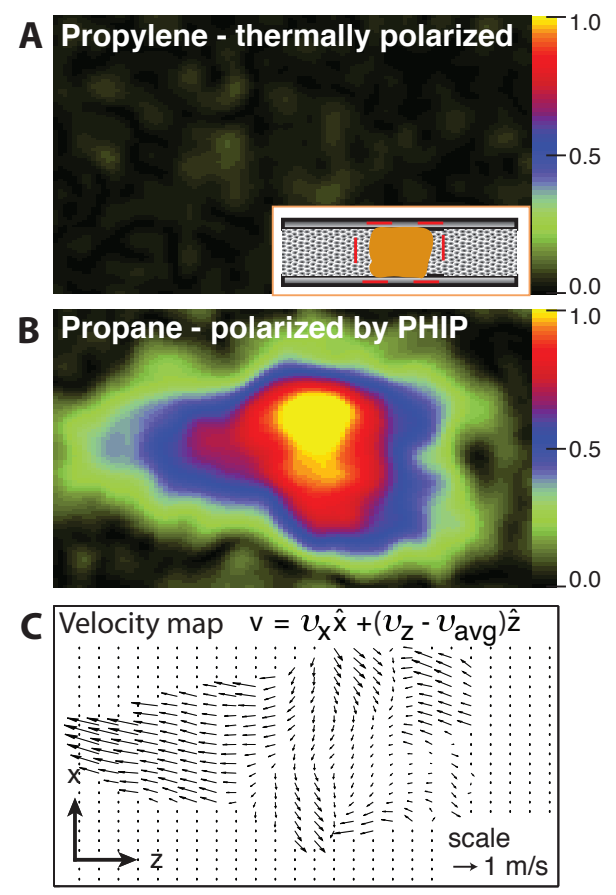

Figure 4.2: Density of active catalyst and flow map imaging. MRI images (field of view (FOV) ( $x$ to $z$ ): $2.3 \mathrm{~mm}$ by $7.0 \mathrm{~mm}$; pixel size: $24 \mu \mathrm{m}$ by $64 \mu \mathrm{m}$ ) of a tightly packed catalyst bed (catalyst layer thickness is $\sim 5 \mathrm{~mm}$ ) are shown. (A) Thermally polarized propylene. The approximate position of the imaging FOV is indicated by the dashed red lines in the inset. (B) $p-\mathrm{H}_{2}$ polarized propane. The signal-to-noise ratio (SNR) of this image is a factor of 300 larger than that of the thermally polarized propylene image. (C) Flow map in the $x z$ plane with the use of polarized propane. The orientation of the arrows represents the direction of the velocity, and the length represents the magnitude. Note that the $\hat{z}$ direction actually represents the difference from the average $z$-velocity to highlight the flow heterogeneity. Also, the resolution of the flow map is intentionally decreased by retaining only 1 of every 16 arrows to avoid excessive overlap. The SNR of thermally polarized propylene was insufficient to generate a velocity map. 

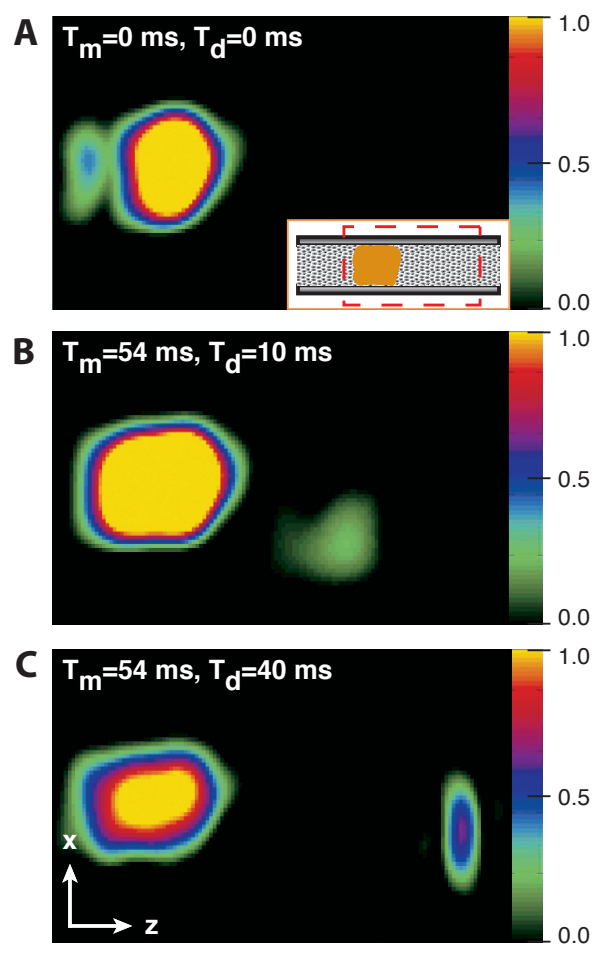

Figure 4.3: Controlled transport of polarized product. MRI images [FOV ( $x$ to $z): 8.5 \mathrm{~mm}$ by $23 \mathrm{~mm}$; pixel size: $0.5 \mathrm{~mm}$ by $1 \mathrm{~mm}$ ] for different travel times after an isotropic mixing sequence are shown. (A) No isotropic mixing: The polarized product is observed only in the catalyst layer. The approximate position of the imaging FOV is indicated by the dashed red lines in the inset. (B) Ten milliseconds after isotropic mixing, the polarized product travels $5 \mathrm{~mm}$. (C) Forty milliseconds after isotropic mixing, the product has traveled $10 \mathrm{~mm}$. 
NMR. By varying $T_{d}$, we control the distance traveled before the polarization is released. Figure 4.3, B and C, demonstrates the delivery of polarized product beyond the catalyst bed for two values of $T_{d}$.

Imaging of the second reactor (fig. 4.4A) further demonstrates the usefulness of controlling the polarized outstream. In contrast with the packing in reactor 1 , the looser packing in reactor 2 results in a polarized product that can be seen escaping the first catalyst layer and flowing to the second layer in the absence of singlet state preservation $\left(T_{m}=0 \mathrm{~ms}\right.$, $\left.T_{d}=0 \mathrm{~ms}\right)$. Although this situation is desirable when the polarized product is to be used in subsequent reactions, it is problematic as a means for imaging the catalyst layer because of poor contrast and blurring from the uncontrolled flow of polarized product. This drawback can be remedied by controlling the singlet state as follows. For short values of $T_{d}(1 \mathrm{~ms})$, no polarized product can be seen between the catalyst layers, because the singlet state has not yet evolved into an observable state. At longer time intervals $\left(T_{d}=100 \mathrm{~ms}\right)$, the polarized product has traversed longer distances and evolved into an observable polarized product. A photograph of the reactor (fig. 4.4B) provides a comparison of the distribution of catalyst in the reactor with the structural morphology observed in the MRI images.

Regardless of the packing, singlet state preservation provides a distinctive method for controlled delivery of polarization. Combined with knowledge of the local flow velocity, the timed release of polarization can be performed in remote parts of a micro-reactor. Thus, in multistep reactions, the polarized product can be used as a spin label to elucidate subsequent stages of a reaction [12]. 

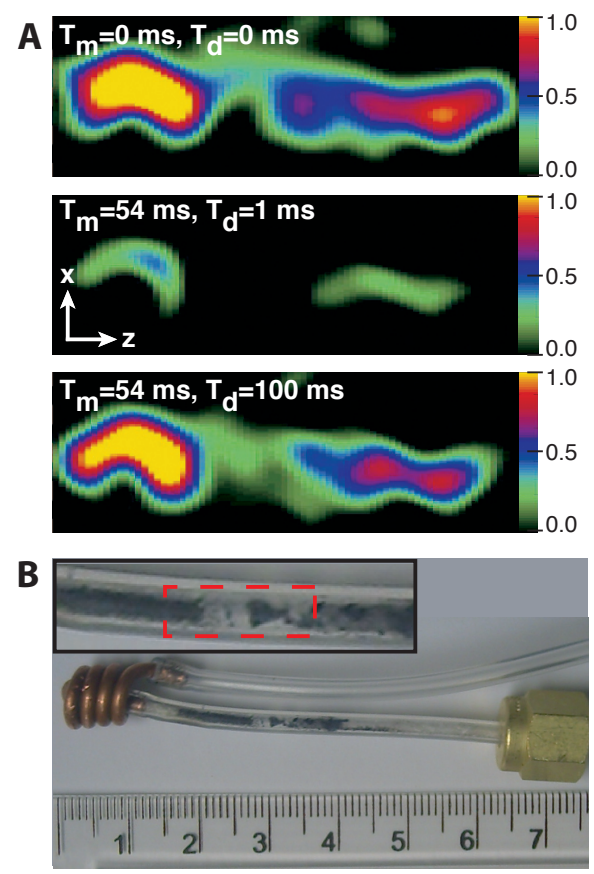

Figure 4.4: Imaging heterogeneous distributions of catalyst. (A) MRI images [FOV ( $x$ to $z): 2 \mathrm{~mm}$ by $15 \mathrm{~mm}$; pixel size: $0.4 \mathrm{~mm}$ by $0.9 \mathrm{~mm}$ ] of the heterogeneous packing, acquired without isotropic mixing $\left(T_{m}=0 \mathrm{~ms}, T_{d}=0 \mathrm{~ms}\right)$ and for two different travel time intervals $\left(T_{d}=1\right.$ and $\left.100 \mathrm{~ms}\right)$ after $54 \mathrm{~ms}$ of isotropic mixing. (B) Catalytic reactor containing a heterogeneous packing of Wilkinson's catalyst powder mixed with glass beads. An enlarged view of the catalyst packing is shown in the inset. The dashed red lines indicate the approximate location of the FOV for the images in (A).

\subsection{Future Applications}

These experiments can be extended by means of a variety of polarization transfer and spin manipulation methods to move the enhanced signal to other NMR-active nuclei on the substrate molecule or onto the catalyst itself $[12,74]$. Transfer of polarization to heteronuclei yields a larger range of chemical shifts as compared with that of protons [74]. The highly polarized spin product also makes it possible to image flows [6] and reactions [50] in precisely engineered micro-channels non-invasively, opening the way for a variety of microfluidic applications. 
There are still many challenges to address before these results can be extended to a wider range of hydrogenation reactions and conditions of industrial catalytic processes. In particular, it remains to be seen to what extent the polarization lifetimes and the NMR line widths of the reaction products are affected by the presence of metal catalyst surfaces, micro-scopic gradients of magnetic susceptibility, high temperatures, paramagnetic catalysts and impurities, liquid films, and other complications that can be expected under conditions encountered in practice. To this end, recent results [94] that demonstrate PHIP in heterogeneous hydrogenations catalyzed by supported metal catalysts $\left(\mathrm{Pt} / \mathrm{Al}_{2} \mathrm{O}_{3}\right.$ and $\left.\mathrm{Pd} / \mathrm{Al}_{2} \mathrm{O}_{3}\right)$ are encouraging.

\subsection{Appendix}

\subsubsection{Equipment}

MRI was carried out on a vertical-bore $300 \mathrm{MHz}$ Varian Inova NMR spectrometer (Varian Inc., Palo Alto, CA) equipped with micro-imaging gradients (max. strength: $80 \mathrm{G} / \mathrm{cm}$ along $x, y$ and $60 \mathrm{G} / \mathrm{cm}$ along $z)$ and a commercial Varian $10 \mathrm{~mm}$ RF probe $(\pi / 2$ pulse width was $50 \mu \mathrm{s})$.

\subsubsection{Parahydrogen Production}

A $\mathrm{H}_{2}$ mixture enriched in the para spin state $\left(50 \% p-\mathrm{H}_{2}\right)$ was produced by passing pure hydrogen gas through a $\mathrm{Cu}$ cell, packed with $\mathrm{FeO}(\mathrm{OH})$ and immersed in a liquid $\mathrm{N}_{2}$ filled dewar. The ortho:para ratio was verified by measuring the NMR signal intensities of $\mathrm{H}_{2}$ in a cell packed with porous alumina $\left(\mathrm{Al}_{2} \mathrm{O}_{3}\right)$, the method being detailed in sec. A.1.3. 
An aluminum cylinder was filled with the para-enriched- $\mathrm{H}_{2}$ to $80 \mathrm{psig}$ and the propylene was then added to a total pressure of 100psig. Consequently, the volumetric composition in the reactant mixture was $20 \%$ propylene, $40 \% p-\mathrm{H}_{2}$ and $40 \% o-\mathrm{H}_{2}$.

\subsubsection{Supported Catalyst and Reactor}

The immobilized catalyst [Wilkinson's catalyst supported on modified silica gel, $\left.\mathrm{RhCl}\left(\mathrm{PPh}_{3}\right)_{2} \mathrm{PPH}_{2}\left(\mathrm{CH}_{2}\right)_{2}-\mathrm{SiO}_{2}\right]$ was prepared by adding an approximately stoichiometric amount of 2-diphenylphosphinoethyl-functionalized silica gel to a toluene solution of Wilkinson's catalyst, according to the procedure described in previous work [91]. About 0.1g of the catalyst was packed inside a section of $1 / 8$ " nylon tubing, surrounded by two layers of $200 \mu \mathrm{m}$ diameter glass beads. The packing was done using a small metal wire and is expected to be non-uniform. The reactor was held at approximately $145^{\circ} \mathrm{C}$ by using the variable temperature controller to preheat the gas flowing through a heat exchange coil. The hot gas then flowed directly into the catalyst bed. The heat exchanger for the microreactor was made by coiling 0.08 " OD Cu tubing and melting $1 / 8$ " OD nylon tubing to the ends. Activation of the catalyst requires 12 hours at $145^{\circ} \mathrm{C}$ under $15 p s i g$ of the gas mixture, resulting in conversion of the propylene.

\subsubsection{Imaging Pulse Sequence}

The imaging pulse sequence is shown in fig. 4.5. Typical excitation pulses $(\pi / 4)$ were $25 \mu$ s in duration. Typical scan times with a spatial encoding matrix size of $25 \times 19$ were on the order of a minute. This scan time can be reduced by selectively exciting a resonance of interest followed by rapid imaging sequences. We used the pure phase 
Spectroscopic imaging with velocity encoding

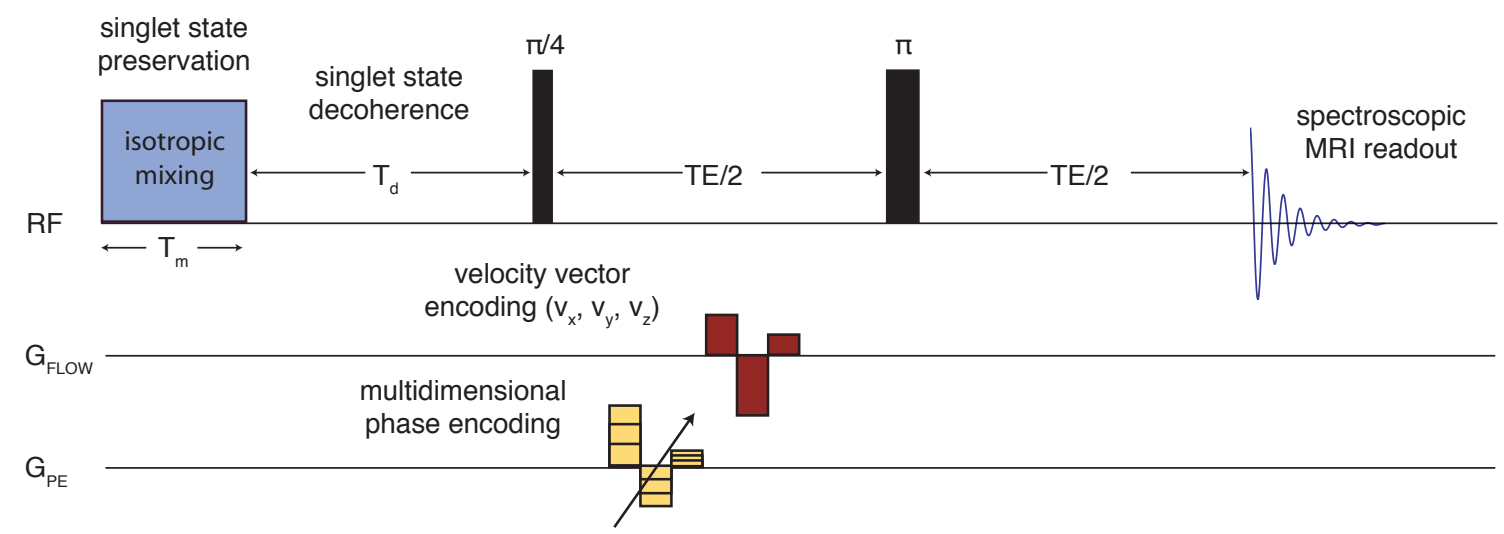

Figure 4.5: Flow-encoded MRI pulse sequence for heterogeneous reaction imaging and controlled delivery of spin polarization.

encoding protocol to enable 2D chemical-shift selective imaging with good spatial resolution. Position and velocity encoding were performed using pulsed field gradients (pfg) consisting of three pulses with equal widths and a total encoding time of $800 \mu \mathrm{s}$. In general, the spatial resolution is improved by the use of short phase-encoding gradient pulses combined with careful adjustments of pulse amplitudes to eliminate artifacts arising from the velocity and acceleration of the gas molecules (see below). The velocity encoding pfg was similar to the position encoding pfg except the relative intensities of the three pulses were chosen to be sensitive to velocity only and not position or acceleration.

Isotropic mixing was performed using the decoupling in the presence of $s$ calar interactions (DIPSI-3) sequence [135]. The choice of DIPSI-3 was arbitrary, and is one of many examples of sequences preserving the singlet state [45] by creating a relaxation-free environment. This phenomenon was first explained by Bowers and Weitekamp $[36,37]$ and subsequently exploited by others [45]. 
The spin echo readout serves several purposes. First, it refocuses the evolution due to magnetic susceptibility gradients, thereby mitigating the attenuation of signal at the first point of the FID. Second, a nonzero echo time $(T E)$ removes the broad proton resonances from the $o-\mathrm{H}_{2}$ and solid components. Third, it allows a certain amount of evolution under the $\mathrm{J}$ coupling, with the end result of converting some of the anti-phase magnetization into in-phase magnetization. The in-phase character makes it easier to integrate the peaks which are anti-phase when PHIP is performed at high magnetic fields [36,37]. Ultimately, $T ! E$ cannot be too long since $T_{2}$ relaxation will eventually dominate. These qualitative arguments led us to pick the following $T ! E$ values: $16.7 \mathrm{~ms}$ for the data of fig. $4.2,5 \mathrm{~ms}$ for fig. 4.3 and $2.5 \mathrm{~ms}$ for fig. 4.4 . This choice was made out of convenience and is not necessarily the most optimal.

\subsubsection{Parahydrogen Induced Polarization and Singlet Lifetime}

Parahydrogen is characterized by a singlet nuclear spin wavefunction,

$$
|\Psi\rangle=\frac{1}{\sqrt{2}}(|\alpha \beta\rangle-|\beta \alpha\rangle)
$$

, where $|\alpha\rangle$ and $|\beta\rangle$ represent the eigenstates of the spin angular momentum operator parallel to the direction of the magnetic field. It is described by a density operator, $\rho=|\Psi\rangle\langle\Psi|=$ $\mathbf{1} / 4-\mathbf{I} \cdot \mathbf{S}$, where $\mathbf{I}$ and $\mathbf{S}$ are spin angular momentum vector operators, $\mathbf{I} \cdot \mathbf{S}=\mathbf{I}_{x} \mathbf{S}_{x}+$ $\mathbf{I}_{y} \mathbf{S}_{y}+\mathbf{I}_{z} \mathbf{S}_{z}$ and $\mathbf{1} / 4$ represents the maximally mixed state $(1 / 4$ times the unit operator 1). In contrast, the thermal equilibrium state is $\rho=1 / 4+\varepsilon\left(\mathbf{I}_{z}+\mathbf{S}_{z}\right)$, where $\varepsilon$ is a very

small number $\left(10^{-4}\right.$ to $\left.10^{-5}\right)$ for typical fields and temperatures. Although the spins of the singlet state are highly ordered, no NMR signal is observable because the total nuclear 
spin angular momentum is zero. An equivalent explanation is to say that $\rho$ commutes with the Hamiltonian. If the protons participate in pair-wise hydrogenation in which they become magnetically inequivalent, observable magnetization can be produced with a signal enhancement factor on the order of $1 / \varepsilon$ (typically $\left.10^{4}\right)$.

Immediately after the reaction, the $p-\mathrm{H}_{2}$ derived protons are in the singlet state, $\rho=\mathbf{1} / 4-\mathbf{I} \cdot \mathbf{S}$. If the reaction proceeds at random times, the distribution of these random initial states leads to an incoherent averaging $\left(\mathbf{I}_{x} \mathbf{S}_{x}+\mathbf{I}_{y} \mathbf{S}_{y}\right)=0$ of this part of the density operator, leaving us with $\rho=\mathbf{1} / 4-\mathbf{I}_{z} \mathbf{S}_{z}$. The fact that the initial state of the parahydrogeninduced polarization (PHIP) is a singlet has important consequences. If an isotropic mixing sequence, such as DIPSI (discussed above), is applied during the course of the reaction, the effective Hamiltonian is of the form of a scalar coupling, $H=J \mathbf{I} \cdot \mathbf{S}$. Most importantly, the part $I_{z}-S_{z}$ of the Hamiltonian, due to the chemical inequivalence of the spins, which would otherwise lead to a dephasing of the $\mathbf{I} \cdot \mathbf{S}$ state is strongly suppressed. In other words, the singlet state no longer averages to $\rho=\mathbf{1} / 4-\mathbf{I}_{z} \mathbf{S}_{z}$. The end result of this procedure is analogous to removing some relaxation pathways resulting in a longer lifetime of the singlet state [45]. This allows one to create a relatively long lived, coherent packet of polarized product molecules.

\subsubsection{Pulsed Field Gradients}

As noted above, both the position and velocity encoding pulsed field gradients (pfg) were composed of three rectangular pulses. The relative amplitudes of these pulses can be chosen to compensate for artifacts arising from the gas flowing during the encoding 
time. Under conditions where a group of spins located at position $\mathbf{r}(\mathrm{t})$ are moving in a time-dependent gradient, $\mathbf{G}(\mathrm{t})$, the phase of the NMR signal at time $T$ is given by

$$
\phi_{\text {tot }}=\gamma \int_{0}^{T} \vec{G}(t) \cdot \vec{r}(t) \mathrm{d} t
$$

where the position vector can be expressed using the second-order Taylor formula,

$$
\vec{r}(t)=\vec{r}_{0}+\vec{v}_{0} t+\frac{1}{2} \vec{a}_{0} t^{2}+O\left(t^{3}\right)
$$

Inserting this expression into eq. 4.2 gives the decomposition

$$
\phi_{\text {tot }}=\gamma \vec{r}_{0} \cdot \int_{0}^{T} \vec{G}(t) \mathrm{d} t+\gamma \vec{v}_{0} \cdot \int_{0}^{T} \vec{G}(t) t \mathrm{~d} t+O\left(T^{3}\right)=\phi_{\text {pos }}+\phi_{\text {vel }}+O\left(T^{3}\right)
$$

where $\phi_{\text {pos }}$ is the phase accrued over the time interval $[0, T]$ due to the initial position and $\phi_{v e l}$ is that due to initial velocity.

In terms of the $n$-th moment, $\vec{M}_{n}=\int \vec{G}(t) t^{n} \mathrm{~d} t$, the phase-encoding gradient is made insensitive to velocity and acceleration by setting $\vec{M}_{1}=\vec{M}_{2}=0$. On the other hand, velocity encoding is achieved by a gradient which has the property $\vec{M}_{0}=\vec{M}_{2}=0$. Higher order moments, $n>2$, are safely ignored under the conditions of steady flow used in this experiment. Our pfg employed $10 \mu$ s rise times and $246.3 \mu$ s pulses for a total encoding duration of $800 \mu \mathrm{s}$. If the amplitudes (in $\mathrm{G} / \mathrm{cm}$ ) of the three pulses are labeled $g_{1}, g_{2}$, $g_{3}$ for the initial, second and third pulses, respectively, then the method outlined above gives $g_{2}=-(7 / 11) g_{1}$ and $g_{3}=(2 / 11) g_{1}$ for compensated position encoding. Similarly, the compensated velocity encoding pfg requires $g_{2}=-(3 / 2) g_{1}$ and $g_{3}=(1 / 2) g_{1}$. The resulting proportionality between phase and position or velocity is $\phi_{v e l}=0.57 \mathrm{G}^{-1} \vec{g}_{\text {pos }} \cdot \vec{r}_{0}$ and $\phi_{v e l}=1.4 \times 10^{-4} \mathrm{~s} / \mathrm{G} \vec{g}_{v e l} \cdot \vec{v}_{0}$. Acquiring several images with different values for $\vec{g}_{p o s}$ and 
$\vec{g}_{v e l}$ allows for Fourier reconstruction of the position and velocity of the spins at time $t=0$.

Figure $4.2 \mathrm{C}$ was obtained using $g_{v e l, z}=0$ and $3.55 \mathrm{G} / \mathrm{cm}$ and $g_{v e l, x}=0$ and $4.83 \mathrm{G} / \mathrm{cm}$.

\subsubsection{Enhancement Factor}

The large difference between PHIP and thermal polarization is seen by comparing the image signal to noise ratio (SNR) of fig. $4.2 \mathrm{~B}$ (polarized propane) to that of fig. $4.2 \mathrm{~A}$ (thermal propylene). The image SNR for the PHIP image is greater than that of the thermal image by a factor of at least 300. A similar difference in SNR is observed in the ${ }^{1} \mathrm{H}$ spectrum (fig. 4.6A), where the PHIP peak SNR is more than 300 times the SNR of any thermal peak. The spectrum in fig. $4.6 \mathrm{~A}$ is from the entire micro-reactor.

We may also compare the SNR of propylene in the region just upstream from the catalyst bed (in the glass beads) with that of the polarized propane in the catalyst bed. This is possible with our chemical shift imaging experiment, which allows integration of a spectrum from any region in the image. Figure 4.6 compares the complete spectrum (fig. 4.6A, no imaging gradients) and the reconstructed spectra from the catalyst bed (fig. 4.6C) and the region just before the catalyst bed (fig. 4.6B). This approach gives an enhancement factor of approximately 200. The difference here, compared with the enhancement discussed above, is due to the chemical reaction occurring in the catalyst bed. This reduces the amount of propylene in the catalyst bed, further reducing the already weak thermal signal, resulting in a slightly larger apparent enhancement.

The propylene resonances are found at $1.7,4.9$, and $5.7 \mathrm{ppm}$ while the propane res-

onances are at 0.9 and $1.3 \mathrm{ppm}$ (fig. $4.6 \mathrm{~A}$ ) with a $\mathbf{J}$-splitting of $7.4 \mathrm{~Hz}$. In these experiments, 


\section{A Full spectrum, no imaging gradients}

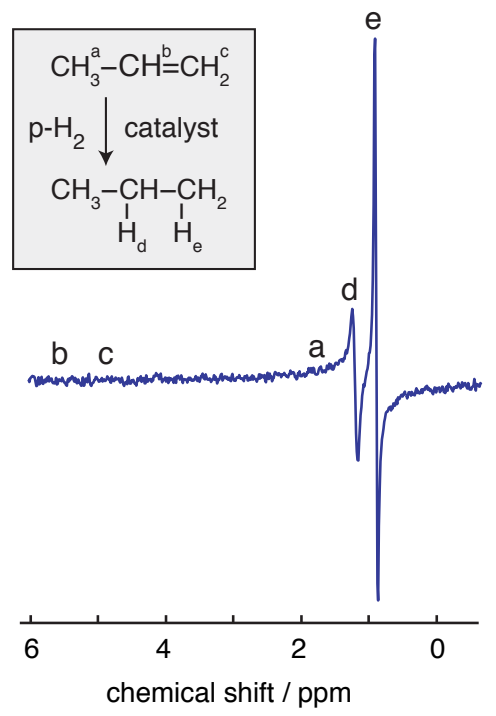

B Reconstructed spectrum, region upstream of catalyst

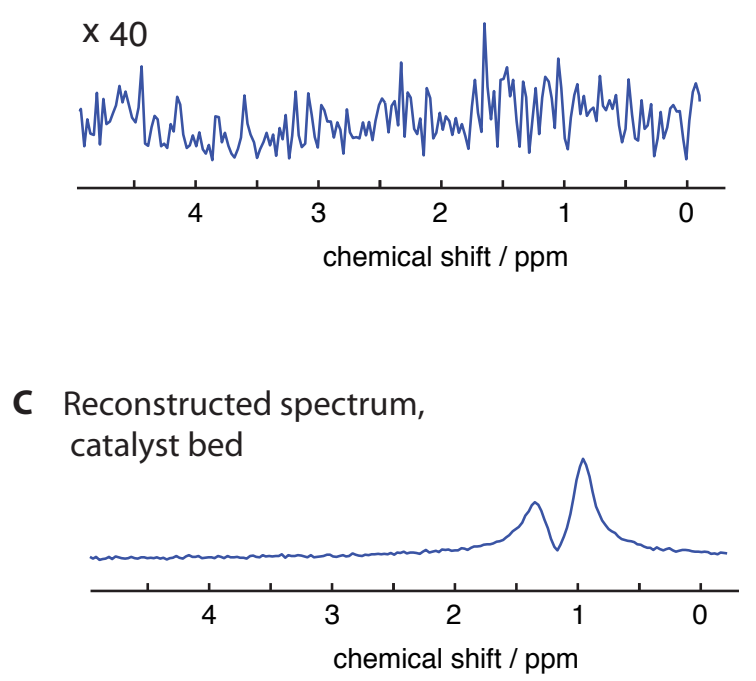

Figure 4.6: ${ }^{1} \mathrm{H}$ spectra in the micro-reactor. $\mathbf{A}^{1} \mathrm{H}$ spectrum of the micro-reactor with imaging gradients turned off. $\mathbf{B}$ and $\mathbf{C}{ }^{1} \mathrm{H}$ spectra reconstructed from a chemical shift imaging experiment. Spectrum $\mathbf{B}$ is the average signal reconstructed from the region upstream of the catalyst bed and has been scaled by a factor of 40. Spectrum $\mathbf{C}$ is the average signal reconstructed from the catalyst bed.

the proton line width without imaging gradients (fig. 4.6A) was $15 \mathrm{~Hz}$. The transverse decay time of the polarized signal is on the order of tens of milliseconds and is mainly due to temporary adsorption on the surface of the catalyst bed. This broadening combined with the anti-phase nature of the polarized signal results in a drop of sensitivity due to the partial cancellation of the antiphase signals. 


\section{Chapter 5}

\section{Mapping Temperature}

\section{Distribution in a Catalyst Bed}

\subsection{Introduction}

The micro-reactor experiments described in the previous chapter generated a large amount of data, only a fraction of which was published [34]. One of the unpublished observations that resulted in considerable interest over the past six months is a lineshape perturbation that provides temperature contrast to the catalyst bed image. The current status of these investigations is presented below and a manuscript based on these results is being prepared for publication, pending several additional experiments. 


\subsubsection{Characterizing Packed Bed Catalytic Micro-reactors}

With the rapid growth of microfluidic lab-on-a-chip technology [55,153], there is increasing interest in micro-scale reactors $[5,90]$. Section 4.1 discusses the challenges of characterizing such micro-reactors and presents an MRI-based technique for mapping the active regions of the catalyst bed, as well as obtaining the velocity map. An additional parameter that is central to reactor optimization is the temperature distribution. The temperature distribution is important for characterizing heat transport within the catalyst bed, as well as the heat of the reaction.

The advantages of using MRI to measure the temperature distribution in a packed bed micro-reactor are largely the same as those outlined in sec. 4.1.2. In summary, the catalyst powder is opaque, which limits optical methods; thermocouple-based methods provide only very low resolution maps of temperature, but most importantly, the size of the thermocouple relative to the channel disturbs the fluid flow in the catalyst bed, changing the resulting heat transfer and steady state temperature distribution. In this context, the primary disadvantages of MRI based techniques are inherent low sensitivity and the RF shielding provided by metal containers. While lab scale reactors are typically made of stainless steel, micro-reactors are typically etched in glass or silicon and are, thus, amenable to characterization by MRI microscopy.

The following results are based on the technique reported in [34] and described in chapter 4 . The sensitivity enhancement due to the $p-\mathrm{H}_{2}$ polarization permits analysis of the lineshapes, and it is the perturbation of the lineshape due to temperature that allows us to perform MRI thermometry. Without the enhanced SNR, this analysis would not be 
possible.

\subsubsection{Temperature-Dependent NMR Parameters}

To visualize temperature variation with MRI, one of the variables in the signal equation (eq. 3.1) must have a significant temperature dependence. For example, $T_{1}$ or $T_{2}$ contrast is obtained by varying the flip angle, the echo time $(T E)$ and the repetition time $(T R)$. In the case of imaging the catalyst bed, a constant flow of fresh hyperpolarized spins is used for each acquisition. Thus, the $T R$ is set by the flow rate and the initial polarization is independent of $T_{1}$. On the other hand, $T_{2}$ decay for gases is dominated by diffusion through field gradients during the encoding time. While this is expected to have some dependence on temperature, SPI reduces the manifestation of $T_{2}$ in the image to an attenuation factor (eq. 3.6), which is expected to be small for short encoding times.

Spectroscopic imaging (sec. 3.1.1) provides chemical shift and lineshape as additional parameters that can introduce contrast to the image. Both chemical shift and $T_{2}^{*}$ are expected to be temperature dependent. The experimentally observed chemical shift is an ensemble average of the chemical shift associated with each accessible vibrational and rotation state. The relative populations of these states is temperature dependent, but the resulting temperature dependence for ${ }^{1} \mathrm{H}$ chemical shifts is quite small, typically less than several thousandths of a ppm per ${ }^{\circ} \mathrm{C}[117,129] . T_{2}^{*}$, on the other hand, is dominated by field inhomogeneities due to the susceptibility-induced gradients. The heterogeneous nature of the catalyst prevents shimming away such gradients, as they vary on the $\mu \mathrm{m}$ length scale and the result is significant line broadening. However, fast motion of the nuclei over the 
length scale of these magnetic field gradients can average these inhomogeneities away. The extent of the averaging depends on the mean free path of the molecule, which scales linearly with the temperature [112]. Thus, the motional averaging of the local magnetic field gradients provides a correlation between temperature and line width. If this correlation is strong enough, it provides a means to add temperature contrast to our catalyst bed image.

\subsection{Methods}

The data used here is from the same set of experiments described in ch. 4. The experimental setup and CSI pulse sequence is given in sec. 4.4. In summary, a mixture of propylene and $p-\mathrm{H}_{2}$ gas is flowed through a small catalyst bed containing a solid catalyst powder attached to silica gel. The resulting PASADENA polarized propane is imaged via SPI (sec. 4.4.4), which provides a spectral dimension in addition to the spatial dimensions.

In order to extract the temperature map from this data, the correlation between line width and temperature must be established. This is performed using thermallypolarized propane in an NMR tube filled with 60-200 mesh silica gel. Due to the low signal strength of the gas, signal averaging is required, but the situation is not as bad as the case for micro-reactor imaging. First, the sample fills the entire RF coil, whereas the imaging experiment required an RF coil large enough to surround the entire reactor. This provides almost two orders of magnitude better sensitivity than the imaging setup. Furthermore, the sensitivity in imaging scales with the voxel resolution, $(\Delta X)(\Delta Y)(\Delta Z)=V\left(N_{x} N_{y} N_{z}\right)^{-1}$. In terms of SNR, spectroscopy has $N_{x}=N_{y}=N_{z}=1$, which provides almost three orders of magnitude sensitivity in our case. Because of this, only $N S=8$ is needed. 
We used a 5mm NMR tube with a Young Adapter containing 15psig of propane. Measurements were made on a Bruker Avance DRX spectrometer with a $34 \mu \mathrm{s} 90^{\circ}$ time $($ at $0 \mathrm{db}), T E=3 \mathrm{~ms}$ and $T R \approx 1 \mathrm{~s}$. Spectra were recorded at $40,60,80,100$ and $120^{\circ} \mathrm{C}$, with a 15min stabilization time between each measurement. The probe was re-matched and re-tuned at each temperature. The results are given below (fig. 5.3) and show a linear dependence of line width on temperature, decreasing at higher temperatures, as expected.

In order to use this line width-temperature correlation, the line widths must be accurately extracted from the experimental data. This requires a model of the spectrum. The reactor will contain both PASADENA polarized propane and thermally-polarized propane. The thermal propane results from polarized product that has relaxed, as well as from hydrogenation initiated by catalyst that was reduced to pure Rh metal during the activation process (sec. 4.4.3). Given the chemical shifts $\left(\omega_{A}=0.899 \mathrm{ppm}\right.$ and $\left.\omega_{B}=1.336 \mathrm{ppm}\right)$, the J-coupling $(7.4 \mathrm{~Hz})$, and the phase accumulated during the spin echo $(\phi=\pi J T E)$, we have

$$
\begin{aligned}
S_{\text {thermal }}(\omega)= & \frac{6}{2^{2}}\left(\mathrm{e}^{-\imath 2 \phi} \mathcal{L}\left(\omega_{A} ;-\pi J ; \lambda\right)+2 \mathcal{L}\left(\omega_{A} ; 0 ; \lambda\right)+\mathrm{e}^{+\imath 2 \phi} \mathcal{L}\left(\omega_{A} ;+\pi J ; \lambda\right)\right) \\
& +\frac{2}{2^{6}}\left(\mathrm{e}^{-\imath 6 \phi} \mathcal{L}\left(\omega_{B} ;-3 \pi J ; \lambda\right)+6 \mathrm{e}^{-\imath 4 \phi} \mathcal{L}\left(\omega_{B} ;-2 \pi J ; \lambda\right)\right. \\
& +15 \mathrm{e}^{-\imath 2 \phi} \mathcal{L}\left(\omega_{B} ;-\pi J ; \lambda\right)+20 \mathcal{L}\left(\omega_{B} ; 0 ; \lambda\right)+15 \mathrm{e}^{+\imath 2 \phi} \mathcal{L}\left(\omega_{B} ;+\pi J ; \lambda\right) \\
& \left.+6 \mathrm{e}^{+\imath 4 \phi} \mathcal{L}\left(\omega_{B} ;+2 \pi J ; \lambda\right)+\mathrm{e}^{+\imath 6 \phi} \mathcal{L}\left(\omega_{B} ;+3 \pi J ; \lambda\right)\right)
\end{aligned}
$$


for the thermal spectrum and

$$
\begin{aligned}
S_{\text {pasadena }}(\omega)= & \frac{6}{2^{2}}\left[\mathcal{L}\left(\omega_{A} ;-\pi J ; \lambda\right)+\left(\mathrm{e}^{+\imath 2 \phi}-\mathrm{e}^{-\imath 2 \phi}\right) \mathcal{L}\left(\omega_{A} ; 0 ; \lambda\right)-\mathcal{L}\left(\omega_{A} ;+\pi J ; \lambda\right)\right] \\
& +\frac{2}{2^{6}}\left[\mathrm{e}^{-\imath 4 \phi} \mathcal{L}\left(\omega_{B} ;-3 \pi J ; \lambda\right)+\left(5 \mathrm{e}^{-\imath 2 \phi}-\mathrm{e}^{-\imath 6 \phi}\right) \mathcal{L}\left(\omega_{B} ;-2 \pi J ; \lambda\right)\right. \\
& +\left(10-5 \mathrm{e}^{-\imath 4 \phi}\right) \mathcal{L}\left(\omega_{B} ;-\pi J ; \lambda\right)+10\left(\mathrm{e}^{+\imath 2 \phi}-\mathrm{e}^{-\imath 2 \phi}\right) \mathcal{L}\left(\omega_{B} ; 0 ; \lambda\right) \\
& -\left(10-5 \mathrm{e}^{+\imath 4 \phi}\right) \mathcal{L}\left(\omega_{B} ;+\pi J ; \lambda\right)-\left(5 \mathrm{e}^{+\imath 2 \phi}-\mathrm{e}^{+\imath 6 \phi}\right) \mathcal{L}\left(\omega_{B} ;+2 \pi J ; \lambda\right) \\
& \left.-\mathrm{e}^{+\imath 4 \phi} \mathcal{L}\left(\omega_{B} ;+3 \pi J ; \lambda\right)\right]
\end{aligned}
$$

for the PASADENA spectrum, where $\mathcal{L}$ is a Lorentzian lineshape, given by

$$
\mathcal{L}\left(x ; x_{0}, \lambda\right)=\frac{\lambda}{\lambda^{2}+x_{0}^{2}}-\imath \frac{x_{0}}{\lambda^{2}+x_{0}^{2}}
$$

The need for both thermal and PASADENA signals is depicted in fig. 5.1. Specifically, the spectrum obtained from the catalyst bed is clearly not pure PASADENA signal, as seen by comparing fig. 5.1A and D. It should be noted that the original idea when performing the experiments was to rephase the antiphase signal with a spin echo because antiphase peaks with $\lambda \gtrsim J$ results in a large degree of cancellation and reduction in overall signal intensity. However, the antiphase signal is only completely refocused in a two spin system. The J-coupling to the other, non PASADENA polarized spins results in some degree of antiphase line shape regardless of the TE. However, the spectrum in fig. 5.1A cannot be pure thermal signal either; the broad lines $(\approx 30 \mathrm{~Hz})$ lead to overlap of the tails between the two peaks, as shown in fig. $5.1 \mathrm{C}$, whereas the spectrum in $5.1 \mathrm{~A}$ has destructive interference between the two peaks. A mixture of thermal and PASADENA signals provides a good fit to the observed spectrum, as shown in fig. 5.1B.

The fitting was done using Matlab's nlinfit algorithm, which uses a non-linear least 
A Real spectrum
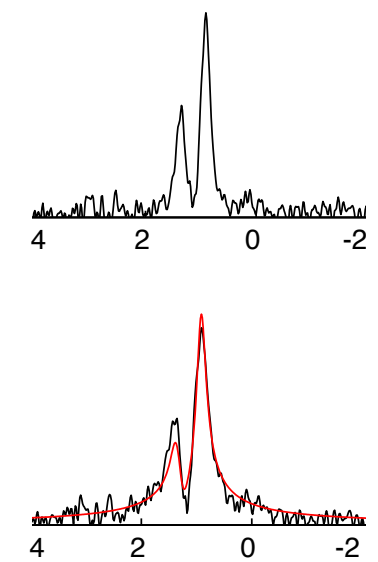

B Abs spectrum with best fit
C Thermal spectrum, simulated
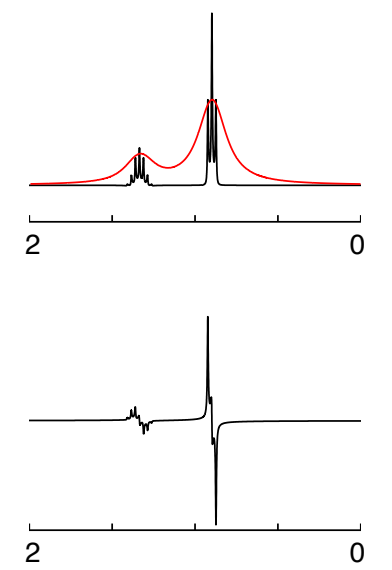

D PASADENA spectrum, simulated

Figure 5.1: Experimental and simulated propane spectra, $T E=5 \mathrm{~ms}$. (A) Real component of the micro-reactor spectrum. (B) Absolute value spectrum of the micro-reactor. (C) Real component of simulated thermal spectrum. The black spectrum has a line width of $1 \mathrm{~Hz}$ and the red spectrum has a line width of $30 \mathrm{~Hz}$ to compare with (A). (D) Real component of simulated PASADENA spectrum. The experimental spectrum is not pure PASADENA because the spin echo does not refocus the antiphase signal, as shown in (D). However, (A) is not described completely by thermal propane either; the destructive interference between the two peaks requires a mixture of both thermal and PASADENA spectra. The best fit of the spectrum requires an approximate 2:1 ratio of PASADENA signal strength to thermal signal strength. Absolute value spectra are used in the final analysis because of phase variation across the catalyst bed.

squares regression and requires an initial guess. In situations where the $\mathrm{SNR}$ is $<2$ a good initial guess is essential for obtaining a sensible answer. This initial guess is chosen by hand using a high SNR spectrum in the middle of the catalyst bed. This guess is then used for each point within the threshold mask (see below) to obtain the frequency offset $(\Delta \omega)$, line width $(\lambda)$, and relative fractions of PASADENA signal $(f)$. In other words, eq. 5.1 and 5.2 are used to construct a model spectrum

$$
S(\omega+\Delta \omega, \lambda, f)=f S_{\text {pasadena }}(\omega+\Delta \omega, \lambda)+(1-f) S_{\text {thermal }}(\omega+\Delta \omega, \lambda)
$$


A Signal to noise ratio

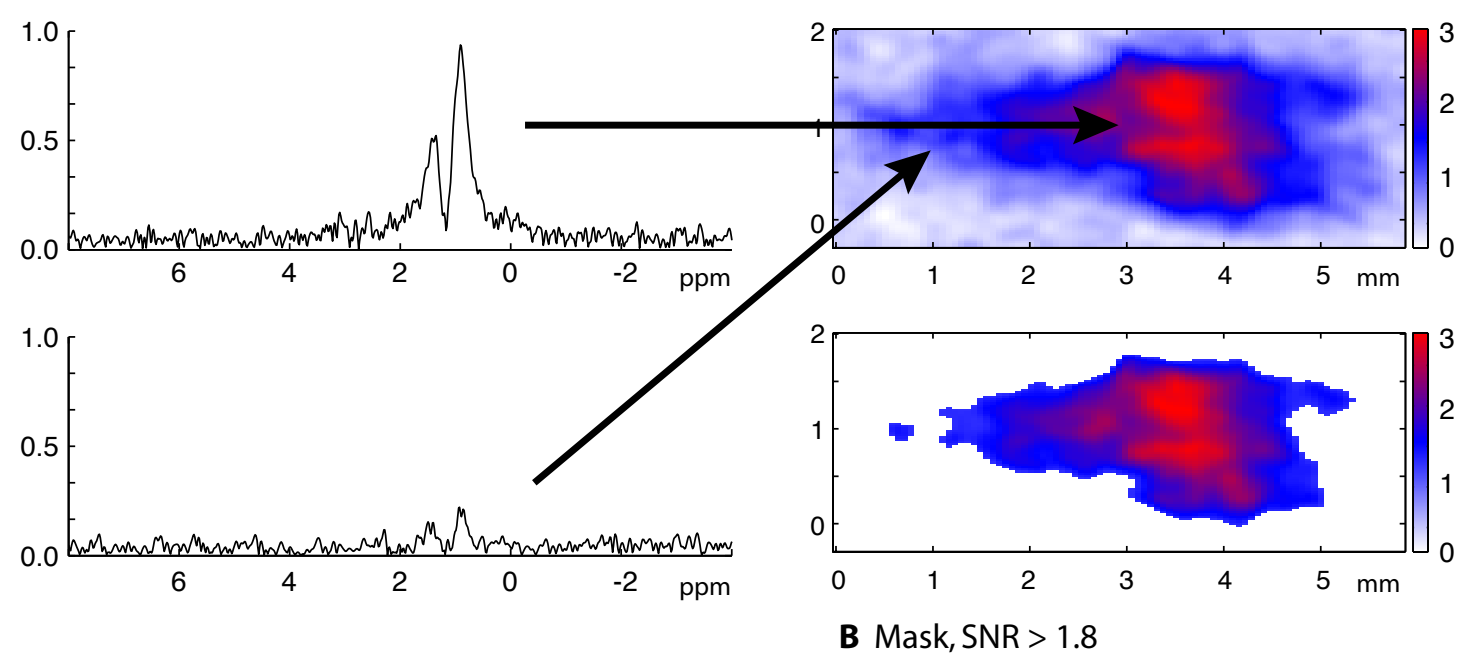

Figure 5.2: SNR thresholding of the image. (A) Comparing spectra from two different regions in the catalyst bed. (B) Masking the image by requiring that $\mathrm{SNR}>1.8$. The masking is primarily used to avoid fitting spectra dominated by noise. If the SNR is too low, the fitting algorithm becomes time consuming and inaccurate.

which is fit to the spectrum. The specific Matlab code is given in sec. B.2.2.

It turns out that there is a considerable amount of phase variation across the catalyst bed. Allowing the nlinfit algorithm to work with complex spectra produces disastrous results, such as complex $\lambda$ and $f$. To avoid this problem, the absolute value spectra were used for fitting the spectrum. Furthermore, The line fitting algorithm fails when the SNR approaches unity. In such cases the algorithm attempts to fit the model spectrum to the noise. This not only leads to bad values for the line width and other parameters, but it requires a long time for the algorithm to converge, and, in fact, often fails to converge when the $\mathrm{SNR} \lesssim 1.6$. To avoid wasting computational time fitting noise, the region of interest is chosen via thresholding. The SNR is calculated for the spectrum of each voxel and only those voxels with SNR> 1.8 are used for for line fitting. This is depicted in fig. 5.2. 


\subsection{Results and Discussion}

Figure 5.3 shows the line width of propane in a bed of silica gel for temperatures ranging from $40^{\circ} \mathrm{C}$ to $140^{\circ} \mathrm{C}$. As expected, the line widths are narrower for higher temperatures due to motional averaging of the susceptibility induced field gradients. The correlation is linear to within $0.3 \%$

$$
L W(T)=69.6 \pm 0.6 \mathrm{~Hz}-T\left(0.200 \pm 0.006 \mathrm{~Hz} /{ }^{\circ} \mathrm{C}\right)
$$

While this approach appears promising for the relative temperature variations, it produces unrealistically large absolute temperatures on the order of $300^{\circ} \mathrm{C}$. The temperature in the catalyst bed was $<160^{\circ} \mathrm{C}$ and the linedwidths are on the order of $30 \mathrm{~Hz}$, compared to the $50 \mathrm{~Hz}$ line width at $100^{\circ} \mathrm{C}$ observed in the calibration experiment. This discrepancy is likely due to the fact that the calibration has been performed on a considerably different system from the imaging experiments. Lacking other calibration data, we propose that the relative change in line width with temperature is, at the very least, an approximation to our microreactor system. Clearly, this needs to be validated both with thermocouple measurements and with a line width calibration using the micro-reactor itself.

The spatial variation of the parameters obtained from fitting the spectrum is shown in fig. 5.4. Of primary importance is the fact that the spatial variation of the line width (fig. 5.4D), is not correlated with the spatial map of $B_{0}$ inhomogeneities (fig. $5.4 \mathrm{~A}$ ). In other words, the line width variation is not due simply to local inhomogeneities in the main magnetic field, but rather reflects the extent of motional averaging at that position.

The final map of temperature variation is obtained from the line width map and 


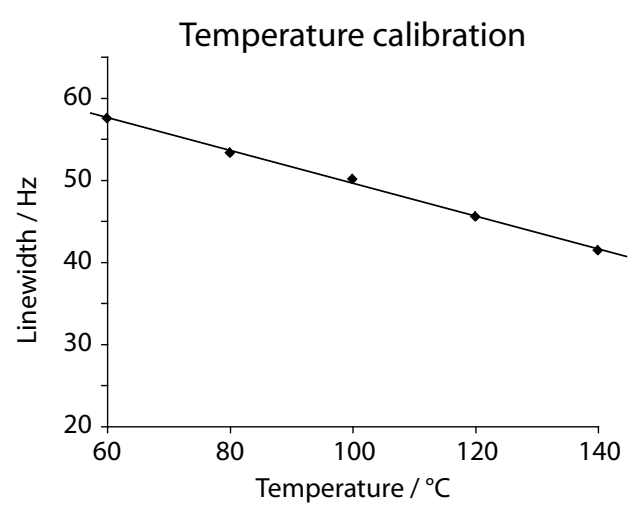

Figure 5.3: line width vs. temperature for propane gas in 60-200 mesh silica gel. The temperature dependence is linear over the range of temperatures used in the experiments $\left(60-140{ }^{\circ} \mathrm{C}\right), L W=T(-0.200 \pm 0.006) \mathrm{Hz} /{ }^{\circ} \mathrm{C}+(69.6 \pm 0.6) \mathrm{Hz}$.

the temperature calibration and is shown in fig. 5.5. The magnitude of the temperature variations presented here should be taken as approximate, given the discrepancies in the temperature calibration discussed above. The trend, however, is clear; given that motional averaging at higher temperatures leads to narrower line widths, we see the expected temperature drop across the catalyst bed as the gas, preheated in the heat exchange coil, loses heat to the surrounding catalyst bed as it flows through.

Another parameter of interest is the apparent peak separation (fig. 5.4B). This is calculated by taking the frequency difference between the peak tops. If the spectrum was pure thermally-polarized propane, then this apparent separation would decrease with increasing line width, due to the overlap of the tails with the adjacent peak. However, the apparent peak separation is not correlated with the line width (fig. 5.4C), and does not appear to be strongly correlated with the offset (fig. 5.4A). This further supports the need for a mixture of PASADENA and thermal signal to explain the spectrum, despite the lack of unambiguous, large antiphase peaks. 
A Offset due to local $\Delta \mathrm{B}_{0}$

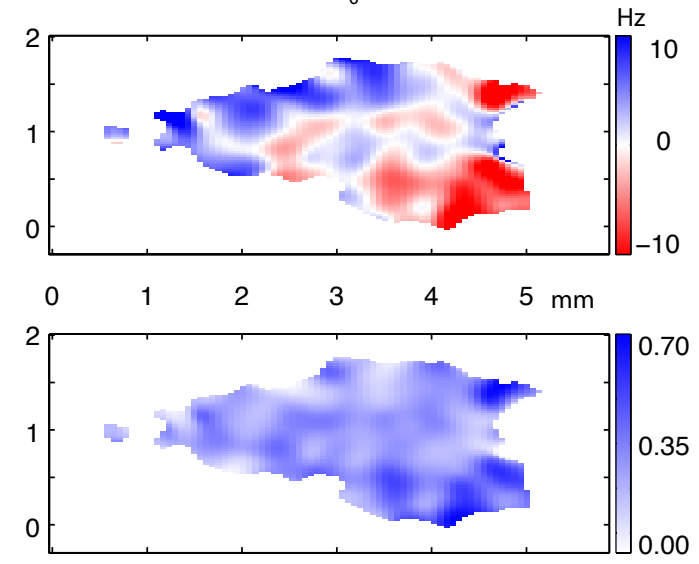

C \% PASADENA lineshape
B Apparent peak separation
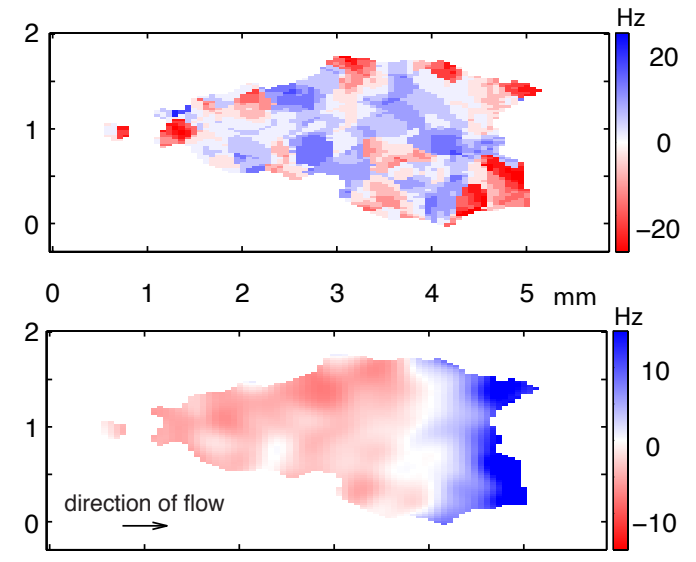

D Linewidth variation

Figure 5.4: Spatial variation of the spectral parameters from the fitting algorithm. (A) Position of the spectrum, reflecting $B_{0}$ inhomogeneities within the catalyst bed. (B) Apparent peak-to-peak distance, taken from the peak heights. (C) Fraction of signal intensity that comes from the PASADENA spectrum. (D) Peak line width. Note that (B) is taken from the spectrum directly, rather than the fit, resulting in lower resolution of the magnitude scale. If the spectrum was purely thermal propane, the peak separation would track the line width due to overlap of each peak with the broadened tail of the adjacent peak. Furthermore, the line width variation is not due to the local inhomogeneities in the $B_{0}$ field as (A) and (D) are clearly not correlated.

A potentially useful feature is the spatial variation of the fraction of PASADENA signal (fig. 5.4C). A large fraction of the signal is from thermally-polarized propane and this can result from hydrogenation by dispersed Rh metal formed during the activation of the catalyst bed. Because the fitting algorithm returns the relative amounts of thermal and PASADENA signals, this has the potential of differentiating which regions of the catalyst bed have catalytically active reduced metal, and which regions of the catalyst bed have active supported catalyst. It is interesting to note that the variation in fig. $5.4 \mathrm{C}$ appears to be correlated with the field inhomogeneity (fig. 5.4). One reason for this could be a significant difference in the susceptibility of the supported and reduced catalyst due to the 


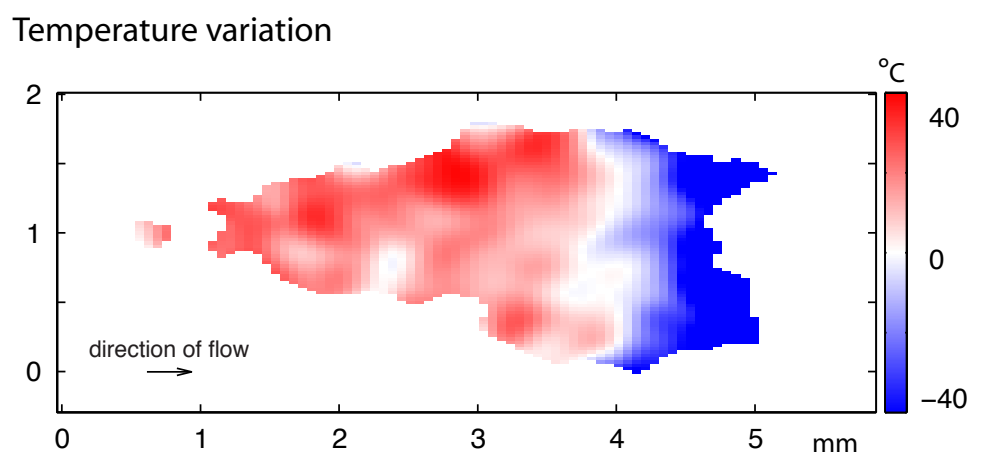

Figure 5.5: The temperature variation within the catalyst bed is calculated from the line width map, using the calibration data from fig. 5.3.

different oxidation states of the Rh.

In summary, the results presented here demonstrate the potential that $p-\mathrm{H}_{2}$ induced polarization has for MRI based thermometry in micro-reactors, as well as for characterizing the extent to which the supported catalyst has been reduced. However, to make this approach quantitative, a number of important control experiments need to be performed. First, the extent of the signal arising from $p-\mathrm{H}_{2}$ polarization can be determined by running control experiments using $n-\mathrm{H}_{2}$. Furthermore, clever use of the imaging gradients can be used to purge the thermal signal arising from relaxation upstream on the field of view or from the reduced catalyst. There are multiple ways to do this, but one simple approach [3] exploits the fact that the PASADENA signal is a mixture of zero and double quantum coherences. This allows specific coherences to be filtered with the use of gradient pulses to dephase the thermal signal (single quantum coherences) while selectively rephasing the double quantum coherence of the PASADENA signal. Furthermore, the temperature measurements can be validated by placing thermocouples at the inlet and the outlet of the catalyst bed. Finally, the line width calibration needs to be carried out using the micro- 
reactor itself. The first stage of planning for the next round of experiments using these improved controls is finished and the experiments will be carried out soon. 


\section{Chapter 6}

\section{Microscopic Pressure Maps in a}

\section{Catalyst Bed}

\subsection{Introduction}

As introduced in ch. 2 and 4, many important chemical reactions take place in heterogeneous environments, such as fluids flowing through a packed-bed reactor where they react at the solid-fluid interface. The optimization of such packed bed reactors is an important problem in chemical engineering $[92,93]$. Characterizing heat and mass transport throughout the reactor is particularly important in the design and optimization of packedbed reactors.

The spatial dependence of parameters, such as pressure and velocity of the flowing gas, can be measured by inserting probes directly into the reactor. This is routine in the characterization of full-scale (industrial) and lab scale reactors [20], but has significant 
consequences when characterizing micro-reactors. The problem is one of scale; the size of the probes is larger than the length scale of the heterogeneity in the catalyst bed. In other words, the measurements become invasive, perturbing the properties that are being measured.

We present a method for non-invasively obtaining microscopic measurements of gas pressure within a packed-bed catalytic micro-reactor, based on MRI. The maps are derived from MRI velocimetry measurements of polarized gas in a catalyst bed [34] (sec. 2). This is done by relating pressure to the flow map using a Navier-Stokes equation which is averaged over the microstructure of the packed-bed reactor. Our approach provides noninvasive pressure measurements at sub-millimeter spatial resolutions. It should be useful for studying the spatio-temporal aspect of chemical reactions taking place in random media, such as heterogeneous catalysis.

\subsection{Methods}

\subsubsection{Navier-Stokes Equation}

The steady state Navier-Stokes equation for compressible fluids is:

$$
\rho \vec{v} \cdot \nabla \vec{v}=-\nabla p+\eta \Delta \vec{v}+\left(\frac{1}{3} \eta+\zeta\right) \nabla(\nabla \cdot \vec{v})
$$

where $p$ is the pressure, $\vec{v}$ is the fluid velocity, $\rho$ is the density, $\eta$ is the viscosity and $\zeta$ is the second viscosity. In general, the coefficients $\rho, \eta$ and $\zeta$ are functions of some spatial coordinate, $\vec{r}$. The partial differential equation (PDE) is subjected to appropriate boundary conditions. 
Typical applications of the Navier-Stokes equation use macroscopic pressure boundary conditions and solve for the resulting steady state velocity field in some interior region of interest. The situation is quite different for the experimental setup described in ch. 4 . From a theoretical point of view, the situation is challenging due to the heterogeneous nature of the catalyst bed. This creates a complicated, random set of boundary conditions at the microscopic level. Luckily, we are not interested in calculating the velocity field from such complicated, unknown boundary conditions. Instead, we have a measurement of the steady state velocity field and wish to extract the microscopic pressure variations due to the heterogeneities in the catalyst bed. Given that we are dealing with a highly compressible fluid in a random packed catalyst bed, we expect the system to exhibit interesting pressure fluctuations. In order to solve for the desired pressure map, we transform this equation into something that is better conditioned. The approach, and some issues to consider, are presented below. This is followed by a discussion of the numerical techniques we have pursued to perform this numerical calculation.

\subsubsection{Transformation to the Poisson Equation}

If the coefficients of eq. 6.1 are constants with respect to the spatial coordinate, $\vec{r}$, the divergence of each side yields a scalar equation:

$$
\nabla^{2} p=-\rho[\nabla \vec{v}: \nabla \vec{v}+\vec{v} \cdot \nabla \nabla \cdot \vec{v}]+\left(\frac{4}{3} \eta+\zeta\right) \nabla^{2}(\nabla \cdot \vec{v})
$$

where the symbol ':' denotes a double-contraction and $\nabla \nabla$ is a dyadic product. This is a Poisson equation with the source term given by the expression on the right hand side. This equation can be solved for the pressure field, $p(\vec{r})$, by imposing boundary conditions and 
Length scales in the catalyst bed

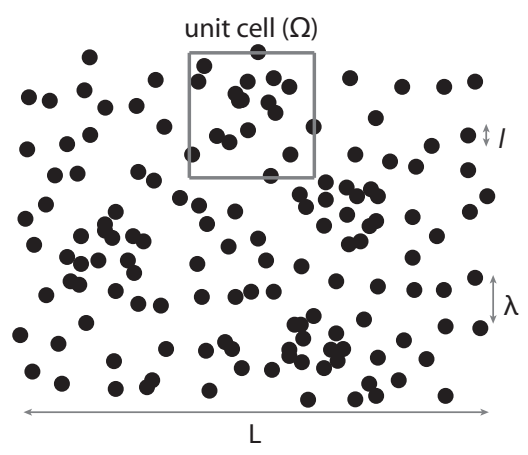

Figure 6.1: The solid catalyst grain and its random packing are shown as a distribution of black discs. The unit cell, $\Omega$, contains several catalyst grain particles, but is smaller than the reactor bed. $l$ is the length scale of the catalyst grain, $\lambda$ is the scale of density fluctuations in the catalyst packing and $L$ is the macroscopic scale of the reactor bed. We require that $l \ll \lambda \ll L$.

using standard methods to invert the Laplacian operator.

However, in a packed-bed reactor, these coefficients are not constant, but are functions of space with variations on the length scale of the catalyst grain. The approach above does not appear to be applicable. On the other hand, a simple flow model, such as eq. 6.2, is desired. We claim that the volume averaged Navier-Stokes equation can be transformed into eq. 6.2. Specifically, we claim that such a Poisson equation holds if we average over the catalyst grain and the following two conditions are met:

1. The volumes, $\Omega(\vec{r})$, used for averaging are large compared to the size of the catalyst grain. In the MRI experiment, we take $\Omega$ to be the size of an imaging voxel.

2. $\Omega(\vec{r})$ is smaller than the size of the reactor bed, so that mesoscopic heterogeneities across the bed can be depicted.

Figure 6.1 illustrates the requirements for averaging. The length scale of the 
catalyst grain is $l$. The heterogeneities in packing density, flow velocity and pressure occur on the $\lambda$ scale. The macroscopic scale of the reactor bed and channel is $L$. The volume averaging of eq. 6.2 is performed over $l$, but the variations on the $\lambda$ length scale remain.

The experimental details concerning the preparation of the micro-reactor, the flow setup and the pulse program are described in ch. 4, as well as sec. A.2 and B.1. The important details for this discussion are the size of the silica gel particles, 200-400 mesh (i.e particle diameters of $37-74 \mu \mathrm{m}$ ), and the voxel size, $170 \mu \mathrm{m} \times 240 \mu \mathrm{m}$. Furthermore, the voxels are already averaged over heterogeneities on a macroscopic length scale because the imaging sequence produces a $2 \mathrm{D}$ projection along the $y$ axis. In summary, each imaging voxel contains at least several grains. Even if this condition were not completely fulfilled, the averaged description of the medium would likely still apply due to the porous nature of silica gel.

\subsubsection{Numerical Techniques}

The two dimensional, component form of eq. 6.2 is given below. Note that the derivatives along $y$ have been dropped due to the averaging across this dimension discussed above.

$$
\begin{aligned}
\sum_{i=x, z} \partial_{i}^{2} P= & \sum_{\substack{i=x, z \\
j=x, z}}\left\{-\rho\left[\left(\partial_{i} v_{j}\right)\left(\partial_{j} v_{i}\right)+v_{j} \partial_{i} \partial_{j} v_{i}\right]+\left(\frac{4}{3} \eta+\zeta\right) \partial_{i}^{2} \partial_{j} v_{i}\right\} \\
P_{x x}+P_{y y}= & -\rho\left(v_{x}^{(x)}+2 v_{x}^{(z)} v_{z}^{(x)}+v_{z}^{(z)}+v^{(x)} v_{x x}^{(x)}+v^{(x)} v_{x z}^{(z)}+v^{(z)} v_{z x}^{(x)}+v^{(z)} v_{z z}^{(z)}\right) \\
& +\left(\frac{4}{3} \eta+\zeta\right)\left(v_{x x x}^{(x)}+v_{z z x}^{(x)}+v_{x x z}^{(z)}+v_{z z z}^{(z)}\right)
\end{aligned}
$$




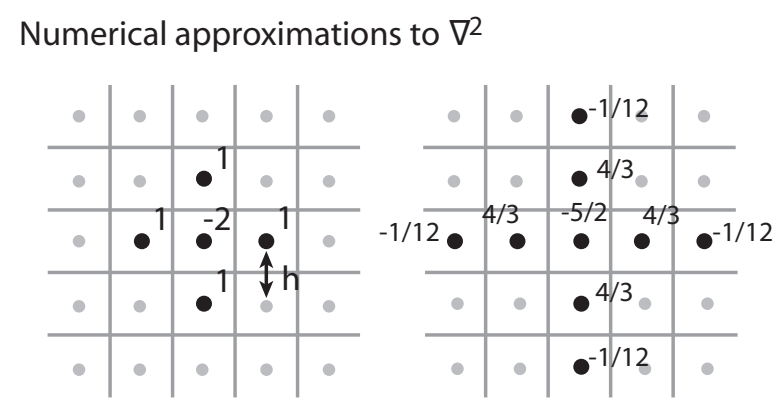

Figure 6.2: Examples of three point and five point stencils in $2 \mathrm{D}$ for the $\nabla^{2}$ operator.

The velocity field obtained from MRI velocimetry is in the form of a $2 \mathrm{D}$ grid (sec. 4.2). Therefore, a grid-based numerical approach is appropriate for solving eq. 6.3.

Grid-based numerical techniques approximate the derivatives with finite difference equations $[53,143]$. Three point centered differences are often used, but we chose to use five and seven point centered differences for improved accuracy [2]. The relevant approximations needed by eq. 6.3 are,

$$
\begin{aligned}
f^{\prime}(x)= & \frac{-f(x+2 h)+8 f(x+h)-8 f(x-h)+f(x-2 h)}{12 h}+O\left(h^{4}\right) \\
f^{\prime \prime}(x)= & \frac{-f(x+2 h)+16 f(x+h)-30 f(x)+16 f(x-h)-f(x-2 h)}{12 h^{2}}+O\left(h^{4}\right) \\
f^{\prime \prime \prime}(x)= & \frac{f(x+3 h)-8 f(x+2 h)+13 f(x+h)-13 f(x-h)+8 f(x-2 h)-f(x-3 h)}{8 h^{3}} \\
& +O\left(h^{4}\right)
\end{aligned}
$$

where $\mathrm{h}$ is the distance between voxels. It is the third derivatives in eq. 6.3 , that requires a seven point centered difference to ensure that all the errors are $O\left(h^{4}\right)$. A schematic of the Laplacian, $\nabla^{2} f$, using three and five point centered differences is given in fig. 6.2.

When applying the numerical derivatives, special care must be taken at the boundaries to ensure that the derivatives are defined. An example boundary is given in fig. 6.4, 


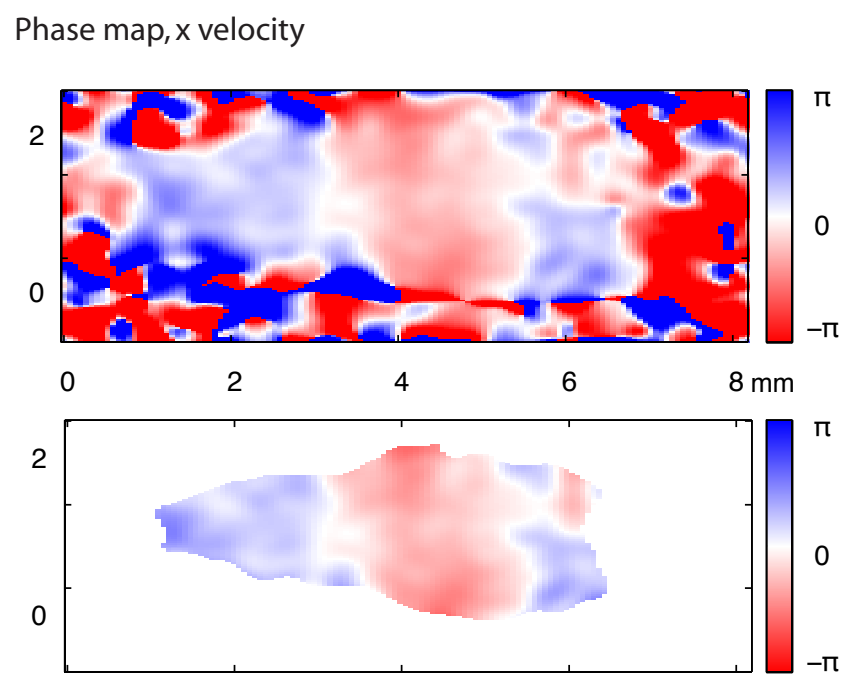

Figure 6.3: Thresholding the phase difference map for the $x$ component of velocity. Note the severe phase distortions in the noise region surrounding the catalyst bed (compare with the SNR map, fig. 5.2).

showing points that are ill-defined for the numerical derivatives. A discussion of the thresholding used to define this region is discussed in sec. 5.2. The signal outside of the thresholded region is dominated by noise and is thus masked out to prevent interference with the calculation. The phase wrapping in the noise region is shown in fig. 6.3. The simplest approach to calculating the numerical derivatives is to simply ignore the points too close to the boundary. This has the effect of shrinking the interior regions slightly, as depicted in fig. 6.4. This effect can be lessened somewhat by using three point centered differences [2] near the boundary instead of five (or seven) point centered differences.

In this case, we know the macroscopic boundary conditions, i.e. the inlet and outlet pressures, but not the actual values at the boundary defined by the thresholding described above. We estimate these boundary values using the bulk pressure drop. We know that the macroscopic pressure difference results in the bulk velocity through the catalyst bed 
Boundary losses

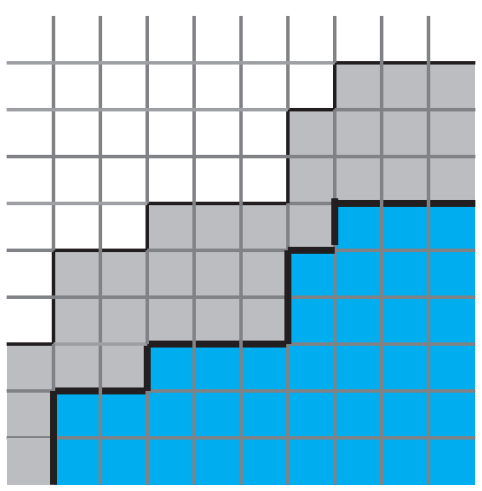

Figure 6.4: In order for the numerical derivative to be well defined, the data points near the edges of the boundary must be neglected (grey), effectively shrinking the interior region (blue) by several pixels.

(Darcy's Law [141]). The heterogeneity that appears in the measured velocity map will be due to perturbations of an otherwise linear pressure drop. We expect the linear pressure drop to be a good first order approximation. As shown below, the perturbations to the linear pressure gradient are actually quite small relative to the magnitude of the pressure and the pressure drop, justifying this assumption.

\section{Iterative Approaches}

The most common approach to solving the Laplace equation is to use the numerical definition of the derivatives to set up a recurrence relation for the pressure $[53,143]$. This is easily adapted to the Poisson equation by simply adding in the source term. For example, the simplest form of this recursive approach is Jacobi iteration. Using the five point centered 
difference for the Laplacian operator, we get

$$
\begin{aligned}
\nabla^{2} P(x, z)= & \frac{-P(x+2 h, z)+16 P(x+h, z)-30 P(x, z)+16 P(x-h, z)-P(x-2 h, z)}{12 h^{2}} \\
& +\frac{-P(x, z+2 h)+16 P(x, z+h)-30 P(x, z)+16 P(x, z-h)-P(x, z-2 h)}{12 h^{2}} \\
= & -\frac{60}{12 h^{2}}\left\{P(x, z)-\frac{1}{60}[-P(x+2 h, z)+16 P(x+h, z)\right. \\
& +16 P(x-h, z)-P(x-2 h, z)-P(x, z+2 h)+16 P(x, z+h) \\
& +16 P(x, z-h)-P(x, z-2 h)]\} \\
= & -\frac{5}{h^{2}}[P(x, z)-\langle P(x, z)\rangle]
\end{aligned}
$$

Note that the exact form of the weighted average, $\langle P(x, z)\rangle$, depends only on the order of centered difference used in the approximation. The recurrence relation is formed by computing the weighted average on an initial guess of $P(x, z)$, allowing a better estimate of $P(x, z)$. Explicitly, we have for the Poisson equation,

$$
\begin{aligned}
\nabla^{2} P(x, z) & =f(x, z) \\
-\frac{5}{h^{2}}[P(x, z)-\langle P(x, z)\rangle] & =f(x, z) \\
P^{n+1}(x, z) & =\left\langle P^{n}(x, z)\right\rangle-\frac{h^{2}}{5} f(x, z)
\end{aligned}
$$

Unfortunately, the Jacobi method converges rather slowly. A faster implementation, the Gauss-Seidel method [53,143], exploits the fact that we compute the new $P(x, z)$ point by point in a grid. If we perform these calculations in a raster across the grid, we can 
use newly calculated values of $P(x, z)$ for half of the points used in $\langle P(x, z)\rangle$,

$$
\begin{aligned}
\left\langle P^{n}(x, z)\right\rangle= & \frac{1}{60}\left[-P^{n+1}(x+2 h, z)+16 P^{n+1}(x+h, z)+16 P^{n}(x-h, z)-P^{n}(x-2 h, z)\right. \\
& \left.-P^{n+1}(x, z+2 h)+16 P^{n+1}(x, z+h)+16 P^{n}(x, z-h)-P^{n}(x, z-2 h)\right]
\end{aligned}
$$

The Gauss-Seidel method is further improved [18] by including the center point in the weighted average of eq. 6.9 ,

$$
P^{n+1}(x, z)=(1-\omega) P^{n}(x, z)+\omega\left[\left\langle P^{n}(x, z)\right\rangle-\frac{h^{2}}{5} f(x, z)\right]
$$

When $\omega=1$, this is identical to the Gauss-Seidel method. When $0<\omega<1$, the technique is called successive under-relaxation and can help an otherwise divergent iterative scheme to converge on a solution. When $1<\omega<2$, the technique is called successive over-relaxation and can greatly speed up the convergence, but can also lead to divergence if $\omega$ is chosen to be too large. There is no way of determining ahead of time what value of $\omega$ leads to fastest convergence. [18]

\section{Other Approaches}

Non-iterative methods exist for inverting the $\nabla^{2}$ operator. We have explored two such techniques. These approaches are appealing in that they are analytical solutions and do not have the potential problems of accumulated round off error and sensitivity to the initial guess that can be problematic in iterative approaches. However, they each present

difficulties of their own. In light of these difficulties, we do not yet have final results using these techniques; however, they are being actively pursued. 
The most common alternative to iterative solutions involving the Laplacian operator is a simple matrix inversion [53]. The basic approach is to arrange the source term and the boundary conditions into an array. The $\nabla^{2}$ operator can then be represented as a matrix and inverted to obtain $P(x, z)$ :

$$
\begin{aligned}
\nabla^{2} P(x, z) & =f(x, z) \\
\mathbf{D} \vec{P}+\vec{B} & =\vec{f} \\
\vec{P} & =(\mathbf{D})^{-1}(\vec{f}-\vec{B})
\end{aligned}
$$

where $\vec{f}$ is the source term, $\vec{B}$ contains the pressure values at the edges of the boundary where $\nabla^{2}$ gets cut off and $\mathbf{D}$ is the matrix representation of the $\nabla^{2}$ operator on the interior.

For a simple rectangular region, the $\nabla^{2}$ matrix takes on a sparse, banded structure with a condition number of 1 . The unusual shape of our region causes some difficulties and ambiguities in how to arrange the data into vectors. At this point, we do not have a representation for $\nabla^{2}$ with a good condition number. This can be addressed by throwing out some of the known data to get a better behaved boundary (e.g. a rectangle). The real problem, however, is caused by the large size of the $\nabla^{2}$ matrix, even if it is well conditioned. The velocity data sets have nominal grid sizes of $128 \times 128$. Once rearranged, the pseudovectors have $2^{14}$ entries each, and the $\nabla^{2}$ matrix has $2^{14} \times 2^{14}=2^{28}$ entries. Gaussian elimination would require $2^{84}$ operations to invert this matrix! Algorithms designed to take advantage of sparseness exist, but currently, we are simply limited by the amount of memory that Matlab is able to use. The next step is to implement this algorithm on a computation server that has sufficient memory and parallel processing capabilities.

Another appealing approach is to use the properties of Fourier transforms to invert 
the $\nabla^{2}$ operator [57]. Initially, this looks quite appealing, as the Laplacian operation under Fourier transform is typically given as

$$
\mathcal{F}\left\{\nabla^{2} P\right\}=-|\vec{k}|^{2} \mathcal{F}\{P\}
$$

However, this assumes that the surface terms go to zero. While this is true in many applications, it certainly is not true that the pressure, velocity or derivatives thereof go to zero at the boundary inside the catalyst cell. An explicit derivation that includes the surface terms gives

$$
\mathcal{F}\left\{\nabla^{2} P(\vec{x})\right\}=-|\vec{k}|^{2} \mathcal{F}\{P(\vec{x})\}+\oint_{B} \mathrm{e}^{-\mathrm{i} \vec{k} \cdot \vec{x}}(\nabla \cdot P(\vec{x})+\mathrm{i} P(\vec{x}) \vec{k} \cdot \overrightarrow{1}) \mathrm{d} l
$$

where the line integral is taken over the boundary, $B$, and the $\vec{k} \cdot \overrightarrow{1}$ notation implies a summation over the components of $\vec{k}$. This is easily rearranged to give

$$
P(\vec{x})=f \otimes \mathcal{F}^{-1}\left\{\frac{1}{-|\vec{k}|^{2}}\right\}+\mathcal{F}^{-1}\left\{\frac{\oint_{B} \mathrm{e}^{-\mathrm{i} \vec{k} \cdot \vec{x}}(\nabla \cdot P(\vec{x})+\mathrm{i} P(\vec{x}) \vec{k} \cdot \overrightarrow{1}) \mathrm{d} l}{-|\vec{k}|^{2}}\right\}
$$

While the convolution of the source term is easy to compute, the Fourier transform of the line integral is more complicated to perform in practice. This approach is being actively pursued.

\subsection{Results}

The resulting velocity and pressure maps from a micro-reactor are shown in fig. 6.5. The pressure field (fig. 6.5B) shows the linear drop in pressure across the length of the catalyst bed, along the principal direction of flow. None of the smaller fluctuations can be seen due to their very small amplitudes. To observe these fluctuations, we decompose the 


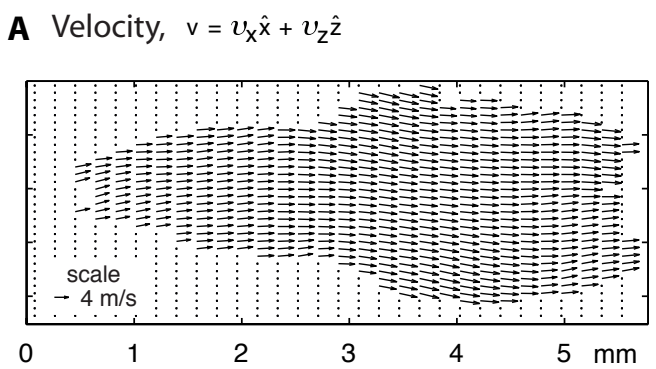

B Bulk Pressure Drop
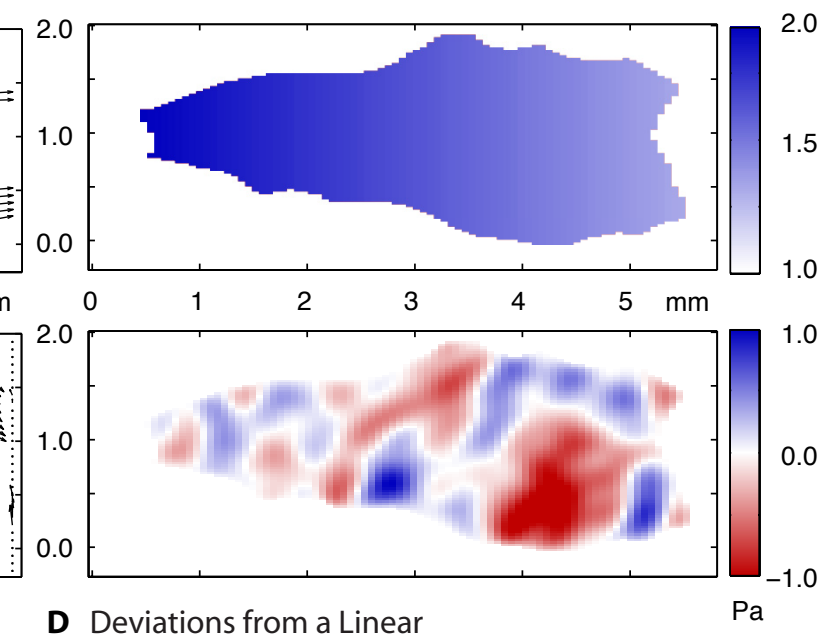

Pressure Gradient

Figure 6.5: Microscopic pressure map calculated from the Poisson equation. (A) Velocity map obtained via MRI (ch. 4). (B) Pressure map obtained via successive overrelaxation. Both (A) and (B) have the expected bulk behavior. (C) Shows the variations in the velocity by subtracting off the average $z$ velocity. (D) Shows the variations in the pressure map by subtracting off the linear pressure gradient.

pressure field into a mean value, $\langle p(\vec{r})\rangle$, and its local variations, $\Delta p(\vec{r})$,

$$
p(\vec{r})=\langle p(\vec{r})\rangle+\Delta p(\vec{r}) .
$$

The mean value is obtained by either averaging over a large enough region centered on each pixel, $\vec{r}$, or by taking the linear pressure drop; both methods give identical results in the present case. By subtracting the mean value from $p$, a map of the local pressure variations is obtained (fig. 6.5D). The mean pressures are on the order of $100 \mathrm{kPa}$, while the microscopic variations are seen to be on the order of Pa.

These results were calculated using successive over-relaxation with $\omega=1.90$ and a variation limit of $0.001 \mathrm{~Pa}$ for determining convergence. The second viscosity, $\zeta$, was assumed to be equal to the dynamic viscosity, $\eta$, as information on the second viscosity 


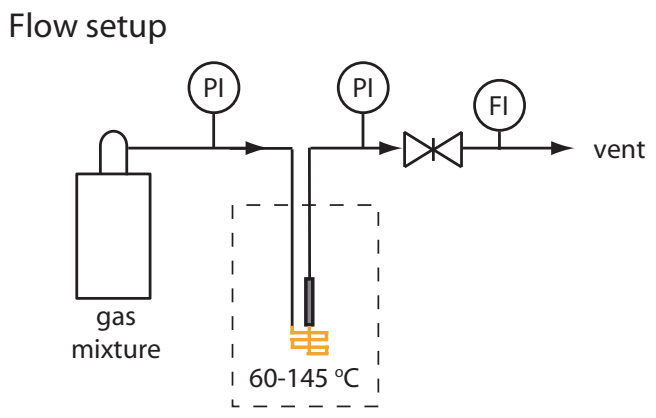

Figure 6.6: Flow setup for micro-reactor imaging. Both the inlet and outlet pressures are monitored.

coefficients is not readily available. The dynamic viscosities were $16.6 \times 10^{-6}, 8.4 \times 10^{-6}$ and $7.4 \times 10^{-6} \mathrm{~Pa}$ s, for $\mathrm{N}_{2}, \mathrm{H}_{2}$ and propylene, respectively. $\eta$ is the weighted average. The mole fractions of the gas mixture were $0.1,0.7$ and 0.2 , respectively. The absolute pressure was 30psig at the inlet, with a 15psi pressure drop across the catalyst cell and a mass flow rate of $\approx 75 \mathrm{sccm}$ at the outlet. The flow setup is depicted in fig. 6.6.

\subsubsection{Convergence}

We have chosen to define convergence in terms of the maximum change in magnitude of the pressure maps between iterations. For example, if the variation limit is set to $0.1 \mathrm{~Pa}$, then the algorithm will consider the solution convergent if the maximum deviation from the previous iteration is $<0.1 \%$, although the average difference is typically much smaller than this. Figure 6.7 compares the relative rates of convergence for the Jacobi, Gauss-Seidel and SOR methods. In all cases, the initial convergence is relatively fast (up to about $0.004 \mathrm{~Pa}$ ), but reducing the relative variation between iterations to below $0.001 \mathrm{~Pa}$ takes the bulk of the time. After trying a range of values for $\omega$, we chose a slightly conservative 


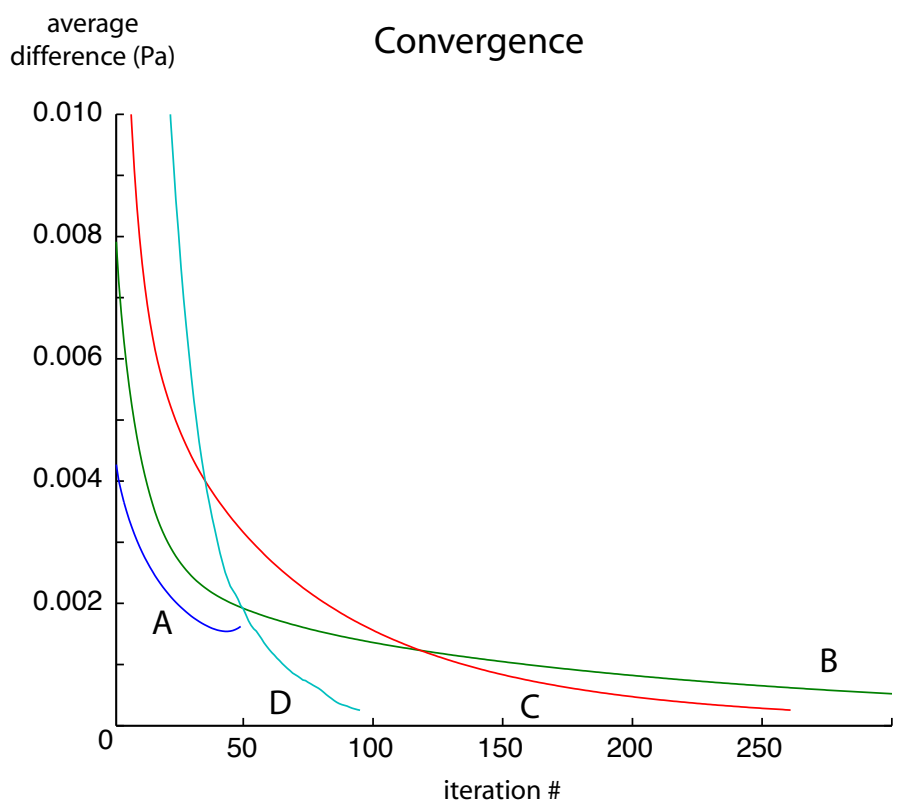

Figure 6.7: Convergence of several iterative schemes. The average deviation between successive solutions vs. the number of iterations, is given for (A) Jacobi iteration, (B) GaussSeidel iteration, (C) and (D) Successive Over-Relaxation (SOR) with $\omega=1.5$ and 1.90, respectively. The Jacobi method begins to diverge after 48 iterations. SOR converges more rapidly as $\omega \rightarrow 1.90$ (Gauss-Seidel is equivalent to SOR with $\omega=1.0$ ), but converges much slower for $\omega=1.95$ and diverges for $\omega=1.99$ (not shown).

value of $\omega=1.90$ to apply to all of the remaining data sets.

\subsubsection{Initial Conditions}

Figure 6.8 shows several different starting conditions: random, sinusoidal, nothing and the source term. Note that all of these starting conditions are taken to be perturbations to the bulk linear pressure drop. If the initial condition is not some variation superimposed on the linear gradient, then the number of iterations required to propagate the boundary conditions into the interior is large enough that significant round off errors accumulate and the real solution is obscured. All of these initial conditions reproduce the same final 
Successive over-relaxation, initial conditions
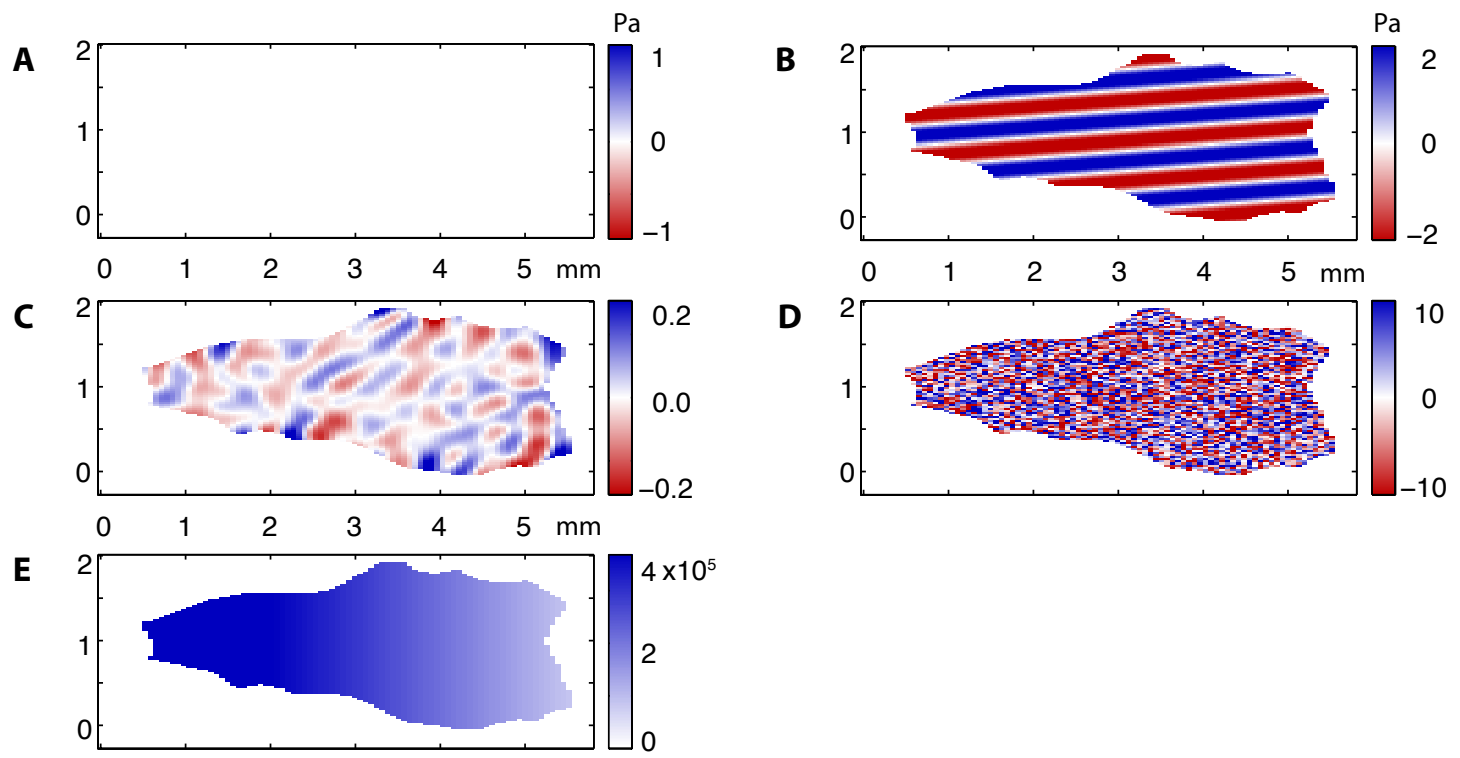

Figure 6.8: Initial guesses for the pressure map. The final pressure map, when defined as the deviation from the linear pressure drop, is robust to the initial conditions; each of these initial conditions produces identical results. (A) No perturbation to the linear drop. (B) Sinusoidal perturbation. (C) Source term of the Poisson equation. (D) Random perturbation. (E) $10 \mathrm{kPa}$ pressure offset and a sharper pressure gradient. Note that all lengths are $\mathrm{mm}$ and all magnitudes are Pa. Also, the actual initial condition is one of these pressure maps added to a linear pressure drop.

pressure variations. Furthermore, if the absolute pressure or the bulk pressure drop is changed (fig. 6.8E), the resulting difference pressure map remains the same, even though the bulk solution is significantly different, reflecting the change in the bulk pressure gradient. This stability with respect to initial conditions indicates that this approach is robust and depends only on the variations in the velocity map, not on the details of the bulk pressure drop used in the calculation. 


\subsection{Discussion}

Our results demonstrate that the hyperpolarized gas-phase MRI measurements presented in ch. 4 provide the basis for an MRI pressure transducer. Numerical solutions of the Navier-Stokes equations, based on the transformation to a Poisson equation, prove to be robust and yield variations in the pressure map on the expected mesoscopic length scale. Knowledge of the pressure and velocity variations within the catalytic cell are important for characterizing and optimizing the reactor. Furthermore, in combination with the temperature maps discussed in ch. 5 and the density maps in ch. 4 , this technique offers the possibility of visualizing the spatial distribution of chemical potentials.

Other accounts of deriving the local pressure variations from MRI velocity maps are found in the medical imaging literature. Tyszka, et. al. [148], provide a brief survey of the approaches taken and the interest in calculating blood pressure gradients during pulsatile blood flow, typically in the aortic arch. Early methods used Bernoulli equations [139], while Yang, et. al. present a method very similar to our own [159]. Yang's approach uses only macroscopic boundary conditions and neglects the viscosity terms, the emphasis being pulsatile flow as opposed to issues encountered with flow through a porous medium. Urchuk [149] uses a volume averaged equation, but invokes the elastic properties of the blood vessels to satisfy the boundary conditions and neglects the convective terms; again, quite a different scenario than our situation. Tyszka, et. al. [148] are also focused on the time resolution of pulsatile flow, using volumetric imaging and a simple iterative scheme that neglects the convective and viscosity terms. In summary, all of these approaches assume that the fluid is incompressible, they neglect the viscosity terms, and they neglect some, or 
all, or the convective terms. Because we are dealing with a gas at elevated temperatures in a porous medium, we cannot assume incompressibility and we expect the viscosity and convective terms to contribute. 


\section{Appendix A}

\section{Experimental Setup}

A great deal of my time and effort in the lab was spent designing, building and optimizing the experimental setups used to perform the experiments described above. The following sections provide the more technical details of the $p-\mathrm{H}_{2}$ flow setups that I have built.

\section{A.1 Parahydrogen Production}

Section 1.2.2 explains how the fraction of $\mathrm{H}_{2}$ molecules in the $p-\mathrm{H}_{2}$ nuclear spin state is controlled. In summary, the symmetry requirements of quantum mechanics couples the rotational and nuclear spin states together. This coupling is advantageous because the rotational states are much easier to polarize due to the larger energy spacing relative to the nuclear spin state (fig. 1.7). In principle, simply cooling $\mathrm{H}_{2}$ below the characteristic rotational temperature (eq. 1.22), $\Theta_{R} \approx 85 \mathrm{~K}$, results in a increased fraction of $p-\mathrm{H}_{2}$ at equilibrium. However, as pointed out in sec. 1.2.2, the conversion of ortho- to parahydrogen 
is forbidden, resulting in very slow kinetics $[79,100]$. This forbidden transition is actually advantageous once a method is found to catalyze the conversion, as it means the resulting $p-\mathrm{H}_{2}$ can be stored for relatively long periods of time without significant back-conversion to $o-\mathrm{H}_{2}$.

A variety of materials can be used to convert ortho- to parahydrogen $[56,147]$. Typically, a paramagnetic powder is used to break the symmetry of the nuclear spin Hamiltonian when the $\mathrm{H}_{2}$ molecule interacts with the surface; evolution under this Hamiltonian makes the transition allowed and results in a much faster equilibration of the ortho/para ratio [42]. I use a paramagnetic powder, $\mathrm{FeO}(\mathrm{OH})$ (Sigma Aldrich \#371254), for this process. The primary advantage of using this catalyst is that the fast equilibration time allows for the production of a constant flow of $p-\mathrm{H}_{2}$.

\section{A.1.1 Liquid $\mathrm{N}_{2}$ Converter}

The first apparatus that I built produces a constant stream of para-enhanced $\mathrm{H}_{2}$. It uses a liquid $\mathrm{N}_{2}$ dewar accessory from a variable temperature (VT) unit to thermalize the $\mathrm{H}_{2}$ at approximately 77K [91]. Specifically, I use a Cryofab CFL dewar with a modified VT insert. This dewar is convenient because there is a Swagelok union at the bottom of the insert that is inserted into the liquid $\mathrm{N}_{2}$. This union is a convenient place to add an additional loop of tubing containing the ortho/para catalyst. Approximately $5 \mathrm{~g}$ of the $\mathrm{FeO}(\mathrm{OH})$ catalyst is poured into a U-shaped section of $1 / 4$ " $\mathrm{Cu}$ tubing that is connected to the insert stem, as shown in fig. A.1. The catalyst is surrounded by glass-wool plugs at both the inlet and the outlet to hold the catalyst in place. The overall height of the 
$p-\mathrm{H}_{2}$ conversion cell, $77 \mathrm{~K}$

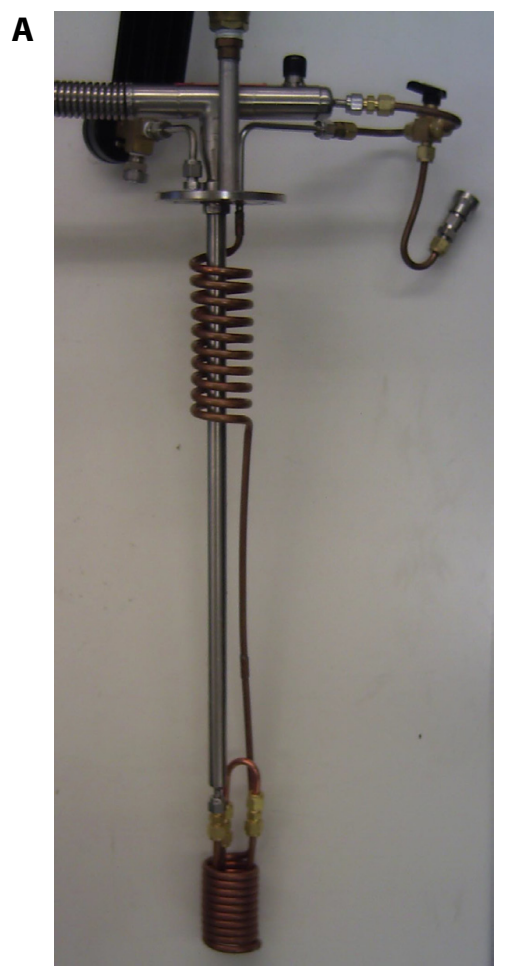

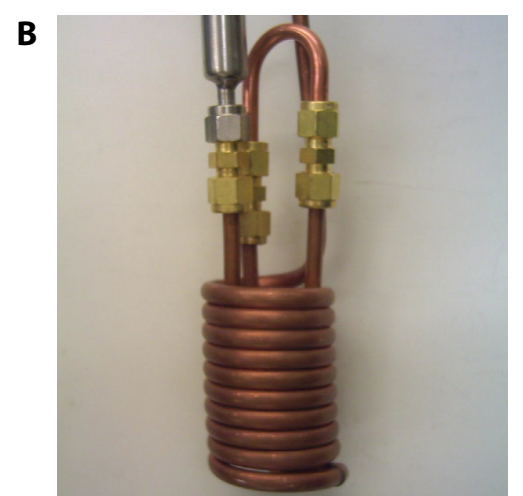

C

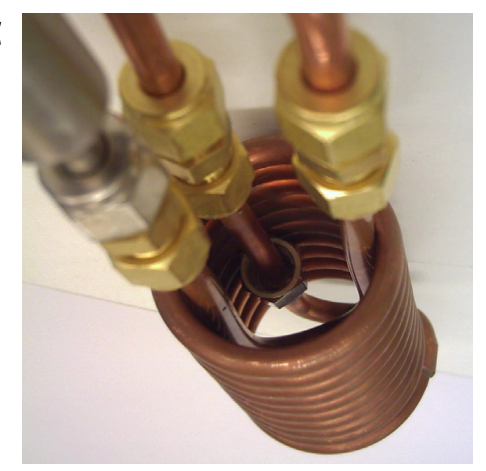

Figure A.1: The heat exchange insert of a VT unit was modified to produce a constant stream of $\mathrm{H}_{2}$ with an enhanced fraction of $p-\mathrm{H}_{2}$. (A) VT insert with two heat exchange coils. (B) and (C) Detail of the modified lower heat exchange coil. A 6" loop of $1 / 4$ " Cu tubing has been added inside the coil. This loop contains the ortho/para catalyst.

additional loop is 6"; the bottom piece, containing the catalyst, is 4" tall. To minimize the chances of $\mathrm{O}_{2}$ or other gases entering the VT insert, a check valve is installed on the outlet of the VT and Swagelok quick-connect unions are used to connect the insert to the main flow manifold. The current catalyst has been in use for approximately two and a half years without a noticeable drop in conversion efficiency.

The VT insert has both a pre-cooling heat exchange coil and a heat exchange coil that is immersed in the liquid $\mathrm{N}_{2}$. This allows efficient cooling of the gas at high flow rates to ensure that the gas has cooled to $77 \mathrm{~K}$ when it reaches the catalyst. This is expected to 


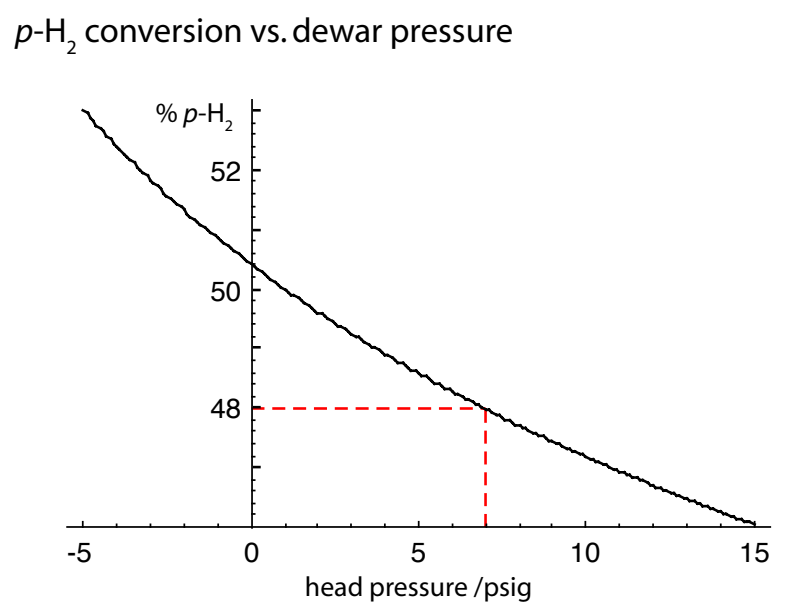

Figure A.2: The boiling point of liquid $\mathrm{N}_{2}$ depends on the head pressure in the dewar. This change in boiling point affects the resulting fraction of $p-\mathrm{H}_{2}$ flowing out of the catalyst cell. The dewar has a check valve with a cracking pressure of $7 \mathrm{psig}$, resulting in $48 \% p-\mathrm{H}_{2}$.

give $\approx 50 \% p-\mathrm{H}_{2}$, as discussed in sec. 1.2.2. The method for testing the actual conversion ratio is described in sec. A.1.3, where it is seen that the best conversion ratio observed is only $48 \% p-\mathrm{H}_{2}$. This results from the boiling point elevation of liquid $\mathrm{N}_{2}$ due to the head pressure of the dewar; the check valve on the safety pressure relief has a cracking pressure of 7psig. The change in boiling point with respect to pressure is calculated using the Clausius-Clapeyron equation [49],

$$
\ln \left(\frac{P}{P_{b}}\right)=\frac{M \Delta H_{v a p}^{b}}{R}\left(\frac{1}{T_{b}}-\frac{1}{T}\right)
$$

where $T$ is the desired temperature, $P$ is the head pressure, $T_{b}$ and $P_{b}$ are the reference temperature and pressure of a known boiling point, $\Delta H_{v a p}^{b}$ is the latent heat of vaporization at $T_{b}$, and $M$ is the molar mass of $\mathrm{N}_{2}$. This is combined with the temperature dependence of the fraction of $p-\mathrm{H}_{2}$ to calculate the expected fraction of $p-\mathrm{H}_{2}$ with respect to the head pressure of the dewar, fig. A.2. A head pressure of 7 psig results in $48 \% p-\mathrm{H}_{2}$, as observed. 


\section{A.1.2 Compressed He Converter}

As discussed in sec. 1.2.2, there is relatively little benefit of using $100 \% \mathrm{H}_{2}$ instead of $50 \% p-\mathrm{H}_{2}$ for sensitivity enhancement; $50 \% p-\mathrm{H}_{2}$ gives a theoretical enhancement of $\approx 2 \times 10^{4}$, whereas $100 \% p-\mathrm{H}_{2}$ only improves this by an additional factor of $3,[36,118]$

(fig. 1.11). Given this, and the relative ease and low cost of of using liquid $\mathrm{N}_{2}$, there is typically little motivation to produce $p-\mathrm{H}_{2}$ of higher than $50 \%$ purity. However, some applications, such as quantum information processing, are concerned with the purity of the nuclear spin state, as opposed to simply the signal magnitude. In these situations, the use of $100 \% p-\mathrm{H}_{2}$ is critical [10]. Several polarization compression experiments were planned, but have not yet been carried out. In preparation for these experiments I designed a setup to produce a stream of $>98 \% p-\mathrm{H}_{2}$.

Figure 1.7 shows that nearly $100 \% p-\mathrm{H}_{2}$ is obtained for temperatures as high as 20K. Liquid He boils at $\approx 4 \mathrm{~K}$ and is readily available, so one can imagine constructing a dewar setup similar to that described above. Unfortunately, $\mathrm{H}_{2}$ freezes at $14.0 \mathrm{~K}$ and liquifies at $20.3 \mathrm{~K}$, creating problems when the goal is to produce a stream of $p-\mathrm{H}_{2}$ gas. Fortunately, commercially available cryostats are available that provide a broad range of cryogenic temperatures. I used a Janis cryostat, model CCS-100, that uses closed cycle ${ }^{3} \mathrm{He}$ refrigeration to provide temperatures ranging from 10K up to room temperature. Figure A.3 shows the cold finger that is placed under vacuum. The first stage is held at $25 \mathrm{~K}$, while the second stage is held at $10 \mathrm{~K}$. A heater and thermocouple on the mounting plate connect to a temperature controller and provide stable control of the sample temperature.

The $\mathrm{H}_{2}$ gas enters the cryostat through $1 / 8$ " stainless steel (SS) tubing. The 


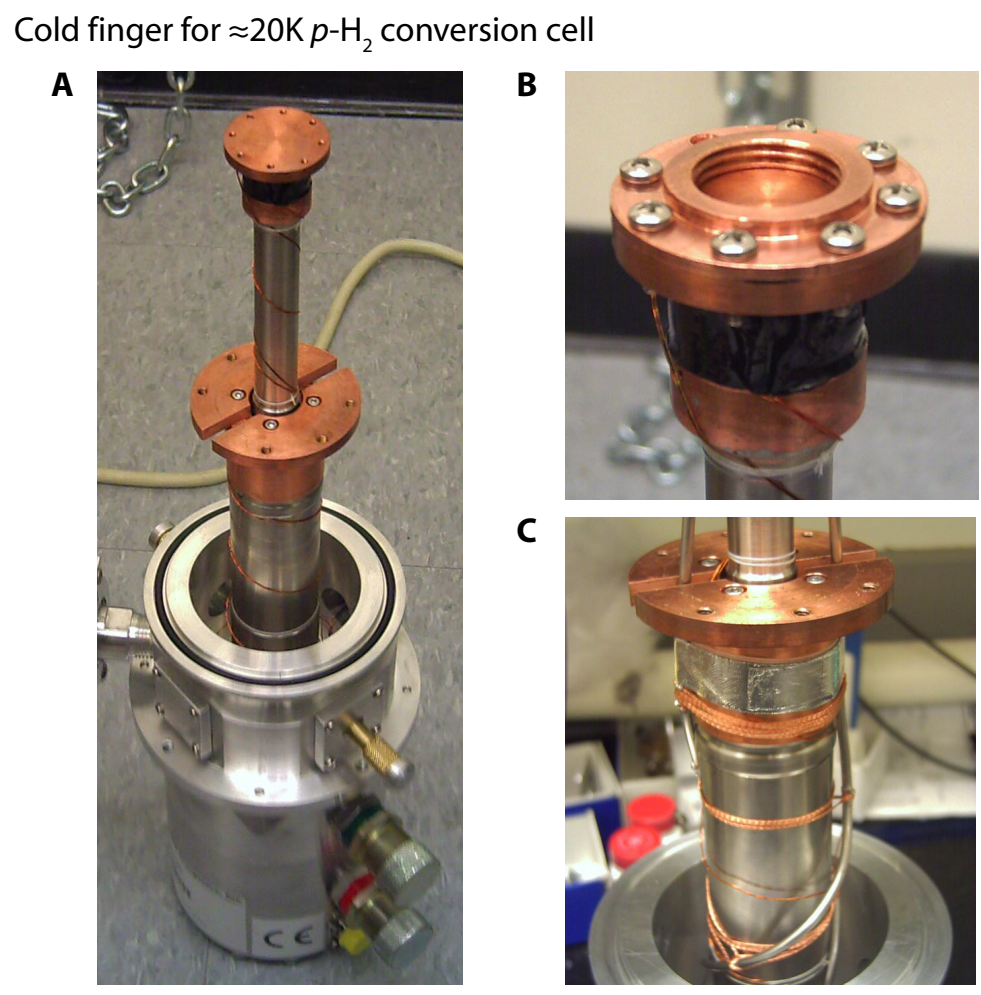

Figure A.3: Janis CCS-100 cryostat adapted for $p-\mathrm{H}_{2}$ production. (A) Cryostat without the vacuum shroud and radiation shield removed, showing the cold finger. (B) Second stage, nominally $10 \mathrm{~K}$, with a mounting plate. The mounting plate has a heater and thermocouple that attach to an external temperature controller. (C) First stage, nominally 25K. The tubing is anchored to this stage to pre-cool the gas and thermally isolate the second stage from the ambient surroundings.

tubing is connected to the first stage to precool the gas and to reduce the heat load on the second stage of the cold finger. SS tubing is used, as it has a smaller thermal conductivity compared to $\mathrm{Cu}$ tubing. $\mathrm{Cu}$ de-soldering braid is used to improve the thermal contact between the SS tubing and the cold finger. Reflective silver tape is used to hold the tubing in place as tightly as possible around the first stage of the cold finger. It is important that the SS tubing not touch the second stage of the cold finger, as this will cause the $\mathrm{H}_{2}$ to freeze, stopping the flow. Instead, the tubing is held onto the mounting plate with reflective 
silver tape and $\mathrm{Cu}$ braid is again used to improve the thermal contact to the mounting plate (see fig. A.3).

The initial design of the catalyst cell was a baffle design. Schematics and images of the final design are shown in figs. A.4-A.12. I machined the cell out of solid Cu stock using a lathe and a milling machine. The first problem with the design was keeping it sealed at cryogenic temperatures. The NPT connections held pressure at room temperature, but not at cryogenic temperatures. This was solved by soldering the NPT connectors to the base. The next problem was the crushed indium seal between the insert and the sheath. The fact that the body and the insert screw together applies a shear force while the indium seal is crushed, this makes it very difficult to get a good seal (it is possible, but the seal fails most of the time). The original plan was to fill the threads with cryogrease, which would freeze and hold the pressure, but simply pumping the cryostat down created enough pressure differential to blow the grease out; I didn't have an easy way to vacuum the inside of the of the cell down at the same rate as the shroud space. A better approach is to have no internal thread, but a number of screws around the edge to bring the insert and sheath together vertically while crushing the indium wire. The problem with this approach is that the sheath walls are too thin; any tapped holes would not be able to withstand the pressure required to crush the indium wire.

The second design kept the adapter plate design for thermal anchoring to the mounting plate. This time, 1/4" $\mathrm{Cu}$ tubing was bent into two U-shapes and soldered into the anchoring base for thermal anchoring (fig. A.13). The Swagelok connectors made sealing the cell simple but the gas didn't have enough time to cool before interacting with 

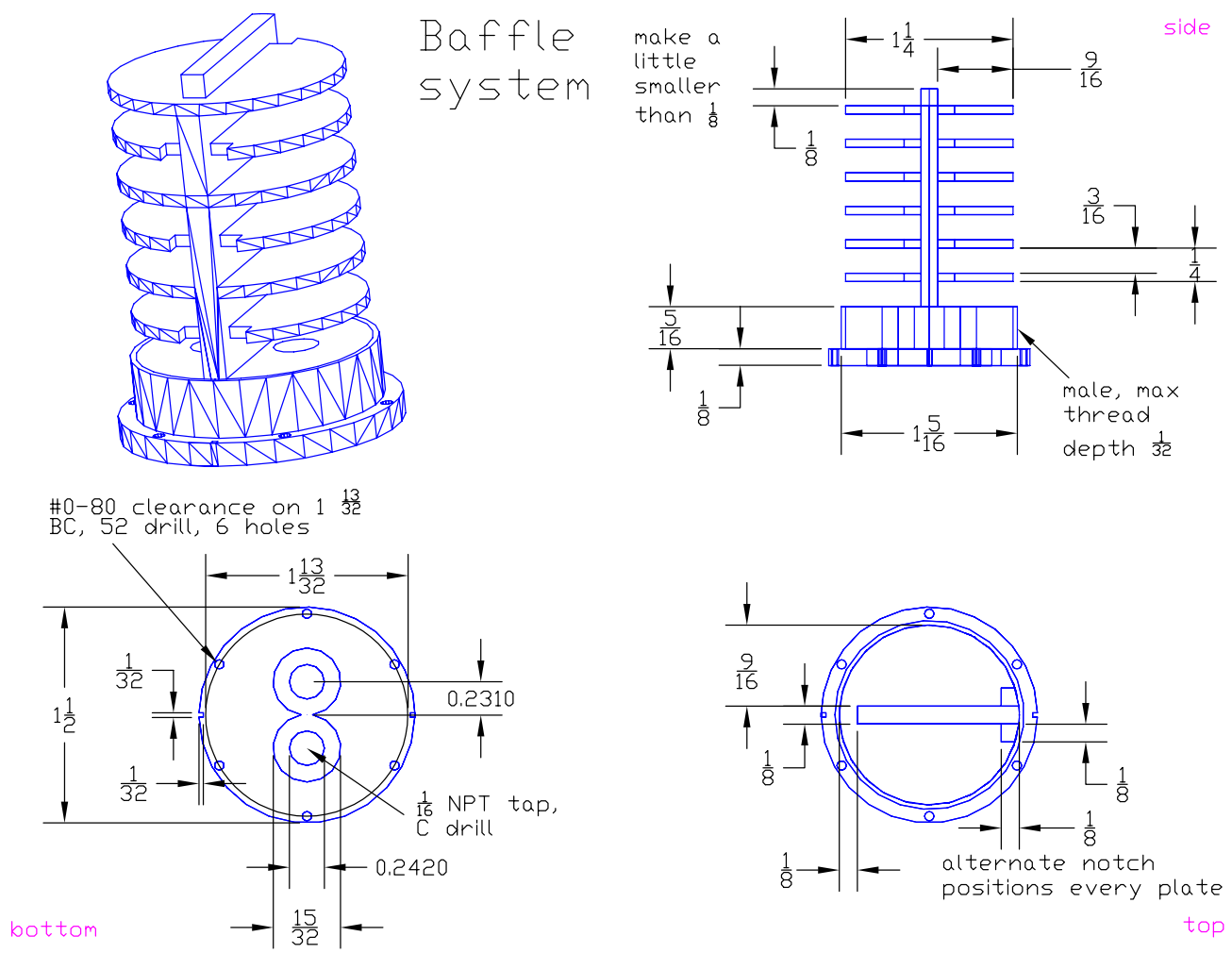

Figure A.4: Schematic of the baffled insert. Note that this design has the perimeter holes for a proper crushed indium wire seal. This was modified when it became clear the tapped threads could not support the force necessary to crush the indium wire. Instead, a left handed thread was placed on the lower part of the insert (see fig. A.12). 
top

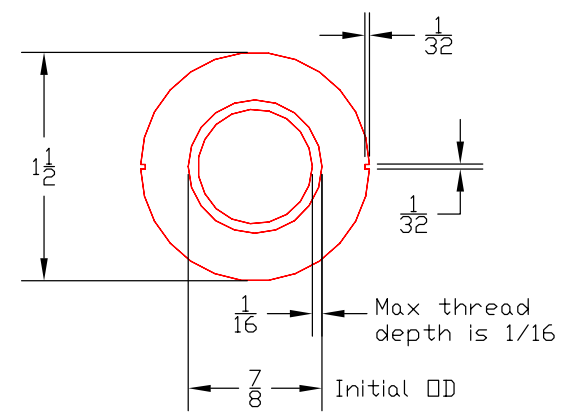

$\# 0-80$ tap on $1 \frac{13}{32} \mathrm{BC}$,

use $\frac{3}{64}$ drill, 6 holes

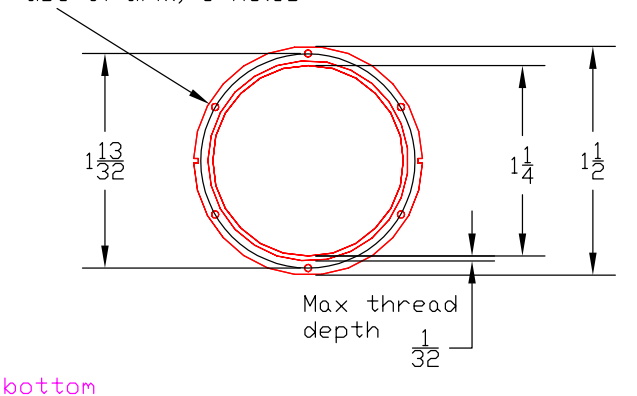

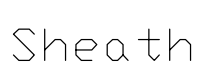
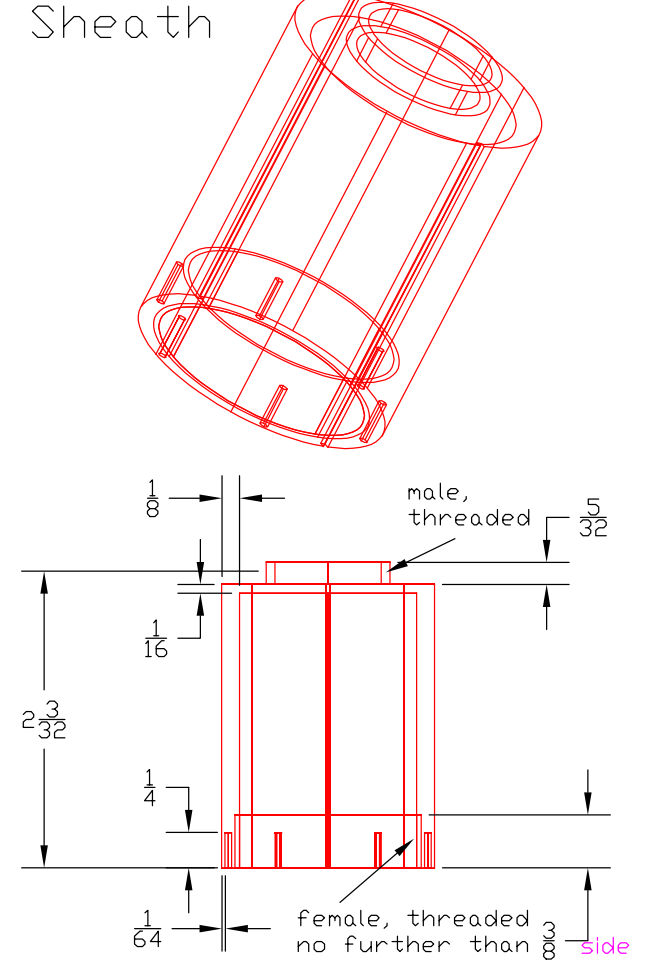

Figure A.5: Schematic of the sheath. As in fig. A.4, this design has the tapped perimeter holes which were removed and replaced by a left handed thread on the inside of the sheath. 

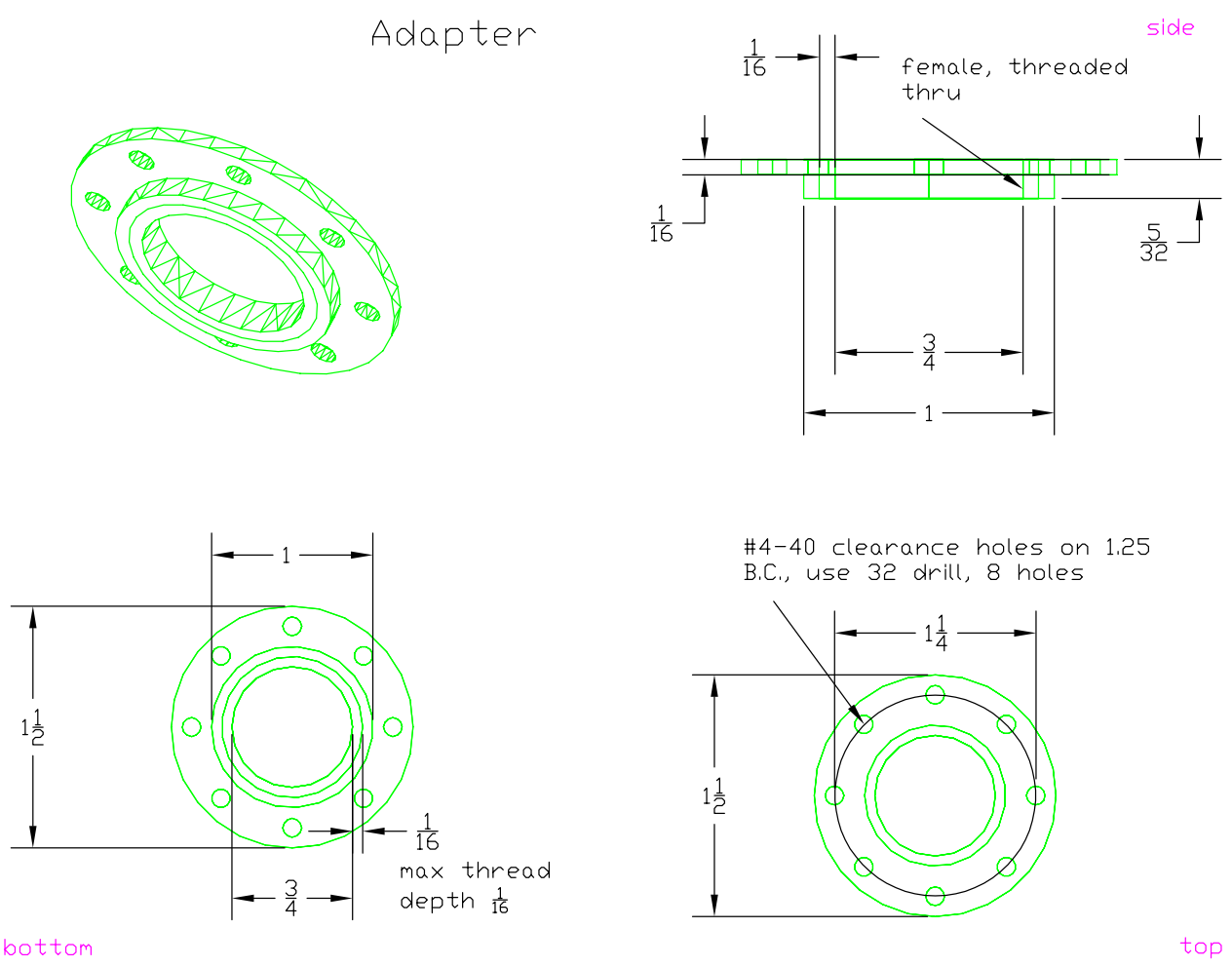

Figure A.6: Schematic of the adapter plate. The adapter is used to mount the conversion cell on the variable temperature plate inside the cryostat and provide good thermal anchoring. 


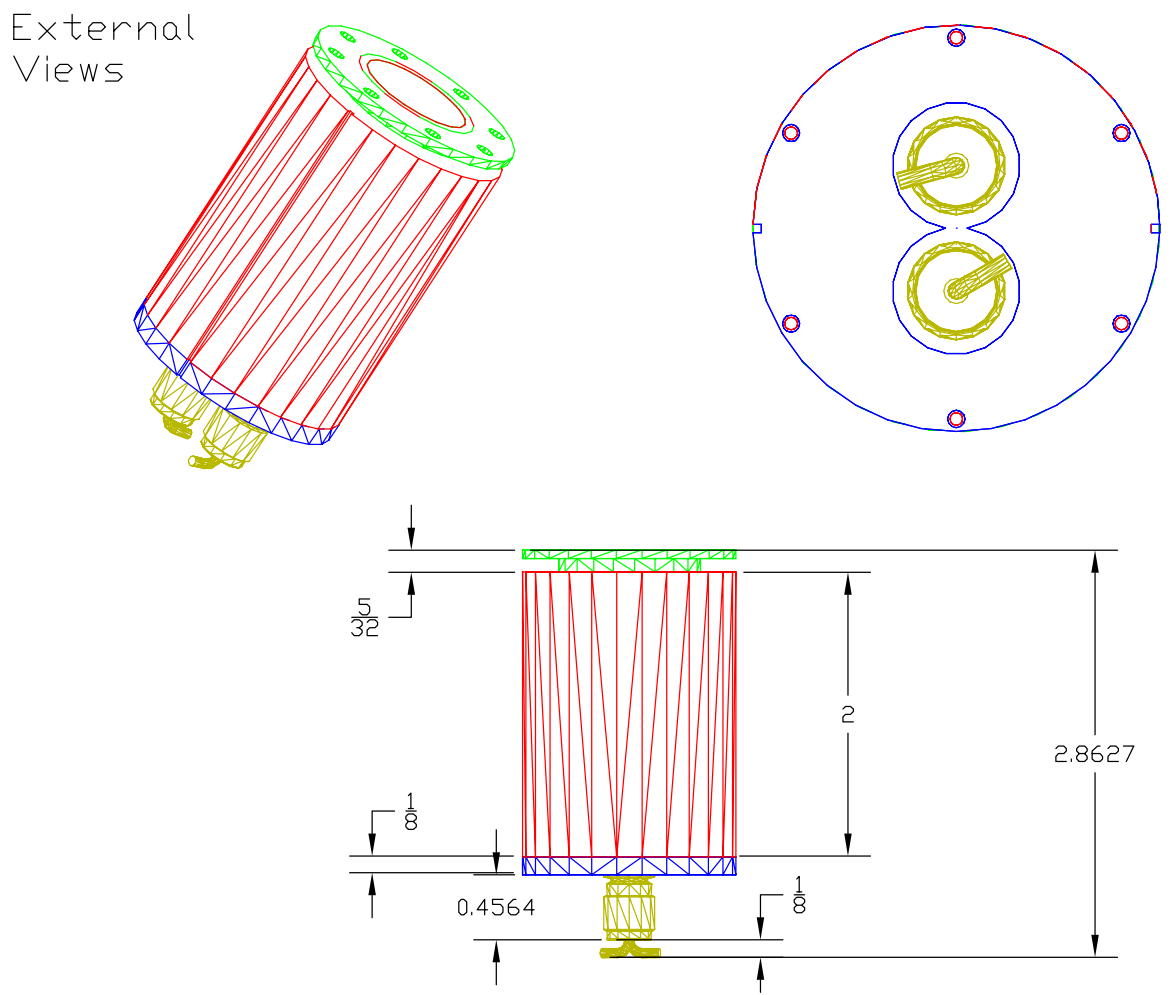

Figure A.7: Schematic of the assembled conversion cell. This design uses $90^{\circ}$ angled Swagelok adapters, which turned out to be too large for such close spacing. Straight adapters were used instead and soldered to prevent leaking. 


\section{1}

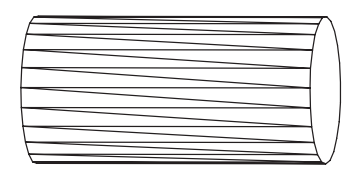

approx 4", polish sides and face end

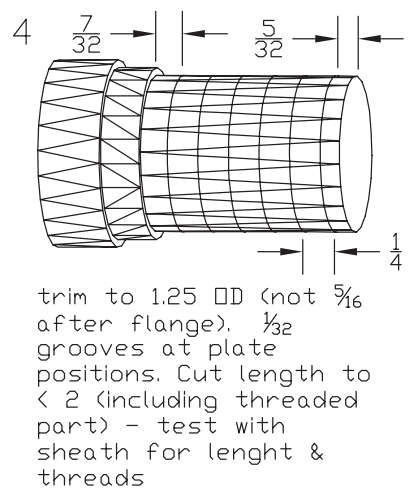

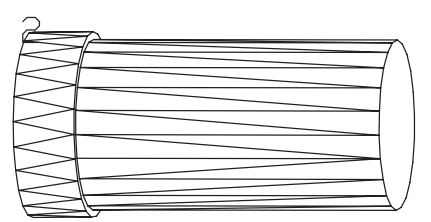

$13 / 8$ aD, trim to $<1.3666$

length of 2.5. Notch base

$1 / 16$ wide and $1 / 32$ deep

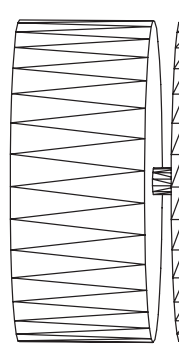

part (be careful!), $1 / 8$
flange
3

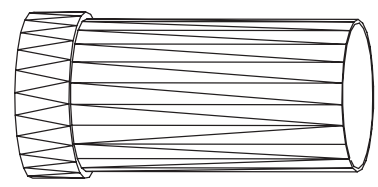

32 thr/inch, 0.02706 deep entire length, test with sheath

Figure A.8: Schematic of the steps needed to machine the baffled insert using a lathe. The steps for adding the left handed thread were not planned at this stage and were added during production. 


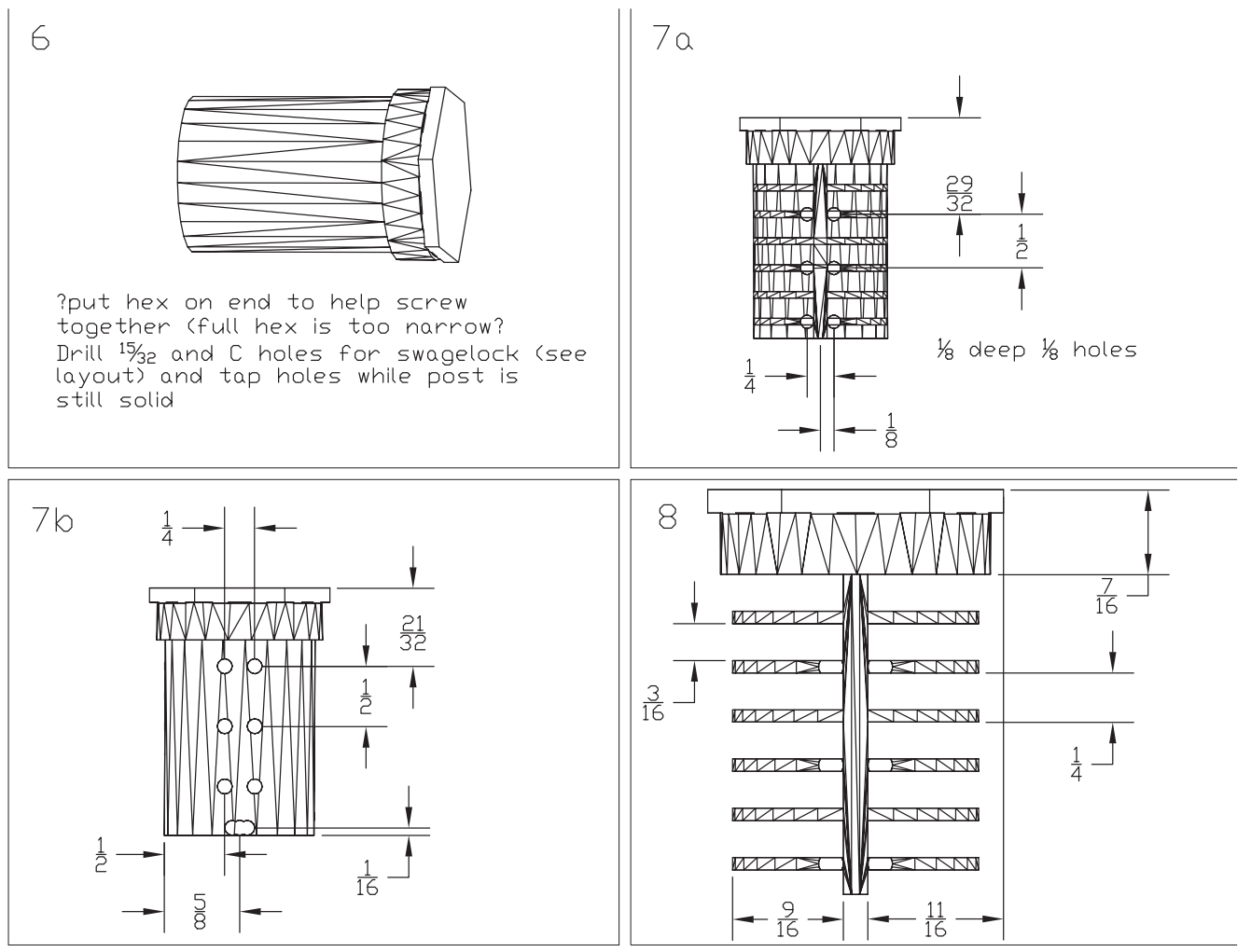

Figure A.9: Schematic of the steps needed to complete machining of the insert; this primarily uses a milling machine and a slot cutter. 

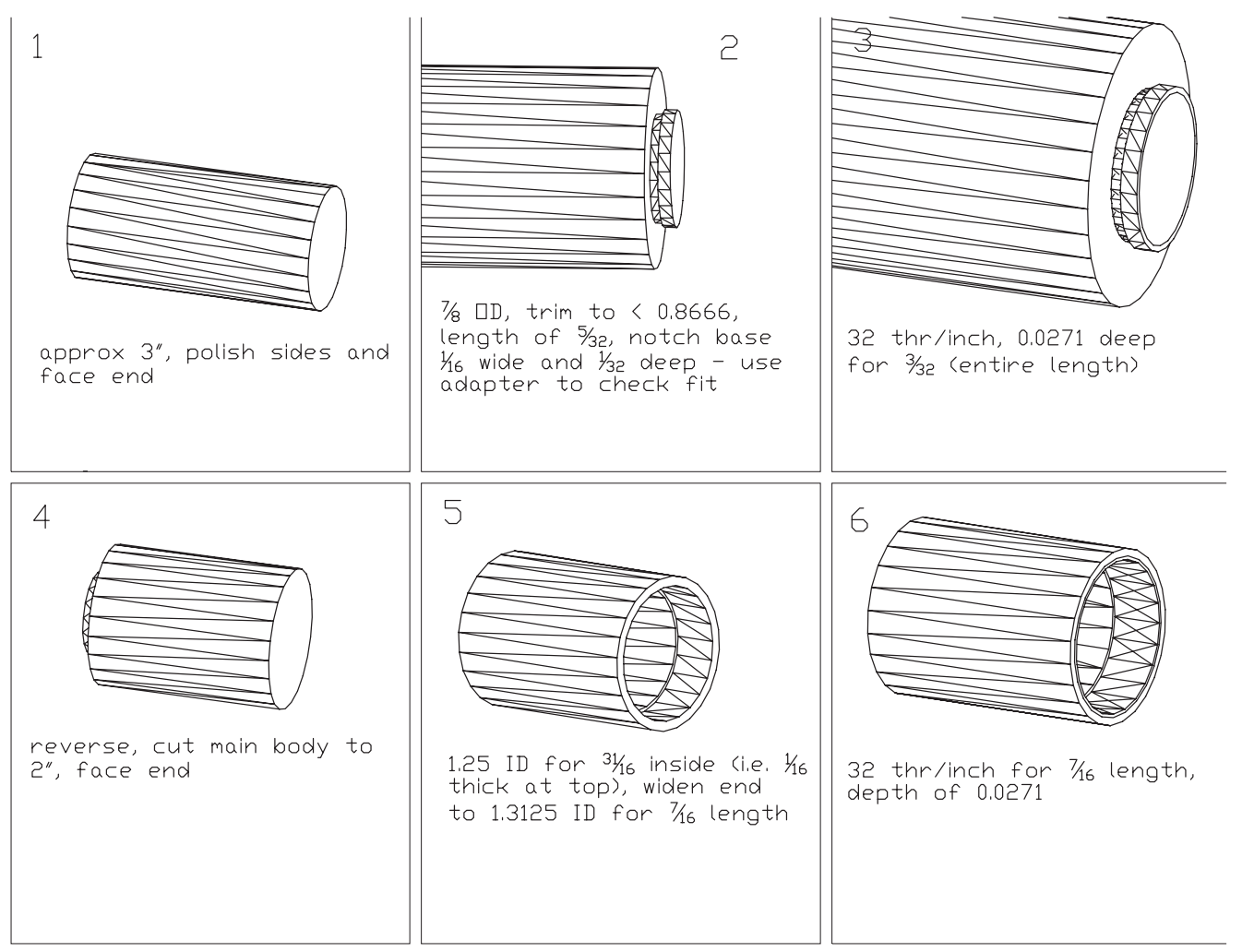

Figure A.10: Schematic of the steps needed to machine the sheath on the lathe. The steps for adding the left handed thread were not planned at this stage and were added during production. 


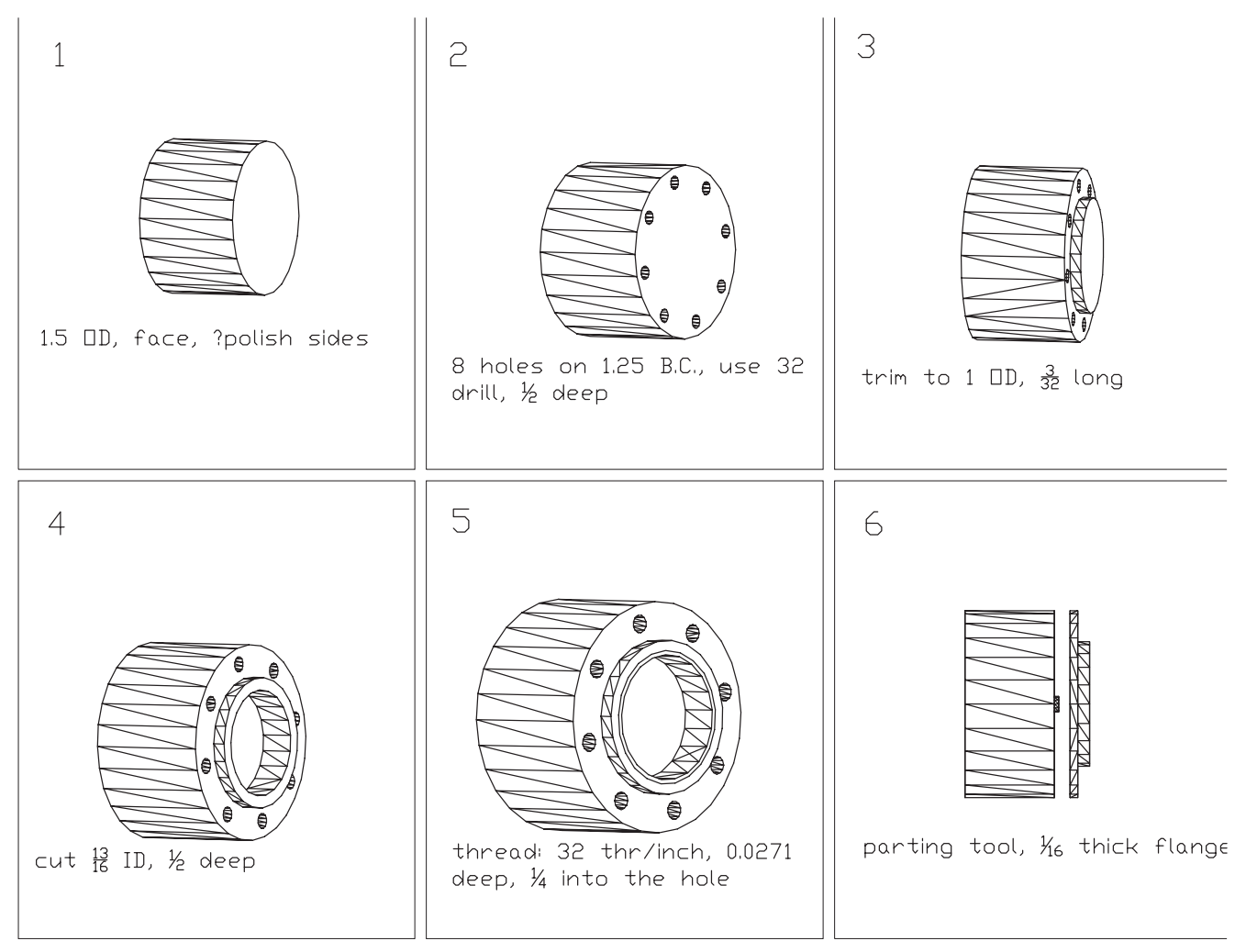

Figure A.11: Schematic of the steps needed to machine the adapter plate. 
$p-\mathrm{H}_{2}$ conversion cell, baffle design, $\approx 20 \mathrm{~K}$

A

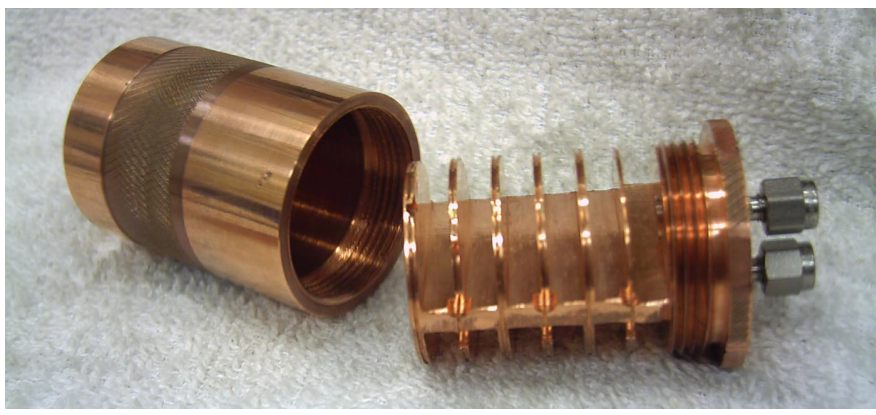

B
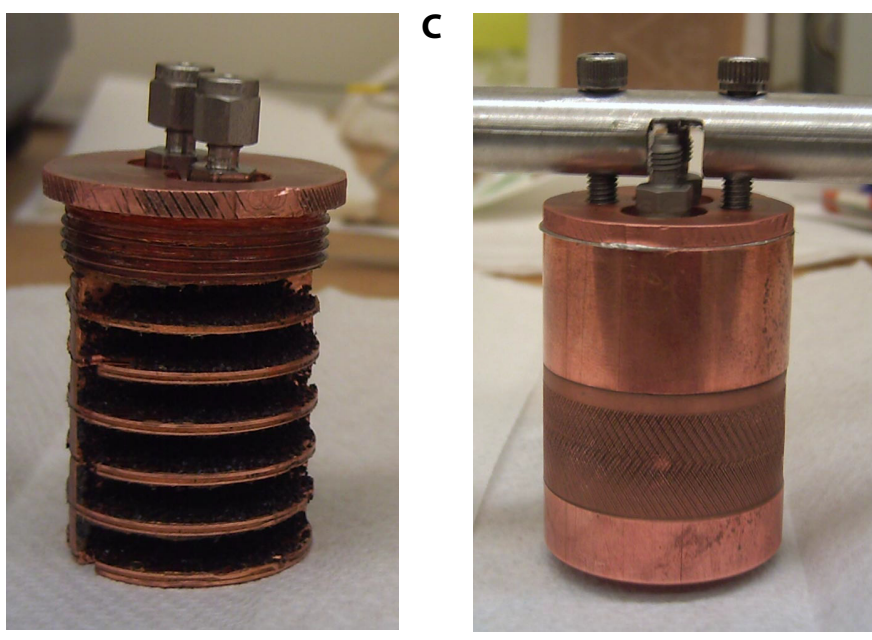

Figure A.12: Completed conversion cell. (A) The insert and sheath. (B) The insert coated with cryogrease and then coated with the ortho/para catalyst. (C) Torque wrench assembly for crushing the indium wire. 


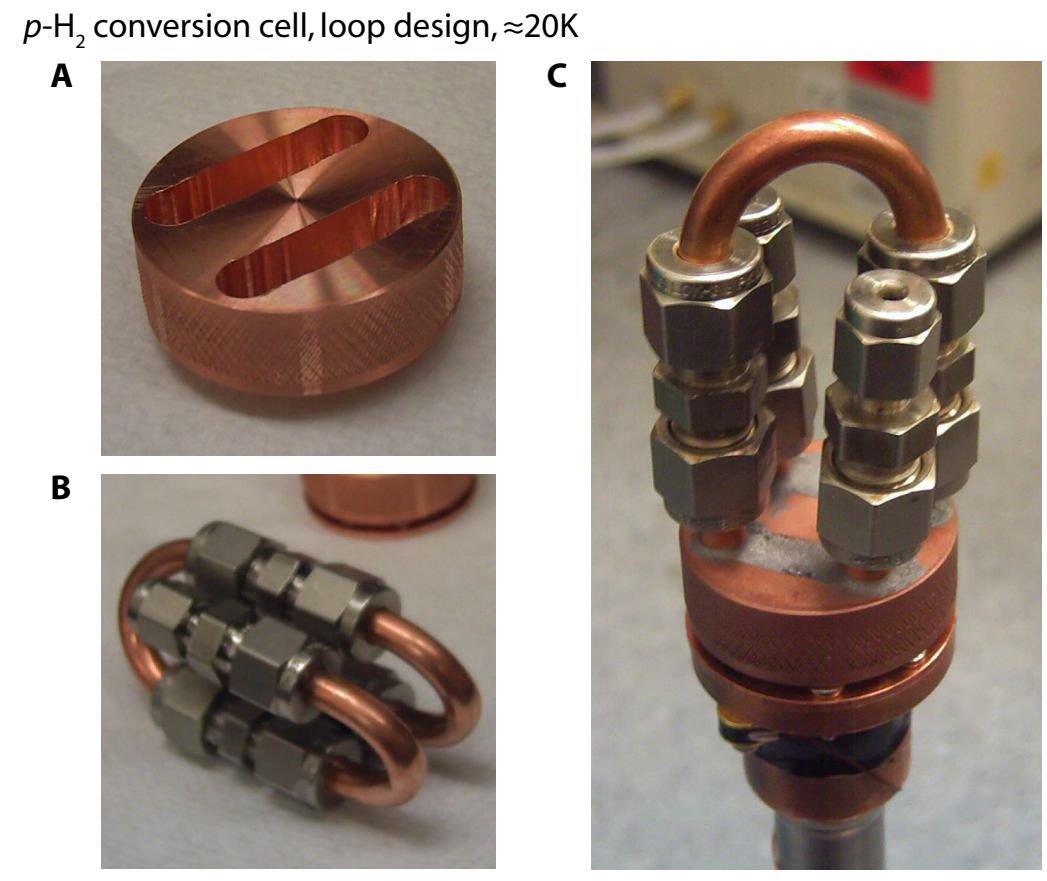

Figure A.13: This design provided a simple way to pack lots of catalyst in the tubing and provide good thermal anchoring to the mounting plate. In the end, the gas was not sufficiently cooled enough before entering the cell, so the conversion was highly dependent on flow rate 
the catalyst. Although some conversion was seen at very low flow rates, this design was not adequate.

The final design was similar to the end of the insert in fig. A.1. 3/16" Cu tubing was coiled tightly around a form with a hole for the central stem. The purpose of the form is to allow a small radius of curvature without kinking the $\mathrm{Cu}$ tubing, particularly the bend near the central stem. The purpose of this design is to provide a heat exchange coil to pre-cool the gas, analogous to the liquid $\mathrm{N}_{2}$ setup and have the central stem filled with the ortho/para catalyst for conversion. Cu braid was used for thermal anchoring. As before, the Swagelok connectors prevented problems with leaking (as opposed to NPT connections). This design works, but is rather sensitive to the flow rate of the gas. I believe this is due to the small inner diameter (ID) of the tube and the short path length through the catalyst bed. Compared to the $\mathrm{N}_{2}$ setup, the ID of the tube is almost half and the path length is half. Additionally the heat exchange coil is much shorter, without the pre-cooling coil. Although the coil is anchored to the cold finger at numerous points, this does not provide as efficient heat transfer as immersing the entire coil in the temperature bath. An improved design for the future should have more loops around the first stage, with better anchoring for improved pre-cooling, as well as using a larger diameter tube inside the coil to slow the gas velocity through the catalyst bed.

Figure A.15 shows how the first and last conversion cell designs were mounted on the cold finger. 


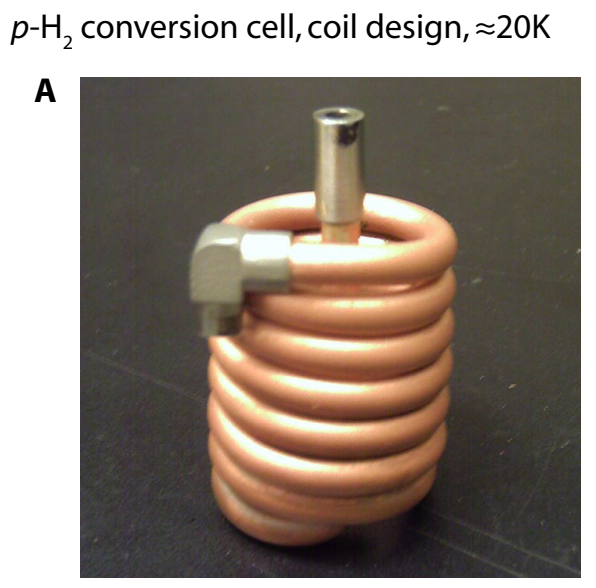

B

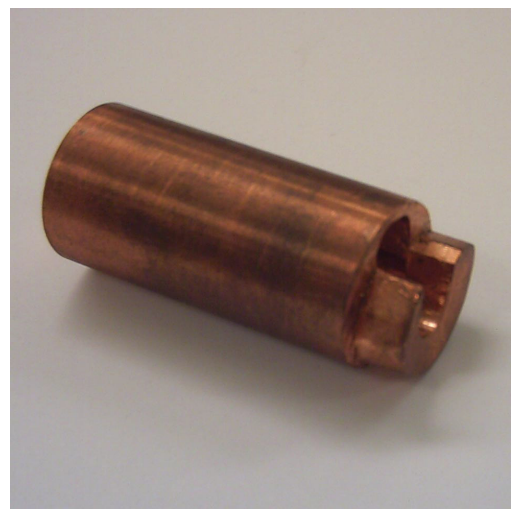

Figure A.14: A tight coil, (A), was made by wrapping 3/16" Cu tubing around a form, (B), to prevent kinks in the tubing with such small radii of curvature. The catalyst is placed in the central stem inside the coil. Thermal anchoring is provided by $\mathrm{Cu}$ braid ties to the mounting plate.

Mounting the $p-\mathrm{H}_{2}$ conversion cell
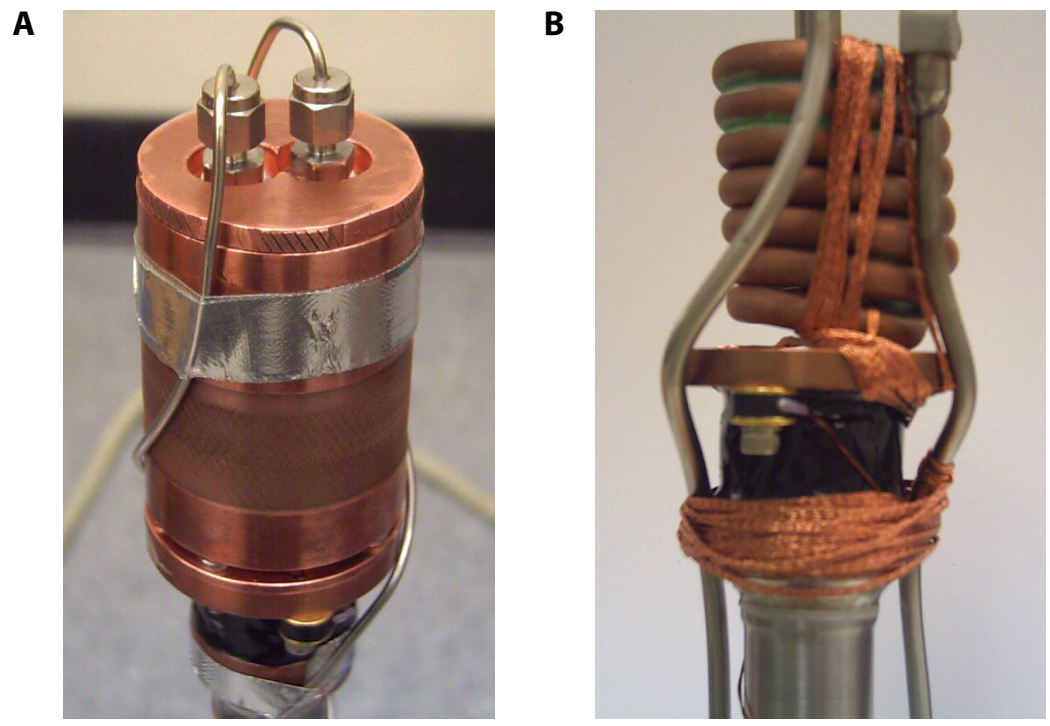

Figure A.15: Mounting and thermal anchoring of the catalyst cells to the cold finger. (A) Original baffle design. (B) Heat exchange coil design. 


\section{A.1.3 Testing the Conversion of Ortho- to Parahydrogen}

Parahydrogen is not directly observable, due to it having a total spin $I=0$ (sec. 1.3); therefore, an indirect method is needed to calibrate the fraction of $p-\mathrm{H}_{2}$ produced by the conversion cell. This is typically done by hydrogenating a reference sample in the magnet and comparing the signal from the $p-\mathrm{H}_{2}$ enhanced gas to the $n-\mathrm{H}_{2}$ gas. However, in the initial stages of building the setup, we did not have consistent catalytic activity and this method was generally too costly in terms of time and materials. Another way to measure the conversion is to monitor the amount of $o-\mathrm{H}_{2}$ remaining, as $o-\mathrm{H}_{2}$ is NMR active (i.e. $I=1)$.

The $o-\mathrm{H}_{2}$ signal is typically quite broad and can be difficult to observe, particularly due to long recycle delays. Igor Koptyug developed a simple method to observe this signal. If $\mathrm{H}_{2}$ gas is placed in or flowed through an NMR tube containing alumina pellets (approx. $1-2 \mathrm{~mm}$ diameter), a relatively sharp signal is observed for the $o-\mathrm{H}_{2}$ adsorbed on the surface of the alumina. Not only is the sensitivity improved by narrowing the $o-\mathrm{H}_{2}$ line, but also by decreasing $T_{1}$ for faster signal averaging. Note that this signal can be easily swamped by the broad signal from adsorbed water on the alumina. Thus, the alumina is dried for several hours under vacuum at $200^{\circ} \mathrm{C}$ to remove as much water as possible. Furthermore, spin echo detection is employed with an echo time of $\approx 1 \mathrm{~ms}$ to remove any residual signal from the water. Typically, signal averaging is employed in order to phase cycle the signal and further suppress spurious signals. Typical spectra are shown in fig. A.16. The pulse sequences I used can be found in sec. B.1.1.

I designed the flow setup (sec. A.2) to allow easy switching between the $p-\mathrm{H}_{2}$ 


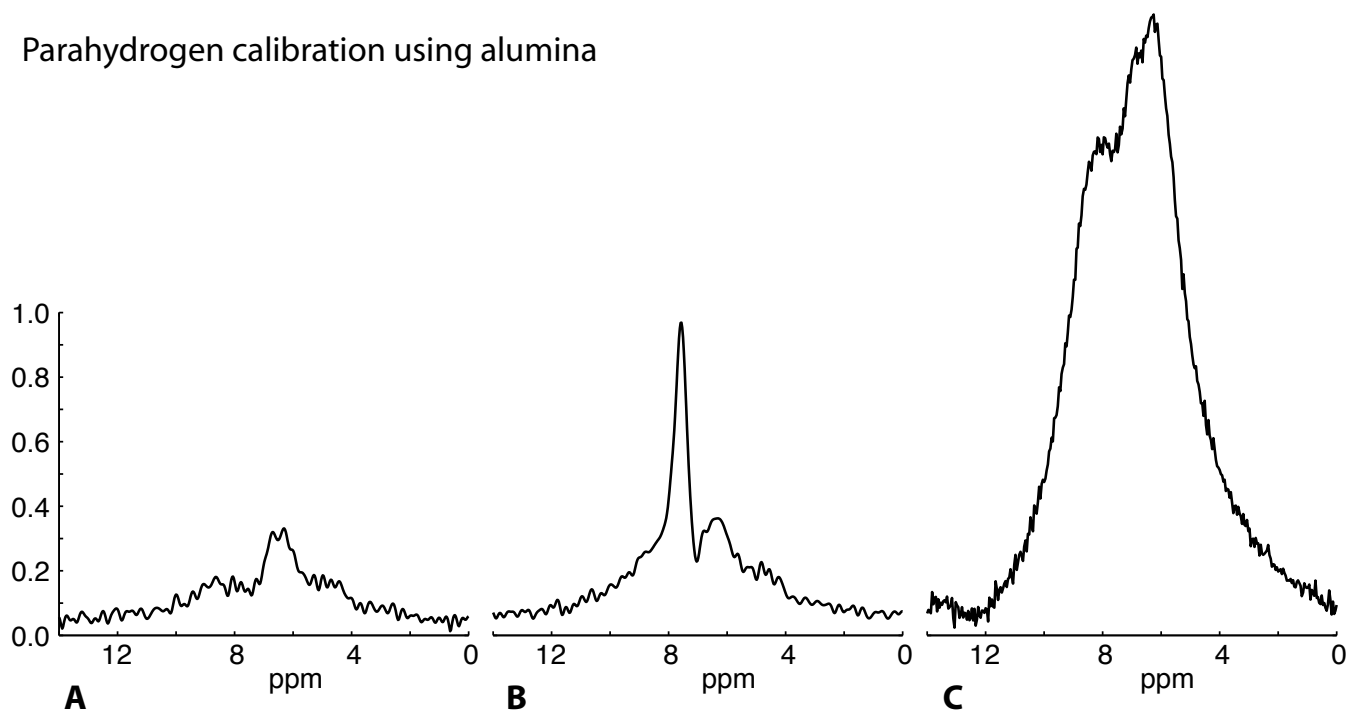

Figure A.16: $o-\mathrm{H}_{2}$ spectra obtained using Alumina. (A) Alumina under $\mathrm{N}_{2}$, (B) Alumina under $n-\mathrm{H}_{2}$, the sharp peak is from the $75 \% o-\mathrm{H}_{2}$, (C) Insufficiently dry alumina under $n-\mathrm{H}_{2}$, the $\mathrm{H}_{2} \mathrm{O}$ signal overwhelms everything else.

enhanced gas and $n-\mathrm{H}_{2}$. Using $n-\mathrm{H}_{2}$ provides a reference signal for calculating the actual fraction of $p-\mathrm{H}_{2}$ coming from the converter. Given that $n-\mathrm{H}_{2}$ is $75 \% o-\mathrm{H}_{2}$, the fraction of $p-\mathrm{H}_{2}$ in the $\mathrm{H}_{2}$ flow from the converter is

$$
f=1-\left(\frac{3}{4} r\right)
$$

where $r$ is the ratio of the two signals (from the conversion cell and from the main cylinder) and $f$ is the fraction of $p-\mathrm{H}_{2}$ in the ortho/para $\mathrm{H}_{2}$ mixture.

This method is used to track the fraction of $p-\mathrm{H}_{2}$ versus the flow rate through the converter. An example plot is given in fig. A.17. This shows that the liquid $\mathrm{N}_{2}$ converter is quite robust over a large range of flow rates.

Some common problems with this method should be noted. First, changing the flow rate changes the amount of $\mathrm{H}_{2}$ in the sample cell. This will impact the calibration 


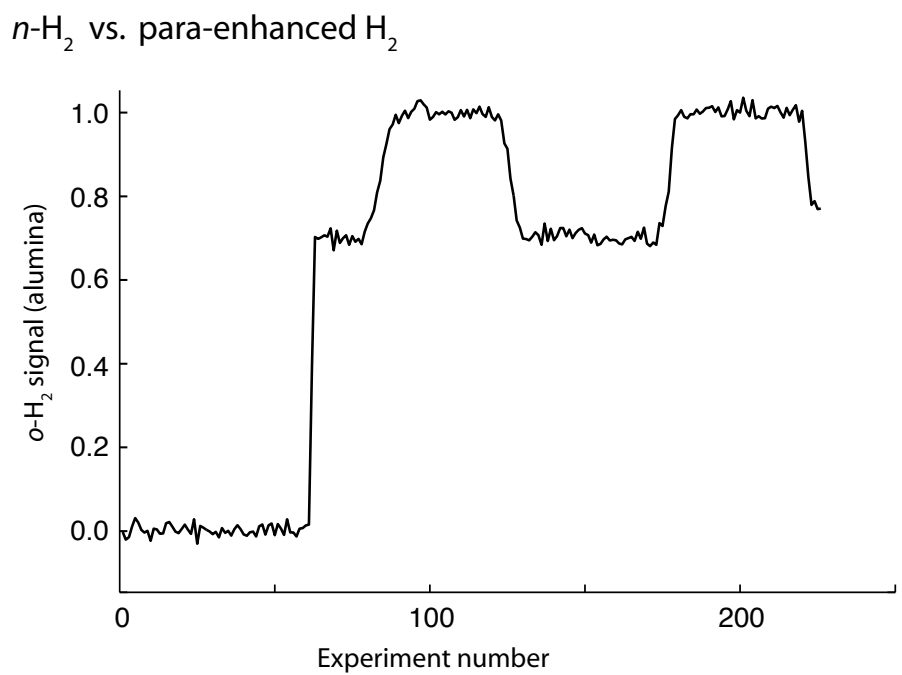

Figure A.17: Calibrating the fraction of $p-\mathrm{H}_{2}$. The background signal is collected with $\mathrm{N}_{2}$ flowing through the cell. Signal is collected for both $n-\mathrm{H}_{2}\left(25 \% p-\mathrm{H}_{2}\right)$ and para-enriched $\mathrm{H}_{2}$. The ratio of the signal strengths gives the fraction of $p-\mathrm{H}_{2}$, according to eq A.2.

of $p-\mathrm{H}_{2}$ fraction vs. flow rate, but is easily taken into account by acquiring a reference spectrum of $n-\mathrm{H}_{2}$ at each flow rate. More importantly, in the limit of pure $p-\mathrm{H}_{2}$, it is difficult to tell the difference between an absence of $o-\mathrm{H}_{2}$ and an absence of any $\mathrm{H}_{2}$. This is a real problem with the cryostat when the $\mathrm{H}_{2}$ liquifies or freezes in the tubing, blocking the flow. The easiest way to address this is to ensure that gas is flowing, e.g. with a flow meter at the outlet of the setup, as well as by hydrogenating a standard sample and observing the resulting PASADENA signal. Finally, complete conversion is not possible for faster flow rates, as shown in table A.1. When the flow rate is too fast, the gas does not have enough time to cool down completely and the time spent in the catalyst bed is shorter, reducing the probability of conversion. 


\begin{tabular}{cc}
$\mathrm{H}_{2}$ flow rate $/ \mathrm{sccm}$ & $\% p-\mathrm{H}_{2}$ \\
\hline 80 & $48 \%$ \\
140 & $48 \%$ \\
200 & $42 \%$ \\
260 & $35 \%$
\end{tabular}

Table A.1: The resulting fraction of $p-\mathrm{H}_{2}$ from the liquid $\mathrm{N}_{2}$ conversion cell at several flow rates. Faster flow rates result in incomplete conversion.

\section{A.2 Gas Flow Setup}

After joining Alex's lab, my first real task was to design and build a simple flow setup for producing a constant flow of $p-\mathrm{H}_{2}$ enhanced gas for delivery to a sample cell inside the bore of the magnet. This seemingly simple task has evolved into a constant and ever changing project over the past three and half years; I have continued to modify, improve and sometimes drastically change the entire setup. The core features have remained the same, however. Every iteration of the setup was designed with the following requirements:

- A constant flow of $\mathrm{H}_{2}$ gas, that can easily be switched between $p-\mathrm{H}_{2}$ enriched or $n-\mathrm{H}_{2}$, is delivered to a sample cell inside the bore of the magnet.

- The setup must have built-in safety controls to minimize the hazards of working with $\mathrm{H}_{2}$ (and other flammable gases).

- Provide both manual and automated control of the gas flow.

The specific details of the setup, including both design and proper use, are given below. 


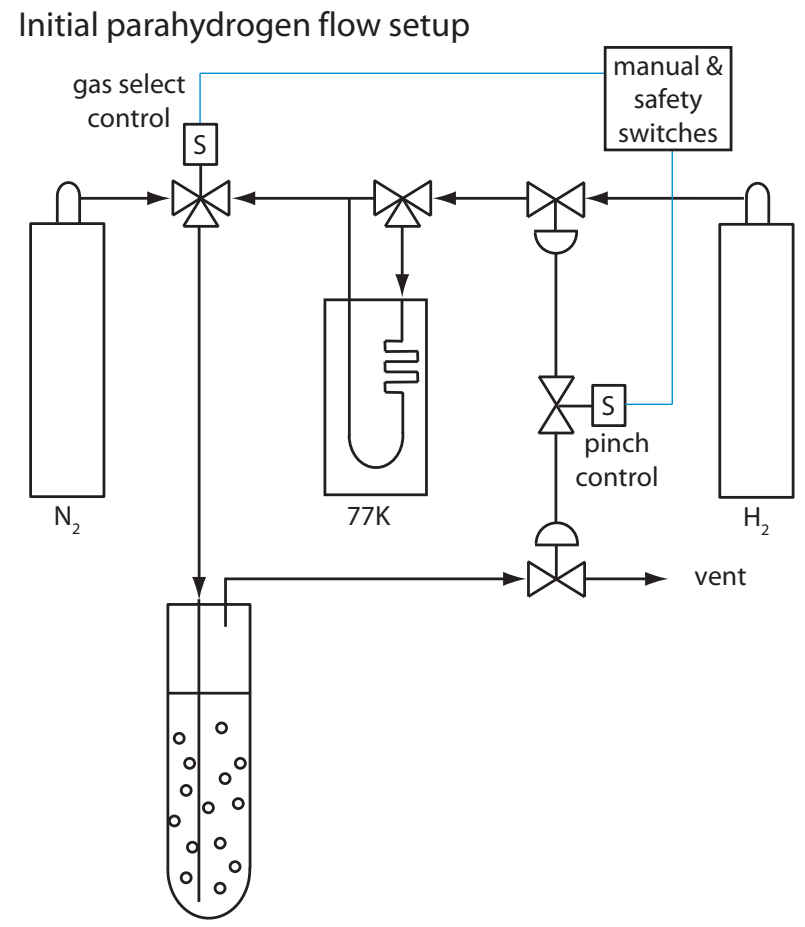

Figure A.18: The initial flow design used a single pneumatically actuated valve for both the safety $\mathrm{H}_{2}$ shutoff and as the pinch for closing the inlet and the outlet of reaction cell to stop bubbling. Another second valve selected either $\mathrm{H}_{2}$ or $\mathrm{N}_{2}$ gas to enter the reaction cell.

\section{A.2.1 Gas Manifold}

The most important feature of the gas manifold is enriching the fraction of $p-\mathrm{H}_{2}$ (sec. A.1) and delivering this $p-\mathrm{H}_{2}$ enhanced gas to the magnet. The initial design of the flow setup met this goal with a simple manifold containing one switch to select the input gas $\left(\mathrm{H}_{2}\right.$ or $\left.\mathrm{N}_{2}\right)$ and another switch that simultaneously closed two valves, the input and the exhaust, as depicted in fig. A.18. The valves were controlled by manual switches as well as safety monitors (sec. A.2.3).

This flow setup worked quite well for calibrating the conversion of $\mathrm{H}_{2}$ gas to $p-\mathrm{H}_{2}$ and for simple bubbling experiments. Typically, the solution would be bubbled with $\mathrm{N}_{2}$ 


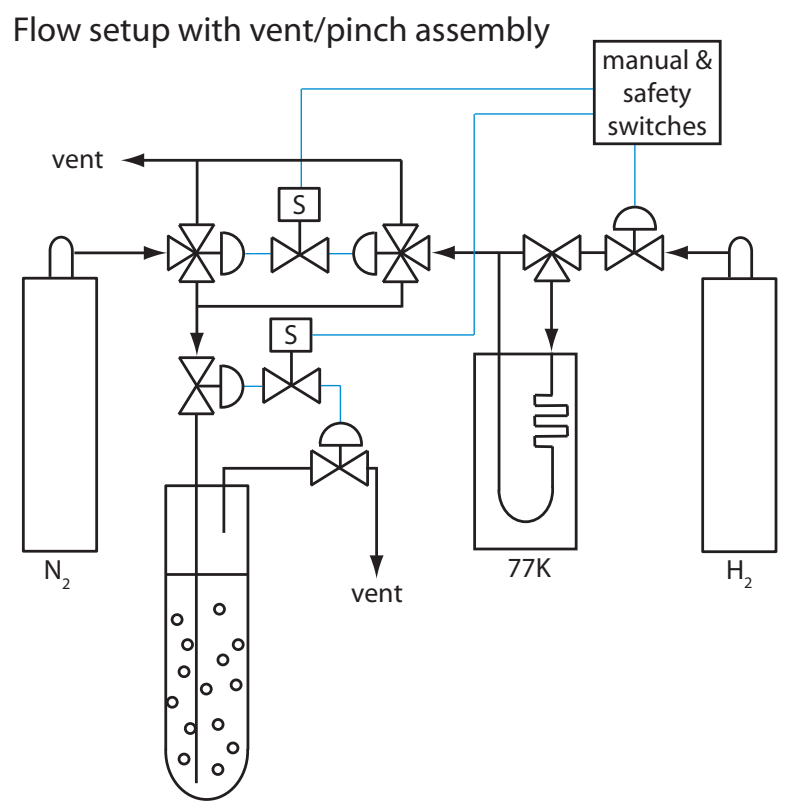

Figure A.19: The second flow design moved the pinch valve closer to the magnet to reduce the volume of gas that is pinched. Furthermore the $p-\mathrm{H}_{2}$ converter was moved outside the pinch volume to reduce continued bubbling after pinching. A new $\mathrm{H}_{2}$ safety valve was added. A more sophisticated vent setup ensured that gas was always flowing to prevent pressure buildups while switching between $\mathrm{N}_{2}$ and $\mathrm{H}_{2}$.

first, then the input gas would be switched to $p-\mathrm{H}_{2}$ enriched $\mathrm{H}_{2}$. After bubbling for a few seconds, the pinch valve was closed and the spectrum acquired. The main problem with this setup is that a pressure differential builds up behind the conversion cell in the liquid $\mathrm{N}_{2}$ dewar, so even after the pinch valve is closed, gas continues to bubble inside the sample. This causes problems when a short dead time was desired, i.e., when it is important to acquire a spectrum immediately after bubbling, without further hydrogenation taking place.

The second flow setup moved the $p-\mathrm{H}_{2}$ converter outside of the pinch valve as well as moving the switches closer to the magnet to reduce the path length enclosed by the pinch (fig. A.19). All of this reduces the dead time and allows better control over the bubbling 
behavior of the gas. The gas selection switch was changed to always vent the gas that is not directed into the reaction cell; this prevents pressure buildups that lead to excessively violent bubbling. A picture of the vent and pinch assembly is given in fig. A.21D. The setup was also modified to allow the pulse program to control the switches (sec. A.2.2) via TTL lines.

The final change to the flow setup was a complete rebuilding of the manifold to place it on a workbench. The previous design was kept, but three things were added: a cryostat for producing larger fractions of $p-\mathrm{H}_{2}$ (sec. A.1.2), a manifold for filling small cylinders with a gas mixture (typically $\mathrm{N}_{2}, p-\mathrm{H}_{2}, o-\mathrm{H}_{2}$ and an alkene) and a freeze/pump/thaw setup. The final flow layout and pictures of the setup are given in figs. A.20 and A.21. Note that the freeze/pump/thaw and cylinder filling stations are each designed so the sample or cylinder can be purged of $\mathrm{O}_{2}$. Each station has a $\mathrm{N}_{2}$ line, a vent line and a vacuum line. The typical application is to fill the sample or cylinder with high pressure $\mathrm{N}_{2}$, vent this to atmospheric pressure, and then pump on it. This is repeated several times to remove any possible $\mathrm{O}_{2}$ contamination. After purging the $\mathrm{O}_{2}, p-\mathrm{H}_{2}$ enhanced $\mathrm{H}_{2}$ is added. In the case of the cylinder filling station, additional gases can also be added, e.g. propylene (ch. 3 and $4)$.

\section{A.2.2 Flow Control}

I designed the flow setup to have three types of control over the gas flow: automatic flow control via safety monitoring devices, manual flow control via switches, and spectrometer control via TTL lines. These three types of control change the state of three 


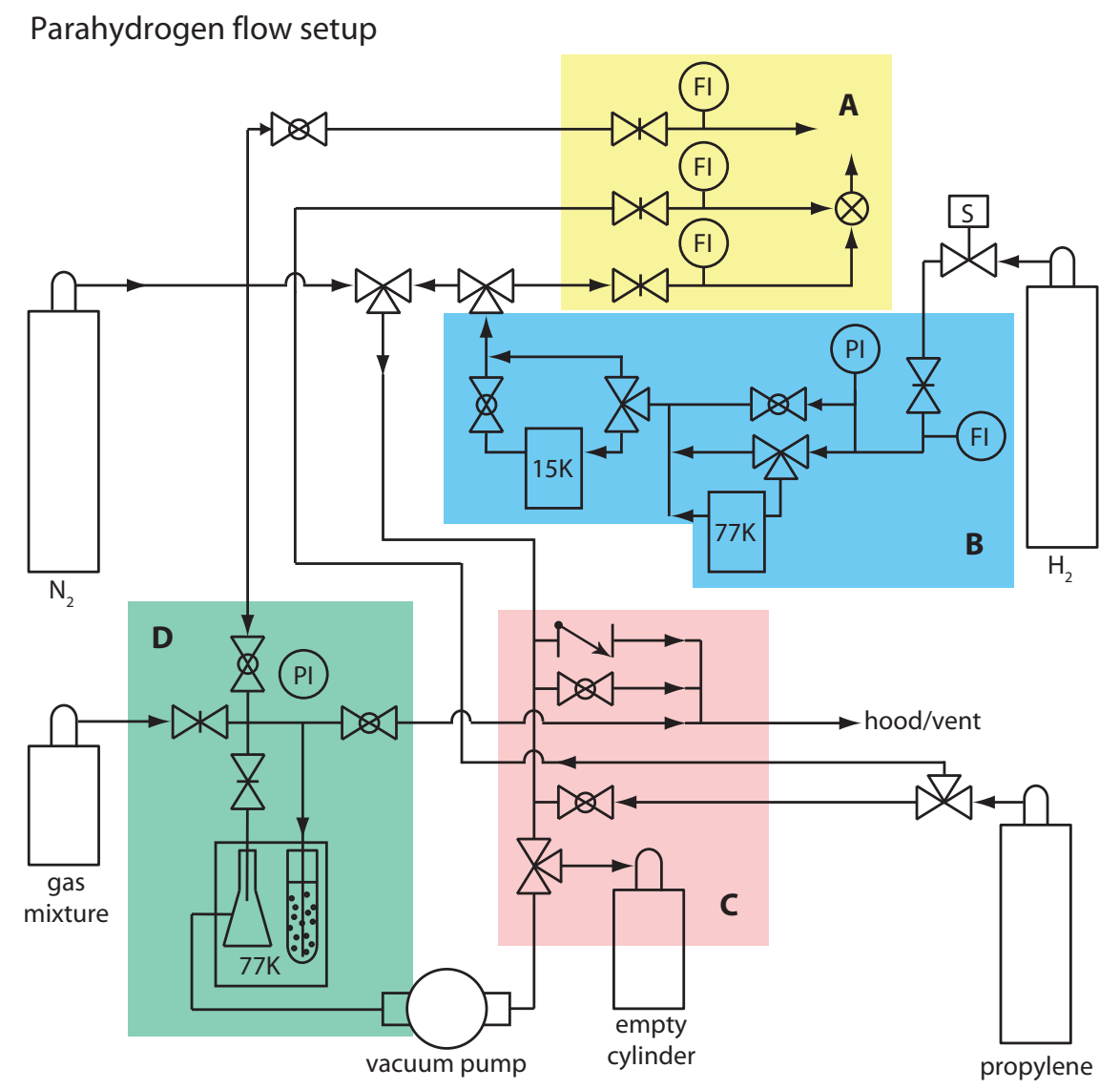

Figure A.20: The current flow design. (A) The same pinch/vent design described in fig. A.19 is used to deliver gas directly into the reaction cell in the magnet bore. An improved mass flow control panel and the ability to mix additional gases with the $\mathrm{H}_{2}$ stream are added. (B) The liquid $\mathrm{N}_{2}$ stage for producing $p-\mathrm{H}_{2}$ is the same as the previous designs. A compressed ${ }^{3} \mathrm{He}$ cryostat is added for producing flows of up to $98 \% p-\mathrm{H}_{2}$. (C) A manifold for filling small cylinders with $p-\mathrm{H}_{2}$ and other gases is provided. (D) A freeze/pump/thaw manifold is provided for ALTADENA experiments. 


\section{Parahydrogen flow setup}
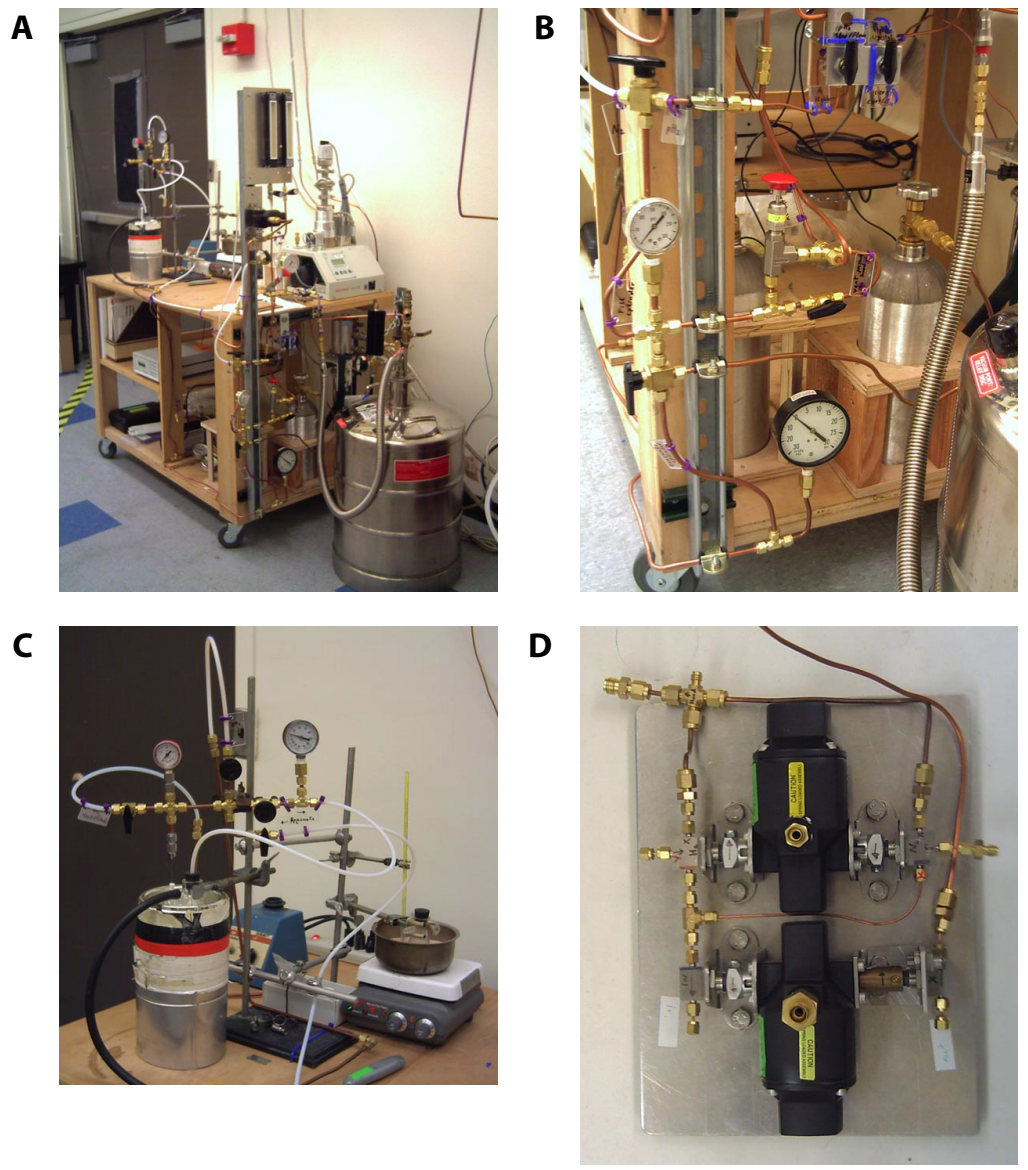

Figure A.21: Images of the flow setup. (A) Main $p-\mathrm{H}_{2}$ workbench. (B) Cylinder filling station. (C) Freeze/pump/thaw setup. (D) Vent/pinch assembly that controls the gas flow. 
switches: $\mathrm{H}_{2}$ source, open/closed; $\mathrm{H}_{2}$ gas directed to the vent or the reaction cell; and $\mathrm{N}_{2}$ gas directed to the vent of the reaction cell. These three switches are all pneumatically actuated, driven by solenoid valves on the control panel, as shown in fig. A.22.

The automated safety control is based on a self-latching relay; a push-button latches the relay and the circuit has power unless one of the safety monitors falls outside the normally operating range (e.g. flow rate in the mini-hood is too low or $\mathrm{H}_{2}$ concentration is too high). The purpose of the latching circuit is to ensure that once the panel loses power due to a fault, the power remains off until manually relatched by the push-button. Furthermore, the solenoid valves are wired such that their unpowered state is the safest configuration: $\mathrm{H}_{2}$ source is closed, $\mathrm{H}_{2}$ switch is venting $\left(\mathrm{N}_{2}\right.$ is directed to the reaction cell) and the pinch is open. Even if the safety monitors return to a safe level, the circuit must be manually reset to re-latch the circuit (see discussion in sec. A.2.3).

The manual controls are two or three way switches, as shown in figs. A.23 and A.22. The $\mathrm{H}_{2}$ source is either open or closed (default closed). The vent switch has three positions: $\mathrm{H}_{2}$ to reaction cell ( $\mathrm{N}_{2}$ to vent), $\mathrm{H}_{2}$ to vent $\left(\mathrm{N}_{2}\right.$ to reaction cell), and TTL controlled (default is $\mathrm{H}_{2}$ to vent). The pinch switch also has three positions: open, closed and TTL controlled (default is open).

When the vent and/or pinch switches are placed in the TTL position, the spectrometer can trigger the two states of the control valves. I had to add two TTL lines to the spectrometer (see the Bruker Avance Service Handbook for instructions). Register 8 was unused so I designated register 8 , channel 7 for the vent and register 8 , channel 8 for the pinch. The TTL signal is triggered between high and low using 
Safety/automation setup

A

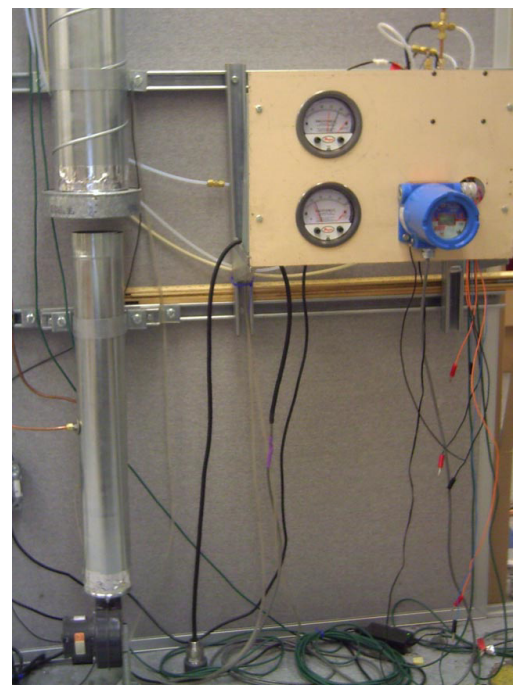

B
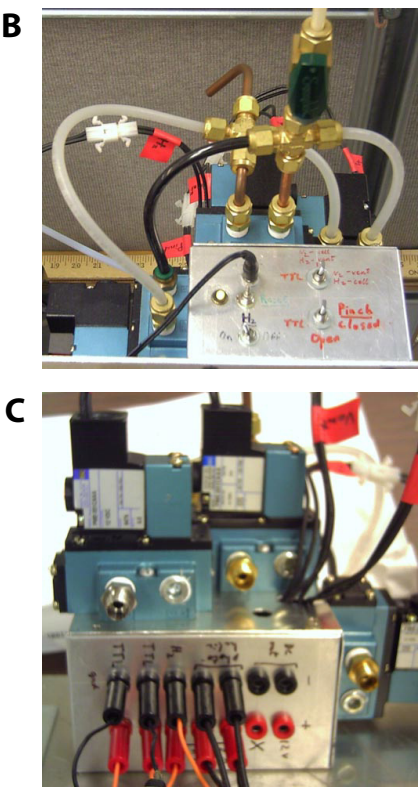

Figure A.22: Control panel and monitoring devices for automation and safety. (A) Minihood for $\mathrm{H}_{2}$ exhaust. The fan at the bottom mixes the exhaust with air to reduce the concentration below the flammability limit. Two pressure gauges and a $\mathrm{H}_{2}$ concentration meter monitor the exhaust. (B) Control panel with switches to control the three main valves: $\mathrm{H}_{2}$ flow, gas selection and pinching of the reaction cell. (C) Interface for TTL lines, monitoring devices and solenoid valves.

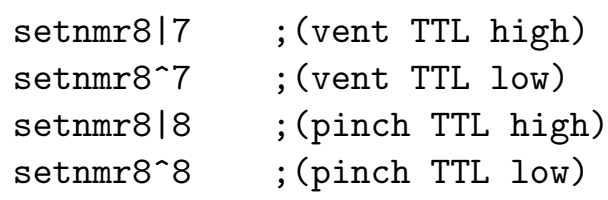

within the Bruker pulse sequence.

\section{A.2.3 Safety Controls}

The primary safety concerns when working with $\mathrm{H}_{2}$ gas are the flammability and explosion hazards. While these are concerns with any flammable gas, $\mathrm{H}_{2}$ is particularly problematic because its auto-ignition energy is lower than other common fuels; an invisible 


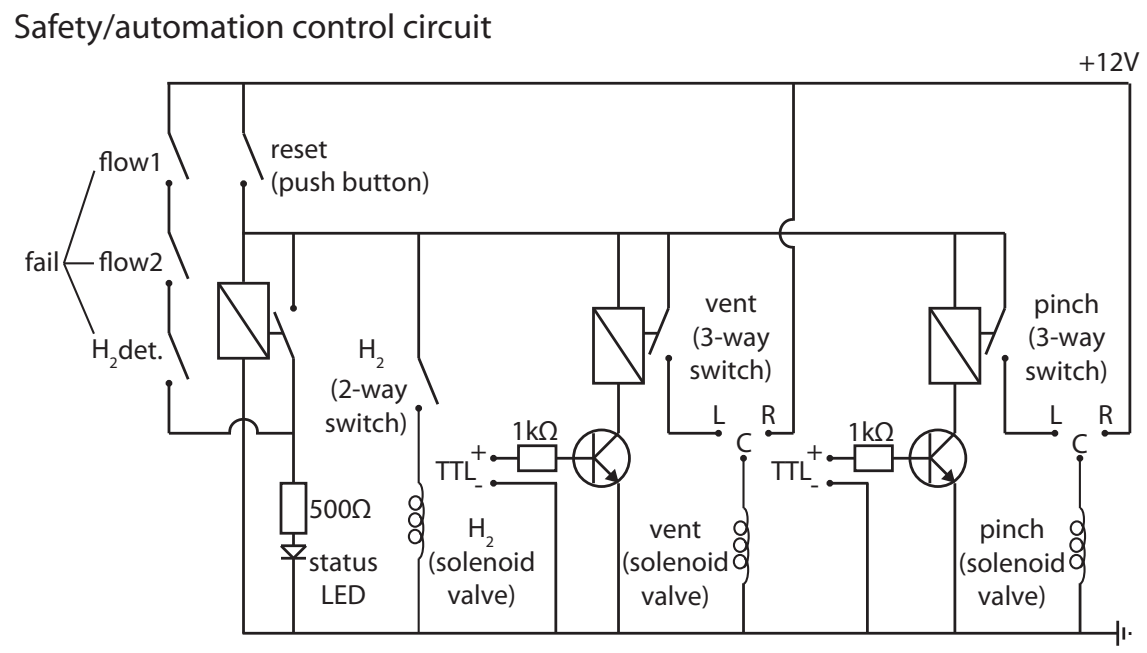

Figure A.23: Safety and Control circuit. The flow switches are driven by high pressure air which, in turn, is driven by solenoid valves. Each solenoid valve is triggered either manually, by closing the circuit, or by a TTL driven relay. The TTL circuit uses a transistor to ground the relay when the TTL signal is high, closing the circuit and turning on the solenoid valve.

spark or discharge from static electricity is enough to ignite $\mathrm{H}_{2}$ gas if its concentration is $>4 \%$. To further compound this issue, $\mathrm{H}_{2}$ has a very low electrical conductivity and builds up static charges simply from flowing through a tube. Additionally, the small size of $\mathrm{H}_{2}$ makes leaks a particular concern. Connections that are leak-tight for $\mathrm{O}_{2}$ or $\mathrm{N}_{2}$ can easily leak $\mathrm{H}_{2}$ gas. Plastic tubing is problematic, not only for the problem of building up a static charge, but also because many polymers are permeable to $\mathrm{H}_{2}$.

The flow setup was designed specifically to address these concerns. The issue of gas leaks and permeability is addressed by using $\mathrm{Cu}$ tubing and Swagelok connectors. Every connection is tested under high pressure with a flammable gas detector. The ignition hazard is abated by using $\mathrm{Cu}$ tubing and thoroughly grounding the tubing. Furthermore, the automated switches are pneumatically actuated to allow the electrical components to be further removed from the $\mathrm{H}_{2}$ manifold. Only a short section of plastic tubing is used 
to connect the reaction cell to the pinch manifold. This is kept as short as possible, but is necessary to allow the sample to be moved in and out of the magnet bore. To prevent the build up of $\mathrm{H}_{2}$ gas in the lab, each section of the flow setup has a line that vents the exhaust to a central mini-hood. $\mathrm{H}_{2}$ cannot simply be vented into the building's exhaust system, as this would present a potential explosion hazard. The final design, approved by the department EH\&S specialist, is a mini-hood designed to thoroughly mix the exhaust with air to reduce the concentration. Several safety devices monitor this process to ensure that there is a constant air flow and that the $\mathrm{H}_{2}$ concentration is below $4 \%$. If any of these monitors fall outside their specified range, the automated circuit (sec. A.2.2) shuts off the $\mathrm{H}_{2}$ flow. This circuit is designed to require a physical reset once the monitors are back in normal operating range; this prevents the $\mathrm{H}_{2}$ flow from resuming unless someone is there to turn it back on. 


\section{Appendix B}

\section{Source Code}

\section{B.1 Pulse Programs}

The following is a summary of the most important pulse sequences that I have used in my research. The implementation is included along with some notes when using these pulse sequences. The source code is either Bruker or Varian, as indicated.

\section{B.1.1 Calibrating $p-\mathrm{H}_{2}$ Fraction}

$\mathrm{T}_{2}$ filter, parahydrogen calibration

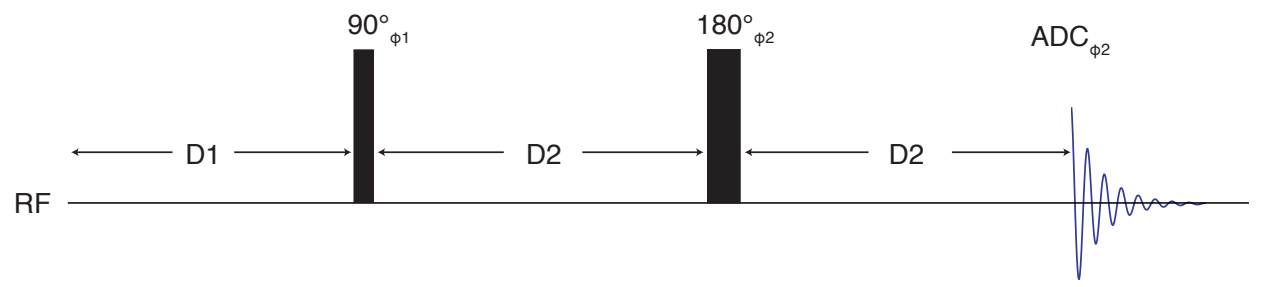

Figure B.1: A spin echo is used with a $T E$ of about $1 \mathrm{~ms}$ to allow the surface adsorbed water to relax before acquisition of the $o-\mathrm{H}_{2}$ signal. To obtain meaningful results, the $o-\mathrm{H}_{2}$ signal strength must be compared with $n-\mathrm{H}_{2}$ at the same flow rate. 
As discussed in sec. A.1.3, the fraction of $p-\mathrm{H}_{2}$ is inferred from the relative amount of $o-\mathrm{H}_{2}$ in the sample compared to $n-\mathrm{H}_{2}$. This is measured using alumina to provide a sharper resonance. However, even after drying under vacuum, the water peak can obscure the $o-\mathrm{H}_{2}$ signal. This is easily remedied using spin echo detection, with an echo time $(T E)$ long enough for the solid signal (i.e. surface bound water) to relax. A complete phase cycle is provided, but typically only 4 or 8 scans are collected.

The following pulse programs are for the Bruker Avance spectrometer using xWinNMR or TopSpin.

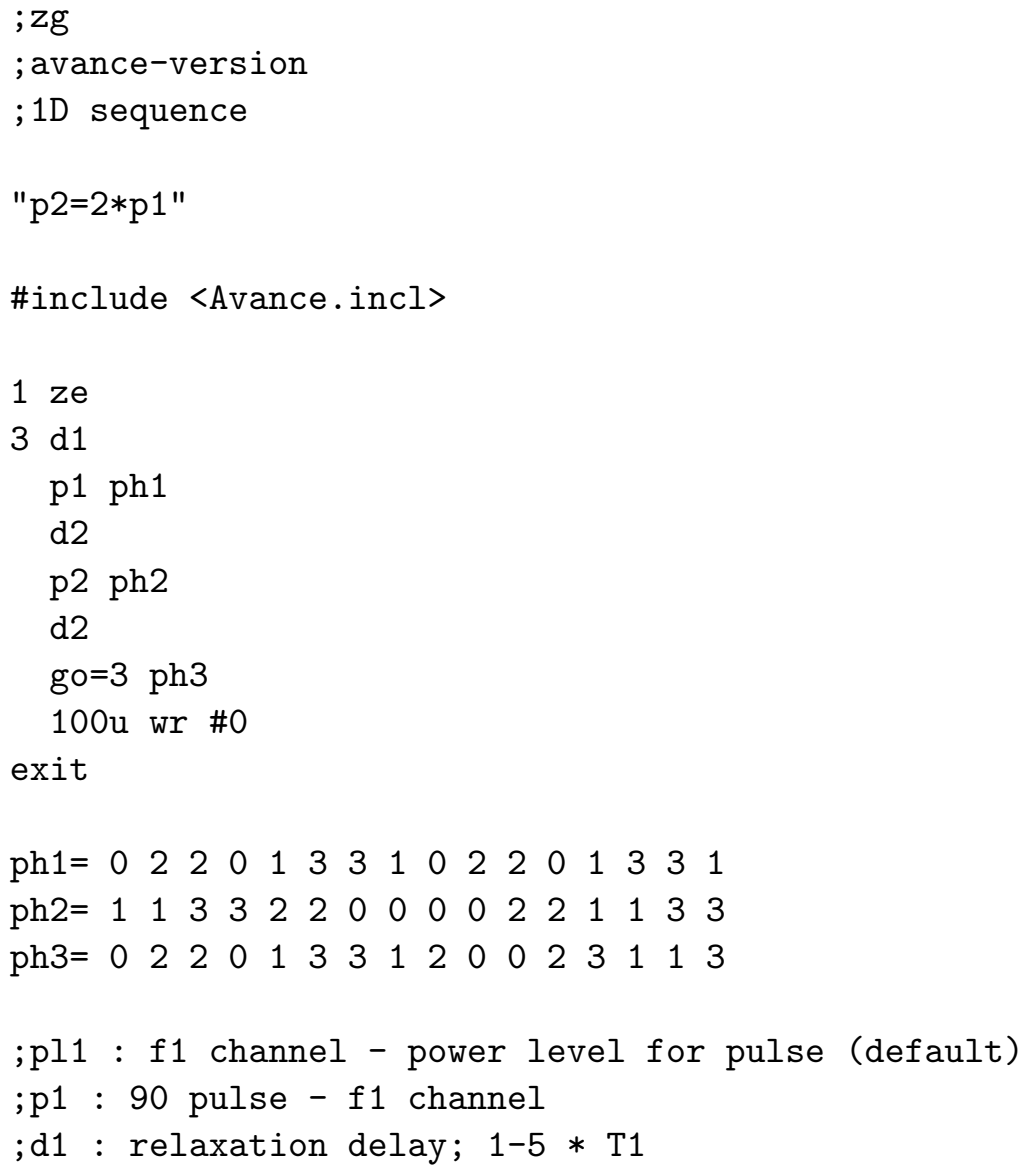

A variation of this code that uses the TTL lines to measure the $o-\mathrm{H}_{2}$ signal using 
stopped-flow acquisition is

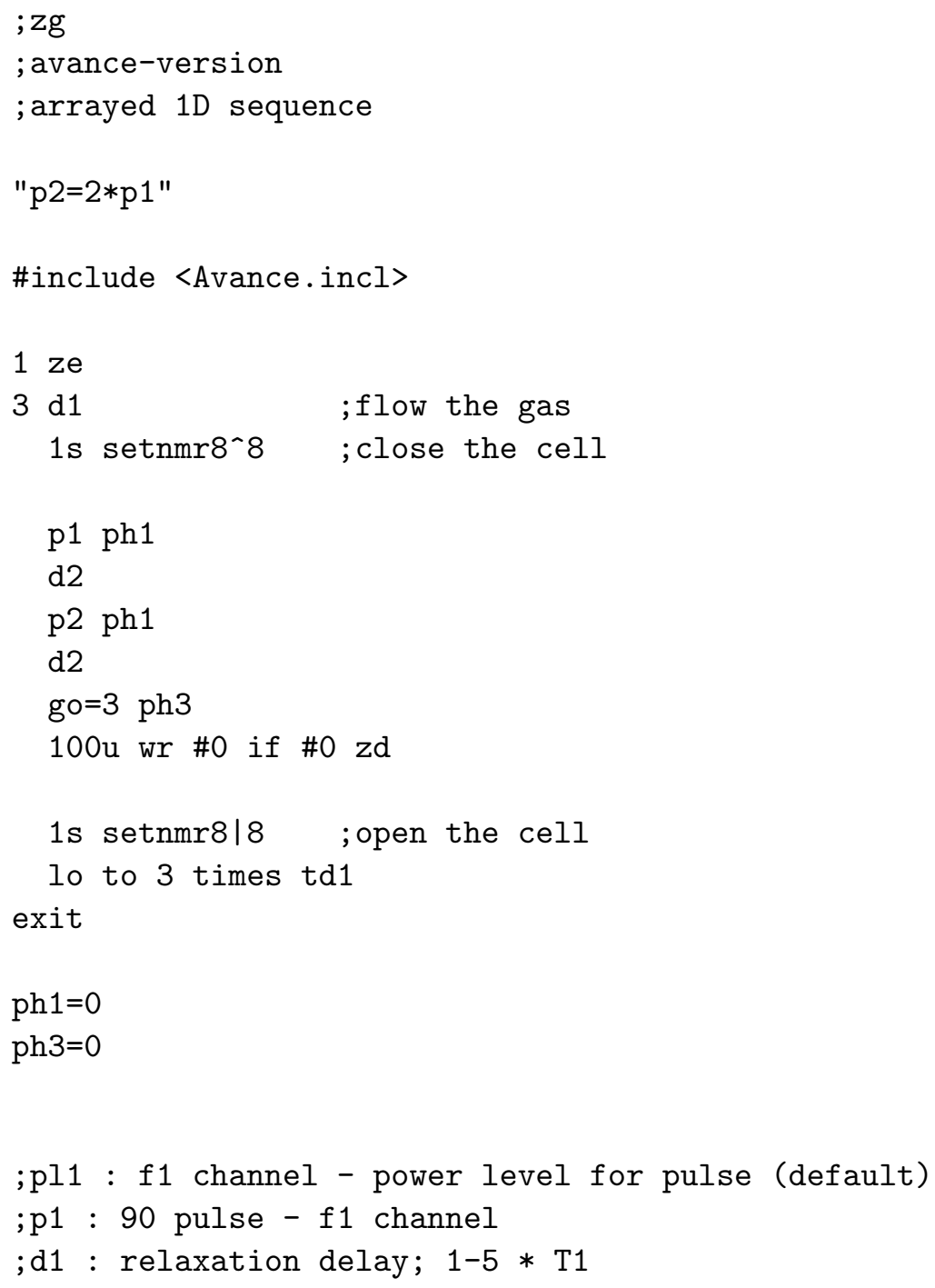

\section{B.1.2 Bubbling Setup}

Several experimental details are important here. After bubbling with $\mathrm{H}_{2}$, the solution is saturated with $\mathrm{H}_{2}$ gas, which continues to diffuse and react with the homogeneous or heterogeneous catalyst. This drawn out reaction time can be mitigated somewhat by bubbling a short burst of $\mathrm{N}_{2}$ after the initial $\mathrm{H}_{2}$ burst. Furthermore, if a heterogeneous 
Automated bubbling acquisition

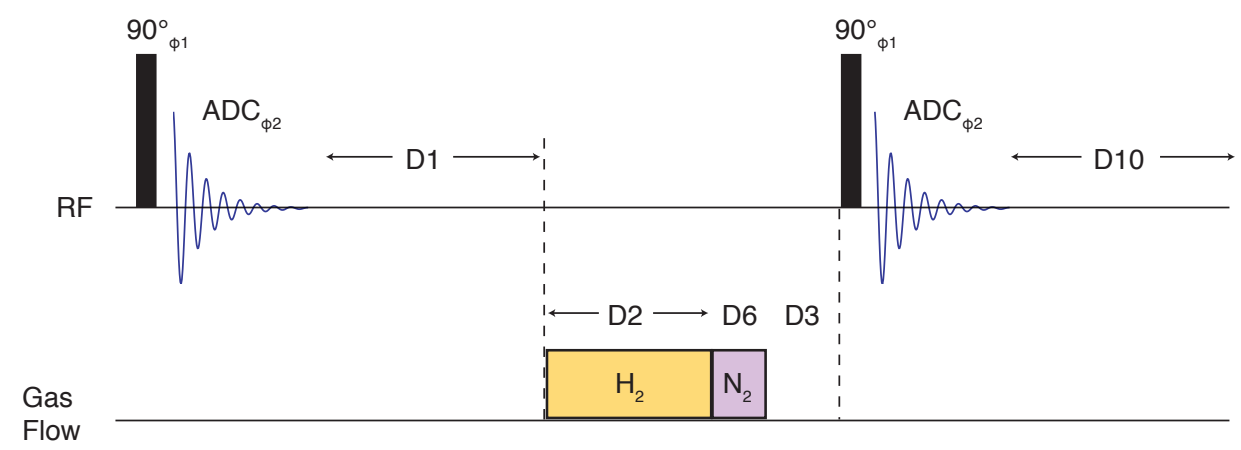

Figure B.2: The first acquisition is to acquire the thermal background, e.g., if any built up product needs to be subtracted from the polarized spectrum. After $\mathrm{H}_{2}$ bubbling, the cell is purged with $\mathrm{N}_{2}$ and the short delay allows everything to settle before the final acquisition.

catalyst is being used, the particles are stirred up by the bubbling and cause severe susceptibility distortions. A short delay is needed after bubbling to allow the beads to settle while the low order shims are quickly adjusted by hand before acquisition. Finally, there are problems with the bubbling process itself. If the bubbling is too violent (i.e. the pressure is too high), the contents of the cells are violently ejected into the exhaust tube, but if the pressure is too low, there is a delay before the bubbling starts and the mixing is poor. A simple approach is to use a high enough pressure for good mixing, but cycle the vent switch so the back pressure of the $\mathrm{H}_{2}$ gas is approximately 1atm before bubbling.

The following code is for the Bruker Avance spectrometer using xWinNMR or TopSpin.

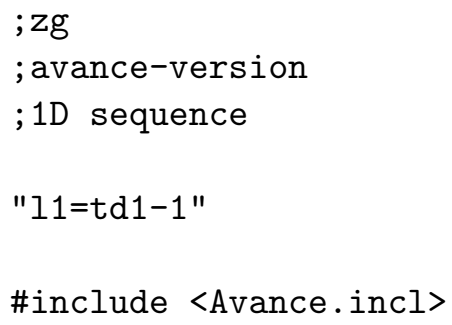




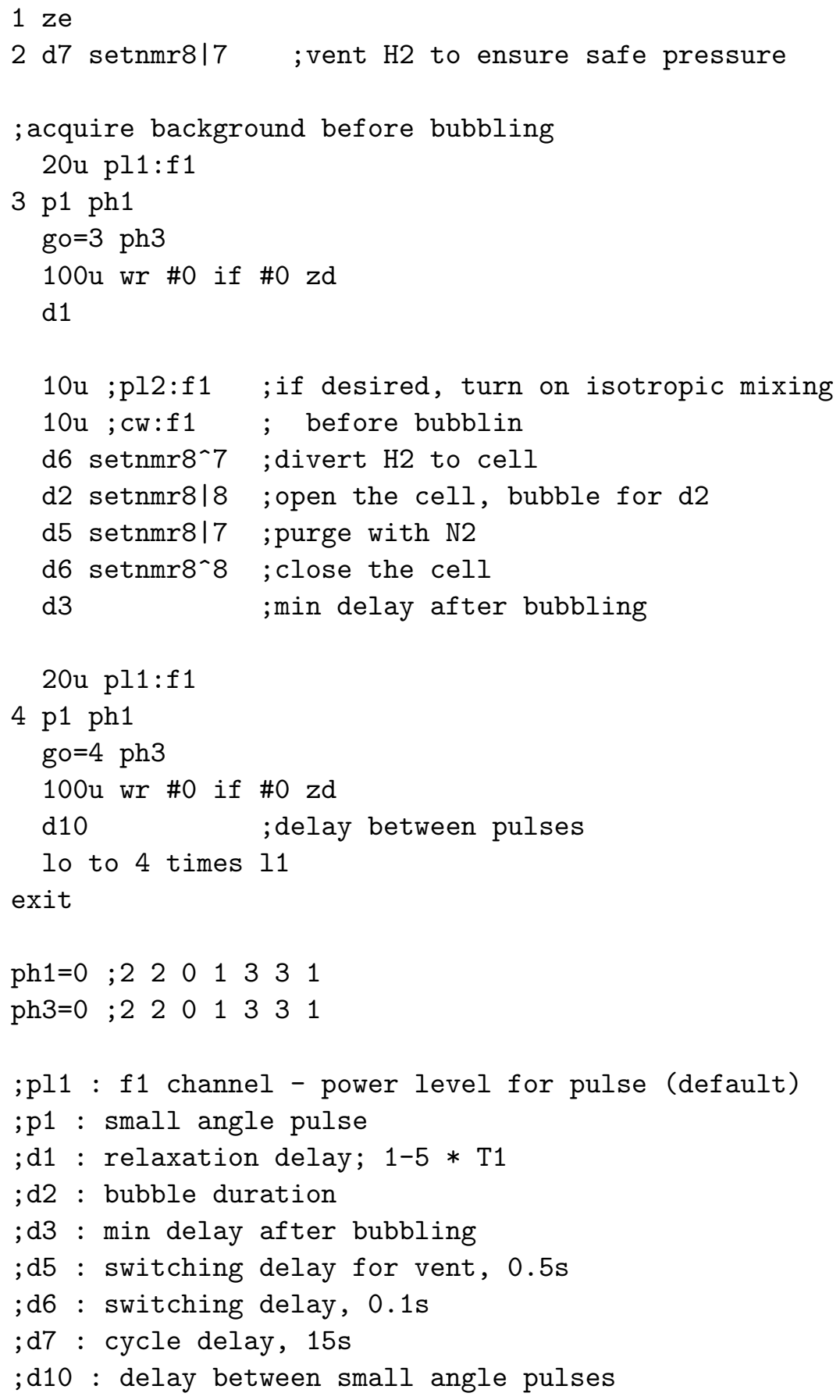


Spectroscopic imaging

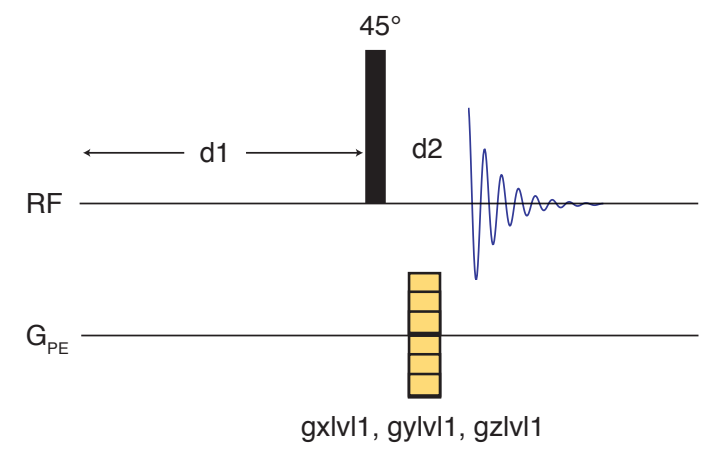

Figure B.3: A single point in k-space is acquired by turning the gradients on during the spin echo and only the top of the echo is recorded. This sets the resolution limit to the homogeneous line broadening, which, for a gas, is dominated by diffusion during the encoding time.

\section{B.1.3 $p-\mathrm{H}_{2}$ Polarized Void Space Imaging}

To image $p-\mathrm{H}_{2}$ polarized gas, the principle adjustment required is the flip angle, to optimize the signal strength and form of the signal. This is typically $45^{\circ}$ for PASADENA spectra, but can be $90^{\circ}$ for ALTADENA spectra, the specific flip angle depends on the J-coupling topology. It should be noted that the short $\mathrm{T}_{2}^{*}$ (approx. 10ms) will significantly reduce the resolution in the direct read out direction. Furthermore, the susceptibility differences of the bulk gas, the phantom and the glass of the NMR tube will cause significant distortion of the image due to offset effects. This is largely avoided by using single point imaging (SPI), also known as pure phase encoded imaging. By acquiring k-space in a point by point fashion, a spin echo is used to refocus inhomogeneous broadening and any evolution under offset effects become a simple attenuation factor. This attenuation factor will be spatially dependent, and thus, will affect the resulting image, but the severe distortions that occur during the direct readout are avoided, as explained in sec. 3.1.1. Because the 
resolution is dominated by the extent of k-space sampled, a large number of experiments are required to obtain high resolution images. For this reason, it is important to note that we are flowing a polarized gas into the void space, which means our repetition time $(T R)$ is not limited by $T_{1}$, but is set by the flow rate. This can allow for relatively fast traversal of k-space compared to standard SPI acquisitions.

The following code is for a Varian Inova spectrometer using vnmr.

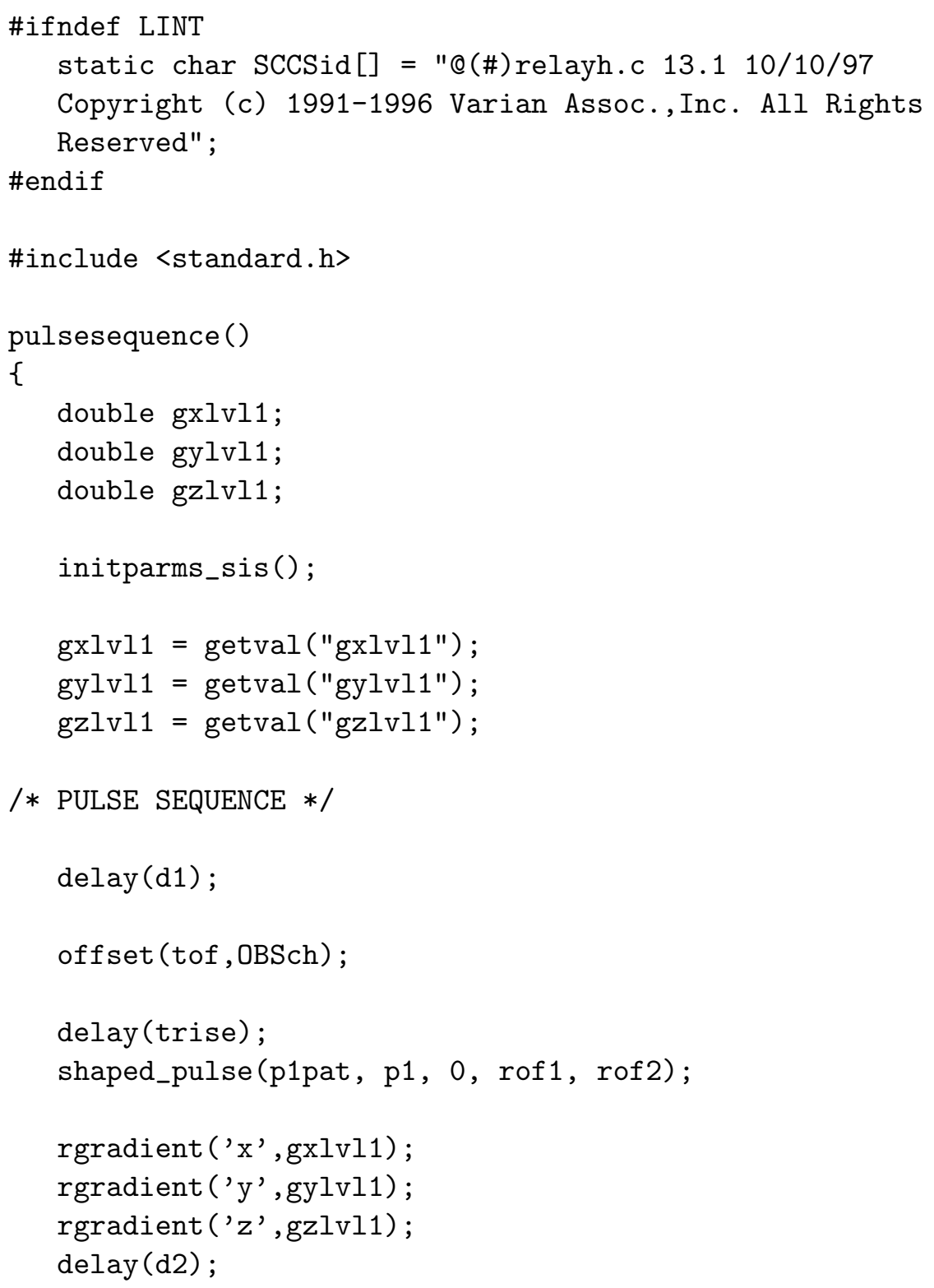




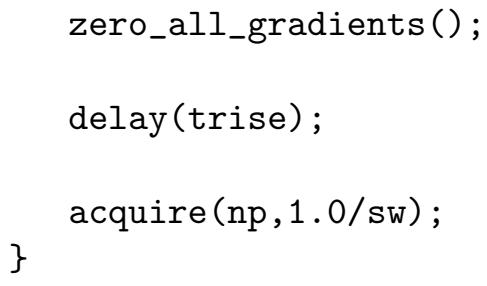

\section{B.1.4 Motion Compensated Pulse Sequences for Micro-reactor Imaging}

$$
\text { Spectroscopic imaging with velocity encoding }
$$

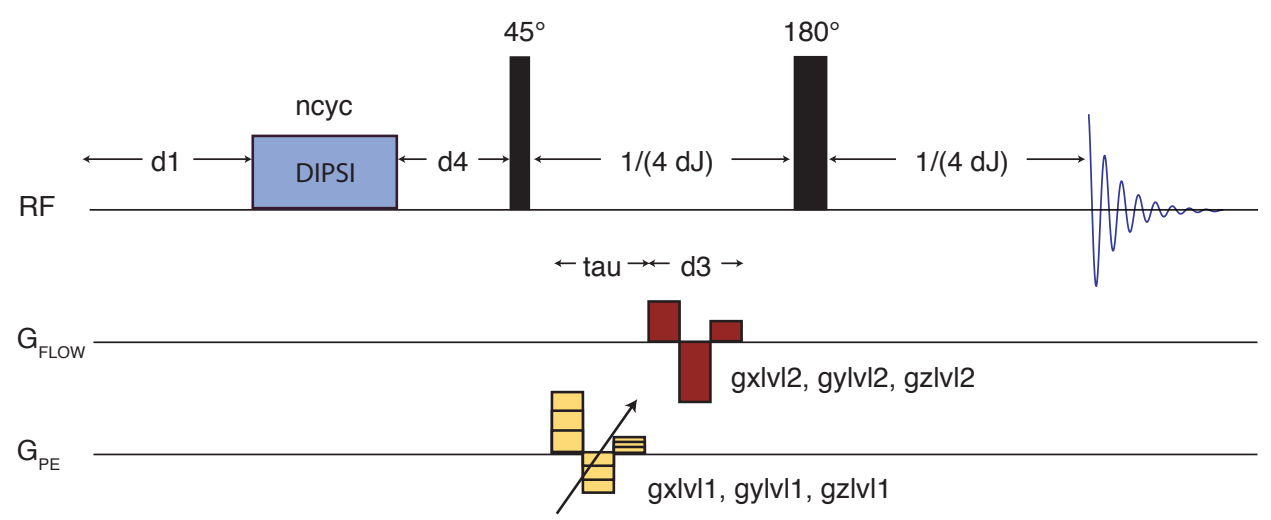

Figure B.4: A $45^{\circ}$ pulse is used to maximize the signal intensity of the PASADENA state. A motion compensated gradient pulse that is sensitive to position is applied at the beginning of the spin echo. If velocimetry measurements are desired, a gradient pulse that is sensitive only to velocity can be applied immediately after the position encoding pulse. A reference image is needed to extract the velocity dependent phase due to this pulse

Imaging PASADENA polarization is very similar to imaging ALTADENA polarization, as described above in sec. B.1.3. The key differences are the antiphase peaks and the fact that we are imaging gas in a bed of silica gel. The susceptibility broadening due to the silica gel results in large signal cancellation of the antiphase peaks (in the case of propane, $J \approx 15 \mathrm{~Hz}$ while the line width is $\sim O(100 \mathrm{~Hz})$. Luckily, the $180^{\circ}$ pulse used to refocus the inhomogeneous broadening can also serve as a rephasing sequence to turn the 
antiphase peaks into absorptive peaks, as described in sec. 5.1.2. Unlike the void space imaging in sec. B.1.3, the bulk motion of the gas is perpendicular to the slice thickness and this results in significant blurring and phase errors during encoding. To compensate for this motion, three-lobed gradient pulses are used that are sensitive only to the position or the velocity, as explained in sec. 4.4.6.

The following code is for a Varian Inova spectrometer using vnmr.

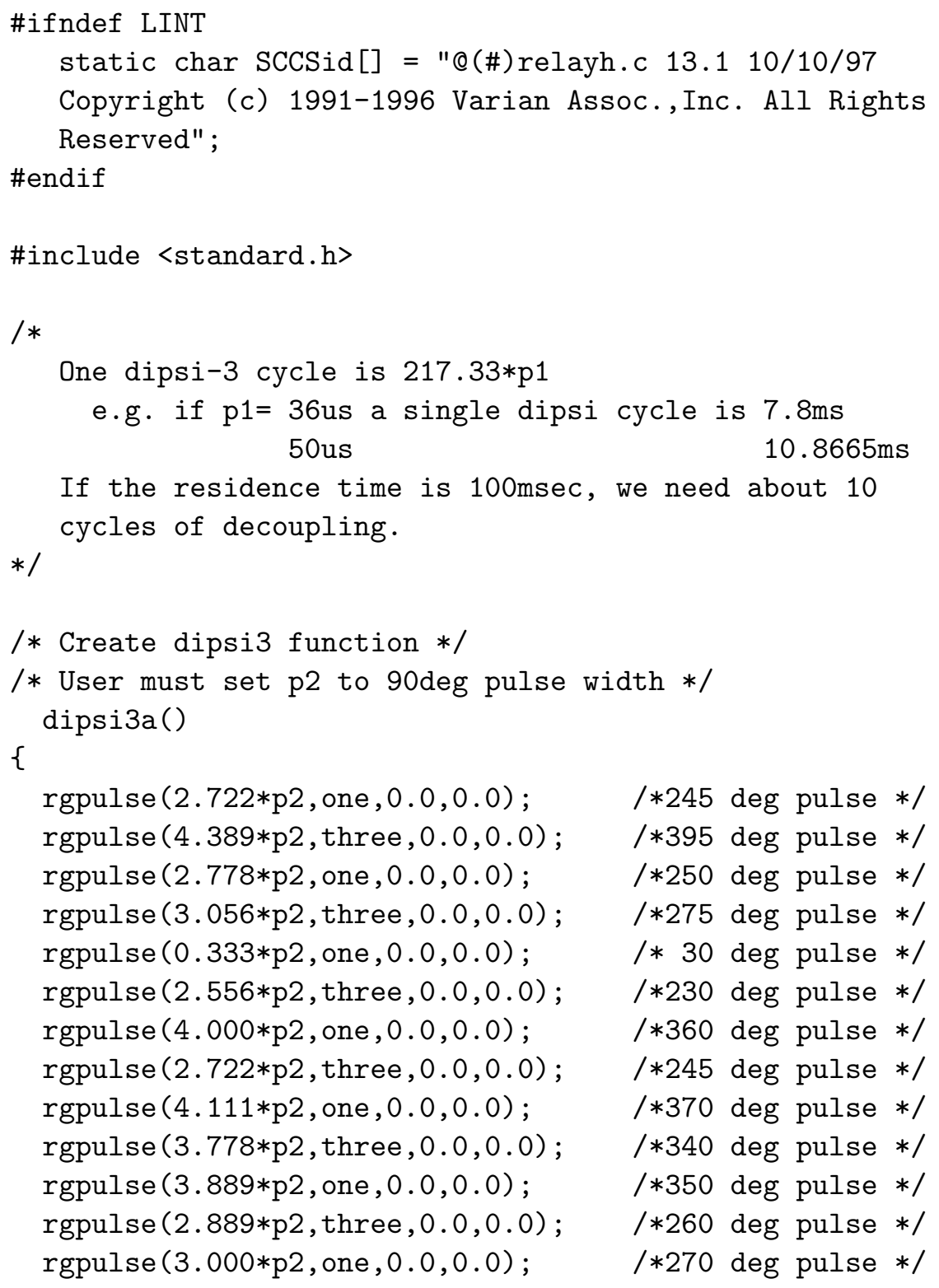




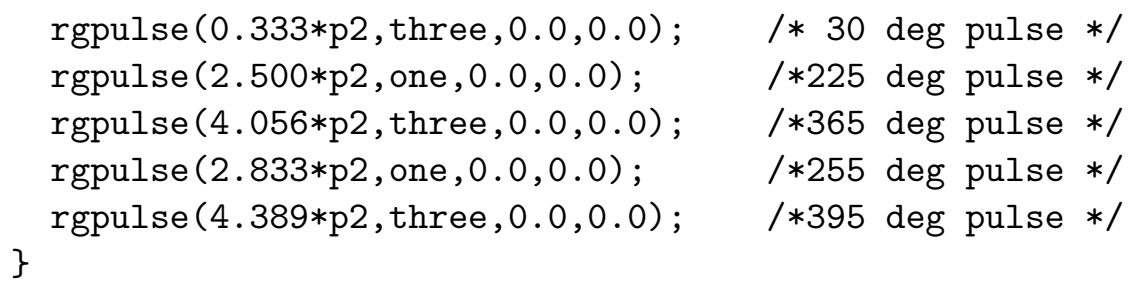




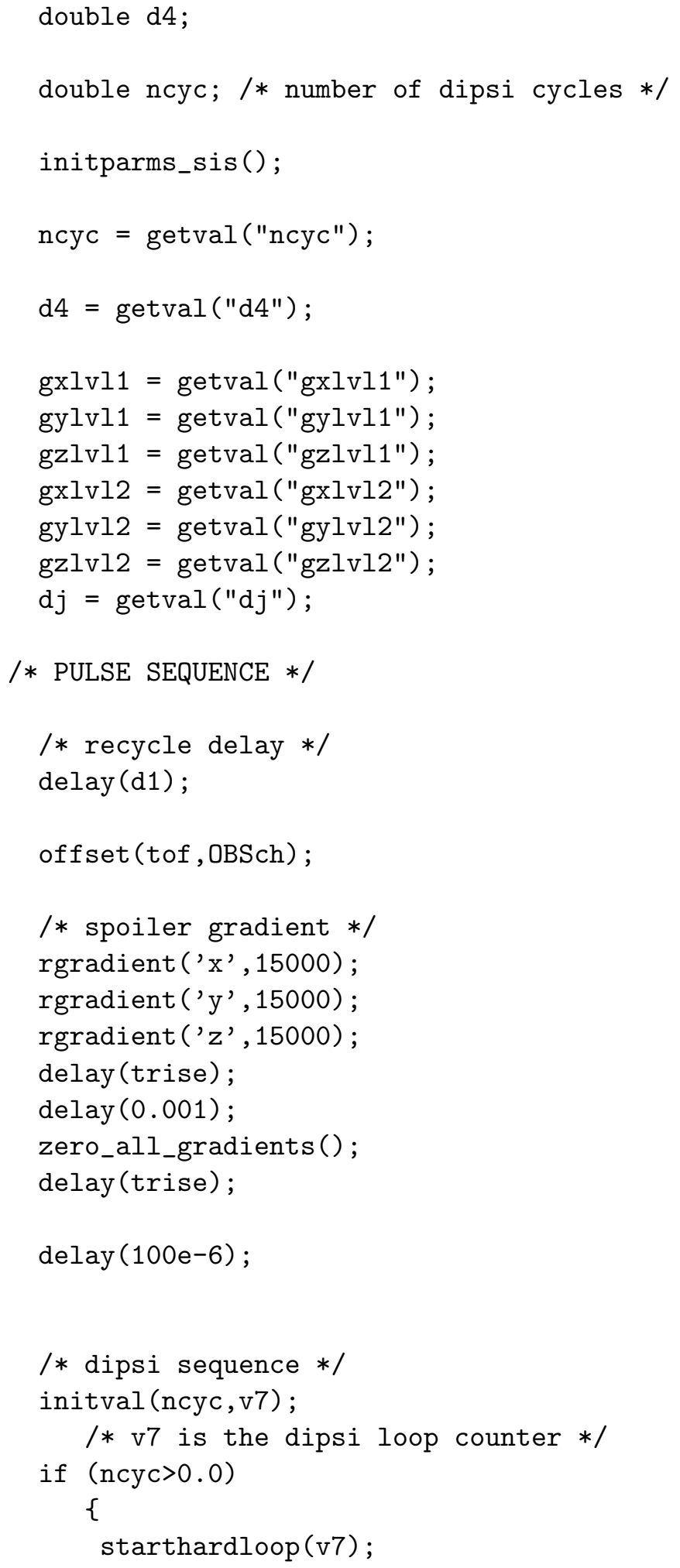




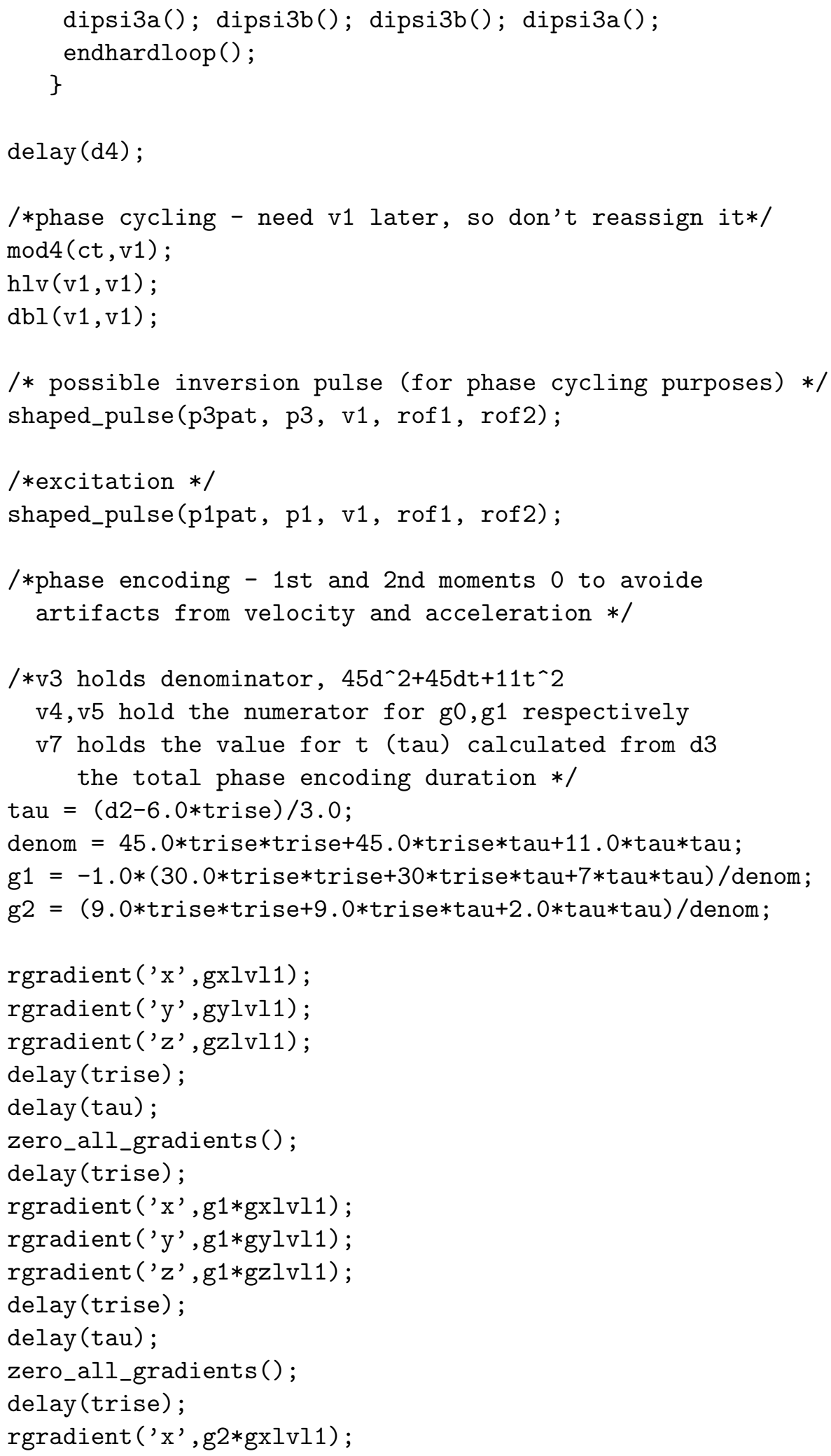




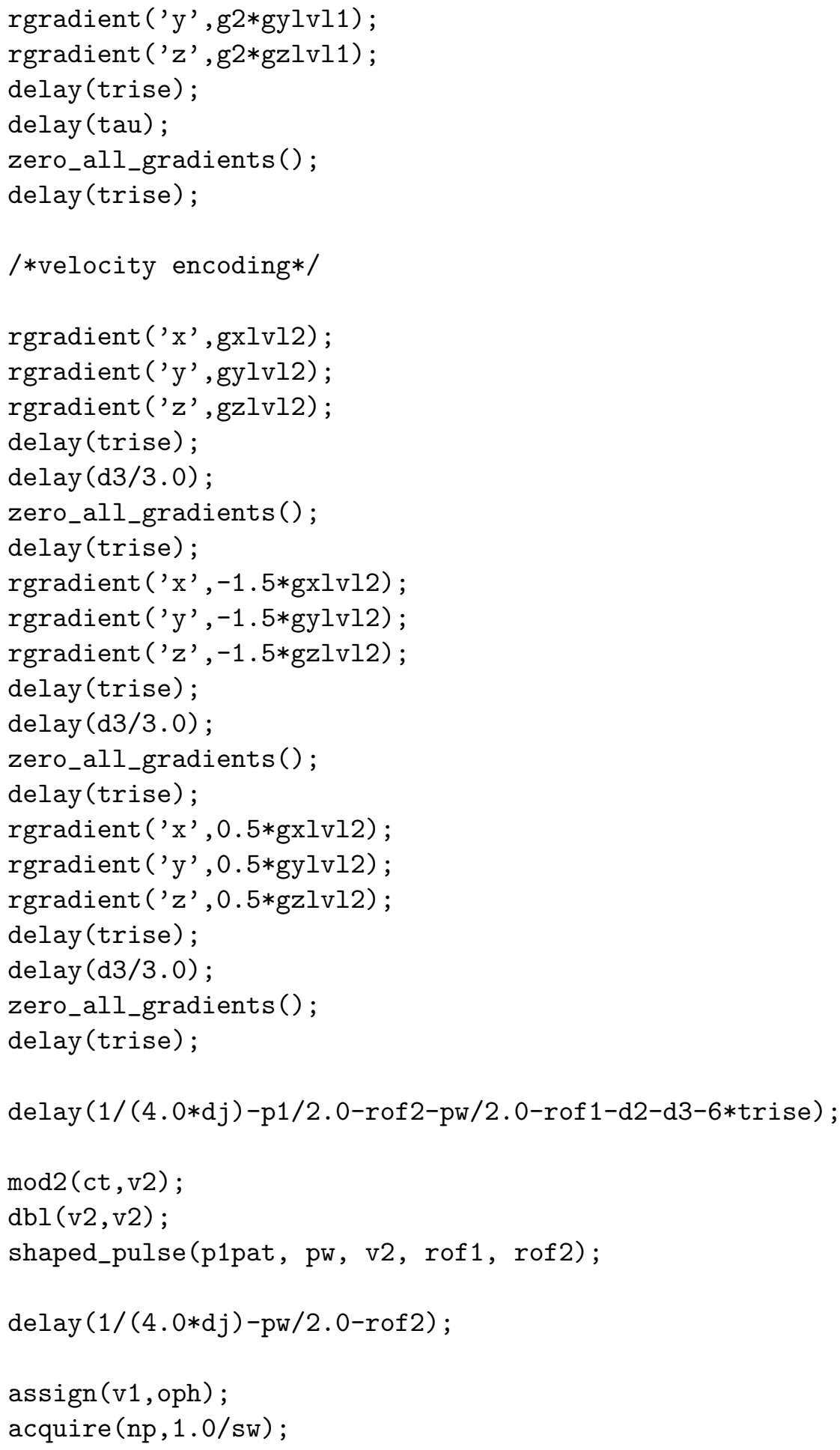




\section{B.2 Selected Matlab Code}

\section{B.2.1 Loading Spectrometer Data}

The Bruker data is quite easy to load as the ser file is simply the raw data.

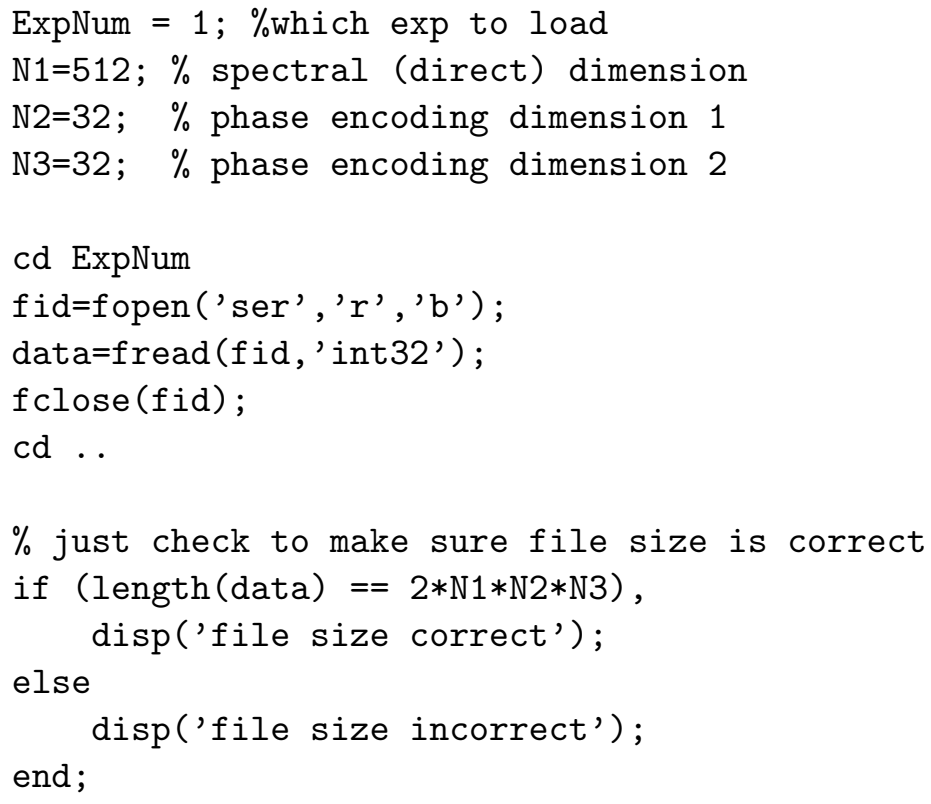

The Varian data requires a little bit of parsing,

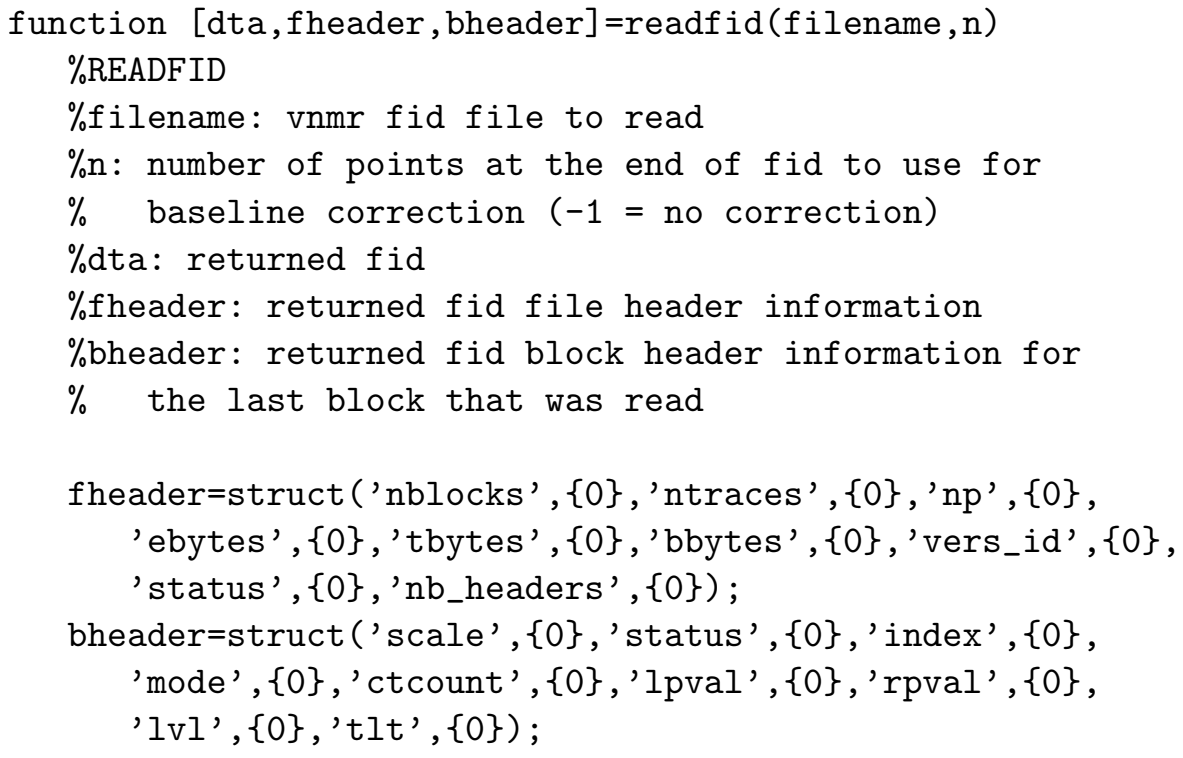




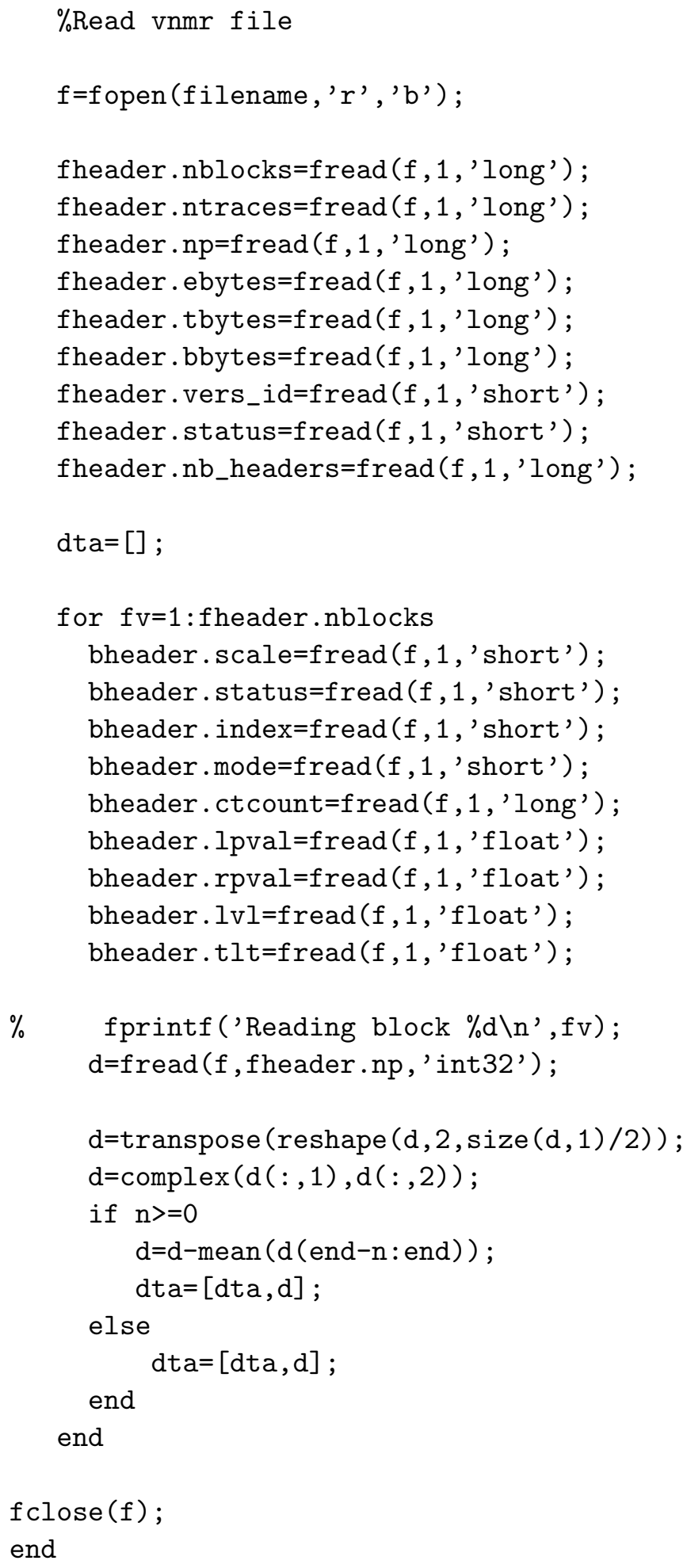




\section{B.2.2 Model Propane Spectrum for Temperature Mapping}

Chapter 5 presents a method of mapping the temperature distribution within the catalyst bed. This is done by fitting a combination of thermal and PASADENA spectra (eq. 5.1 and 5.2) to the CSI data. This is done using MATLAB's nlinfit algorithm,

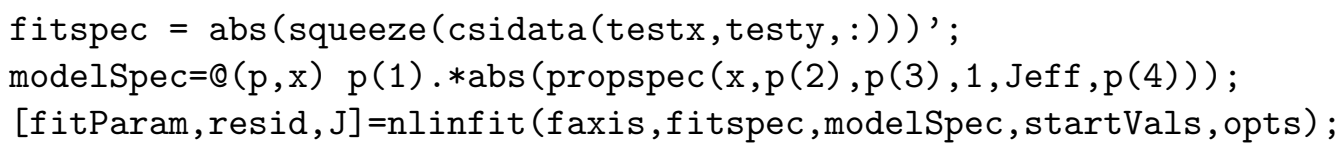

where csidata contains the spectra for each voxel in the micro-reactor, modelSpec in the simulated propane spectrum with the parameters being overall magnitude, frequency offset, line width and relative fraction of PASADENA signal to thermal signal, respectively.

The simulated spectrum, propspec.m, is simply a collection of lorentzian peaks at the correct positions for propane and phased according the parameter $J_{\text {eff }}=(2 T E)^{-1}$.

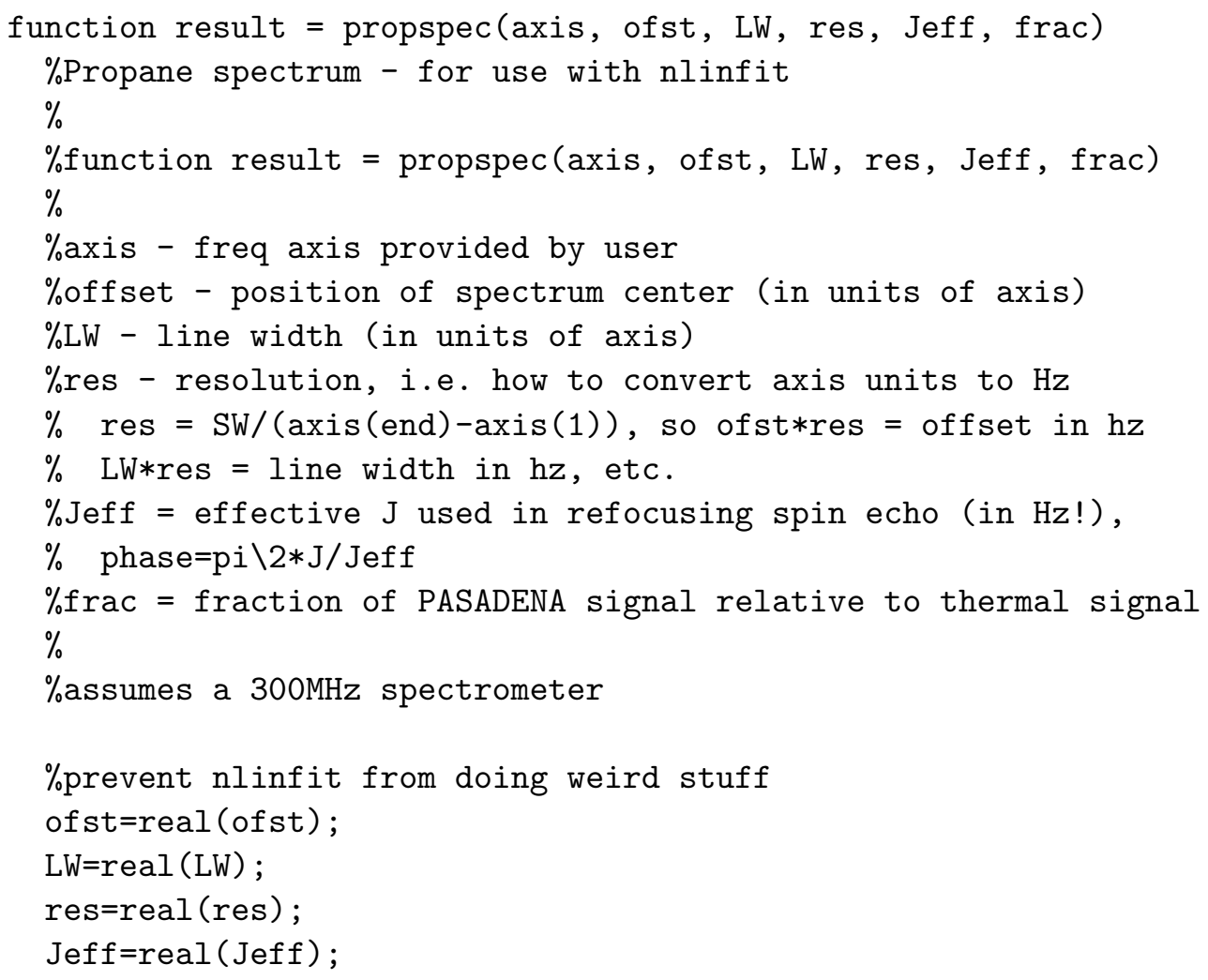




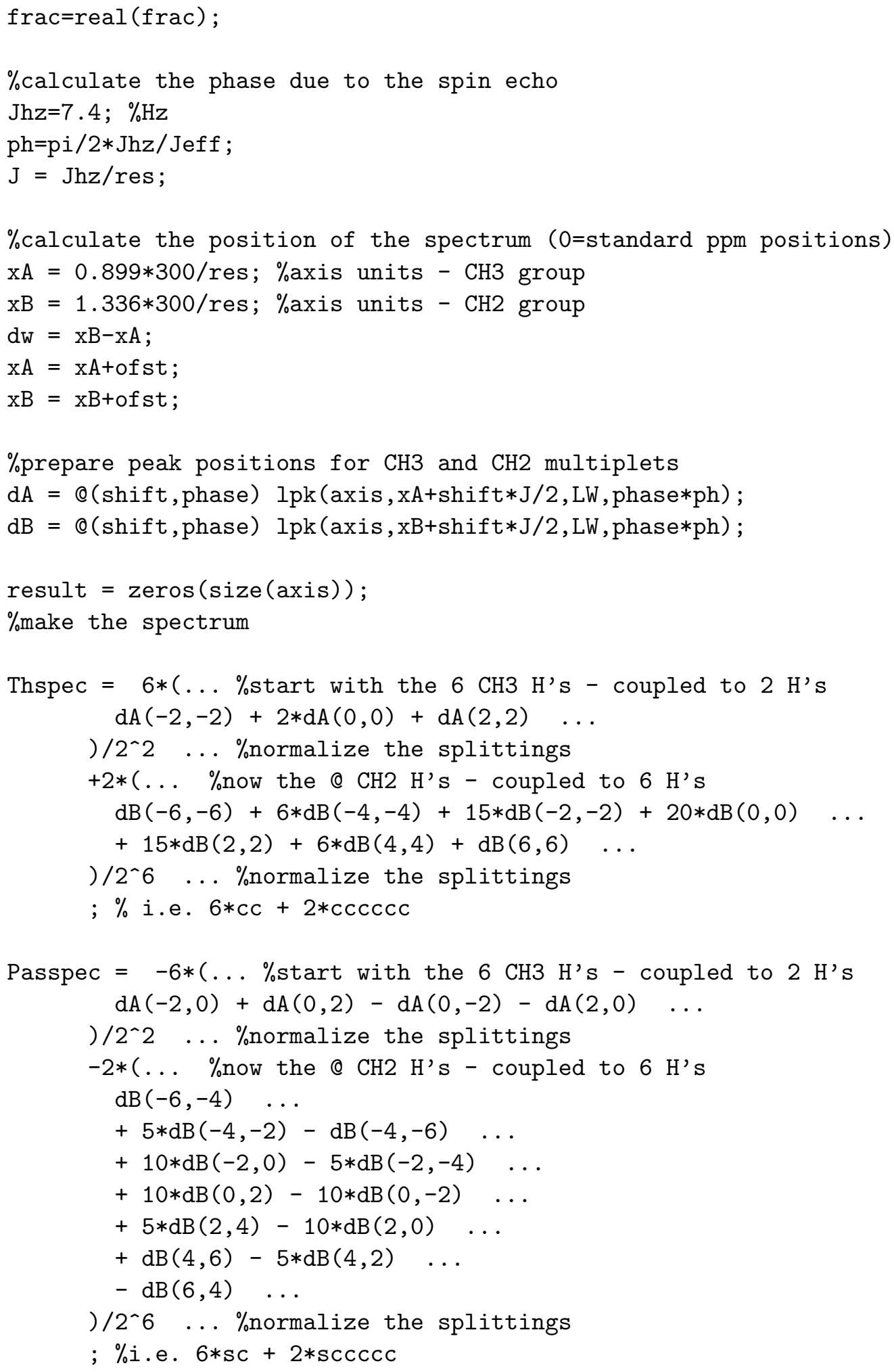


result $(:)=$ frac*Passpec+Thspec;
$\% \operatorname{result}(:)=$ frac*Passpec+(1-frac $) *$ Thspec;

And the lorentzian peak is given by lpk.m.

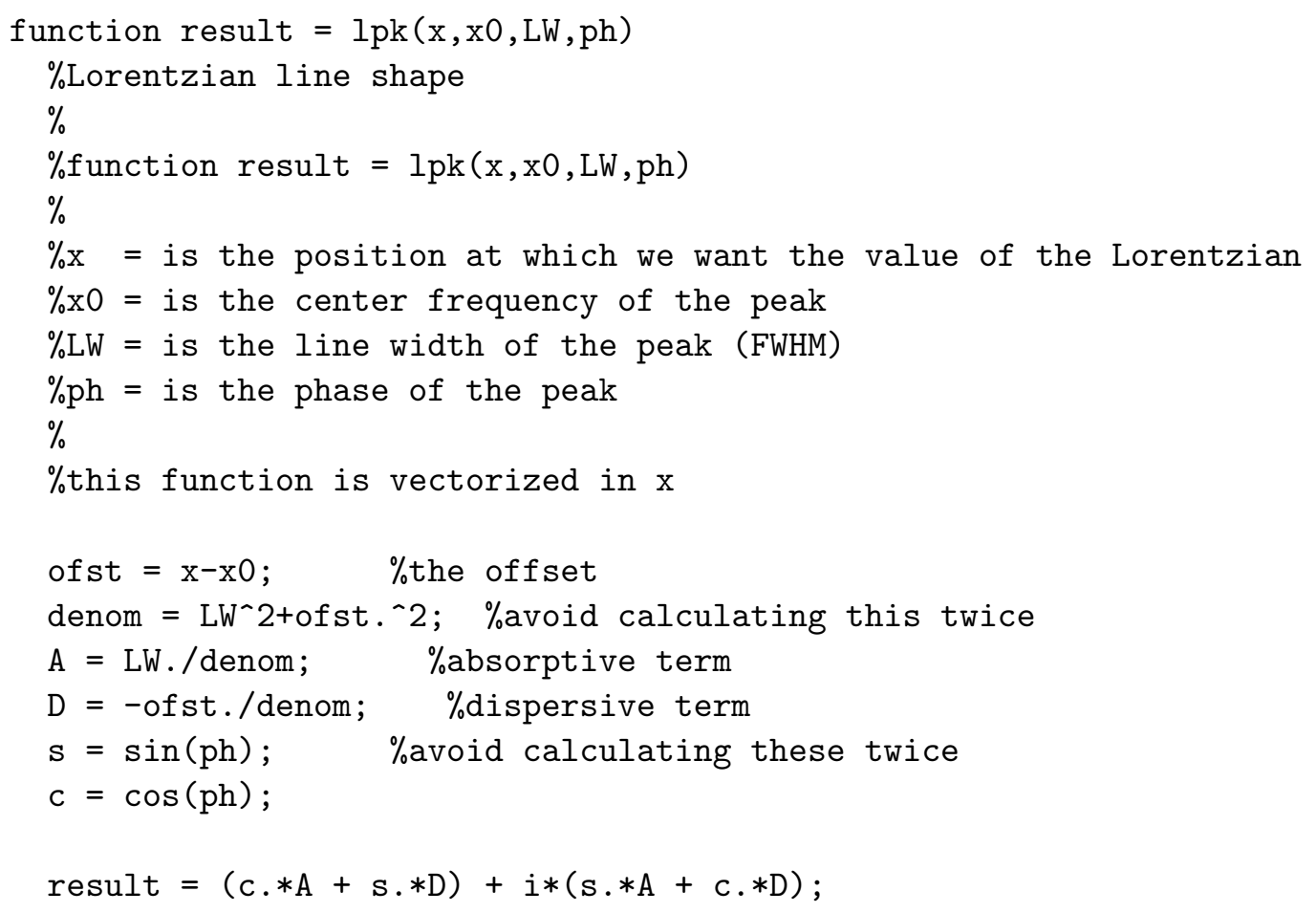

\section{B.2.3 Iterative Approach to Poisson Equation}

As discussed in ch. 6, a simple approach to solving the Laplace or Poisson equation is via an iterative approach based on a numerical approximation of the derivative. This approach is particularly nice when the empirical data already lies on a grid. The following matlab code performs the Gauss-Seidel iteration (with the possibility of successive over or under relaxation).

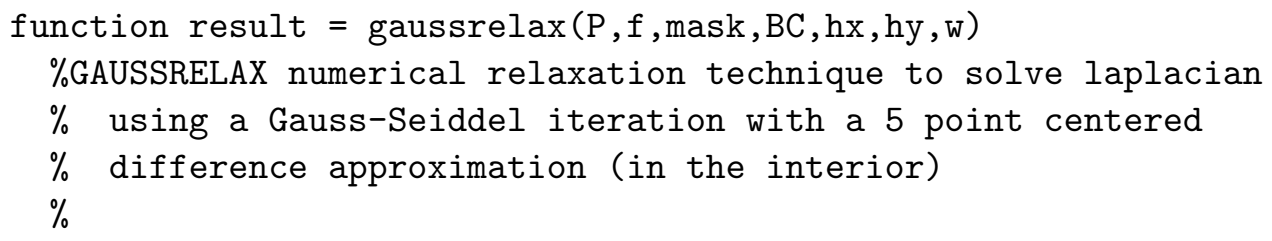




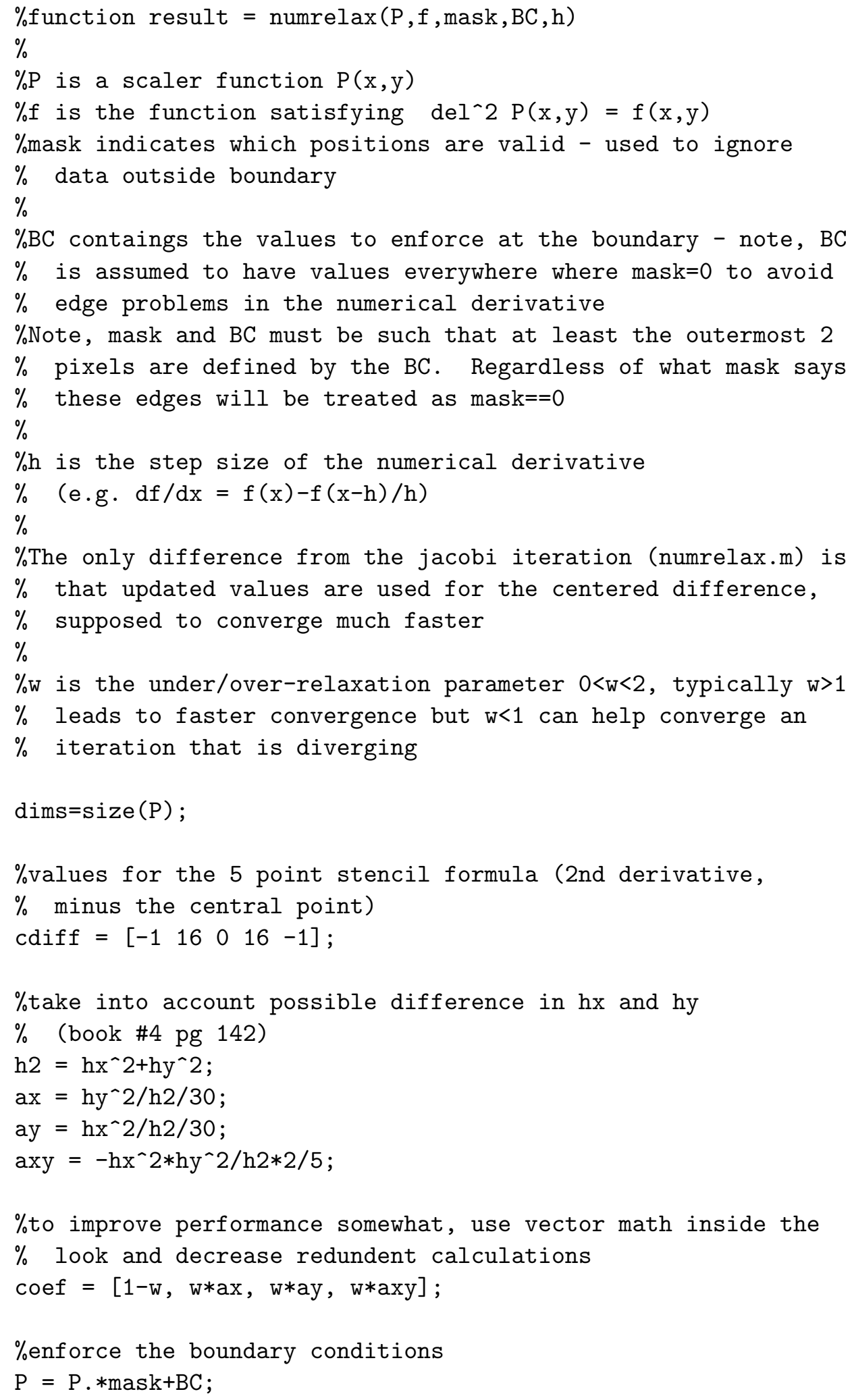




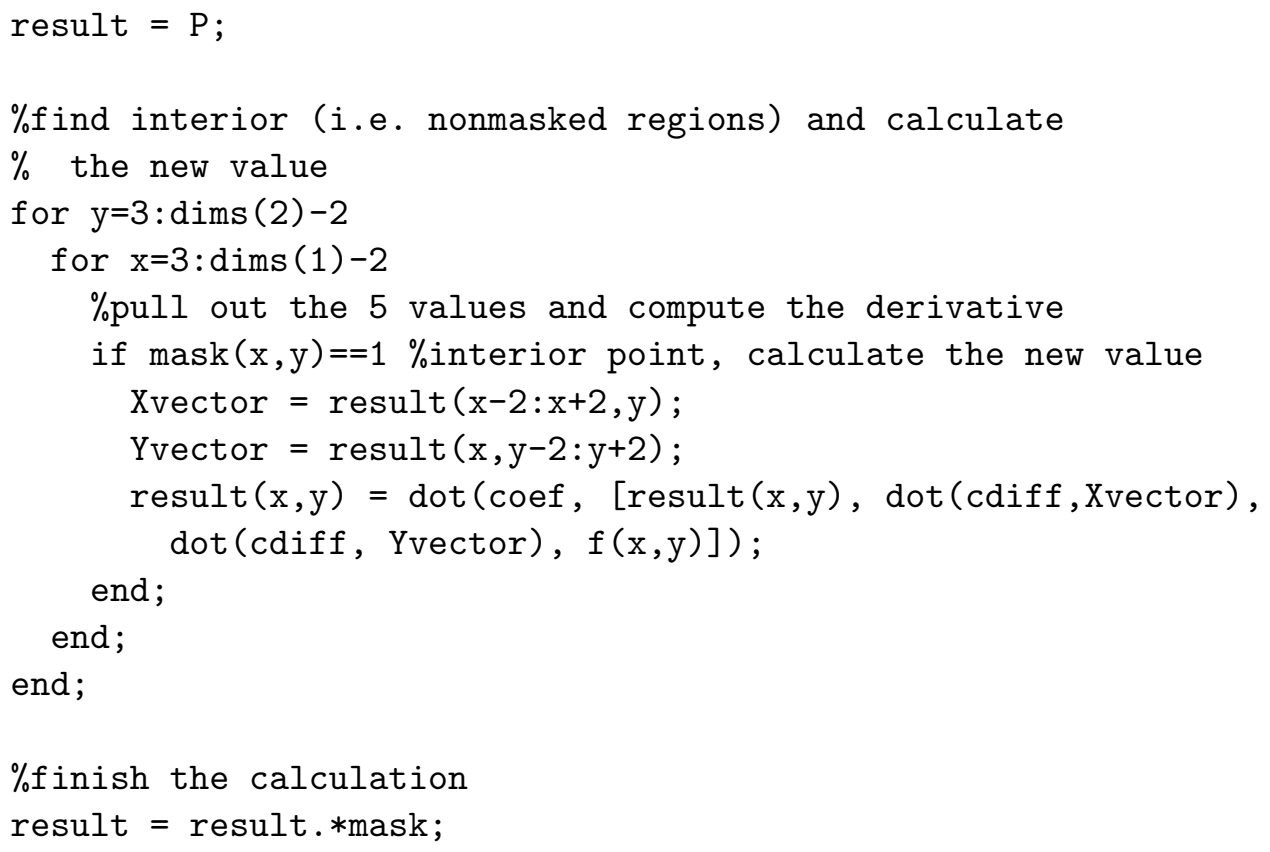

To calculate the source term for the Poisson equation, a numerical derivative is needed to take various derivatives of the velocity components (sec. 6.2).

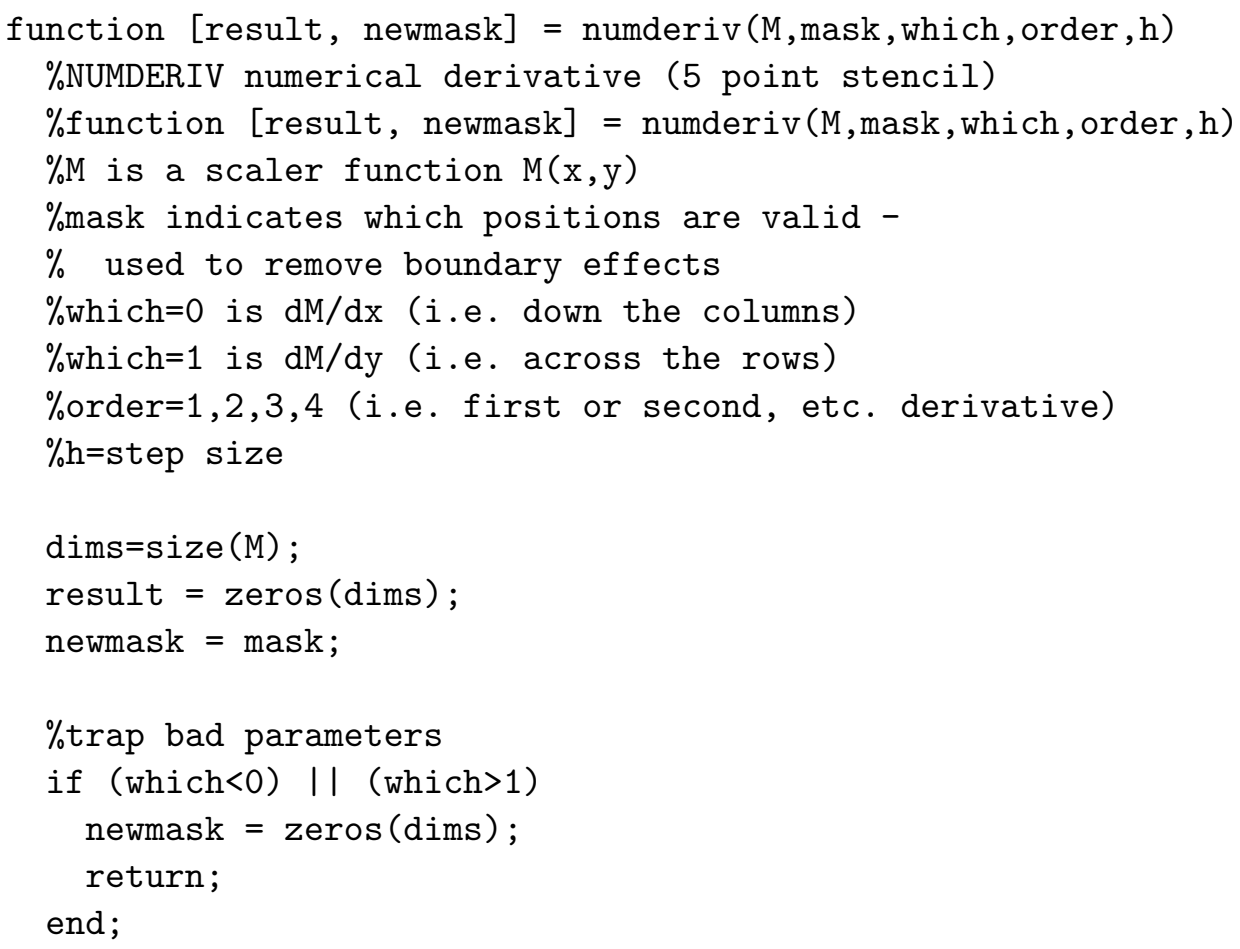




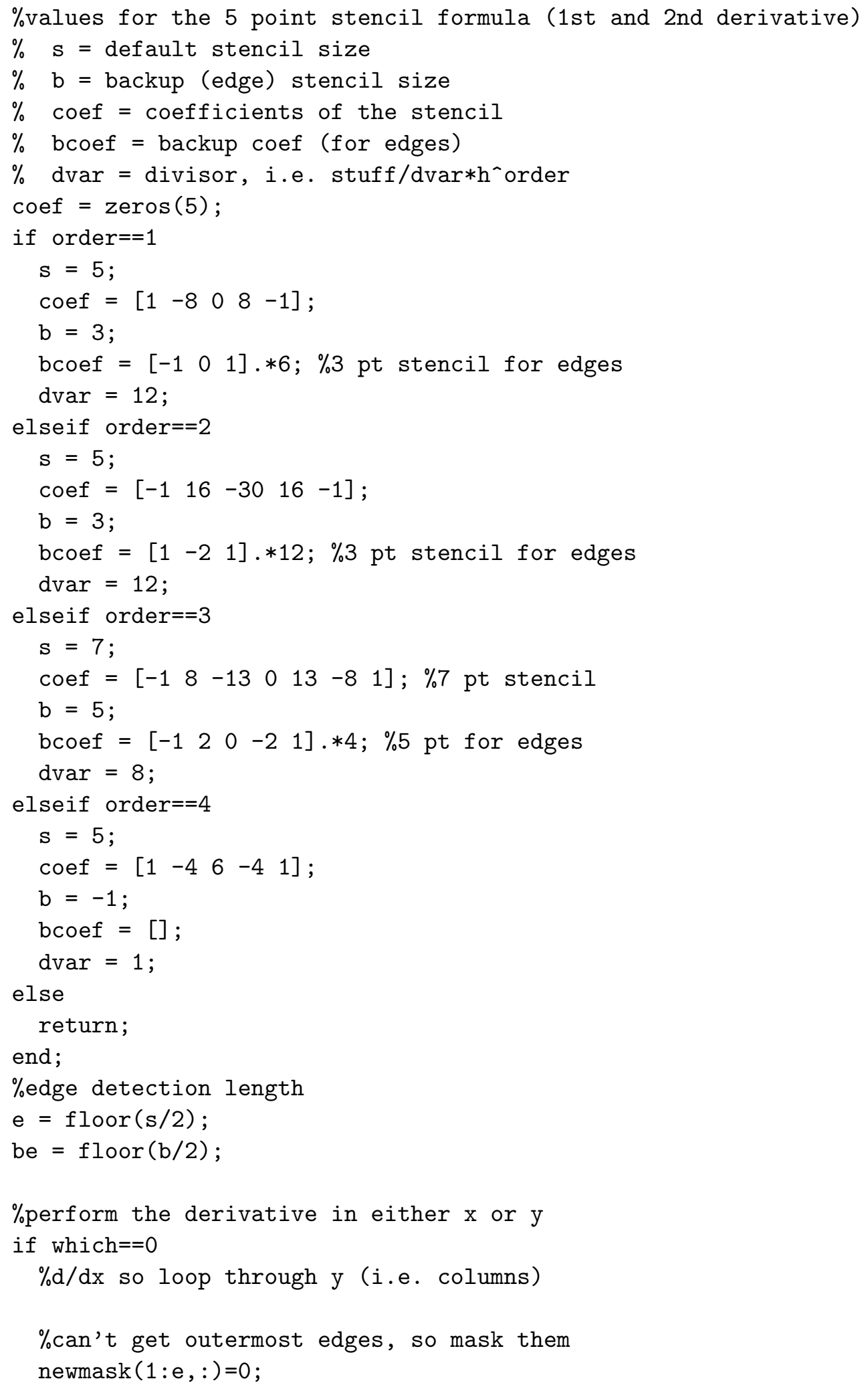




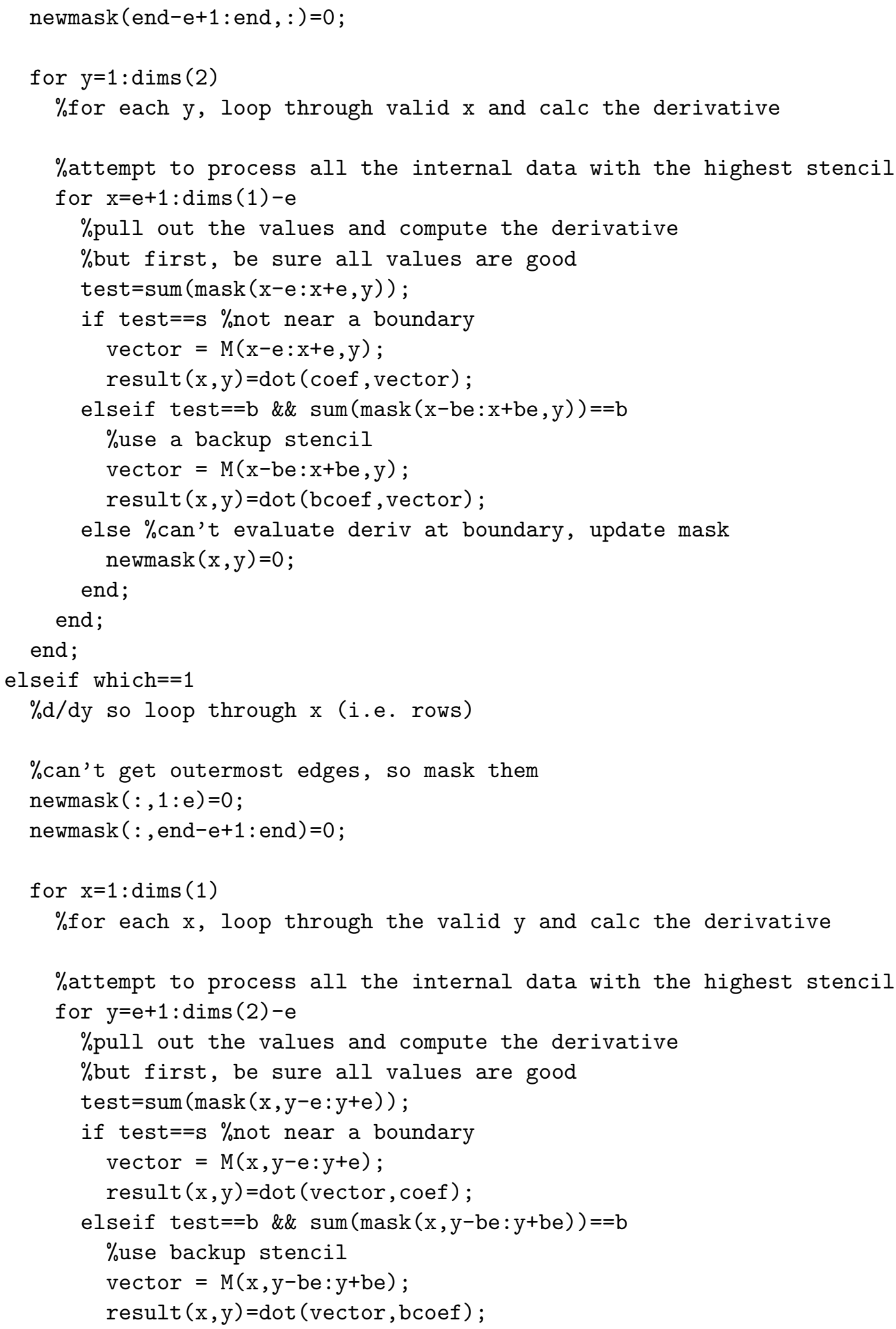


else \%can't evaluate deriv at boundary, update mask newmask $(x, y)=0$;

end;

end;

end;

end;

result $=$ result. $/($ dvar $*$ h`order $)$;

$\operatorname{disp}([' h=', \operatorname{num} 2 \operatorname{str}(h)])$; 


\section{Bibliography}

[1] A. Abragam. The Principles of Nuclear Magnetism. Oxford University Press, 1961.

[2] Milton Abramowit and Irene A. Stegun. Handbook of Mathematical Functions with Formulas, Graphs, and Mathematical Tables. Dover, 1970.

[3] Juan A. Aguilar, Paul I. P. Elliott, Joaquin Lopez-Serrano, Ralph W. Adams, and Simon B. Duckett. Only para-hydrogen spectroscopy (OPSY), a technique for the selective observation of para-hydrogen enhanced NMR signals. Chemical Communications, pages 1183-1185, 2006.

[4] Mårten Ahlquist, Mikaela Gustafsson, Magnus Karlsson, Mikkel Thaning, Oskar Axelsson, and Ola F. Wendt. Rhodium(I) Hydrogenation in Water: Kinetic Studies and the Detection of an Intermediate Using 13C1H PHIP NMR Spectroscopy. Inorganica Chimica Acta, 360(5):1621-1627, April 12007.

[5] Batoul Ahmed-Omer, Johan C. Brandt, and Thomas Wirth. Advanced Organic Synthesis Using Microreactor Technology. Organic and Biomolecular Chemistry, 5:733$740,2007$. 
[6] Susanna Ahola, Federico Casanova, Juan Perlo, Kerstin Münnemann, Bernhard Blümich, and Siegfried Stapf. Monitoring of Fluid Motion in a Micromixer by Dynamic NMR Microscopy. Lab on a Chip, 6(1):90-95, 2006.

[7] Silvio Aime, Walter Dastrü, Roberto Gobetto, and Alessandra Viale. ParaHydrogenation of Unsaturated Moieties on Poly(lysine) Derived Substrates for the Development of Novel Hyperpolarized MRI Contrast Agents. Organic and Biomolecular Chemistry, 3(21):3948-3954, 2005.

[8] Silvio Aime, Roberto Gobetto, and Daniel Canet. Longitudinal Nuclear Relaxation in an A2 Spin System Initially Polarized through Para-Hydrogen. Journal of the American Chemical Society, 120(27):6770-6773, July 151998.

[9] Silvio Aime, Roberto Gobetto, Francesca Reineri, and Daniel Canet. Hyperpolarization Transfer from Parahydrogen to Deuterium via Carbon-13. Journal of Chemical Physics, 119(17):8890-8896, November 12003.

[10] M. Sabieh Anwar. NMR Quantum Information Processing using Para-Hydrogen. PhD thesis, University of Oxford, 2004.

[11] M. Sabieh Anwar, Damir Blazina, Hilary A. Carteret, Simon B. Duckett, T. K. Halstead, Jonathan A. Jones, C. M. Kozak, and R. J. K. Taylor. Preparing High Purity Initial States for Nuclear Magnetic Resonance Quantum Computing. Physical Review Letters, 93(4):040501, 2004.

[12] M. Sabieh Anwar, Christian Hilty, Chester Chu, Louis-S. Bouchard, Kimberly L. Pierce, and Alexander Pines. Spin Coherence Transfer in Chemical Transformations 
Monitored by Remote Detection NMR. Analytical Chemistry, 79(7):2806-2811, April 12007.

[13] Christie Aroulanda, Larisa Starovoytova, and Daniel Canet. Longitudinal Nuclear Spin Relaxation of Ortho- and Para-Hydrogen Dissolved in Organic Solvents. Journal of Physical Chemistry A, 111(42):10615-10624, October 252007.

[14] Abdurrahman Ç. Atesin, Simon B. Duckett, Christine Flaschenriem, William W. Brennessel, and Richard Eisenberg. Diastereoselective Oxidative Addition of Dihydrogen to $\operatorname{IrI}(\mathrm{CO})((\mathrm{R})-\mathrm{BINAP})$ and $[\operatorname{Ir}(\mathrm{CO}) 2((\mathrm{R})-\mathrm{BINAP})][\mathrm{SbF} 6]$. Inorganic Chemistry, 46(4):1196-1204, February 192007.

[15] Pierluigi Barbaro, Claudio Bianchini, Vladimiro Dal Santo, Andrea Meli, Simonetta Moneti, Rinaldo Psaro, Adriana Scaffidi, Laura Sordelli, and Francesco Vizza. Hydrogenation of Arenes over Silica-Supported Catalysts That Combine a Grafted Rhodium Complex and Palladium Nanoparticles: Evidence for Substrate Activation on Rh(single-site)-Pd(metal) Moieties. Journal of the American Chemical Society, 128(21):7065-7076, May 312006.

[16] Pierluigi Barbaro, Claudio Bianchini, Giuliano Giambastiani, Werner Oberhauser, Laura Morassi Bonzi, Filippo Rossi, and Vladimiro Dal Santo. Recycling Asymmetric Hydrogenation Catalysts by Their Immobilisation onto Ion-Exchange Resins. Dalton Transactions, 2004(12):1783-1784, 2004.

[17] J. Bargon, J. Kandels, and K. Woelk. Angewandte Chemie, International Edition, 29:58, 1990. 
[18] R. Barret, M. Berr, T. F. Cha, J. Demme, J. Donat, J. Dongarr, V. Eijkhou, R. Poz, C. Romin, Van der Vors, and H. Templates for the Solution of Linear Systems: Building Blocks for Iterative Methods. SIAM, 2nd edition, 1994.

[19] Tobias Bauer, Shaibal Roy, Ruediger Lange, and Muthanna Al-Dahhan. Liquid Saturation and Gas-liquid Distribution in Multiphase Monolithic Reactors. Chemical Engineering Science, 60(11):3101-3106, June 2005.

[20] Jens Beck and Sven Unterberger. The behaviour of phosphorus in the flue gas during the combustion of high-phosphate fuels. Fuel, 85:1541-1549, 2006.

[21] Detlev Belder. Integrating Chemical Synthesis and Analysis on a Chip. Analytical and Bioanalytical Chemistry, 385(3):416-418, June 2006.

[22] P. Belton, B. Hills, and G. Webb. Advances in Magnetic Resonance in Food Science. Royal Society of Chemistry, 1999.

[23] P. Bhattacharya, Kent Harris, A. P. Lin, M. Mansson, Valerie A. Norton, W. H. Perman, Daniel P. Weitekamp, and Brian D. Ross. Ultra-FastTthree Dimensional Imaging of Hyperpolarized 13C in vivo. Magnetic Resonance Materials in Physics, Biology and Medicine, 18(5):245-256, October 2005.

[24] Claudio Bianchini and Pierluigi Barbaro. Recent Aspects of Asymmetric Catalysis by Immobilized Chiral Metal Catalysts. Topics in Catalysis, 19(1):17-32, March 2002.

[25] Claudio Bianchini, Daryl G. Burnaby, John Evans, Piero Frediani, Andrea Meli, Werner Oberhauser, Rinaldo Psaro, Laura Sordelli, and Francesco Vizza. Prepara- 
tion, Characterization, and Performance of Tripodal Polyphosphine Rhodium Catalysts Immobilized on Silica via Hydrogen Bonding. Journal of the American Chemical Society, 121(25):5961-5971, June 301999.

[26] Damir Blazina, Simon B. Duckett, John P. Dunne, and Cyril Godard. Applications of the Parahydrogen Phenomenon in Inorganic Chemistry. Dalton Transactions, 2004(17):2601-2609, 2004.

[27] Damir Blazina, Simon B. Duckett, Paul J. Dyson, and Joost A. B. Lohman. New Insights into Catalytic Hydrogenation by Phosphido-Substituted Triruthenium Clusters: Confirmation of Intact Cluster Catalysis by Parahydrogen NMR. Dalton Transactions, 2004(14):2108-2114, 2004.

[28] Damir Blazina, Simon B. Duckett, T. K. Halstead, C. M. Kozak, R. J. K. Taylor, M. Sabieh Anwar, Jonathan A. Jones, and Hilary A. Carteret. Generation and Interrogation of a Pure Nuclear Spin State by Parahydrogen-Enhanced NMR Spectroscopy: A Defined Initial State for Quantum Computation. Magnetic Resonance in Chemistry, 43(3):200-208, March 2005.

[29] Damir Blazina, John P. Dunne, Stuart Aiken, Simon B. Duckett, Charlotte Elkington, John E. McGrady, Rinaldo Poli, Sue J. Walton, M. Sabieh Anwar, Jonathan A. Jones, and Hilary A. Carteret. Contrasting Photochemical and Thermal Reactivity of $\mathrm{Ru}(\mathrm{CO}) 2(\mathrm{PPh} 3)$ (dppe) Towards Hydrogen Rationalised by Parahydrogen NMR and DFT Studies. Dalton Transactions, 2006(17):2072-2080, 2006.

[30] F. Bloch, W. W. Hansen, and M. E. Packard. Physical Review, 69:127, 1946. 
[31] Karl Blum. Density Matrix Theory and Applications. Physics of Atoms and Molecules. Plenum Press, 1981.

[32] Bernhard Blümich. NMR Imaging of Materials. Clarendon Press, 2000.

[33] Andrew R. Bogdan, Brian P. MAson, Kevin T. Sylvester, and D. Tyler McQuade. Improving Solid-Supported Catalyst Productivity by Using Simplified Packed-Bed Microreactors. Angewandte Chemie, International Edition, 46(10):1698-1701, February 262007.

[34] Louis-S. Bouchard, Scott R. Burt, M. Sabieh Anwar, Kirill V. Kovtunov, Igor V. Koptyug, and Alexander Pines. NMR Imaging of Catalytic Hydrogenation in Microreactors with the use of para-Hydrogen. Science, 319(5862):442-445, January 25 2008.

[35] Louis-S. Bouchard, Kirill V. Kovtunov, Scott R. Burt, M. Sabieh Anwar, Igor V. Koptyug, Renad Z. Sagdeev, and Alexander Pines. Para-hydrogen-enhanced hyperpolarized gas-phase magnetic resonance imaging. Angewandte Chemie, International Edition, 46(22):4064-4068, 2007.

[36] C. Russell Bowers. Sensitivity Enhancement Utilizing Parahydrogen. In D. M. Grant and R. K. Harris, editors, Encyclopedia of Nuclear Magnetic Resonance, volume 9 of Advances in NMR, pages 750-770. Wiley, Chichester, 2002.

[37] C. Russell Bowers and Daniel P. Weitekamp. Transformation of Symmetrization Order to Nuclear-Spin Magnetization by Chemical Reaction and Nuclear Magnetic Resonance. Physical Review Letters, 57(21):2645-2648, 1986. 
[38] C. Russell Bowers and Daniel P. Weitekamp. Parahydrogen and Synthesis Allow Dramatically Enhanced Nuclear Alignment. Journal of the American Chemical Society, 109(18):5541-5542, September 21987.

[39] John M. Brown, Phillip L. Evans, and Andrew R. Lucy. Stereochemistry of Intermediates in Homogeneous Hydrogenation Catalysed by Tristriphenylphosphinerhodium Chloride, Employing Nuclear Magnetic Resonance Magnetisation Transfer. Journal of the Chemical Society, Perkins Transactions 2, 1987(11):1589-1596, 1987.

[40] E. Brunner, Mathias Haake, L. Kaiser, Alexander Pines, and J. A. Reimer. Gas Flow MRI Using Circulating Laser-Polarized 129Xe. Journal of Magnetic Resonance, 138(1):155-159, May 1999.

[41] Eike Brunner. Enhancement of surface and biological magnetic resonance using laserpolarized noble gases. Concepts in Magnetic Resonance Part A, 11:313-335, 1999.

[42] G. Buntkowsky, B. Walaszek, A. Adamczyk, Y. Xu, H.-H. Limbach, and B. Chaudret. Mechanism of Nuclear Spin Initiated para-H2 to ortho-H2 Conversion. Physical Chemistry Chemical Physics, 8(16):1929-1935, 2006.

[43] Paul Callaghan. Principles of Nuclear Magnetic Resonance Microscopy. Oxford University Press, USA, 1994.

[44] Daniel Canet, Christie Aroulanda, Pierre Mutzenhardt, Silvio Aime, Roberto Gobetto, and Francesca Reineri. Para-Hydrogen Enrichment and Hyperpolarization. Concepts in Magnetic Resonance, 28(5):321-330, 2006. 
[45] Marina Carravetta, Ole G. Johannessen, and Malcolm H. Levitt. Beyond the T1 Limit: Singlet Nuclear Spin States in Low Magnetic Fields. Physical Review Letters, 92(15):153003, 2004

[46] Marina Carravetta and Malcolm H. Levitt. Long-Lived Nuclear Spin States in HighField Solution NMR. Journal of the American Chemical Society, 126(20):6228-6229, May 262004.

[47] Paul J. Carson, C. Russell Bowers, and Daniel P. Weitekamp. The PASADENA Effect at a Solid Surface: High-Sensitivity Nuclear Magnetic Resonance of Hydrogen Chemisorption. Journal of the American Chemical Society, 123(47):11821-11822, November 282001.

[48] John Cavanagh, Wayne J. Fairbrother, Arthur G. Palmer III, and Nicholas J. Skelton. Protein NMR Spectroscopy. Academic Press, 1st edition, 1996.

[49] Yunus A. Cengel and Michael A. Boles. Thermodynamics - An Engineering Approach. Mechanical Engineering. McGraw Hill, 3rd edition, 1989.

[50] Luisa Ciobanu, Dimuthu A. Jayawickrama, Xiaozhong Zhang, Andrew G. Webb, and Jonathan V. Sweedler. Measuring Reaction Kinetics by Using Multiple Microcoil NMR Spectroscopy. Angewandte Chemie, International Edition, 42(38):4669-4672, October 62003.

[51] Timothy D. W. Claridge. High-Resolution NMR Techniques in Organic Chemistry. Elsevier, 1st edition, 1999. 
[52] David J. Cole-Hamilton. Homogeneous Catalysis - New Approaches to Catalyst Separation, Recovery, and Recycling. Science, 299:1702-1706, March 142003.

[53] Jeffrey Cooper. Introduction to Partial Differential Equations with MATLAB. Birkhäuser, 2nd edition, 2000.

[54] Brian Cowan. Nuclear Magnetic Resonance and Relaxation. Cambridge University Press, 1997.

[55] Harold Craighead. Future Lab-on-a-chip Technologies for Interrogating Individual Molecules. Nature, 442(7101):387-393, 2006.

[56] Simon B. Duckett and Christopher J. Sleigh. Applications of the Parahydrogen Phenomenon: A Chemical Perspective. Progress in Nuclear Magnetic Resonance Spectroscopy, 34(1):71-92, February 151999.

[57] Dean G. Duffy. Transform Methods for Solving Partial Differential Equations. Chapman \& Hall, 2nd edition, 2004.

[58] John P. Dunne, Damir Blazina, Stuart Aiken, Hilary A. Carteret, Simon B. Duckett, Jonathan A. Jones, Rinaldo Poli, and Adrian C. Whitwood. A Combined Parahydrogen and Theoretical Study of H2 Activation by 16-Electron d8 Ruthenium(0) Complexes and Their Subsequent Catalytic Behaviour. Dalton Transactions, 2004(21):3616-3628, 2004.

[59] P. Earis. para-hydrogen to enhance MRI sensitivity. Journal of Analytical Atomic Spectrometry, 21(5):C35, 2006. 
[60] Andreas Eichhorn, Andreas Koch, and Joachim Bargon. In situ PHIP NMR - A New Tool to Investigate Hydrogenation Mediated by Colloidal Catalysts. Journal of Molecular Catalysis A: Chemical, 174(1-2):293-295, October 2001.

[61] Richard Eisenberg. Parahydrogen-Induced Polarization: A New Spin on Reactions with H2. Accounts of Chemical Research, 24:110-116, 1999.

[62] Thomas C. Eisenschmid, Rein U. Kirss, Paul P. Deutsch, Sven I. Hommeltoft, Richard Eisenberg, Joachim Bargon, Ronald G. Lawler, and Alan L. Balch. Para Hydrogen Induced Polarization in Hydrogenation Reactions. Journal of the American Chemical Society, 109(26):8089-8091, December 231987.

[63] R. R. Ernst and G. Bodenhausen. Principles of Nuclear Magnetic Resonance in One and Two Dimensions. Clarendon Press, Oxford, 1987.

[64] Daniel J. Fox, Simon B. Duckett, Christine Flaschenriem, William W. Brennessel, Jacob Schneider, Ahmet Gunay, and Richard Eisenberg. A Model Iridium Hydroformylation System with the Large Bite Angle Ligand Xantphos: Reactivity with Parahydrogen and Implications for Hydroformylation Catalysis. Inorganic Chemistry, 45(18):7197-7209, September 42006.

[65] Ray Freeman. Spin Choreography: Basic Steps in High Resolution NMR. Oxford University Press, Oxford, 1998.

[66] Cyril Godard, Simon B. Duckett, Céline Henry, Stacey Polas, Robert Toose, and Adrian C. Whitwood. New Perspectives in Hydroformylation : a para-Hydrogen Study. Chemical Communications, 2004(16):1826-1827, 2004. 
[67] Maurice Goldman and Haukur Jóhannesson. Conversion of a Proton Pair Para Order into 13C Polarization by rf Irradiation, for Use in MRI. Comptes Rendus Physique, 6(4-5):575-581, May-June 2005.

[68] Maurice Goldman, Haukur Jóhannesson, Oskar Axelsson, and Magnus Karlsson. Hyperpolarization of 13C Through Order Transfer from Parahydrogen: A New Contrast Agent for MRI. Magnetic Resonance Imaging, 23(2):153-157, February 2005.

[69] Maurice Goldman, Haukur Jóhannesson, Oskar Axelsson, and Magnus Karlsson. Design and Implementation of 13C Hyper Polarization from Para-Hydrogen, for New MRI Contrast Agents. Comptes Rendus Chimie, 9(3-4):357-363, March-April 2006.

[70] K. Golman, Oskar Axelsson, Haukur Jóhannesson, S. Månsson, C. Olofsson, and J. S. Petersson. Parahydrogen-Induced Polarization in Imaging: Subsecond 13C Angiography. Magnetic Resonance in Medicine, 46(1):1-5, July 2001.

[71] Boyd M. Goodson. Nuclear Magnetic Resonance of Laser-Polarized Noble Gases in Molecules, Materials, and Organisms. Journal of Magnetic Resonance, 155(2):157216, April 2002.

[72] Bernard III Gordon, Jacqueline S. Butler, and Ian R. Harrison. Rhodium(I) Catalyst Supported on Polymer Crystal Surfaces: Further Hydrogenation Studies. Journal of Polymer Science Part A: Polymer Chemistry, 25(8):2139-2142, August 1987.

[73] Josef Granwehr, Elad Harel, Song-I. Han, Sandra Garcia, Alexander Pines, P. N. Sen, and Y.-Q. Song. Time-of-Flight Flow Imaging Using NMR Remote Detection. Physical Review Letters, 95:075503, 2005. 
[74] Mathias Haake, Johannes Natterer, and Joachim Bargon. Efficient NMR Pulse Sequences to Transfer the Parahydrogen-Induced Polarization to Hetero Nuclei. Journal of the American Chemical Society, 118(36):8688-8691, September 111996.

[75] Jack Halpern and Chun Sing Wong. Hydrogenation of tris(triphenylphosphine)chlororhodium(I). Journal of the Chemical Society, Chemical Communications, 1973(17):629-630, 1973.

[76] Elad Harel, Christian Hilty, Katherine Koen, Erin E. McDonnell, and Alexander Pines. Time-of-Flight Flow Imaging of Two-Component Flow inside a Microfluidic Chip. Physical Review Letters, 98:017601, 2007.

[77] Stephen J. Haswell, Robert J. Middleton, Brian O'Sullivan, Victoria Skelton, Paul Watts, and Peter Styring. The Application of Micro Reactors to Synthetic Chemistry. Chemical Communications, 2001(5):391-398, 2001.

[78] Christian Hilty, Erin E. McDonnell, Josef Granswehr, Kimberly L. Pierce, Song-I. Han, and Alexander Pines. Microfluidic Gas-Flow Profiling Using Remote-Detection NMR. Proceedings of the National Academy of Sciences of the United States of America, 102(42):14960-14963, October 182005.

[79] J. Michael Hollas. Modern Spectroscopy. John Wiley \& Sons, 3rd edition, 1996.

[80] P. J. Hore, J. A. Jones, and S. Wimperis. NMR: The Toolkit. Oxford University Press, 2000. 
[81] D. I. Hoult. The Principle of Reciprocity in Signal Strength Calculations A Mathematical Guide. Concepts in Magnetic Resonance, 12(4):173-187, 2000.

[82] D. I. Hoult and B. Bhakar. NMR Signal Reception: Virtual Photons and Coherent Spontaneous Emission. Concepts in Magnetic Resonance, 9(5):277-297, 1998.

[83] D. I. Hoult and N. S. Ginsberg. The Quantum Origins of the Free Induction Decay Signal and Spin Noise. Journal of Magnetic Resonance, 148(1):182-199, 2001.

[84] L. Huang and S. Kawi. An Active and Stable RhH(CO)(PPh3)3-Derived SiO2Tethered Catalyst Via a Thiol Ligand for Cyclohexene Hydroformylation. Catalysis Letters, 90(3-4):165-169, October 2003.

[85] Andrea Ienco, Maria José Calhorda, Joachim Reinhold, Francesca Reineri, Claudio Bianchini, Maurizio Peruzzini, Francesco Vizza, and Carlo Mealli. Activation of Molecular Hydrogen over a Binuclear Complex with Rh2S2 Core: DFT Calculations and NMR Mechanistic Studies. Journal of the American Chemical Society, 126(38):11954-11965, September 292004.

[86] Haukur Jóhannesson, Oskar Axelsson, and Magnus Karlsson. Transfer of ParaHydrogen Spin Order into Polarization by Diabatic Field Cycling. Comptes Rendus Physique, 5(3):315-324, April 2004.

[87] E. Johansson, L.E. Olsson, S. Månsson, J.S. Petersson, K. Golman, F. Ståhlberg, and R. Wirestam. Perfusion Assessment With Bolus Differentiation: A Technique Applicable to Hyperpolarized Tracers. Magnetic Resonance in Medicine, 52:10431051, 2004. 
[88] Alfred Kaslter. uelques suggestions concernant la production optique et la détection optique d'une inégalité de population des niveaux de quantifigation spatiale des atomes. Application à l'expérience de Stern et Gerlach et à la résonance magnétique. Journal de Physique et Le Radium, 11(6):255-265, June 1950.

[89] Rainer Kimmich. NMR: Tomography, Diffusometry, Relaxometry. Springer, 1st edition, 2001.

[90] Juta Kobayashi, Yuichiro Mori, Kuniaki Okamoto, Ryo Akiyama, Masaharu Ueno, Takehiko Kitamori, and Shu Kobayashi. A Microfluidic Device for Conducting GasLiquid-Solid Hydrogenation Reactions. Science, 304(5675):1305-1308, May 282004.

[91] Igor V. Koptyug, Kirill V. Kovtunov, Scott R. Burt, M. Sabieh Anwar, Christian Hilty, Song-I. Han, Alexander Pines, and Renad Z. Sagdeev. para-Hydrogen-Induced Polarization in Heterogeneous Hydrogenation Reactions. Journal of the American Chemical Society, 129(17):5580-5586, 2007.

[92] Igor V. Koptyug, Anna A. Lysova, Renad Z. Sagdeev, and Valentin N. Parmon. Application of Multinuclear MRI and Solid State MRI in Heterogeneous Catalysis. Catalysis Today, 126(1-2):37-43, August 152007.

[93] Igor V. Koptyug, Dmitry R. Sagdeev, Edo Gerkema, Henk Van As, and Renad Z. Sagdeev. Solid-state 27Al MRI and NMR Thermometry for Catalytic Applications with Conventional (Liquids) MRI Instrumentation and Techniques. Journal of Magnetic Resonance, 175(1):21-29, July 2005. 
[94] Kirill V. Kovtunov, Irene E. Beck, Valery I. Bukhtiyarov, and Igor V. Koptyug. Observation of Parahydrogen-Induced Polarization in Heterogeneous Hydrogenation on Supported Metal Catalysts. Angewandte Chemie, International Edition, 47(8):14921495, February 82008.

[95] Dean O. Kuethe, Arvind Caprihan, H. Michael Gach, Irving J. Lowe, and Eiichi Fukushima. Imaging Obstructed Ventilation with NMR Using Inert Fluorinated Gases. Journal of Applied Physiology, 88(6):2279-2286, June 2000.

[96] Lars T. Kuhn and Joachim Bargon. Transfer of Parahydrogen-Induced Hyperpolarization to Heteronuclei. In situ NMR Methods in Catalysis, 276(n/a):25-68, 2007.

[97] M. Küppers, C. Heine, S. Han, Siegfried Stapf, and Bernhard Blümich. In Situ Observation of Diffusion and Reaction Dynamics in Gel Microreactors by Chemically Resolved NMR Microscopy. Applied Magnetic Resonance, 22(2):235, 2002.

[98] P. C. Lauterbur. Image Formation by Induced Local Interactions: Examples Employing Nuclear Magnetic Resonance. Nature, 242(5394):190, March 161973.

[99] S. Lee, M. Moessle, W. Myers, N. Kelso, A.H. Trabesinger, A. Pines, and J. Clarke. SQUID-Detected MRI at $132 \mu \mathrm{T}$ with $\mathrm{T} 1$ contrast weighted at $10 \mu \mathrm{T}-300 \mathrm{mT}$. Magnetic Resonance in Medicine, 53(1):9-14, 2005.

[100] Ira N. Levine. Molecular Spectroscopy. John Wiley \& Sons, 1975.

[101] Malcolm H. Levitt. Spin Dynamics: Basics of Nuclear Magnetic Resonance. John Wiley \& Sons, 1st edition, 2001. 
[102] Martin J. Lizak, Mark S. Conradi, and Charles G. Fry. NMR Imaging of Gas Imbibed into Porous Ceramic. Journal of Magnetic Resonance, 95(3):548-557, December 1991.

[103] Joaquin López-Serrano, Simon B. Duckett, Stuart Aiken, Karina Q. Almeida Almeida, Leñero, Eite Drent, John P. Dunne, Denis Konya, and Adrian C. Whitwood. A paraHydrogen Investigation of Palladium-Catalyzed Alkyne Hydrogenation. Journal of the American Chemical Society, 129(20):6513-6527, May 232007.

[104] Joaquin López-Serrano, Simon B. Duckett, and Agusti Lledós. Palladium-Catalyzed Hydrogenation: Detection of Palladium Hydrides. A Joint Study Using ParaHydrogen-Enhanced NMR Spectroscopy and Density Functional Theory. Journal of the American Chemical Society, 128(30):9596-9597, August 22006.

[105] M. W. Losey, R. J. Jackman, S. L. Firebaugh, M. A. Schmidt, and K. F. Jensen. Design and Fabrication of Microfluidic Devices for Multiphase Mixing and Reaction. Journal of Microelectromechanical Systems, 11(6):709-717, December 2002.

[106] Anna A. Lysova, Igor V. Koptyug, Alexander V. Kulikov, Valery A. Kirillov, Renad Z. Sagdeev, and Valentin N. Parmon. Nuclear Magnetic Resonance Imaging of an Operating Gas-liquid-solid Catalytic Fixed Bed Reactor. Chemical Engineering Journal, 130(2-3):101-109, June 12007.

[107] Anna A. Lysova, Igor V. Koptyug, Renad Z. Sagdeev, Valentin N. Parmon, Jaap A. Bergwerff, and Bert M. Weckhuysen. Noninvasive In Situ Visualization of Supported Catalyst Preparations Using Multinuclear Magnetic Resonance Imaging. Journal of the American Chemical Society, 127(34):11916-11917, August 312005. 
[108] J. R. MacFall, H. C. Charles, R. D. Black, H. Middleton, J. C. Swartz, B. Saam, B. Driehuys, C. Erickson, W. Happer, G. D. Cates, G. A. Johnson, and C. E. Ravin. Human Lung Air Spaces: Potential for MR Imaging with Hyperpolarized He-3. Radiology, 200:533-558, 1996.

[109] R. W. Mair, M. I. Hrovat, S. Patz, M. S. Rosen, I. C. Ruset, G. P. Topulos, L. L. Tsai, J. P. Butler, F. W. Hersman, and R. L. Walsworth. 3He Lung Imaging in an Open Access, Very-Low-Field Human Magnetic Resonance Imaging System. Magnetic Resonance in Medicine, 53(4):745-749, April 2005.

[110] Mitsuru Matsumoto and James H. Espenson. Kinetics of the Interconversion of Parahydrogen and Orthohydrogen Catalyzed by Paramagnetic Complex Ions. Journal of the American Chemical Society, 127(32):11447-11453, August 172005.

[111] Robert McDermott, SeungKyun Lee, Bennie ten Haken, Andreas H. Trabesinger, Alexander Pines, and John Clarke. Microtesla MRI with a Superconducting Quantum Interference Device. Proceedings of the National Academy of Sciences of the United States of America, 101(21):7857-7861, May 252004.

[112] Donald A. McQuarrie. Statistical Mechanics. University Science Books, Sausalito, California, 2000.

[113] Christian P. Mehnert, Edmund J. Mozeleski, and Raymond A. Cook. Supported Ionic Liquid Catalysis Investigated for Hydrogenation Reactions. Chemical Communications, 2002(24):3010-3011, 2002. 
[114] Christof Merckle, Simone Haubrich, and Janet Blümel. Immobilized Rhodium Hydrogenation Catalysts. Journal of Organometallic Chemistry, 627(1):44-54, May 4 2001.

[115] A. Messiah. Quantum Mechanics. Dover Publications, USA, 1958.

[116] Dieter Mewes, Thomas Loser, and Marco Millies. Modelling of Two-phase Flow in Packings and Monoliths. Chemical Engineering Science, 54(21):4729-4747, November 1999.

[117] Norbert Muller and Richard C. Reiter. Temperature Dependence of Chemical Shifts of Protons in Hydrogen Bonds. Journal of Chemical Physics, 42(9):3265-3269, 1965.

[118] Johannes Natterer and Joachim Bargon. Parahydrogen Induced Polarization. Progress in Nuclear Magnetic Resonance Spectroscopy, 31(4):293-315, November 1997.

[119] David Neuhaus and Michael P. Williamson. The Nuclear Overhauser Effect in Structural and Conformational Analysis. John Wiley \& Sons, 2nd edition, 2000.

[120] Heiko G. Niessen and Klaus Woelk. Investigations in Supercritical Fluids. In situ NMR Methods in Catalysis, 276(n/a):69-110, 2007.

[121] Dwight G. Nishimura. Principles of Magnetic Resonance Imaging. 1996.

[122] Dean L. Olson, Timothy L. Peck, Andrew G. Webb, Richard L Magin, and Jonathan V. Sweedler. High-Resolution Microcoil 1H-NMR for Mass-Limited, Nanoliter-Volume Samples. Science, 270:1967-1970, December 221995. 
[123] D. J. Parker and P. A. McNeil. Positron Emission Tomography for Process Applications. Measurement Science and Technology, 7(3):287-296, March 1996.

[124] Galina E. Pavlovskaya, Zackary I. Cleveland, Karl F. Stupic, Randall J. Basaraba, and Thomas Meersmann. Hyperpolarized Krypton-83 as a Contrast Agent for Magnetic Resonance Imaging. Proceedings of the National Academy of Sciences of the United States of America, 102(51):18275-18279, December 202005.

[125] A. Pines, M. G. Gibby, and J. S. Waugh. Proton-Enhanced Nuclear Induction Spectroscopy. A Method for High Resolution NMR of Dilute Spins in Solids. Journal of Chemical Physics, 56:1776-1777, 1972.

[126] C. J. Pouchert. The Aldrich Library of $13 C$ and 1 H FT-NMR Spectra. John Wiley \& Sons, 2008.

[127] Michael G. Pravica and Daniel P. Weitekamp. Net NMR Alignment by Adiabatic Transport of Parahydrogen Addition Products to High Magnetic Field. Chemical Physics Letters, 145(4):255-258, April 81988.

[128] E. M. Purcell, H. C. Torrey, and R. V. Pound. Physical Review, 69:37-38, 1946.

[129] W. T. Raynes, A. M. Davies, and D. B. Cook. Temperature-dependent chemical shifts and isotope shifts in the N.M.R. spectroscopy of hydrogen. Molecular Physics, 21(1):123-133, 1971.

[130] D. Christopher Roe, Peter M. Kating, Paul J. Krusic, and Bruce E. Smart. High 
Resolution NMR Techniques in Catalysis. Topics in Catalysis, 5(1-4):133-147, April 1998.

[131] Kai Ruppert, Jaime F. Mata, James R. Brookemand, Klaus D. Haspiel, and John P. III Mugler. Exploring Lung Function with Hyperpolarized 129Xe Nuclear Magnetic Resonance. Magnetic Resonance in Medicine, 51(4):676-687, April 2004.

[132] J. J. Sakurai. Modern Quantum Mechanics. Addison-Wesley, 1994.

[133] Andrew J. Sederman, Michael D. Mantle, Christopher P. Dunckley, Zhenyu Huang, and Lynn F. Gladden. In Situ MRI Study of 1-octene Isomerisation and Hydrogenation within a Trickle-bed Reactor. Catalysis Letters, 103(1-2):1-8, September 2005.

[134] Paul F. Seidler, Henry E. Bryndza, Jane E. Frommer, Louis S. Stuhl, and Robert G. Bergman. Synthesis of Trinuclear Alkylidyne Complexes from Dinuclear Alkyne Complexes and Metal Hydrides. CIDNP Evidence for Vinyl Radical Intermediates in the Hydrogenolysis of These Clusters. Organometallics, 2(11):1701-1705, November 1983.

[135] A. J. Shaka, C. J. Lee, and Alexander Pines. Iterative Schemes for Bilinear Operators: Applications to Spin Decoupling. Journal of Magnetic Resonance, 77:274-293, 1988.

[136] Shin-Guang Shyu, Sheau-Wen Cheng, and Der-Lii Tzou. Immobilization of $\mathrm{Rh}\left(\mathrm{PPh}_{3}\right)_{3} \mathrm{Cl}$ on Phosphinated MCM-41 for Catalytic Hydrogenation of Olefins. Chemical Communications, 1999(23):2337-2338, 1999.

[137] Chrétien Simons, Ulf Hanefeld, Isabel W. C. E. Arends, Adriaan J. Minnaard, Thomas 
Maschmeyer, and Roger A. Sheldon. Efficient Immobilisation of Rh-MonoPhos on the Aluminosilicate AlTUD-1. Chemical Communications, 2004(24):2830-2831, 2004.

[138] C. P. Slichter. Principles of Magnetic Resonance. Springer-Verlag, 3rd edition, 1992.

[139] Lars Sonderaaard, Freddy Stahlberg, Carsten Thomsen, Anders Stenggaard, Knud Lindvig, and Ole Henriksen. Accuracy and Precision of MR Velocity Mapping in Measurement of Stenotic Cross-sectional Area, Flow Rate, and Pressure Gradient. Journal of Magnetic Resonance Imaging, 3(2):433-437, 1993.

[140] O. W. Sørensen, G. W. Eich, Malcolm H. Levitt, G. Bodenhausen, and R. R. Ernst. Product Operator Formalism for the Description of NMR Pulse Experiments. Progress in Nuclear Magnetic Resonance Spectroscopy, 16:163-192, 1983.

[141] Joseph H Spur and Nuri Aksel. Fluid Mechanics. Springer, 2nd edition, 2008.

[142] S. Stapf and S.I. Han, editors. NMR Imaging in Chemical Engineering. John Wiley \& Sons, 2006.

[143] Walter A. Strauss. Partial Differential Equations: An Introduction. John Wiley \& Sons, 2nd edition, 2007.

[144] Davoud Tayebi, Hallvard F. Svendsen, Hugo A. Jakobsen, and Arne Grislingås. Measurement Techniques and Data Interpretations for Validating CFD Multi Phase Reactor Models. Chemical Engineering Communications, 186(1):56-159, 2001.

[145] Andreas Trabesinger, Robert McDermott, Michael Mueck, John Clarke, and Alexan- 
der Pines. SQUID Detected Liquid State NMR in Microtesla Fields. Journal of Physical Chemistry, 108:957-963, 2004.

[146] D. Traficante, editor. Well Logging, volume 13 of Concepts of Magnetic Resonance. John Wiley \& Sons, 2001.

[147] John Turkevich and Pierce W. Selwood. Solid Free Radical as Catalyst for Ortho-Para Hydrogen Conversion. Journal of the American Chemical Society, 63(4):1077-1079, 1941.

[148] J. Michael Tyszka, David H. Laidlaw, Joseph W. Asa, and Jeffrey M. Silverman. Three-Dimensional, Time-Resolved (4D) Relative Pressure Mapping Using Magnetic Resonance Imaging. Journal of Magnetic Resonance Imaging, 12:321-329, 2000.

[149] Stephen N. Urchuk and Donald B. Plewes. MR Measurement of Time-Dependent Blood Pressure Variations. Journal of Magnetic Resonance Imaging, 5:621-627, 1995.

[150] Ross D. Venook, Nathaniel I. Matter, Meena Ramachandran, Sharon E. Ungersma, Garry E. Gold, Nicholas J. Giori, Albert Macovski, Greig C. Scott, and Steven M. Conolly. Prepolarized MRI Around Metal Orthopedic Implants. Magnetic Resonance in Medicine, 56(1):177-186, 2006.

[151] Elena Vinogradov and Aaron K. Grant. Long-Lived States in Solution NMR: Selection Rules for Intramolecular Dipolar Relaxation in Low Magnetic Fields. Journal of Magnetic Resonance, 188(1):176-182, September 2007. 
[152] Paul Watts and Stephen J. Haswell. The Application of Microreactors for Small Scale Organic Synthesi. Chemical Engineering and Technology, 28(3):290-301, 2005.

[153] George M. Whitesides. The Origins and the Future of Microfluidics. Nature, 442(7101):368-373, July 272006.

[154] Andrew M. Wolters, Dimuthu A. Jayawickrama, and Jonathan V. Sweedler. Microscale NMR. Current Opinion in Chemical Biology, 6(5):711-716, October 12002.

[155] Nicholas J. Wood, James A. Brannigan, Simon B. Duckett, Sarah L. Heath, and Jane Wagstaff. Detection of Picomole Amounts of Biological Substrates by para-HydrogenEnhanced NMR Methods in Conjunction with a Suitable Receptor Complex. Journal of the American Chemical Society, 129(36):11012-11013, September 122007.

[156] Shoujun Xu, Valeriy V. Yashchuk, Marcus H. Donaldson, Simon M. Rochester, Dmitry Budker, and Alexander Pines. Magnetic resonance imaging with an optical atomic magnetometer. Proceedings of the National Academy of Sciences, 103:12668-12671, 2006.

[157] Shoujun Xu, Valeriy V. Yashchuk, Marcus H. Donaldson, Simon M. Rochester, Dmitry Budker, and Alexander Pines. Magnetic Resonance Imaging with an Optical Atomic Magnetometer. Proceedings of the National Academy of Sciences of the United States of America, 103(34):12668-12671, August 222006.

[158] Chekmenev: Eduard Y., Jan Hövener, Valerie A. Norton, Kent Harris, Lynne S. Batchelder, Pratip Bhattacharya, Brian D. Ross, and Daniel P. Weitekamp. PASA- 
DENA Hyperpolarization of Succinic Acid for MRI and NMR Spectroscopy. Journal of the American Chemical Society, 130(13):4212-4213, April 22008.

[159] Guang-Zhong Yang, Philip J. Kilner, Nigel B. Wood, S. Richard Underwood, and David N. Firmin. Computation of flow pressure fields from magnetic resonance velocity mapping. Magnetic Resonance in Medicine, 36:520-526, 1996.

[160] E. H. L. Yuen, Andrew J. Sederman, and Lynn F. Gladden. In situ Magnetic Resonance Visualisation of the Spatial Variation of Catalytic Conversion Within a Fixedbed Reactor. Applied Catalysis A: General, 232(1-2):29-38, June 102002.

[161] Rongrong Zhou, Juan A. Aguilar, Adrian Charlton, Simon B. Duckett, Paul I. P. Elliott, and Rathika Kandiah. Parahydrogen Derived Illumination of Pyridine Based Coordination Products Obtained from Reactions Involving Rhodium Phosphine Complexes. Dalton Transactions, 2005(23):3773-3779, 2005. 